\title{
Unified Total Synthesis of Five Bufadienolides
}

\author{
Shinsuke Shimizu, Koichi Hagiwara, Hiroaki Itoh, and Masayuki Inoue* \\ Graduate School of Pharmaceutical Sciences \\ The University of Tokyo, Hongo, Bunkyo-ku, Tokyo 113-0033, Japan \\ Fax: (+81)3-5841-0568 \\ E-mail:inoue@mol.f.u-tokyo.ac.jp
}

Supporting Information

77 pages

Contents:

1. Experimental procedures

2. Comparison of ${ }^{1} \mathrm{H}$ and ${ }^{13} \mathrm{C}\left\{{ }^{1} \mathrm{H}\right\}$ NMR data of the reported and synthetic 1-5 $\quad$ S27

3. X-ray structures and crystallographic data of $\mathbf{6}$ and $\mathbf{1 8}$

4. Biological experiments

S42

5. References

S45

6. NMR charts

S46 


\section{Experimental procedures}

General method: All reactions sensitive to air or moisture were carried out under argon atmosphere in dry solvents unless otherwise noted. $\mathrm{CH}_{2} \mathrm{Cl}_{2}$, DMF, THF, and toluene were purified by Glass Contour solvent dispensing system (Nikko Hansen \& Co., Ltd.). All other reagents were used as supplied. All reactions under heating conditions were performed in an oil bath. Analytical thin-layer chromatography (TLC) was performed using E. Merck Silica gel $60 \mathrm{~F}_{254}$ pre-coated plates $(0.25 \mathrm{~mm})$. Flash column chromatography was performed using 40-50 $\mu \mathrm{m}$ Silica Gel 60N (Kanto Chemical Co., Inc.), 40-63 $\mu \mathrm{m}$ Silica Gel 60 (Merck), or 32$53 \mu \mathrm{m}$ Silica Gel BW-300 (Fuji Silysia Chemical Ltd.). Preparative thin-layer chromatography (PTLC) was performed using Merck silica gel $60 \mathrm{~F}_{254}$ pre-coated plates $(0.5$ $\mathrm{mm}$ ). Preparative gel permeation chromatography (GPC) was performed on LaboACE LC5060 (Japan Analytical Industry Co., Ltd.) equipped with JAIGEL-2HR column using UV (254 $\mathrm{nm}$ ) and Refractive Index (RI) detectors in $\mathrm{CHCl}_{3}$ with flow rate of $10.0 \mathrm{~mL} / \mathrm{min}$. High performance liquid chromatography (HPLC) was performed on a JASCO HPLC system (pump: JASCO PU-2086 Plus x2, detector: JASCO MD-4010, degasser: ERC Inc. ERC-3325, data analysis by JASCO ChromNAV 1.5.2.). Melting points were measured on Yanaco MP-J3 micro melting point apparatus and were uncorrected. Optical rotations were measured on JASCO P-2200 polarimeter at ambient temperature using the sodium D line. Infrared (IR) spectra were recorded on JASCO FT/IR-4100 spectrometer as a thin film on $\mathrm{KBr}$ or $\mathrm{CaF}_{2} .{ }^{1} \mathrm{H}$ and ${ }^{13} \mathrm{C}\left\{{ }^{1} \mathrm{H}\right\}$ NMR spectra were recorded on JEOL JNM-ECX-500, JNM-ECZ-500, or JNMECS-400 spectrometer. Chemical shifts were denoted in ppm on the $\delta$ scale relative to residual solvent peaks as internal standard: $\mathrm{CHCl}_{3}\left(\delta 7.26\right.$ for $\left.{ }^{1} \mathrm{H} \mathrm{NMR}\right), \mathrm{CDCl}_{3}(\delta 77.0$ for $\left.{ }^{13} \mathrm{C}\left\{{ }^{1} \mathrm{H}\right\} \mathrm{NMR}\right), \mathrm{C}_{6} \mathrm{D}_{5} \mathrm{H}\left(\delta 7.16\right.$ for ${ }^{1} \mathrm{H}$ NMR), $\mathrm{C}_{6} \mathrm{D}_{6}\left(\delta 128.0\right.$ for $\left.{ }^{13} \mathrm{C}\left\{{ }^{1} \mathrm{H}\right\} \mathrm{NMR}\right), \mathrm{CD}_{2} \mathrm{HOD}(\delta$ 3.31 for $\left.{ }^{1} \mathrm{H} N M R\right), \mathrm{CD}_{3} \mathrm{OD}\left(\delta 49.0\right.$ for $\left.{ }^{13} \mathrm{C}\left\{{ }^{1} \mathrm{H}\right\} \mathrm{NMR}\right), \mathrm{D}_{3} \mathrm{CSOCD}_{2} \mathrm{H}\left(\delta 2.50\right.$ for $\left.{ }^{1} \mathrm{H} \mathrm{NMR}\right)$, and $\mathrm{D}_{3} \mathrm{CSOCD}_{3}\left(\delta 39.52\right.$ for ${ }^{13} \mathrm{C}\left\{{ }^{1} \mathrm{H}\right\}$ NMR). Signal patterns are indicated as s, singlet; $d$, doublet; $\mathrm{t}$, triplet; $\mathrm{m}$, multiplet; br, broaden peak. The carbon numbering of compounds corresponds to that of vulgarobufotoxin (4) and 3-( $N$-succinyl argininyl) bufotalin (5), unless otherwise noted. High resolution mass spectra were measured on JEOL JMS-T100LP (ESITOF) or BRUKER DALTONICS microTOF II (ESI-TOF). X-ray crystallographic analysis was conducted using Inorganic Fine Crystal Structural Diffractometer VariMax Dual (Rigaku Co., Ltd.) with Mo radiation $(\lambda=0.71073 \AA)$. 

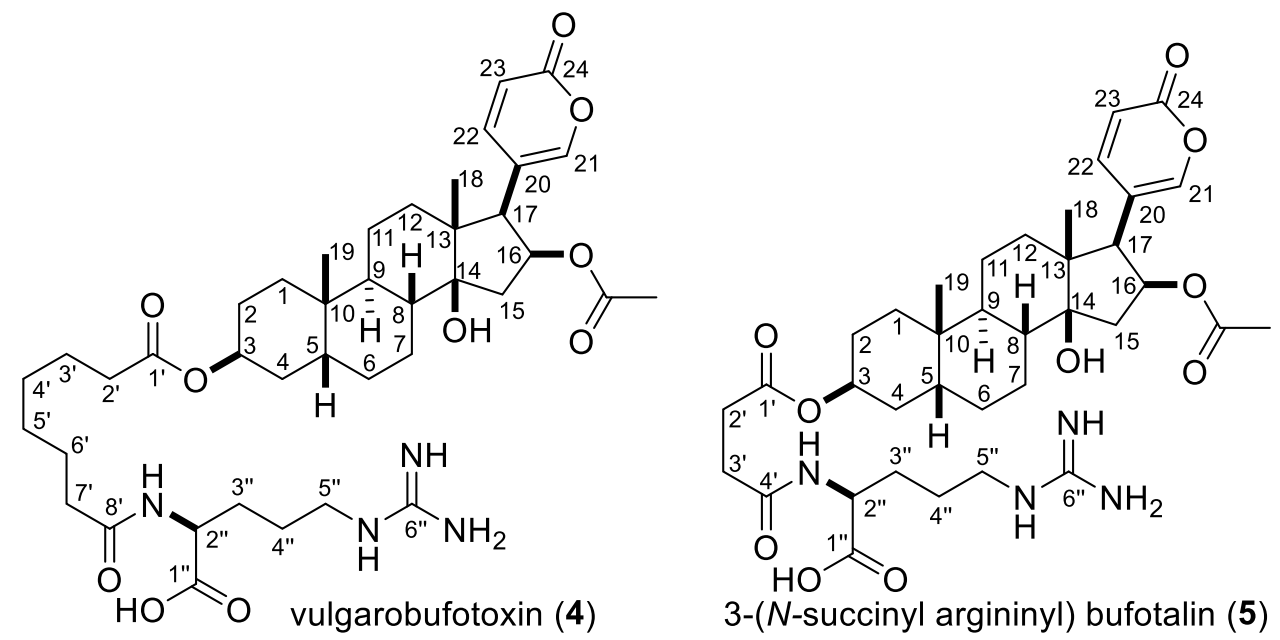

Figure S1. Numbering system of vulgarobufotoxin (4) and 3-( $N$-succinyl argininyl) bufotalin (5)<smiles>CC12CCC3C(CCC4CC(=O)CCC43C)C1CCC2=O</smiles>

9

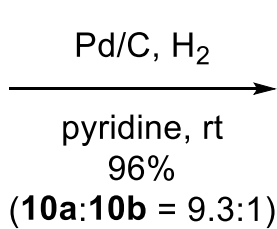

$(10 \mathrm{a}: 10 \mathrm{~b}=9.3: 1)$

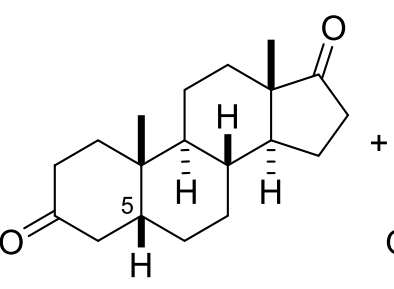

$10 \mathrm{a}$<smiles>CC12CC[C@H]3[C@H](CC[C@@H]4CC(=O)CC[C@]34C)[C@@H]1CCC2=O</smiles>

$10 \mathrm{~b}$

Diketones 10a [CAS: 1229-12-5] ${ }^{\mathrm{S} 1}$ and 10b [CAS: 846-46-8]. ${ }^{\mathrm{S} 2} \quad$ A suspension of $\mathrm{Pd} / \mathrm{C}(10$ $\mathrm{wt} \% \mathrm{Pd}$ on carbon, $499 \mathrm{mg})$ in pyridine $(15 \mathrm{~mL})$ was stirred at room temperature for $30 \mathrm{~min}$. Compound 9 (5.53 g, $19.3 \mathrm{mmol})$ was added to the suspension, and the resultant suspension was stirred under $\mathrm{H}_{2}$ atmosphere $(1 \mathrm{~atm})$ at room temperature for $12 \mathrm{~h}$. The reaction mixture was then filtered through a pad of Celite with $\mathrm{CH}_{2} \mathrm{Cl}_{2}(15 \mathrm{~mL})$ and concentrated. After $1 \mathrm{M}$ aqueous $\mathrm{HCl}(15 \mathrm{~mL}, 15 \mathrm{mmol})$ was added to the residue, the resultant mixture was extracted with $\mathrm{CH}_{2} \mathrm{Cl}_{2}$ (30 mL x6). The combined organic layers were dried over $\mathrm{Na}_{2} \mathrm{SO}_{4}$, filtered, and concentrated. The residue was purified by flash column chromatography on silica gel (100 g, hexane $/ \mathrm{CH}_{2} \mathrm{Cl}_{2}=3 / 1$ to $0 / 1$ ) to afford a 9.3:1 diastereomeric mixture of diketones 10a and 10b $(5.34 \mathrm{~g}, 18.5 \mathrm{mmol})$ in $96 \%$ yield. The $\mathrm{C} 5$-stereochemistry of $\mathbf{1 0 a}$ was determined by the Xray crystallographic analysis of epoxide 6: white solid. m.p. $121-123{ }^{\circ} \mathrm{C}$. $[\alpha] \mathrm{D}^{27}+98(c 0.96$, $\mathrm{CHCl}_{3}$ ). IR (film) 2934, 2861, 1738, 1712, 1452, 1377, 1266, 1248, $1051 \mathrm{~cm}^{-1} .{ }^{1} \mathrm{H}$ NMR (500 $\left.\mathrm{MHz}, \mathrm{CD}_{3} \mathrm{OD}\right)$ peaks of the major product 10a: $\delta 0.89(3 \mathrm{H}, \mathrm{s}, \mathrm{H} 18), 1.07(3 \mathrm{H}, \mathrm{s}, \mathrm{H} 19), 1.30-$ $1.80(13 \mathrm{H}, \mathrm{m}), 1.95-2.10(6 \mathrm{H}, \mathrm{m}), 2.41-2.48(2 \mathrm{H}, \mathrm{m}), 2.84(1 \mathrm{H}, \mathrm{dd}, J=14.6,14.6 \mathrm{~Hz}) .{ }^{13} \mathrm{C}\left\{{ }^{1} \mathrm{H}\right\}$ $\mathrm{NMR}\left(125 \mathrm{MHz}, \mathrm{CD}_{3} \mathrm{OD}\right)$ peaks of the major product 10a: $\delta 14.2,21.5,22.7,22.9,25.7,27.5$, 32.8, 36.2, 36.4, 36.7, 37.9, 38.1, 41.8, 43.1, 45.8, 49.0 (deduced from HMBC), 52.4, 215.6, 223.5. HRMS (ESI-TOF) $[\mathrm{M}+\mathrm{Na}]^{+} \mathrm{m} / \mathrm{z}$ : Calcd for $\mathrm{C}_{19} \mathrm{H}_{28} \mathrm{O}_{2} \mathrm{Na} 311.1987$; Found 311.1976. 

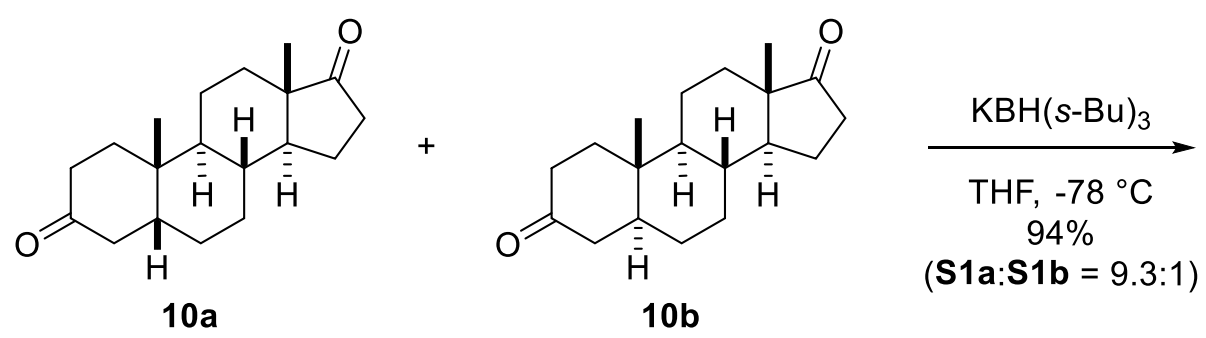

$(10 a: 10 b=9.3: 1)$

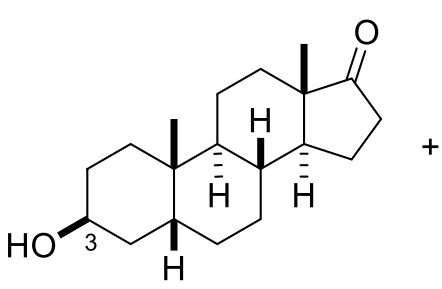

S1a<smiles>CC12CC[C@H]3[C@@H](CC[C@H]4CC(O)CCC43C)[C@@H]1CCC2=O</smiles>

S1b

Ketones S1a [CAS: 571-31-3] ${ }^{\mathrm{S} 3}$ and S1b. $\mathrm{KBH}(s-\mathrm{Bu})_{3}(1.0 \mathrm{M}$ THF solution, $18.5 \mathrm{~mL}, 18.5$ mmol) was added to a solution of a 9.3:1 diastereomeric mixture of diketones 10a and 10b (5.34 $\mathrm{g}, 18.5 \mathrm{mmol})$ in THF $(185 \mathrm{~mL})$ at $-78{ }^{\circ} \mathrm{C}$. After the reaction mixture was stirred at $-78{ }^{\circ} \mathrm{C}$ for $5.5 \mathrm{~h}, 2.6 \mathrm{M}$ aqueous $\mathrm{NaOH}(7.0 \mathrm{~mL}, 18 \mathrm{mmol})$ and aqueous $\mathrm{H}_{2} \mathrm{O}_{2}(30 \mathrm{wt} \%, 9.8 \mathrm{M}, 7.0 \mathrm{~mL}$, $69 \mathrm{mmol}$ ) were added to the mixture. The resultant mixture was concentrated and extracted with EtOAc $(50 \mathrm{~mL} \times 5)$. The combined organic layers were dried over $\mathrm{Na}_{2} \mathrm{SO}_{4}$, filtered, and concentrated. The residue was purified by flash column chromatography on silica gel $(150 \mathrm{~g}$, hexane/EtOAc $=1 / 0$ to 20/1 to 10/1) to afford a 9.3:1 diastereomeric mixture of ketones S1a and S1b (5.06 g, $17.4 \mathrm{mmol}$ ) in 94\% yield. The C3-stereochemistry of S1a was determined by the X-ray crystallographic analysis of epoxide 6: white solid. m.p. $163-166{ }^{\circ} \mathrm{C}$. $[\alpha]_{\mathrm{D}}{ }^{27}+62$ (c 1.10, $\mathrm{CHCl}_{3}$ ). IR (film) 3218, 2929, 2859, 1738, 1451, 1405, 1324, 1197, $1031 \mathrm{~cm}^{-1} .{ }^{1} \mathrm{H}$ NMR $\left(500 \mathrm{MHz}, \mathrm{CDCl}_{3}\right)$ peaks of the major product S1a: $\delta 0.85(3 \mathrm{H}, \mathrm{s}, \mathrm{H} 18), 0.98(3 \mathrm{H}, \mathrm{s}$, H19), 1.07-1.17 (1H, m), 1.19-1.42 (7H, m), 1.44-1.66 (7H, m), 1.73-1.82 (2H, m), 1.88-1.99 $(3 \mathrm{H}, \mathrm{m}), 2.06(1 \mathrm{H}, \mathrm{ddd}, J=19.2,9.4,9.4 \mathrm{~Hz}), 2.43(1 \mathrm{H}, \mathrm{dd}, J=19.2,8.9 \mathrm{~Hz}), 4.11(1 \mathrm{H}, \mathrm{dd}, J$ $=2.8,2.8 \mathrm{~Hz}, \mathrm{H} 3) .{ }^{13} \mathrm{C}\left\{{ }^{1} \mathrm{H}\right\} \mathrm{NMR}\left(125 \mathrm{MHz}, \mathrm{CDCl}_{3}\right)$ peaks of the major product S1a: $\delta 13.8$, 20.3, 21.8, 23.8, 25.2, 26.3, 27.8, 29.9, 31.7, 33.4, 35.2, 35.3, 35.9, 36.5, 40.0, 47.9, 51.6, 66.9, 221.5. HRMS (ESI-TOF) $[\mathrm{M}+\mathrm{Na}]^{+} \mathrm{m} / \mathrm{z}$ : Calcd for $\mathrm{C}_{19} \mathrm{H}_{30} \mathrm{O}_{2} \mathrm{Na} 313.2143$; Found 313.2140.

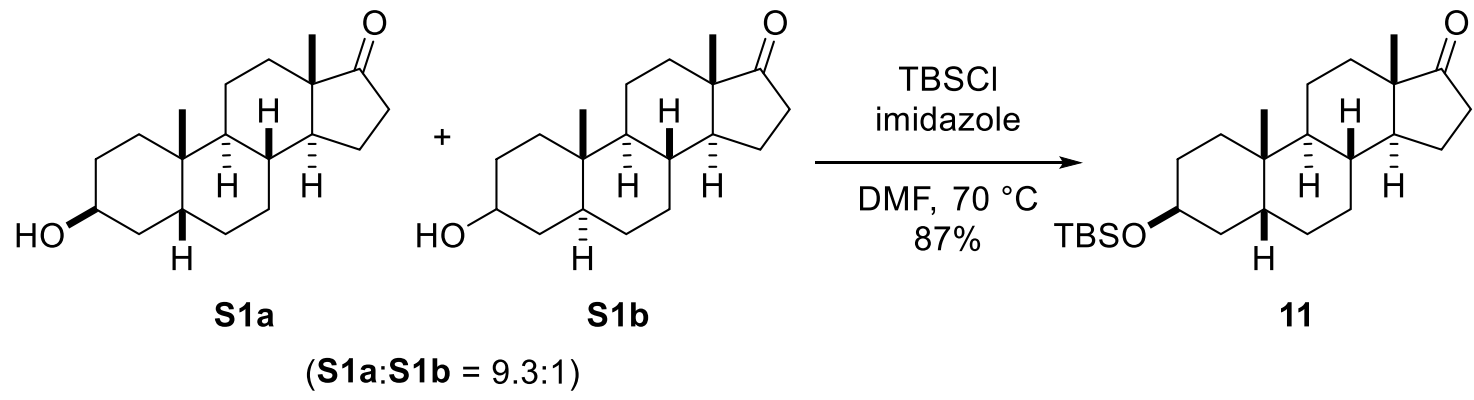

Ketone 11 [CAS: 65598-74-5]. ${ }^{\mathrm{S} 4} \quad t$-Butyldimethylsilyl chloride (TBSCl, 15.7 g, 104 mmol) was added to a solution of a 9.3:1 diastereomeric mixture of ketones S1a and S1b (15.1 g, 52.0 
mmol), and imidazole $(14.1 \mathrm{~g}, 207 \mathrm{mmol})$ in DMF $(73 \mathrm{~mL})$ at room temperature. After the reaction mixture was stirred at $70{ }^{\circ} \mathrm{C}$ for $15 \mathrm{~h}$, saturated aqueous $\mathrm{NaHCO}_{3}(100 \mathrm{~mL})$ and $\mathrm{H}_{2} \mathrm{O}$ $(50 \mathrm{~mL})$ were added to the mixture at $0{ }^{\circ} \mathrm{C}$. The resultant mixture was extracted with $\mathrm{Et}_{2} \mathrm{O}$ $(100 \mathrm{~mL} x 4)$. The combined organic layers were washed with brine $(30 \mathrm{~mL})$, dried over $\mathrm{Na}_{2} \mathrm{SO}_{4}$, filtered, and concentrated. The residue was purified by flash column chromatography on silica gel $(250 \mathrm{~g}$, hexane/Et $2 \mathrm{O}=100 / 1$ then hexane/EtOAc $=3 / 1)$ to afford ketone 11 (18.2 g, $45.0 \mathrm{mmol})$ in $87 \%$ yield: white solid. m.p. $121-123{ }^{\circ} \mathrm{C}$. $[\alpha]_{\mathrm{D}}{ }^{27}+70(c 0.47$, $\mathrm{CHCl}_{3}$ ). IR (film) 2927, 2857, 1742, 1449, 1406, 1373, 1253, 1163, 1057, $1005 \mathrm{~cm}^{-1} .{ }^{1} \mathrm{H}$ NMR (400 MHz, C6 6 6) $\delta 0.10$ (6H, s, CH3 of TBS), 0.62 (3H, s, H18), 0.88 (3H, s, H19), 0.94-1.41 $(13 \mathrm{H}, \mathrm{m}), 1.02(9 \mathrm{H}, \mathrm{s}, t$-Bu of TBS $), 1.47-1.56(2 \mathrm{H}, \mathrm{m}), 1.61-1.96(6 \mathrm{H}, \mathrm{m}), 2.16(1 \mathrm{H}, \mathrm{dd}, J=$ 19.2, 9.2 Hz, H16a), 4.02 (1H, brs, H3). ${ }^{13} \mathrm{C}\left\{{ }^{1} \mathrm{H}\right\}$ NMR (125 MHz, $\left.\mathrm{C}_{6} \mathrm{D}_{6}\right) \delta-4.66,13.7,18.4$, 20.6, 21.8, 24.0, 25.5, 26.1, 26.9, 28.8, 30.4, 32.4, 34.7, 35.3, 35.4, 35.7, 37.0, 40.4, 47.6, 51.4, 67.9, 218.2. HRMS (ESI-TOF) $[\mathrm{M}+\mathrm{Na}]^{+} \mathrm{m} / \mathrm{z}$ : Calcd for $\mathrm{C}_{25} \mathrm{H}_{44} \mathrm{O}_{2} \mathrm{SiNa}$ 427.3008; Found 427.3014 .

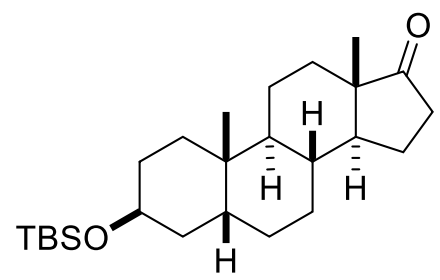

11

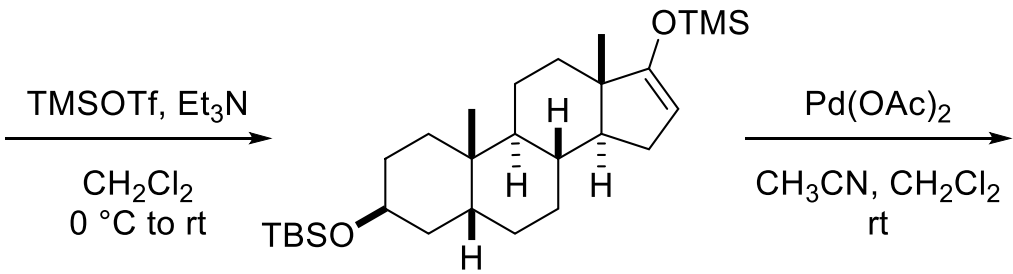

S2

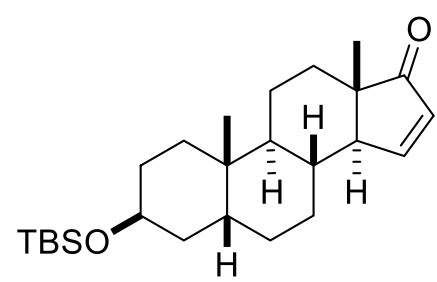

12

$73 \%$ (2 steps)

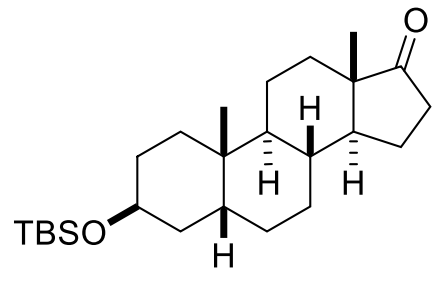

11

$15 \%$ (2 steps)

Enone 12 [CAS: 64647-72-9]. ${ }^{\mathrm{S} 4}$ Trimethylsilyl trifluoromethanesulfonate (TMSOTf, $24 \mathrm{~mL}$, $0.13 \mathrm{~mol}$ ) was added to a solution of ketone $11(18.2 \mathrm{~g}, 45.0 \mathrm{mmol})$ and $\mathrm{Et}_{3} \mathrm{~N}(25 \mathrm{~mL}, 0.18 \mathrm{~mol})$ in $\mathrm{CH}_{2} \mathrm{Cl}_{2}(900 \mathrm{~mL})$ at $0{ }^{\circ} \mathrm{C}$. After the reaction mixture was stirred at $0{ }^{\circ} \mathrm{C}$ for $20 \mathrm{~min}$ and at room temperature for $40 \mathrm{~min}$, saturated aqueous $\mathrm{NaHCO}_{3}(300 \mathrm{~mL})$ was added to the mixture. The resultant mixture was extracted with hexane $(600 \mathrm{~mL} \times 3)$. The combined organic layers were washed with brine ( $100 \mathrm{~mL} \times 2)$, dried over $\mathrm{Na}_{2} \mathrm{SO}_{4}$, filtered, and concentrated to afford the crude silyl enol ether S2 [CAS: $914644-97-6]^{\mathrm{S} 4}$ (19.9 g), which was used in the next reaction without further purification.

$\mathrm{Pd}(\mathrm{OAc})_{2}(12.1 \mathrm{~g}, 53.9 \mathrm{mmol})$ was added to a solution of the above crude silyl enol ether $\mathbf{S 2}$ (19.9 g) in $\mathrm{CH}_{3} \mathrm{CN}(720 \mathrm{~mL})$ and $\mathrm{CH}_{2} \mathrm{Cl}_{2}(180 \mathrm{~mL})$ at room temperature. After the reaction mixture was stirred at room temperature for $25 \mathrm{~h}$, saturated aqueous $\mathrm{NaHCO}_{3}(300 \mathrm{~mL})$ was added to the mixture. The resultant mixture was filtered through a pad of Celite with EtOAc 
$(300 \mathrm{~mL})$ and extracted with EtOAc $(300 \mathrm{~mL} x 3)$. The combined organic layers were washed with brine $(200 \mathrm{~mL})$, dried over $\mathrm{Na}_{2} \mathrm{SO}_{4}$, filtered, and concentrated. The residue was purified by flash column chromatography on silica gel $(500 \mathrm{~g}$, hexane/EtOAc $=100 / 1$ to $20 / 1)$ to afford enone 12 (13.2 g, $32.8 \mathrm{mmol})$ and ketone 11 (2.66 g, $6.57 \mathrm{mmol})$ in 73\% and 15\% yields over 2 steps, respectively: white solid. m.p. $122-124{ }^{\circ} \mathrm{C}$. $[\alpha]_{\mathrm{D}}{ }^{25}-17\left(c 1.74, \mathrm{CHCl}_{3}\right)$. IR (film) 2931, 2858, 1711, 1455, 1369, 1252, 1058, $1003 \mathrm{~cm}^{-1} .{ }^{1} \mathrm{H}$ NMR $\left(400 \mathrm{MHz}, \mathrm{CDCl}_{3}\right) \delta 0.01(6 \mathrm{H}, \mathrm{s}$, $\mathrm{CH}_{3}$ of TBS), 0.88 (9H, s, $t$-Bu of TBS), $1.00(3 \mathrm{H}, \mathrm{s}), 1.04(3 \mathrm{H}, \mathrm{s}), 1.16-1.27(3 \mathrm{H}, \mathrm{m}), 1.38-$ $1.58(8 \mathrm{H}, \mathrm{m}), 1.70-1.74(1 \mathrm{H}, \mathrm{m}), 1.81-1.99(5 \mathrm{H}, \mathrm{m}), 2.33(1 \mathrm{H}, \mathrm{d}, J=11.2 \mathrm{~Hz}), 4.03(1 \mathrm{H}, \mathrm{s}, \mathrm{H} 3)$, $6.01(1 \mathrm{H}, \mathrm{dd}, J=5.5,3.0 \mathrm{~Hz}, \mathrm{H} 16), 7.52(1 \mathrm{H}, \mathrm{d}, J=5.5 \mathrm{~Hz}, \mathrm{H} 15) .{ }^{13} \mathrm{C}\left\{{ }^{1} \mathrm{H}\right\} \mathrm{NMR}(100 \mathrm{MHz}$, $\left.\mathrm{CDCl}_{3}\right) \delta-4.88,-4.86,18.1,20.0,20.7,23.9,25.4,25.8,26.5,28.5,29.4,29.9,32.7,34.4,35.4$, 36.5, 41.6, 51.2, 57.2, 67.1, 131.6, 158.8, 213.6. HRMS (ESI-TOF) $[\mathrm{M}+\mathrm{Na}]^{+} \mathrm{m} / \mathrm{z}$ : Calcd for $\mathrm{C}_{25} \mathrm{H}_{42} \mathrm{O}_{2} \mathrm{SiNa}$ 425.2852; Found 425.2849.

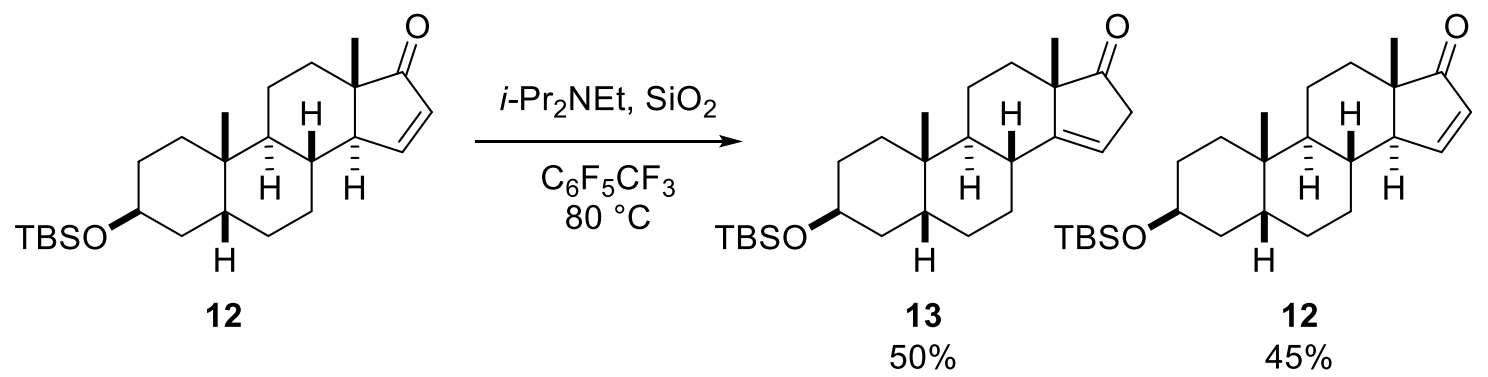

Ketone 13 [CAS: 914644-98-7]. ${ }^{\mathrm{S} 4} \quad i$ - $\mathrm{Pr}_{2} \mathrm{NEt}(160 \mathrm{~mL}, 918 \mathrm{mmol})$ was added to a suspension of enone 12 (13.5 g, $33.5 \mathrm{mmol})$ and $\mathrm{SiO}_{2}(50.3 \mathrm{~g})$ in $\mathrm{C}_{6} \mathrm{~F}_{5} \mathrm{CF}_{3}(85 \mathrm{~mL})$ at room temperature. After being stirred at $80{ }^{\circ} \mathrm{C}$ for $4 \mathrm{~h}$, the reaction mixture was filtered through a pad of Celite with EtOAc $(500 \mathrm{~mL})$ and concentrated. The residue was purified by flash column chromatography on silica gel ( $500 \mathrm{~g}$, hexane/EtOAc $=1 / 0$ to $100 / 1$ to $20 / 1)$ to afford ketone $\mathbf{1 3}$ $(6.81 \mathrm{~g}, 16.9 \mathrm{mmol})$ and enone $12(6.13 \mathrm{~g}, 15.2 \mathrm{mmol})$ in $50 \%$ and $45 \%$ yields, respectively: colorless oil. $[\alpha]_{\mathrm{D}}{ }^{26}+111\left(c 0.38, \mathrm{CHCl}_{3}\right)$. IR (film) 2930, 2860, 1747, 1639, 1449, 1376, 1254 , 1158, 1057, $1009 \mathrm{~cm}^{-1} .{ }^{1} \mathrm{H}$ NMR (400 MHz, $\left.\mathrm{CDCl}_{3}\right) \delta 0.00(6 \mathrm{H}, \mathrm{s}, \mathrm{CH} 3$ of TBS), $0.87(9 \mathrm{H}, \mathrm{s}$, $t$-Bu of TBS), $0.98(3 \mathrm{H}, \mathrm{s}), 1.09(3 \mathrm{H}, \mathrm{s}), 1.20-1.26(3 \mathrm{H}, \mathrm{m}), 1.32-1.43(5 \mathrm{H}, \mathrm{m}), 1.48-1.55(2 \mathrm{H}$, $\mathrm{m}), 1.56-1.61(2 \mathrm{H}, \mathrm{m}), 1.74-1.82(3 \mathrm{H}, \mathrm{m}), 1.91-1.99(1 \mathrm{H}, \mathrm{m}), 2.17(1 \mathrm{H}, \mathrm{brs}), 2.79-2.85(1 \mathrm{H}, \mathrm{m}$, H16a), 2.95-3.02 (1H, m, H16b), $4.00(1 \mathrm{H}, \mathrm{s}, \mathrm{H} 3), 5.47(1 \mathrm{H}, \mathrm{d}, J=1.6 \mathrm{~Hz}, \mathrm{H} 15) .{ }^{13} \mathrm{C}\left\{{ }^{1} \mathrm{H}\right\} \mathrm{NMR}$ $\left(100 \mathrm{MHz}, \mathrm{CDCl}_{3}\right) \delta-4.90,-4.87,18.1,19.9,20.8,23.1,23.7,25.8,26.3,28.6,29.7,33.5,34.2$, 35.5, 35.6, 36.4, 41.1, 41.4, 51.0, 67.2, 112.6, 154.1, 222.9. HRMS (ESI-TOF) $[\mathrm{M}+\mathrm{Na}]^{+} \mathrm{m} / \mathrm{z}$ : Calcd for $\mathrm{C}_{25} \mathrm{H}_{42} \mathrm{O}_{2} \mathrm{SiNa} 425.2852$; Found 425.2835. 


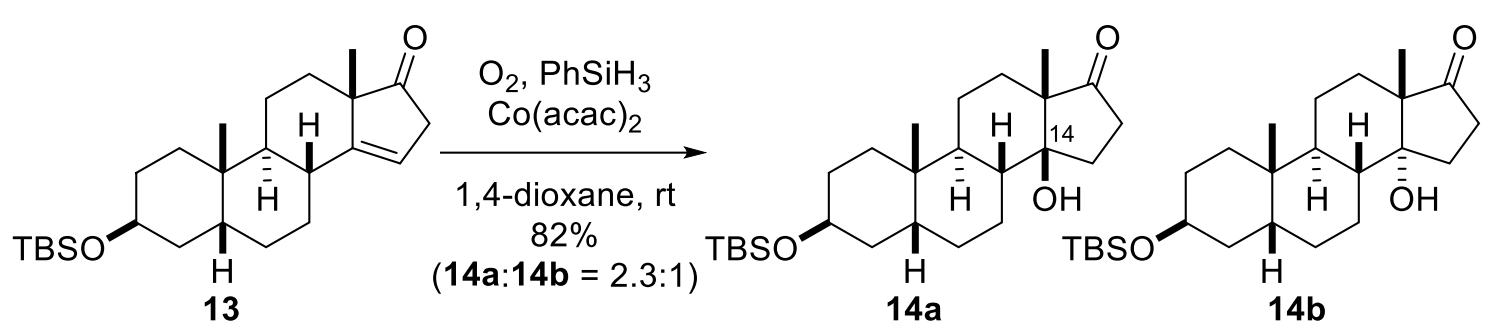

Ketones 14a and 14b. A solution of ketone 13 (10.1 g, $25.1 \mathrm{mmol})$ in 1,4-dioxane (450 mL) was bubbled with $\mathrm{O}_{2}$ for $25 \mathrm{~min}$ at room temperature. Then cobalt(II) acetylacetonate (Co(acac)2, $1.30 \mathrm{~g}, 5.06 \mathrm{mmol}$ ) was added to the mixture at room temperature. A solution of $\mathrm{PhSiH}_{3}(9.3 \mathrm{~mL}, 75 \mathrm{mmol})$ in 1,4-dioxane $(50 \mathrm{~mL})$ was added to the mixture at room temperature over $1 \mathrm{~h}$ via a syringe pump under $\mathrm{O}_{2}$ atmosphere $(1 \mathrm{~atm})$. After the reaction mixture was stirred under $\mathrm{O}_{2}$ atmosphere ( $1 \mathrm{~atm}$ ) at room temperature for $4 \mathrm{~h}$, saturated aqueous $\mathrm{Na}_{2} \mathrm{~S}_{2} \mathrm{O}_{3}(200 \mathrm{~mL})$ was added to the mixture. The resultant mixture was extracted with EtOAc $(200 \mathrm{~mL} \mathrm{x} 4)$. The combined organic layers were washed with brine $(50 \mathrm{~mL})$, dried over $\mathrm{Na}_{2} \mathrm{SO}_{4}$, filtered, and concentrated. The residue was purified by flash column chromatography on silica gel $(500 \mathrm{~g}$, hexane/EtOAc $=10 / 1$ to $3 / 1)$ to afford a 2.3:1 mixture of ketones $14 \mathbf{a}$ and $\mathbf{1 4 b}(8.71 \mathrm{~g}, 20.7 \mathrm{mmol})$ in $82 \%$ yield. The C14-stereochemistry of $\mathbf{1 4 a}$ was determined by the X-ray crystallographic analysis of epoxide 6: white solid. m.p. 148-149 ${ }^{\circ} \mathrm{C}$. $[\alpha]_{\mathrm{D}}{ }^{25}+21\left(c 1.65, \mathrm{CHCl}_{3}\right)$. IR (film) 3426, 2931, 2888, 2858, 1732, 1469, 1447, 1376, 1361, 1253, $1064 \mathrm{~cm}^{-1} .{ }^{1} \mathrm{H}$ NMR (400 MHz, $\left.\mathrm{CDCl}_{3}\right) \delta 0.01\left(6 \mathrm{H}, \mathrm{s}, \mathrm{CH}_{3}\right.$ of TBS), 0.87 (9H, s, $t$-Bu of TBS), 0.93 (3H x7/10, s, H19), 0.96 (1H x3/10, s, H19), 0.97 (3H x3/10, s, H18), 1.04 (3H $\mathrm{x} 7 / 10, \mathrm{~s}, \mathrm{H} 18), 1.10-1.91(18 \mathrm{H}$ and $1 \mathrm{H} \mathrm{x} 3 / 10, \mathrm{~m}), 2.13-2.21$ (1H x7/10, m, H15a), 2.33-2.47 (2H, m, H16), 3.94-4.09 (1H, m, H3). ${ }^{13} \mathrm{C}\left\{{ }^{1} \mathrm{H}\right\}$ NMR (100 MHz, $\left.\mathrm{CDCl}_{3}\right) \delta-4.9,12.8(1 \mathrm{C} \times 7 / 10)$, 18.0 (1C x3/10), 18.1 (1C x7/10), 19.1 (1C x3/10), 19.6 (1C x7/10), 19.8 (1C x7/10), 19.9 (1C x3/10), 23.7 (1C x3/10), 23.8 (1C x7/10), 25.0 (1C x3/10), 25.8, 26.3 (1C x3/10), 26.4 (1C x7/10), 27.3 (1C x7/10), 28.5 (1C x3/10), 28.6 (1C x7/10), 29.7 (1C x7/10), 30.0 (1C x3/10), 30.2 (1C x3/10), 32.1 (1C x7/10), 33.0 (1C x7/10), 33.1 (1C x3/10), 33.2 (1C x3/10), 34.2, 35.2 (1C x7/10), 35.3 (1C x3/10), 35.9, 36.4 (1C x3/10), 38.0 (1C x3/10), 41.5 (1C x7/10), 52.7 (1C x3/10), 53.6 (1C x7/10), 67.0 (1C x7/10), 67.1 (1C x3/10), 81.5 (1C x3/10), 82.7 (1C x7/10), $219.2(1 \mathrm{C} \times 3 / 10), 221.6(1 \mathrm{C} \times 7 / 10)$, one ${ }^{13} \mathrm{C}\left\{{ }^{1} \mathrm{H}\right\}$ peak of $14 a$ was not observed. HRMS (ESITOF) $[\mathrm{M}+\mathrm{Na}]^{+} \mathrm{m} / \mathrm{z}$ : Calcd for $\mathrm{C}_{25} \mathrm{H}_{44} \mathrm{O}_{3} \mathrm{SiNa} 443.2957$; Found 443.2943.

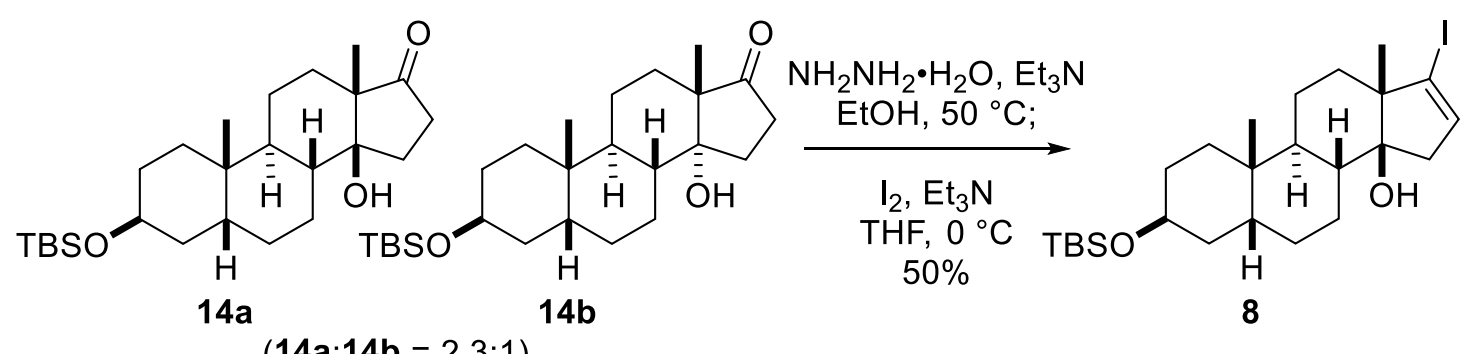

$(14 a: 14 b=2.3: 1)$

Vinyl iodide 8. $\mathrm{NH}_{2} \mathrm{NH}_{2} \cdot \mathrm{H}_{2} \mathrm{O}(7.2 \mathrm{~mL}, 0.15 \mathrm{~mol})$ and $\mathrm{Et} 3 \mathrm{~N}(21 \mathrm{~mL}, 0.15 \mathrm{~mol})$ were added to a solution of a 2.3:1 diastereomeric mixture of ketones $14 \mathbf{a}$ and $14 \mathbf{b}(3.14 \mathrm{~g}, 7.46 \mathrm{mmol})$ in $\mathrm{EtOH}(750 \mathrm{~mL})$ at room temperature. After being stirred at $50{ }^{\circ} \mathrm{C}$ for $13 \mathrm{~h}$, the reaction 
mixture was concentrated. A solution of $\mathrm{I}_{2}(5.73 \mathrm{~g}, 22.6 \mathrm{mmol})$ in THF $(50 \mathrm{~mL})$ was added dropwise to a solution of the residue and $\mathrm{Et}_{3} \mathrm{~N}(21 \mathrm{~mL}, 0.15 \mathrm{~mol})$ in THF $(300 \mathrm{~mL})$ at $0{ }^{\circ} \mathrm{C}$. After the reaction mixture was stirred at $0{ }^{\circ} \mathrm{C}$ for $40 \mathrm{~min}$, saturated aqueous $\mathrm{Na}_{2} \mathrm{~S}_{2} \mathrm{O}_{3}(150 \mathrm{~mL})$ and saturated aqueous $\mathrm{NaHCO}_{3}(100 \mathrm{~mL})$ were added to the mixture. The resultant mixture was extracted with EtOAc $(150 \mathrm{~mL}$ x3). The combined organic layers were washed with brine $(100 \mathrm{~mL})$, dried over $\mathrm{Na}_{2} \mathrm{SO}_{4}$, filtered, and concentrated. The residue was purified by flash column chromatography on silica gel $(250 \mathrm{~g}$, hexane/EtOAc $=1 / 0$ to $70 / 1$ to $10 / 1)$ to afford vinyl iodide $8(1.98 \mathrm{~g}, 3.73 \mathrm{mmol})$ in $50 \%$ yield: white foam. $[\alpha]_{\mathrm{D}}^{24}+12\left(c 0.54, \mathrm{CHCl}_{3}\right)$. IR (film) 3458, 2931, 2885, 2857, 1462, 1378, 1359, 1254, $1062 \mathrm{~cm}^{-1} .{ }^{1} \mathrm{H}$ NMR (500 MHz, $\left.\mathrm{CDCl}_{3}\right)$ $\delta 0.01(6 \mathrm{H}, \mathrm{s}, \mathrm{CH} 3$ of TBS), 0.80-1.05 (2H, m), 0.87 (9H, s, $t$-Bu of TBS), 0.93 (3H, s, H19), $1.03(3 \mathrm{H}, \mathrm{s}, \mathrm{H} 18), 1.15-1.26(3 \mathrm{H}, \mathrm{m}), 1.35-1.55(6 \mathrm{H}, \mathrm{m}), 1.61-1.97(6 \mathrm{H}, \mathrm{m}), 2.21(1 \mathrm{H}, \mathrm{d}, J=$ $16.6 \mathrm{~Hz}, \mathrm{H} 15 \mathrm{a}), 2.55(1 \mathrm{H}, \mathrm{d}, J=16.6 \mathrm{~Hz}, \mathrm{H} 15 \mathrm{~b}), 4.03(1 \mathrm{H}, \mathrm{s}, \mathrm{H} 3), 6.09(1 \mathrm{H}, \mathrm{s}, \mathrm{H} 16) .{ }^{13} \mathrm{C}\left\{{ }^{1} \mathrm{H}\right\}$ NMR $\left(125 \mathrm{MHz}, \mathrm{CDCl}_{3}\right) \delta-4.89,-4.87,17.9,18.0,19.7,21.1,24.0,25.8,26.5,28.7,29.7,34.2$, 35.1, 36.0, 36.5, 37.5, 41.4, 42.6, 54.7, 67.1, 82.5, 111.5, 133.5. HRMS (ESI-TOF) $[\mathrm{M}+\mathrm{Na}]^{+}$ $\mathrm{m} / \mathrm{z}$ : Calcd for $\mathrm{C}_{25} \mathrm{H}_{43} \mathrm{IO}_{2} \mathrm{SiNa} 553.1975$; Found 553.1955.

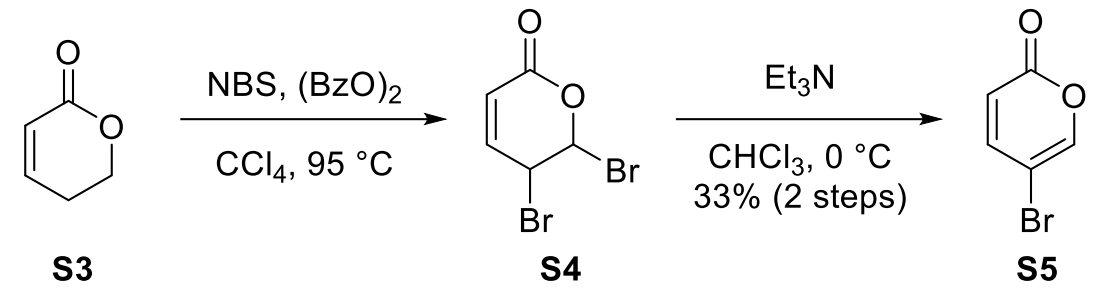

Bromide S5 [CAS: 19978-33-7]. ${ }^{\mathrm{S} 5} \quad N$-bromosuccinimide (NBS, $39.5 \mathrm{~g}, 222 \mathrm{mmol}$ ) and benzoyl peroxide $\left((\mathrm{BzO})_{2}, 75 \%\right.$ purity, $\left.1.36 \mathrm{~g}, 4.21 \mathrm{mmol}\right)$ were successively added to a solution of lactone $\mathbf{S 3}(9.0 \mathrm{~mL}, 0.11 \mathrm{~mol})$ in $\mathrm{CCl}_{4}(500 \mathrm{~mL})$ at room temperature. After being stirred at $95{ }^{\circ} \mathrm{C}$ for $14.5 \mathrm{~h}$, the reaction mixture was concentrated. The residue was purified by flash column chromatography on silica gel $(250 \mathrm{~g}$, hexane/EtOAc $=1 / 0$ to $3 / 1)$ to afford the crude dibromide $\mathbf{S 4}$ (24.6 g), which was used in the next reaction without further purification. $\mathrm{Et}_{3} \mathrm{~N}$ (74 $\mathrm{mL}, 0.53 \mathrm{~mol}$ ) was added to a solution of the above crude dibromide $\mathbf{S 4}$ (24.6 g) in $\mathrm{CHCl}_{3}(500 \mathrm{~mL})$ at $0{ }^{\circ} \mathrm{C}$. After being stirred at $0{ }^{\circ} \mathrm{C}$ for $4 \mathrm{~h}$, the reaction mixture was concentrated. The resulting mixture was filtered through a pad of silica gel $(150 \mathrm{~g}$, hexane/EtOAc = 1/1) and concentrated. The residue was purified by flash column chromatography on silica gel (500 g, hexane/EtOAc $=1 / 0$ to $30 / 1$ to 20/1) to afford bromide S5 $(6.31 \mathrm{~g}, 36.1 \mathrm{mmol})$ in $33 \%$ yield over 2 steps as white solid. The analytical data of S5 were identical to those reported previously. ${ }^{\mathrm{s} 6}$ 
<smiles>O=c1ccc(Br)co1</smiles>

S5

$$
\frac{\operatorname{Pd}\left(\mathrm{PPh}_{3}\right)_{4},\left(\mathrm{Me}_{3} \mathrm{Sn}\right)_{2}}{\underset{80 \%}{1,4 \text {-dioxane, } 100{ }^{\circ} \mathrm{C}}}
$$<smiles>C[AsH2]c1ccc(=O)oc1</smiles>

7

2-Pyrone 7 [CAS: 181866-08-0]. ${ }^{\mathrm{S} 6} \quad \mathrm{Pd}\left(\mathrm{PPh}_{3}\right)_{4}(1.91 \mathrm{~g}, 1.65 \mathrm{mmol})$ and $(\mathrm{Me} 3 \mathrm{Sn})_{2}(4.4 \mathrm{~mL}, 21$ mmol) were added to a solution of bromide S5 (2.84 g, $16.2 \mathrm{mmol})$ in 1,4-dioxane ( $41 \mathrm{~mL})$, and then the mixture was degassed by the freeze-thaw procedure (x3). After being stirred at $100{ }^{\circ} \mathrm{C}$ for $12 \mathrm{~h}$, the reaction mixture was concentrated. The residue was purified by flash column chromatography on silica gel $(150 \mathrm{~g}$, hexane/EtOAc $=1 / 0$ to $20 / 1$ to $10 / 1)$ to afford 2pyrone 7 (3.34 g, $12.9 \mathrm{mmol}$ ) in 80\% yield: colorless oil. IR (film) 2958, 2919, 2850, 1739, 1716, 1522, 1411, 1341, 1216, $1131 \mathrm{~cm}^{-1} .{ }^{1} \mathrm{H}$ NMR (400 MHz, C6 6$) \delta-0.05\left(9 \mathrm{H}, \mathrm{s}, \mathrm{CH}_{3}\right.$ of $\left.\mathrm{SnMe}_{3}\right), 6.04(1 \mathrm{H}, \mathrm{d}, J=9.2 \mathrm{~Hz}, \mathrm{H} 23), 6.45(1 \mathrm{H}, \mathrm{dd}, J=9.2,1.6 \mathrm{~Hz}, \mathrm{H} 22), 6.66(1 \mathrm{H}, \mathrm{s}, \mathrm{H} 21)$. ${ }^{13} \mathrm{C}\left\{{ }^{1} \mathrm{H}\right\}$ NMR $\left(100 \mathrm{MHz}, \mathrm{C}_{6} \mathrm{D}_{6}\right) \delta-10.0,112.0,117.4,147.1,155.0,160.8$. HRMS (ESI-TOF) $[\mathrm{M}+\mathrm{Na}]^{+} \mathrm{m} / \mathrm{z}$ : Calcd for $\mathrm{C}_{8} \mathrm{H}_{12} \mathrm{O}_{2} \mathrm{SnNa} 282.9751$; Found 282.9756 .

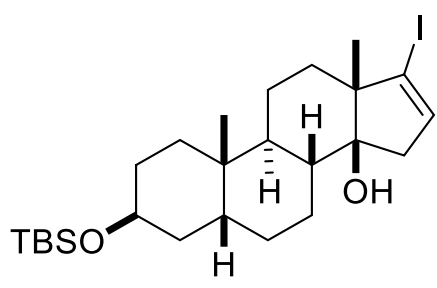

8

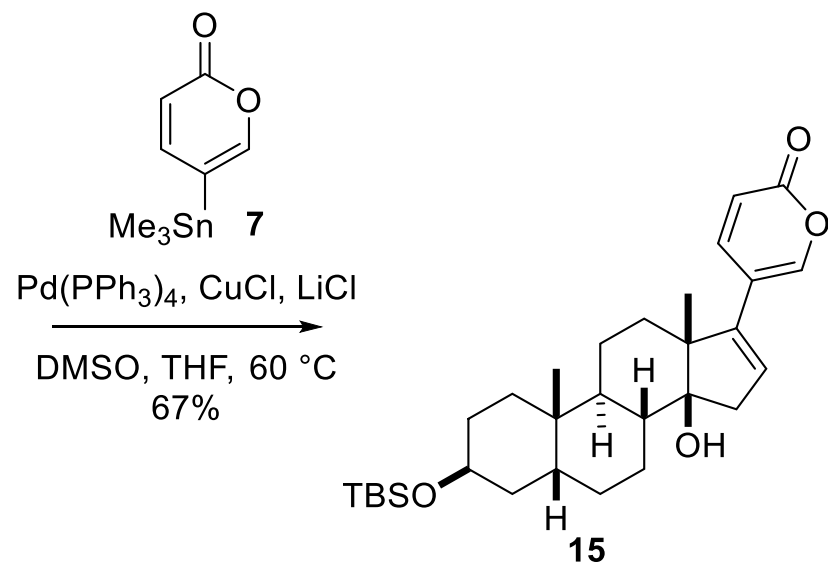

Pentacycle 15. $\mathrm{Pd}\left(\mathrm{PPh}_{3}\right)_{4}(3.24 \mathrm{~g}, 2.80 \mathrm{mmol}), \mathrm{CuCl}(2.75 \mathrm{~g}, 27.8 \mathrm{mmol})$, and $\mathrm{LiCl}(1.41 \mathrm{~g}$, $33.3 \mathrm{mmol})$ were added to a mixture of vinyl iodide $\mathbf{8}(2.95 \mathrm{~g}, 5.56 \mathrm{mmol})$ and 2-pyrone 7 (4.80 $\mathrm{g}, 18.5 \mathrm{mmol})$. DMSO (49 $\mathrm{mL})$ and THF $(6.2 \mathrm{~mL})$ were added to the mixture, and then the mixture was degassed by the freeze-thaw procedure (x3). After being stirred at $60{ }^{\circ} \mathrm{C}$ for 13 $\mathrm{h}$, the reaction mixture was filtered through a pad of silica gel $(200 \mathrm{~g}$, hexane/EtOAc $=1 / 1)$ and concentrated. Then $\mathrm{pH} 7$ phosphate buffer $(200 \mathrm{~mL})$ was added to the residue. The resultant mixture was extracted with $\mathrm{Et}_{2} \mathrm{O}(200 \mathrm{~mL} \times 5)$. The combined organic layers were washed with brine $(100 \mathrm{~mL})$, dried over $\mathrm{Na}_{2} \mathrm{SO}_{4}$, filtered, and concentrated. The residue was purified by flash column chromatography (a column consecutively packed with silica gel $150 \mathrm{~g}$ and 10 $\mathrm{wt} \% \mathrm{KF}$ contained silica gel $50 \mathrm{~g}$, hexane/EtOAc $=1 / 0$ to $10 / 1$ to $2 / 1$ to $1 / 1)$ to afford pentacycle $15(1.87 \mathrm{~g}, 3.75 \mathrm{mmol})$ in $67 \%$ yield: white solid. m.p. $260{ }^{\circ} \mathrm{C} .[\alpha]_{\mathrm{D}}{ }^{27}-58\left(c 0.60, \mathrm{CHCl}_{3}\right)$. IR (film) $3454,2935,2880,2856,1727,1694,1528,1444,1374,1252,1070,833 \mathrm{~cm}^{-1} .{ }^{1} \mathrm{H}$ NMR $\left(400 \mathrm{MHz}, \mathrm{CDCl}_{3}\right) \delta 0.01\left(6 \mathrm{H}, \mathrm{s}, \mathrm{CH}_{3}\right.$ of TBS), $0.87(9 \mathrm{H}, \mathrm{s}, t$-Bu of TBS), $0.94(3 \mathrm{H}, \mathrm{s}, \mathrm{H} 19)$, 1.03-1.09 (1H, m), 1.12 (3H, s, H18), 1.17-1.28 (4H, m), 1.35-1.49 (4H, m), 1.50-1.58 (2H, m), 
1.62-1.73 (1H, m), 1.77-1.83 (3H, m), 1.86-1.93 (2H, m), $2.24(1 \mathrm{H}, \mathrm{dd}, J=17.4,3.2 \mathrm{~Hz}, \mathrm{H} 15 \mathrm{a})$, $2.68(1 \mathrm{H}, \mathrm{dd}, J=17.4,1.8 \mathrm{~Hz}, \mathrm{H} 15 \mathrm{~b}), 4.04(1 \mathrm{H}, \mathrm{s}, \mathrm{H} 3), 5.77(1 \mathrm{H}, \mathrm{dd}, J=3.2,1.8 \mathrm{~Hz}, \mathrm{H} 16)$, $6.31(1 \mathrm{H}, \mathrm{dd}, J=9.8,1.2 \mathrm{~Hz}, \mathrm{H} 23), 7.38(1 \mathrm{H}, \mathrm{dd}, J=9.8,2.8 \mathrm{~Hz}, \mathrm{H} 22), 7.438-7.444(1 \mathrm{H}, \mathrm{m}$, $\mathrm{H} 21) .{ }^{13} \mathrm{C}\left\{{ }^{1} \mathrm{H}\right\} \mathrm{NMR}\left(100 \mathrm{MHz}, \mathrm{CDCl}_{3}\right) \delta-4.92,-4.88,16.2,18.0,19.8,21.2,24.0,25.8,26.5$, 28.7, 29.7, 34.2, 35.1, 35.9, 36.1, 38.6, 39.8, 40.9, 52.3, 67.1, 85.8, 115.9, 116.3, 125.0, 144.3, 145.1, 147.5, 161.2. HRMS (ESI-TOF) $[\mathrm{M}+\mathrm{Na}]^{+} \mathrm{m} / \mathrm{z}$ : Calcd for $\mathrm{C}_{30} \mathrm{H}_{46} \mathrm{O}_{4} \mathrm{SiNa} 521.3058$; Found 521.3060.
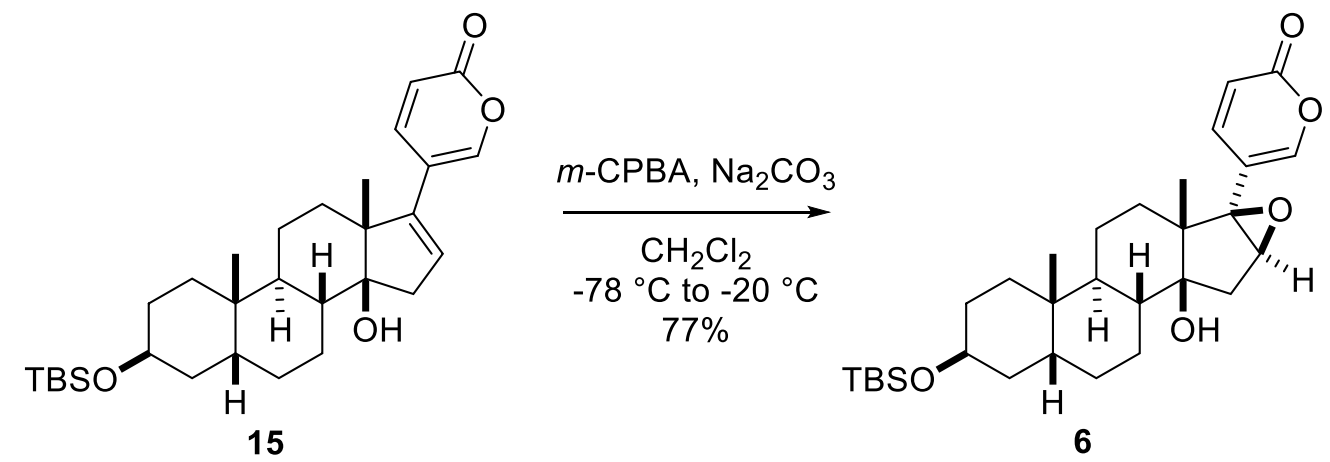

Epoxide 6. $m$-Chloroperoxybenzoic acid ( $m$-CPBA, 77\% purity, $965 \mathrm{mg}, 4.31 \mathrm{mmol}$ ) was added to a solution of pentacycle $15(1.65 \mathrm{~g}, 3.31 \mathrm{mmol})$ and $\mathrm{Na}_{2} \mathrm{CO}_{3}(465 \mathrm{mg}, 4.39 \mathrm{mmol})$ in $\mathrm{CH}_{2} \mathrm{Cl}_{2}(83 \mathrm{~mL})$ at $-78{ }^{\circ} \mathrm{C}$. After the reaction mixture was stirred at $-20{ }^{\circ} \mathrm{C}$ for $5 \mathrm{~h}$, saturated aqueous $\mathrm{Na}_{2} \mathrm{~S}_{2} \mathrm{O}_{3}(50 \mathrm{~mL})$ was added to the mixture. The resultant mixture was extracted with EtOAc $(50 \mathrm{~mL} \times 3)$. The combined organic layers were washed with saturated aqueous $\mathrm{NaHCO}_{3}(20 \mathrm{~mL})$, dried over $\mathrm{Na}_{2} \mathrm{SO}_{4}$, filtered, and concentrated. The residue was purified by flash column chromatography on silica gel $(100 \mathrm{~g}$, hexane/EtOAc $=1 / 0$ to $10 / 1$ to $5 / 1$ to $2 / 1)$ to afford epoxide $\mathbf{6}$ (1.32 g, $2.56 \mathrm{mmol})$ in 77\% yield. The structure of $\mathbf{6}$ was determined by the X-ray crystallographic analysis (Page S39): colorless prism crystal (recrystallization from hexane/EtOAc by vapor diffusion method). m.p. $240-242{ }^{\circ} \mathrm{C} .[\alpha]_{\mathrm{D}}{ }^{25}+10\left(c 0.85, \mathrm{CHCl}_{3}\right)$. IR (film) 3535, 3491, 3080, 2925, 2886, 2859, 1730, 1713, 1539, 1453, 1376, 1254, 1161, 1121 $\mathrm{cm}^{-1} .{ }^{1} \mathrm{H}$ NMR (400 MHz, C6 $\mathrm{D}_{6}$ ) $\delta 0.105$ (3H, s, CH 3 of TBS), 0.112 (3H, s, CH3 of TBS), 0.41$0.48(1 \mathrm{H}, \mathrm{m}), 0.82-0.94(2 \mathrm{H}, \mathrm{m}), 0.88(3 \mathrm{H}, \mathrm{s}, \mathrm{H} 19), 0.89(3 \mathrm{H}, \mathrm{s}, \mathrm{H} 18), 1.02(9 \mathrm{H}, \mathrm{s}, t$-Bu of TBS), 1.05-1.13 (1H, m), 1.19-1.24 (3H, m), 1.29-1.33 (1H, m), 1.45-1.65 (5H, m), 1.69-1.86 $(3 \mathrm{H}, \mathrm{m}), 1.87-1.96(2 \mathrm{H}, \mathrm{m}), 2.10-2.14(1 \mathrm{H}, \mathrm{m}), 2.99(1 \mathrm{H}, \mathrm{s}, \mathrm{H} 16), 3.28(1 \mathrm{H}, \mathrm{brs}, \mathrm{OH}), 4.11(1 \mathrm{H}$, brs, H3), $5.89(1 \mathrm{H}, \mathrm{dd}, J=9.6,1.2 \mathrm{~Hz}, \mathrm{H} 23), 6.61(1 \mathrm{H}, \mathrm{dd}, J=9.6,2.7 \mathrm{~Hz}, \mathrm{H} 22), 6.77(1 \mathrm{H}, \mathrm{dd}$, $J=2.7,1.2 \mathrm{~Hz}, \mathrm{H} 21) .{ }^{13} \mathrm{C}\left\{{ }^{1} \mathrm{H}\right\} \mathrm{NMR}\left(100 \mathrm{MHz}, \mathrm{C}_{6} \mathrm{D}_{6}\right) \delta-4.7,-4.6,13.9,18.3,20.0,21.4,24.2$, 26.1, 26.9, 29.2, 30.1, 34.6, 34.8, 35.0, 35.4, 35.9, 36.4, 40.8, 46.4, 61.2, 67.7, 71.2, 80.6, 113.0, 115.9, 143.0, 151.1, 159.9. HRMS (ESI-TOF) $[\mathrm{M}+\mathrm{Na}]^{+} \mathrm{m} / \mathrm{z}$ : Calcd for $\mathrm{C}_{30} \mathrm{H}_{46} \mathrm{O}_{5} \mathrm{SiNa}$ 537.3007; Found 537.3005. 


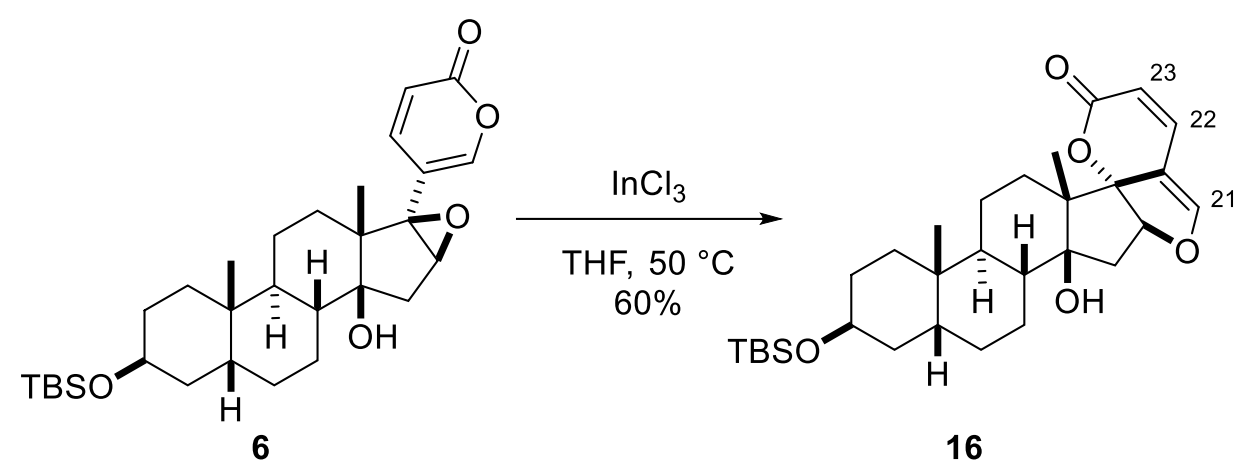

Hexacycle 16. A solution of $\mathrm{InCl}_{3}(40.1 \mathrm{mg}, 181 \mu \mathrm{mol})$ in $\mathrm{THF}(0.60 \mathrm{~mL})$ was added to a solution of epoxide $6(24.6 \mathrm{mg}, 47.8 \mu \mathrm{mol})$ in THF $(1.0 \mathrm{~mL})$ at room temperature. After the reaction mixture was stirred at $50{ }^{\circ} \mathrm{C}$ for $5 \mathrm{~h}$, saturated aqueous $\mathrm{NaHCO}_{3}(5 \mathrm{~mL})$ was added to the mixture at room temperature. The resultant mixture was extracted with EtOAc $(5 \mathrm{~mL} \times 3)$. The combined organic layers were washed with brine $(5 \mathrm{~mL})$, dried over $\mathrm{Na}_{2} \mathrm{SO}_{4}$, filtered, and concentrated. The residue was purified by flash column chromatography on silica gel $(5 \mathrm{~g}$, hexane/EtOAc $=1 / 0$ to $2 / 1$ to $1 / 1)$ to afford alcohol $16(14.9 \mathrm{mg}, 28.9 \mu \mathrm{mol})$ in $60 \%$ yield: white solid. m.p. $235-237{ }^{\circ} \mathrm{C}$. $[\alpha]_{\mathrm{D}^{23}}+24\left(c 0.37, \mathrm{CHCl}_{3}\right)$. IR (film) 3398, 2932, 2858, 1685, $1626,1452,1253,1128,1066 \mathrm{~cm}^{-1} .{ }^{1} \mathrm{H} \mathrm{NMR}\left(500 \mathrm{MHz}, \mathrm{CDCl}_{3}\right) \delta 0.01\left(3 \mathrm{H}, \mathrm{s}, \mathrm{CH}_{3}\right.$ of TBS), $0.02\left(3 \mathrm{H}, \mathrm{s}, \mathrm{CH} \mathrm{H}_{3}\right.$ of TBS), $0.88(9 \mathrm{H}, \mathrm{s}, t-\mathrm{Bu}$ of TBS), $0.91(3 \mathrm{H}, \mathrm{s}, \mathrm{H} 19), 1.04$ (3H, s, H18), $1.08-$ $1.87(17 \mathrm{H}, \mathrm{m}), 2.08(1 \mathrm{H}, \mathrm{d}, J=15.2 \mathrm{~Hz}, \mathrm{H} 15 \mathrm{a}), 2.50(1 \mathrm{H}, \mathrm{dd}, J=15.2,6.6 \mathrm{~Hz}, \mathrm{H} 15 \mathrm{~b}), 4.05$ (1H, brs, H3), $5.18(1 \mathrm{H}, \mathrm{d}, J=6.6 \mathrm{~Hz}, \mathrm{H} 16), 5.72(1 \mathrm{H}, \mathrm{d}, J=9.4 \mathrm{~Hz}, \mathrm{H} 23), 6.75(1 \mathrm{H}, \mathrm{s}, \mathrm{H} 21)$, $7.29(1 \mathrm{H}, \mathrm{d}, J=9.4 \mathrm{~Hz}, \mathrm{H} 22) .{ }^{13} \mathrm{C}\left\{{ }^{1} \mathrm{H}\right\} \mathrm{NMR}\left(125 \mathrm{MHz}, \mathrm{CDCl}_{3}\right) \delta-4.9,16.3,18.1,20.6,20.8$, 23.7, 25.8, 26.6, 28.6, 29.5, 33.2, 34.2, 35.2, 35.5, 35.9, 37.0, 42.0, 54.3, 67.1, 86.8, 93.3, 101.6, 111.8, 115.4, 136.7, 149.5, 163.6. HRMS (ESI-TOF) $[\mathrm{M}+\mathrm{Na}]^{+} \mathrm{m} / \mathrm{z}$ : Calcd for $\mathrm{C}_{30} \mathrm{H}_{46} \mathrm{O}_{5} \mathrm{SiNa}$ 537.3007; Found 537.2983.

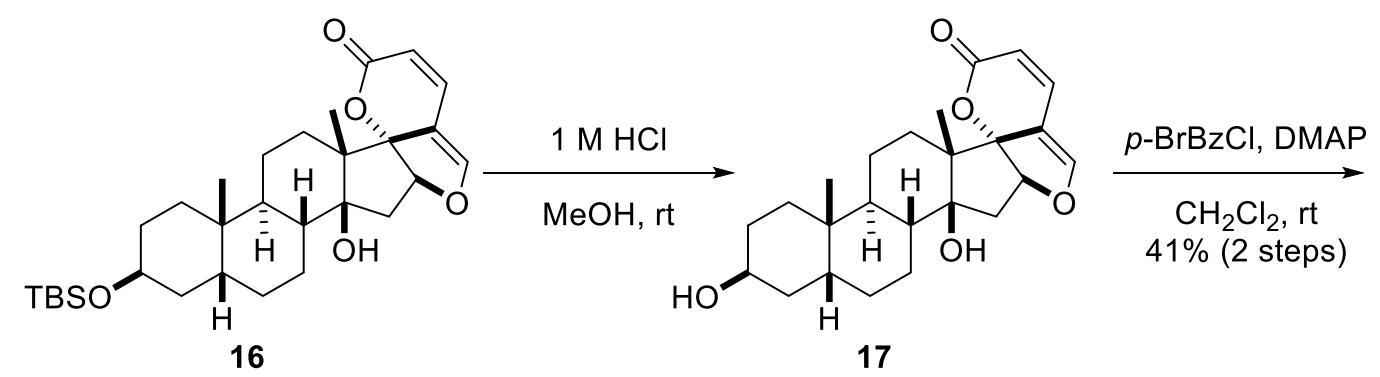<smiles>CC12CCC(OC(=O)c3ccc(Br)cc3)CC1CCC1C2CCC23OC(=O)C=CC2=COCC13O</smiles>

p-Bromobenzoate 18. $1 \mathrm{M}$ aqueous $\mathrm{HCl}(270 \mu \mathrm{L}, 270 \mu \mathrm{mol})$ was added to a solution of alcohol $16(7.4 \mathrm{mg}, 14 \mu \mathrm{mol})$ in $\mathrm{MeOH}(3.2 \mathrm{~mL})$ at room temperature. After the reaction 
mixture was stirred at room temperature for $1.5 \mathrm{~h}$, saturated aqueous $\mathrm{NaHCO}_{3}(3 \mathrm{~mL})$ was added to the mixture at $0{ }^{\circ} \mathrm{C}$. The resultant mixture was extracted with EtOAc $(3 \mathrm{~mL} \times 4)$. The combined organic layers were washed with brine $(3 \mathrm{~mL})$, dried over $\mathrm{Na}_{2} \mathrm{SO}_{4}$, filtered, and concentrated. The residue was purified by flash column chromatography on silica gel $(1 \mathrm{~g}$, hexane/EtOAc $=1 / 0$ to $1 / 1$ to $1 / 2)$ to afford the crude diol $17(4.8 \mathrm{mg})$, which was used in the next reaction without further purification: colorless amorphous. HRMS (ESI-TOF) $[\mathrm{M}+\mathrm{Na}]^{+}$ $\mathrm{m} / \mathrm{z}$ : Calcd for $\mathrm{C}_{24} \mathrm{H}_{32} \mathrm{O}_{5} \mathrm{Na}$ 423.2142; Found 423.2157.

$p$ - $\mathrm{BrBzCl}(13.2 \mathrm{mg}, 60.1 \mu \mathrm{mol})$ was added to a solution of the above crude diol 17 (4.8 $\mathrm{mg})$ and $N, N$-dimethyl-4-aminopyridine (DMAP, $7.8 \mathrm{mg}, 64 \mu \mathrm{mol})$ in $\mathrm{CH}_{2} \mathrm{Cl}_{2}(0.40 \mathrm{~mL}$ ) at room temperature. After the reaction mixture was stirred at room temperature for $14 \mathrm{~h}$, DMAP (7.5 $\mathrm{mg}, 61 \mu \mathrm{mol})$ and $p$ - $\mathrm{BrBzCl}(12.5 \mathrm{mg}, 57.0 \mu \mathrm{mol})$ were successively added to the mixture. After the reaction mixture was stirred at room temperature for $3 \mathrm{~h}$, saturated aqueous $\mathrm{NaHCO}_{3}$ $(3 \mathrm{~mL})$ was added to the mixture at $0{ }^{\circ} \mathrm{C}$. The resultant mixture was extracted with EtOAc ( 3 $\mathrm{mL} \times 4)$. The combined organic layers were washed with brine $(3 \mathrm{~mL})$, dried over $\mathrm{Na}_{2} \mathrm{SO}_{4}$, filtered, and concentrated. The residue was purified by flash column chromatography on silica gel $(1 \mathrm{~g}$, hexane/EtOAc $=1 / 0$ to $2 / 1$ to $1 / 2)$ to afford $p$-bromobenzoate $18(3.3 \mathrm{mg}, 5.7 \mu \mathrm{mol})$ in $41 \%$ yield over 2 steps. The structure of $\mathbf{1 8}$ was determined by the X-ray crystallographic analysis (Page S40): colorless plate crystal (recrystallization from hexane/EtOAc by vapor diffusion method). m.p. $201-203{ }^{\circ} \mathrm{C}$. $[\alpha]_{\mathrm{D}}{ }^{24}+6.4\left(c\right.$ 0.17, $\left.\mathrm{CHCl}_{3}\right)$. IR (film) 3496, 2938, 2872 , $1711,1633,1453,1389,1277,1115,1012 \mathrm{~cm}^{-1} .{ }^{1} \mathrm{H}$ NMR (500 MHz, DMSO-d6) $\delta$ 0.86-2.09 (18H, m), 0.917 (3H, s, H18), 0.924 (3H, s, H19), 2.58 (1H, dd, $J=15.5,6.3 \mathrm{~Hz}, \mathrm{H} 15 \mathrm{a}), 3.98$ $(1 \mathrm{H}, \mathrm{s}, \mathrm{OH}), 5.07(1 \mathrm{H}, \mathrm{d}, J=6.3 \mathrm{~Hz}, \mathrm{H} 16), 5.23(1 \mathrm{H}, \mathrm{brs}, \mathrm{H} 3), 5.57(1 \mathrm{H}, \mathrm{d}, J=9.2 \mathrm{~Hz}, \mathrm{H} 23)$, 7.07 (1H, s, H21), $7.50(1 \mathrm{H}, \mathrm{d}, J=9.2 \mathrm{~Hz}, \mathrm{H} 22), 7.75$ (2H, d, $J=7.8 \mathrm{~Hz}$, aromatic), 7.89 (2H, $\mathrm{d}, J=7.8 \mathrm{~Hz}$, aromatic). ${ }^{13} \mathrm{C}\left\{{ }^{1} \mathrm{H}\right\} \mathrm{NMR}(100 \mathrm{MHz}$, DMSO-d6) $\delta 16.8,20.1,20.4,23.6,24.5$, 26.1, 30.0, 30.5, 32.6, 34.4, 34.9, 36.4, 36.9, 41.3, 53.8, 71.5, 84.7, 92.6, 101.9, 111.2, 113.5, 127.2, 129.6, 131.1, 132.0, 138.4, 151.2, 163.2, 164.4. HRMS (ESI-TOF) $[\mathrm{M}+\mathrm{Na}]^{+} \mathrm{m} / \mathrm{z}$ : Calcd for $\mathrm{C}_{31} \mathrm{H}_{35} \mathrm{BrO}_{6} \mathrm{Na} 605.1509$ and 607.1489; Found 605.1493 and 607.1489.
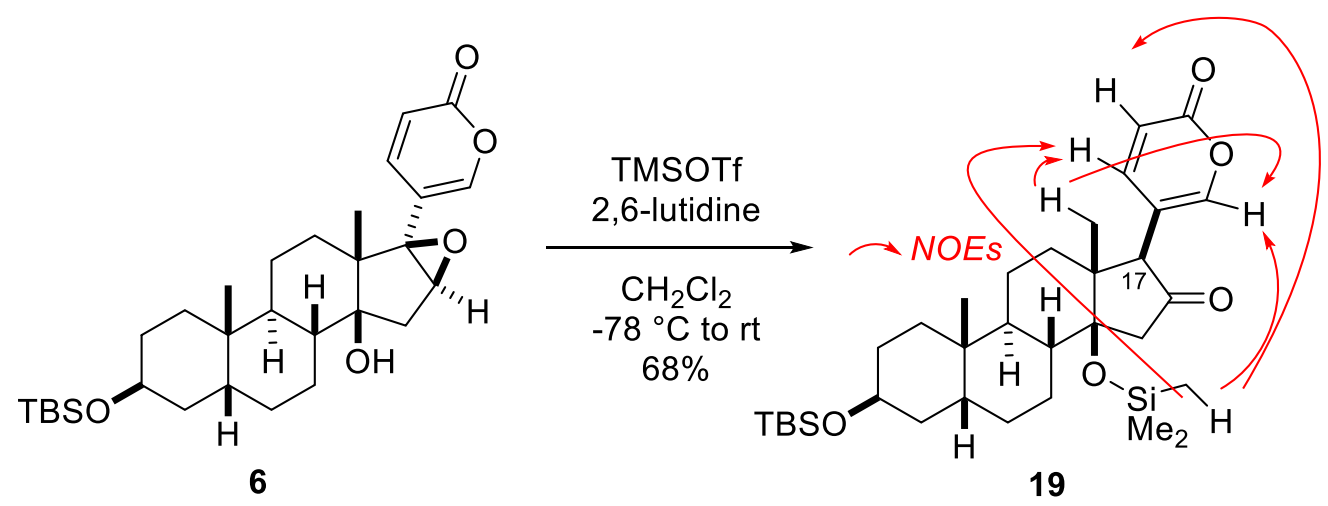

Ketone 19. TMSOTf (1.1 mL, $6.1 \mathrm{mmol})$ was added to a solution of epoxide 6 (1.32 $\mathrm{g}, 2.56$ mmol) and 2,6-lutidine $(1.5 \mathrm{~mL}, 13 \mathrm{mmol})$ in $\mathrm{CH}_{2} \mathrm{Cl}_{2}(26 \mathrm{~mL})$ at $-78{ }^{\circ} \mathrm{C}$. After the reaction mixture was stirred at room temperature for $1 \mathrm{~h}$, saturated aqueous $\mathrm{NaHCO}_{3}(50 \mathrm{~mL})$ was added to the mixture at $-78{ }^{\circ} \mathrm{C}$. The resultant mixture was extracted with EtOAc $(50 \mathrm{~mL} \times 3)$. The 
combined organic layers were washed with brine $(50 \mathrm{~mL})$, dried over $\mathrm{Na}_{2} \mathrm{SO}_{4}$, filtered, and concentrated. The residue was purified by flash column chromatography on silica gel $(50 \mathrm{~g}$, hexane $/$ EtOAc $=1 / 0$ to $10 / 1$ to $5 / 1)$ to afford ketone $19(1.03 \mathrm{~g}, 1.75 \mathrm{mmol})$ in $68 \%$ yield. The C17-stereochemistry of $\mathbf{1 9}$ was determined by the NOE experiment: white solid. m.p. 138$140{ }^{\circ} \mathrm{C} .[\alpha]_{\mathrm{D}}{ }^{25}+44\left(c 0.54, \mathrm{CHCl}_{3}\right)$. IR (film) 2930, 2858, 1747, 1540, 1448, 1380, 1253, 1062 , 984, $838 \mathrm{~cm}^{-1} .{ }^{1} \mathrm{H}$ NMR (400 MHz, $\left.\mathrm{C}_{6} \mathrm{D}_{6}\right) \delta-0.01$ (9H, s, CH3 of TMS), $0.13\left(3 \mathrm{H}, \mathrm{s}, \mathrm{CH}_{3}\right.$ of TBS), 0.14 (3H, s, $\mathrm{CH}_{3}$ of TBS), 0.62 (3H, s, H18), 0.85-1.12 (4H, m), 0.87 (3H, s, H19), 1.04 $(9 \mathrm{H}, \mathrm{s}, t-\mathrm{Bu}$ of TBS), 1.16-1.51 $(7 \mathrm{H}, \mathrm{m}), 1.55-1.82(5 \mathrm{H}, \mathrm{m}), 1.91-1.94(1 \mathrm{H}, \mathrm{m}), 2.14(1 \mathrm{H}, \mathrm{d}, J$ $=17.4 \mathrm{~Hz}, \mathrm{H} 15 \mathrm{a}), 2.36$ (1H, s, H17), $2.42(1 \mathrm{H}, \mathrm{d}, J=17.4 \mathrm{~Hz}, \mathrm{H} 15 \mathrm{~b}), 4.08$ (1H, brs, H3), 6.00 $(1 \mathrm{H}, \mathrm{dd}, J=9.6,1.4 \mathrm{~Hz}, \mathrm{H} 23), 6.72(1 \mathrm{H}, \mathrm{brs}, \mathrm{H} 21), 6.74(1 \mathrm{H}, \mathrm{dd}, J=9.6,2.8 \mathrm{~Hz}, \mathrm{H} 22) .{ }^{13} \mathrm{C}\left\{{ }^{1} \mathrm{H}\right\}$ NMR (100 MHz, $\left.\mathrm{C}_{6} \mathrm{D}_{6}\right) \delta-4.7,-4.6,2.8,18.3,18.4,20.6,23.6,24.1,26.1,26.5,28.8,30.1$, 34.6, 35.9, 36.2, 37.2, 39.9, 41.5, 47.97, 48.04, 61.4, 67.6, 88.3, 115.3, 116.1, 146.1, 151.8, 160.4, 214.1. HRMS (ESI-TOF) $[\mathrm{M}+\mathrm{Na}]^{+} \mathrm{m} / \mathrm{z}$ : Calcd for $\mathrm{C}_{33} \mathrm{H}_{54} \mathrm{O}_{5} \mathrm{Si}_{2} \mathrm{Na}$ 609.3402; Found 609.3374 .

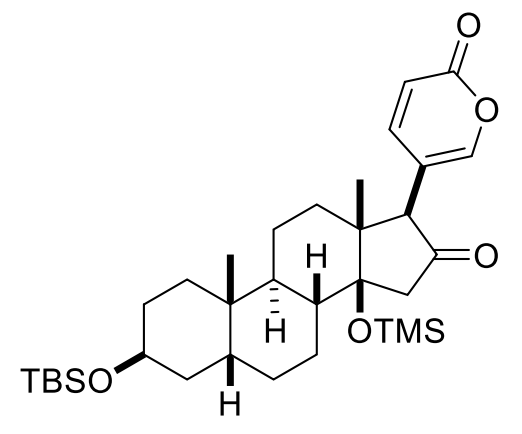

19

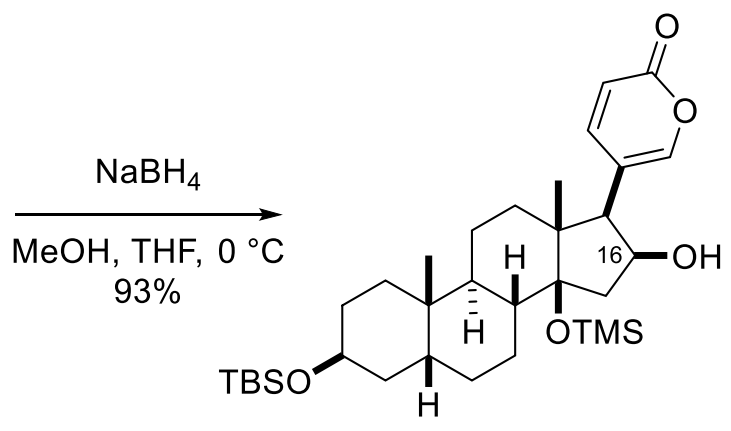

24

Alcohol 24. $\mathrm{NaBH}_{4}(80.6 \mathrm{mg}, 2.13 \mathrm{mmol})$ was added to a solution of ketone $\mathbf{1 9}(1.03 \mathrm{~g}, 1.75$ $\mathrm{mmol})$ in $\mathrm{MeOH}(17 \mathrm{~mL})$ and $\mathrm{THF}(17 \mathrm{~mL})$ at $0{ }^{\circ} \mathrm{C}$. After the reaction mixture was stirred at $0{ }^{\circ} \mathrm{C}$ for $1.5 \mathrm{~h}$, saturated aqueous $\mathrm{NH}_{4} \mathrm{Cl}(50 \mathrm{~mL})$ was added to the mixture. The resultant mixture was extracted with EtOAc $(50 \mathrm{~mL} \times 3)$. The combined organic layers were washed with brine $(20 \mathrm{~mL})$, dried over $\mathrm{Na}_{2} \mathrm{SO}_{4}$, filtered, and concentrated. The residue was purified by flash column chromatography on silica gel ( $50 \mathrm{~g}$, hexane/EtOAc $=1 / 0$ to $5 / 1$ to $3 / 1)$ to afford alcohol 24 (954 mg, $1.62 \mathrm{mmol}$ ) in 93\% yield. The C16-stereochemistry of $\mathbf{2 4}$ was determined by converting 24 to bufogenin B (2): colorless oil. [ $\alpha]_{\mathrm{D}}{ }^{24}+13\left(c 0.70, \mathrm{CHCl}_{3}\right)$. IR (film) 3460, 2931, 2859, 1714, 1634, 1538, 1447, 1379, 1251, 1116, 1061, $994 \mathrm{~cm}^{-1} .{ }^{1} \mathrm{H}$ NMR (400 MHz, $\left.\mathrm{CDCl}_{3}\right) \delta 0.02\left(6 \mathrm{H}, \mathrm{s}, \mathrm{CH}_{3}\right.$ of TBS), $0.26\left(9 \mathrm{H}, \mathrm{s}, \mathrm{CH}_{3}\right.$ of TMS $), 0.81(3 \mathrm{H}, \mathrm{s}), 0.88(9 \mathrm{H}, \mathrm{s}, t-\mathrm{Bu}$ of TBS), $0.91(3 \mathrm{H}, \mathrm{s}), 1.03-1.87(17 \mathrm{H}, \mathrm{m}), 1.93(1 \mathrm{H}, \mathrm{d}, J=14.6 \mathrm{~Hz}, \mathrm{H} 15 \mathrm{a}), 2.24(1 \mathrm{H}, \mathrm{dd}, J=$ 14.6, 6.0 Hz, H15b), 2.54 (1H, d, J = 6.4 Hz, H17), 4.05 (1H, brs, H3), 4.29 (1H, dd, $J=6.4$, $6.0 \mathrm{~Hz}, \mathrm{H} 16), 6.21(1 \mathrm{H}, \mathrm{d}, J=9.8 \mathrm{~Hz}, \mathrm{H} 23), 7.30(1 \mathrm{H}, \mathrm{brs}, \mathrm{H} 21), 7.56(1 \mathrm{H}, \mathrm{dd}, J=9.8,1.7 \mathrm{~Hz}$, H22). ${ }^{13} \mathrm{C}\left\{{ }^{1} \mathrm{H}\right\}$ NMR (100 MHz, $\left.\mathrm{C}_{6} \mathrm{D}_{6}\right) \delta-4.7,-4.6,3.1,18.4,18.7,20.9,23.9,24.1,26.1,26.8$, 28.9, 30.2, 34.7, 36.0, 36.3, 37.3, 41.4, 42.8, 43.1, 49.7, 59.1, 67.7, 73.7, 92.7, 113.2, 117.4, 149.1, 150.4, 161.5. HRMS (ESI-TOF) $[\mathrm{M}+\mathrm{Na}]^{+} \mathrm{m} / \mathrm{z}$ : Calcd for $\mathrm{C}_{33} \mathrm{H}_{56} \mathrm{O}_{5} \mathrm{Si}_{2} \mathrm{Na} 611.3558$; Found 611.3537. 

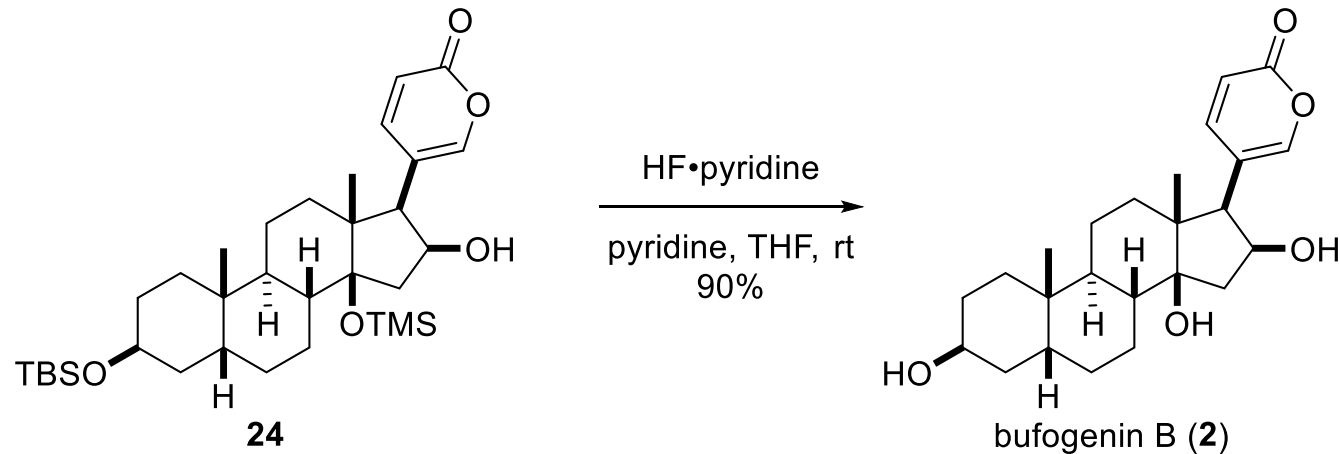

Bufogenin B (2) [CAS: 465-19-0]. A mixture of HF (70 wt $\%$ pyridine solution, $1.6 \mathrm{~mL}, 61$ mmol) and pyridine $(1.6 \mathrm{~mL})$ was added to a solution of alcohol $24(183 \mathrm{mg}, 311 \mu \mathrm{mol})$ in $\mathrm{THF}$ $(3.1 \mathrm{~mL})$ at room temperature. After the reaction mixture was stirred at room temperature for $77 \mathrm{~h}$, saturated aqueous $\mathrm{NaHCO}_{3}(30 \mathrm{~mL})$ was added to the mixture. The resultant mixture was extracted with EtOAc $(30 \mathrm{~mL} x 4)$. The combined organic layers were washed with brine $(10 \mathrm{~mL})$, dried over $\mathrm{Na}_{2} \mathrm{SO}_{4}$, filtered, and concentrated. The residue was purified by flash column chromatography on silica gel ( $5 \mathrm{~g}$, hexane/EtOAc $=1 / 1$ to $1 / 5$ to $1 / 2$ then $\mathrm{CHCl}_{3} / \mathrm{MeOH}$ $=5 / 1)$ to afford bufogenin B (2) $(113 \mathrm{mg}, 281 \mu \mathrm{mol})$ in $90 \%$ yield: colorless oil. $[\alpha]_{\mathrm{D}}{ }^{17}+44(c$ 0.54, DMSO). IR (film) 3397, 2930, 2861, 1712, 1631, 1537, 1448, 1379, 1258, $1029 \mathrm{~cm}^{-1} .{ }^{1} \mathrm{H}$ NMR (500 MHz, DMSO-d6) $\delta 0.64$ (3H, s, H18), 0.85 (3H, s, H19), 1.02-1.84 (18H, m), 2.44 (1H, dd, $J=14.4,8.3 \mathrm{~Hz}, \mathrm{H} 15 \mathrm{a}), 2.63$ (1H, d, $J=8.6 \mathrm{~Hz}, \mathrm{H} 17), 3.89$ (1H, brs, H3), 4.16 (1H, s, OH), $4.19(1 \mathrm{H}$, brs, OH $), 4.41-4.44(1 \mathrm{H}, \mathrm{m}, \mathrm{H} 16), 4.52(1 \mathrm{H}, \mathrm{d}, J=4.1 \mathrm{~Hz}, \mathrm{OH}), 6.12(1 \mathrm{H}, \mathrm{d}$, $J=9.8 \mathrm{~Hz}, \mathrm{H} 23), 7.47(1 \mathrm{H}, \mathrm{d}, J=2.3 \mathrm{~Hz}, \mathrm{H} 21), 8.08(1 \mathrm{H}, \mathrm{dd}, J=9.8,2.3 \mathrm{~Hz}, \mathrm{H} 22) .{ }^{13} \mathrm{C}\left\{{ }^{1} \mathrm{H}\right\}$ NMR (100 MHz, DMSO-d6) $\delta$ 16.9, 20.9, 21.0, 23.9, 26.5, 27.6, 29.6, 33.2, 34.8, 35.0, 35.7, 39.6 (deduced from HMQC), 41.5, 42.8, 48.8, 57.6, 64.7, 70.6, 83.3, 111.0, 118.8, 150.3, 151.5, 161.7. HRMS (ESI-TOF) $[\mathrm{M}+\mathrm{Na}]^{+} \mathrm{m} / \mathrm{z}$ : Calcd for $\mathrm{C}_{24} \mathrm{H}_{34} \mathrm{O}_{5} \mathrm{Na} 425.2298$; Found 425.2293.

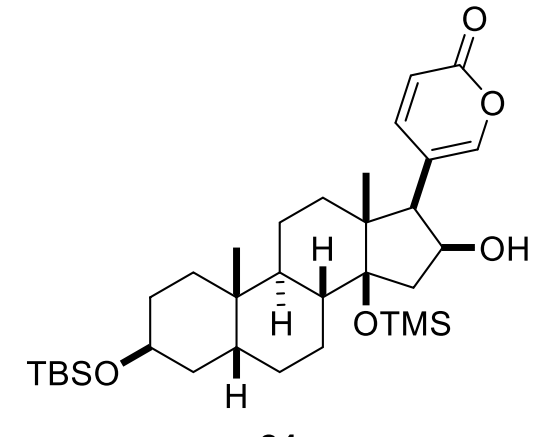

24

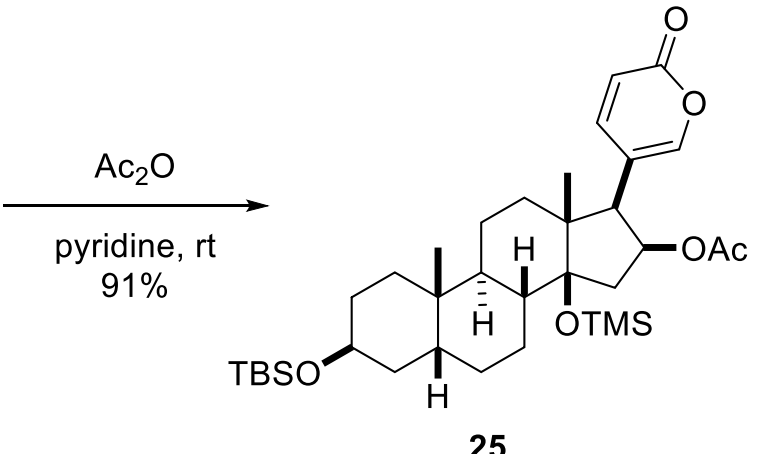

25

Acetate 25. $\quad \mathrm{Ac}_{2} \mathrm{O}(0.20 \mathrm{~mL}, 2.1 \mathrm{mmol})$ was added to a solution of alcohol $24(157 \mathrm{mg}, 267$ $\mu \mathrm{mol})$ in pyridine $(2.7 \mathrm{~mL})$ at room temperature. After the reaction mixture was stirred at room temperature for $18 \mathrm{~h}$, saturated aqueous $\mathrm{NH}_{4} \mathrm{Cl}(30 \mathrm{~mL})$ was added to the mixture. The resultant mixture was extracted with EtOAc $(30 \mathrm{~mL}$ x3). The combined organic layers were washed with brine $(30 \mathrm{~mL})$, dried over $\mathrm{Na}_{2} \mathrm{SO}_{4}$, filtered, and concentrated. The residue was purified by flash column chromatography on silica gel $(10 \mathrm{~g}$, hexane/EtOAc $=1 / 0$ to $10 / 1)$ to afford acetate $25(153 \mathrm{mg}, 242 \mu \mathrm{mol})$ in $91 \%$ yield: white solid. m.p. $160-163{ }^{\circ} \mathrm{C}$. $[\alpha]_{\mathrm{D}}{ }^{24}+9.5$ 
(c 0.76, $\mathrm{CHCl}_{3}$ ). IR (film) 3523, 3439, 2926, 2858, 1746, 1730, 1714, 1638, 1539, 1448, 1378, 1361, 1249, $1059 \mathrm{~cm}^{-1} .{ }^{1} \mathrm{H}$ NMR (400 MHz, $\left.\mathrm{CDCl}_{3}\right) \delta 0.01\left(6 \mathrm{H}, \mathrm{s}, \mathrm{CH}_{3}\right.$ of TBS), $0.18(9 \mathrm{H}, \mathrm{s}$, $\mathrm{CH}_{3}$ of TMS), 0.76 (3H, s, H18), 0.87 (9H, s, $t$-Bu of TBS), 0.90 (3H, s, H19), 1.07-1.87 (18H, $\mathrm{m}), 1.89$ (3H, s, CH3 CO), 2.45 (1H, dd, $J=15.6,8.7 \mathrm{~Hz}, \mathrm{H} 15 \mathrm{a}), 2.79$ (1H, d, J = 8.7 Hz, H17), $4.04(1 \mathrm{H}$, brs, H3), $5.46(1 \mathrm{H}, \mathrm{dd}, J=8.7,8.7 \mathrm{~Hz}, \mathrm{H} 16), 6.16(1 \mathrm{H}, \mathrm{d}, J=9.8 \mathrm{~Hz}, \mathrm{H} 23), 7.22(1 \mathrm{H}$, $\mathrm{d}, J=2.5 \mathrm{~Hz}, \mathrm{H} 21), 7.75(1 \mathrm{H}, \mathrm{dd}, J=9.8,2.5 \mathrm{~Hz}, \mathrm{H} 22) .{ }^{13} \mathrm{C}\left\{{ }^{1} \mathrm{H}\right\} \mathrm{NMR}\left(125 \mathrm{MHz}, \mathrm{CDCl}_{3}\right) \delta-$ 4.89 , -4.86, 3.1 18.06, 18.12, 20.5, 21.0, 23.4, 23.9, 25.8, 26.6, 28.5, 29.7, 34.4, 35.7, 35.8, 37.0, 41.0, 41.56, 41.65, 50.5, 57.2, 67.1, 74.0, 90.3, 112.7, 116.8, 149.5, 150.7, 162.0, 170.0 . HRMS (ESI-TOF) $[\mathrm{M}+\mathrm{Na}]^{+} \mathrm{m} / \mathrm{z}$ : Calcd for $\mathrm{C}_{35} \mathrm{H}_{58} \mathrm{O}_{6} \mathrm{Si}_{2} \mathrm{Na}$ 653.3670; Found 653.3643.
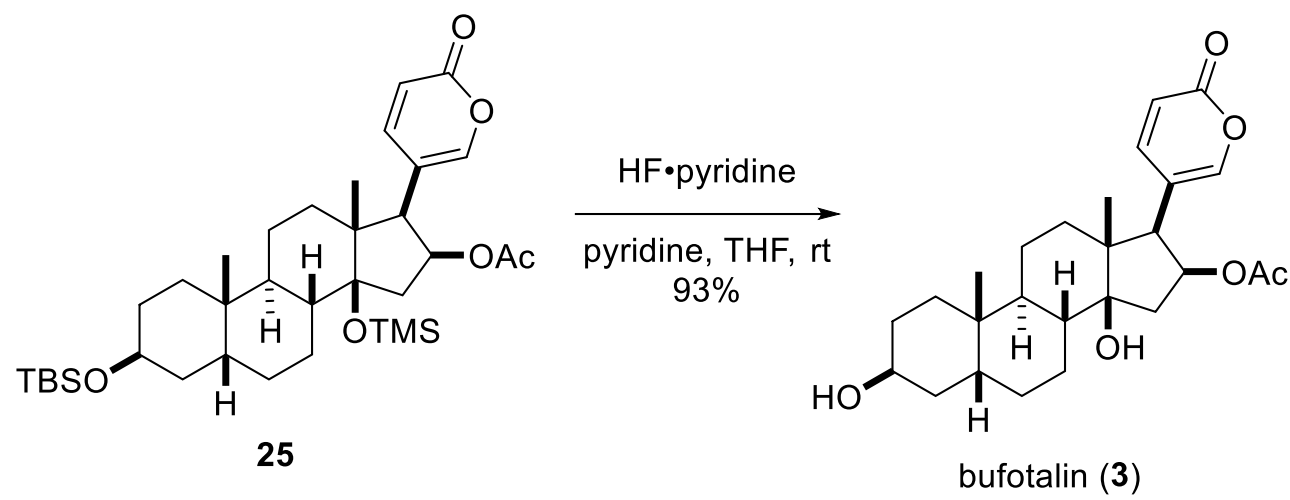

Bufotalin (3) [CAS: 471-95-4]. A mixture of HF (70 wt \% pyridine solution, $3.1 \mathrm{~mL}, 0.12$ mol) and pyridine $(3.1 \mathrm{~mL})$ was added to a solution of acetate $25(153 \mathrm{mg}, 242 \mu \mathrm{mol})$ in THF $(2.4 \mathrm{~mL})$ at room temperature. After the reaction mixture was stirred at room temperature for $124 \mathrm{~h}$, saturated aqueous $\mathrm{NaHCO}_{3}(50 \mathrm{~mL})$ was added to the mixture. The resultant mixture was extracted with EtOAc $(50 \mathrm{~mL} x 4)$. The combined organic layers were washed with brine $(20 \mathrm{~mL})$, dried over $\mathrm{Na}_{2} \mathrm{SO}_{4}$, filtered, and concentrated. The residue was purified by flash column chromatography on silica gel $(10 \mathrm{~g}$, hexane/EtOAc $=1 / 1$ to $1 / 3)$ to afford bufotalin $(3)$ $(100 \mathrm{mg}, 225 \mu \mathrm{mol})$ in $93 \%$ yield: colorless oil. $[\alpha]_{\mathrm{D}}{ }^{24}+5.3\left(c 1.28, \mathrm{CHCl}_{3}\right)$. IR (film) 3468 , 3057, 2935, 1713, 1633, 1537, 1448, 1378, 1250, 1136, 1092, 1037, $736 \mathrm{~cm}^{-1} .{ }^{1} \mathrm{H}$ NMR (500 $\left.\mathrm{MHz} \mathrm{CDCl}_{3}\right) \delta 0.78(3 \mathrm{H}, \mathrm{s}, \mathrm{H} 18), 0.94(3 \mathrm{H}, \mathrm{s}, \mathrm{H} 19), 1.15-1.32$ (5H, m, H1a, H6a, H7a, H11a, H12a), 1.37 (1H, d, J = 13.5 Hz, H4a), 1.42-1.61 (7H, m, H1b, H2, H8, H9, H11b, H12b), 1.751.78 (2H, m, H5, H7b), 1.81-1.92 (2H, m, H4b, H6b), $1.83(1 \mathrm{H}, \mathrm{d}, J=15.8 \mathrm{~Hz}, \mathrm{H} 15 \mathrm{a}), 1.86$ (3H, s, CH3 CO), $2.64(1 \mathrm{H}, \mathrm{dd}, J=15.8,8.9 \mathrm{~Hz}, \mathrm{H} 15 \mathrm{~b}), 2.86(1 \mathrm{H}, \mathrm{d}, J=8.9 \mathrm{~Hz}, \mathrm{H} 17), 4.14(1 \mathrm{H}$, brs, H3), $5.53(1 \mathrm{H}, \mathrm{dd}, J=8.9,8.9 \mathrm{~Hz}, \mathrm{H} 16), 6.19(1 \mathrm{H}, \mathrm{d}, J=9.8 \mathrm{~Hz}, \mathrm{H} 23), 7.24(1 \mathrm{H}, \mathrm{s}, \mathrm{H} 21)$, $8.03(1 \mathrm{H}, \mathrm{d}, J=9.8 \mathrm{~Hz}, \mathrm{H} 22) .{ }^{13} \mathrm{C}\left\{{ }^{1} \mathrm{H}\right\} \mathrm{NMR}\left(125 \mathrm{MHz}, \mathrm{CDCl}_{3}\right) \delta 16.4,20.9,21.0,21.1,23.7$, 26.3, 27.9, 29.5, 33.2, 35.3, 35.5, 35.9, 40.4, 40.8, 42.3, 49.4, 57.1, 66.7, 73.5, 84.4, 113.1, 116.8, 149.1, 150.9, 162.0, 170.0. HRMS (ESI-TOF) $[\mathrm{M}+\mathrm{Na}]^{+} \mathrm{m} / \mathrm{z}$ : Calcd for $\mathrm{C}_{26} \mathrm{H}_{36} \mathrm{O}{ }_{6} \mathrm{Na}$ 467.2404; Found 467.2396. 


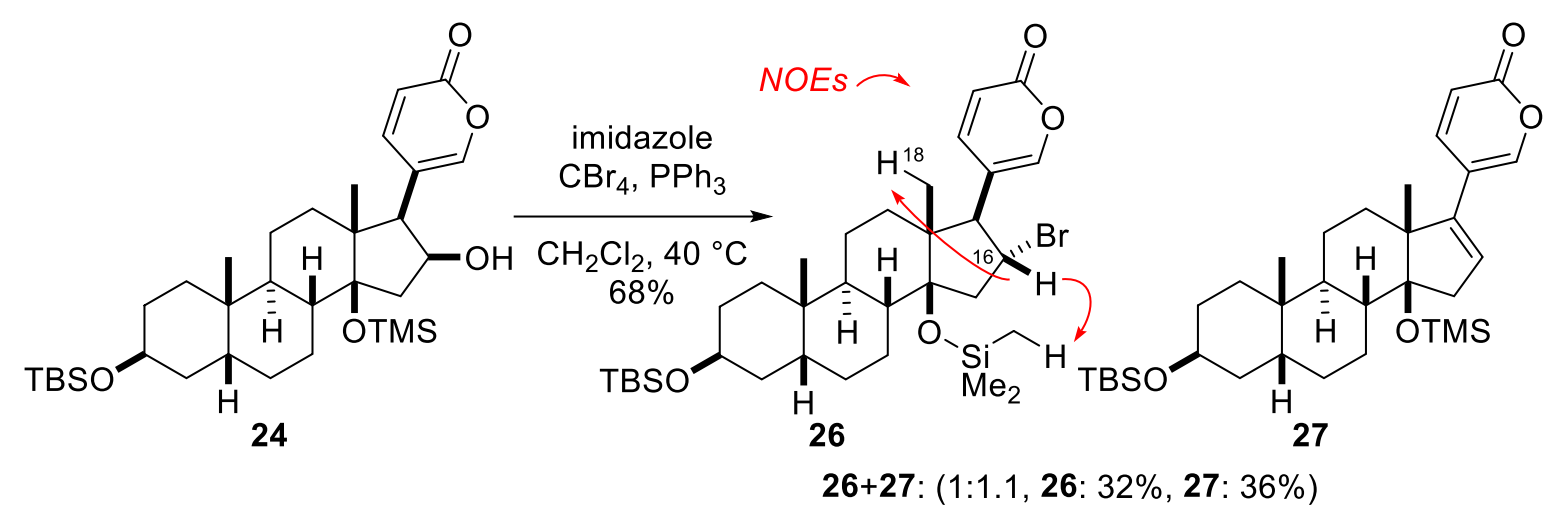

Bromide 26 and olefin 27. Imidazole (548 mg, $8.05 \mathrm{mmol}), \mathrm{CBr}_{4}(1.31 \mathrm{~g}, 3.95 \mathrm{mmol})$, and $\mathrm{PPh}_{3}(1.05 \mathrm{~g}, 4.00 \mathrm{mmol})$ were successively added to a solution of alcohol 24 (584 mg, 992 $\mu \mathrm{mol})$ in $\mathrm{CH}_{2} \mathrm{Cl}_{2}(5.0 \mathrm{~mL})$ at room temperature. After the reaction mixture was stirred at $40{ }^{\circ} \mathrm{C}$ for $21 \mathrm{~h}$, imidazole (533 mg, $7.83 \mathrm{mmol}), \mathrm{CBr}_{4}(1.35 \mathrm{~g}, 4.07 \mathrm{mmol})$, and $\mathrm{PPh}_{3}(1.06 \mathrm{~g}, 4.04$ mmol) were successively added to the mixture at room temperature. After the reaction mixture was stirred at $40{ }^{\circ} \mathrm{C}$ for $20 \mathrm{~h}$, imidazole (542 mg, $\left.7.96 \mathrm{mmol}\right), \mathrm{CBr}_{4}(1.31 \mathrm{~g}, 3.95 \mathrm{mmol})$, and $\mathrm{PPh}_{3}(1.04 \mathrm{~g}, 3.97 \mathrm{mmol})$ were successively added to the mixture at room temperature. After the reaction mixture was stirred at $40{ }^{\circ} \mathrm{C}$ for $9 \mathrm{~h}$, saturated aqueous $\mathrm{NaHCO}_{3}(50 \mathrm{~mL})$ was added to the mixture at room temperature. The resultant mixture was extracted with EtOAc $(50 \mathrm{~mL} \times 3)$. The combined organic layers were washed with brine $(30 \mathrm{~mL})$, dried over $\mathrm{Na}_{2} \mathrm{SO}_{4}$, filtered, and concentrated. The residue was purified by flash column chromatography on silica gel $(50 \mathrm{~g}$, hexane/EtOAc $=1 / 0$ to $50 / 1$ to $10 / 1)$ to afford a $1: 1.1$ mixture of bromide 26 and olefin 27 (411 mg, $317 \mu \mathrm{mol}$ for 26, $357 \mu \mathrm{mol}$ for 27 ) in $32 \%$ and $36 \%$ yields, respectively. For characterizations of $\mathbf{2 6}$ and $\mathbf{2 7}$, a small amount of the mixture was purified by GPC $\left(\mathrm{CHCl}_{3}\right)$. The C16-stereochemistry of $\mathbf{2 6}$ was determined by the NOE experiment.

26: colorless oil. $[\alpha]_{\mathrm{D}}{ }^{32}-31$ (c $\left.0.18, \mathrm{CHCl}_{3}\right)$. IR (film) 2931, 2858, 1747, 1641, 1543, 1466, 1380, 1253, 1065, $990 \mathrm{~cm}^{-1} .{ }^{1} \mathrm{H}$ NMR $\left(400 \mathrm{MHz}, \mathrm{CDCl}_{3}\right) \delta 0.02\left(6 \mathrm{H}, \mathrm{s}, \mathrm{CH}_{3}\right.$ of TBS), $0.20(9 \mathrm{H}$, s, $\mathrm{CH}_{3}$ of TMS), $0.70(3 \mathrm{H}, \mathrm{s}, \mathrm{H} 18), 0.88(9 \mathrm{H}, \mathrm{s}, t$-Bu of TBS), $0.90(3 \mathrm{H}, \mathrm{s}, \mathrm{H} 19), 1.04-1.16(1 \mathrm{H}$, $\mathrm{m}), 1.23-1.34(3 \mathrm{H}, \mathrm{m}), 1.39-1.42(3 \mathrm{H}, \mathrm{m}), 1.46-1.58(4 \mathrm{H}, \mathrm{m}), 1.67-1.73(3 \mathrm{H}, \mathrm{m}), 1.81-1.89(3 \mathrm{H}$, m), 2.27-2.41 (2H, m, H15), $2.73(1 \mathrm{H}, \mathrm{d}, J=8.0 \mathrm{~Hz}, \mathrm{H} 17), 4.07$ (1H, brs, H3), $4.24(1 \mathrm{H}, \mathrm{ddd}$, $J=11.2,8.0,8.0 \mathrm{~Hz}, \mathrm{H} 16), 6.29(1 \mathrm{H}, \mathrm{d}, J=9.6 \mathrm{~Hz}, \mathrm{H} 23), 7.27-7.28(1 \mathrm{H}, \mathrm{m}, \mathrm{H} 21), 7.41(1 \mathrm{H}$, $\mathrm{dd}, J=9.6,2.5 \mathrm{~Hz}, \mathrm{H} 22) .{ }^{13} \mathrm{C}\left\{{ }^{1} \mathrm{H}\right\} \mathrm{NMR}\left(100 \mathrm{MHz}, \mathrm{CDCl}_{3}\right) \delta-4.8,3.1,18.1,18.6,20.9,23.6$, 23.8, 25.8, 26.6, 28.5, 29.7, 34.3, 35.7, 35.8, 37.1, 40.6, 43.4, 45.1, 50.6, 52.1, 63.2, 67.1, 90.8, 115.6, 118.1, 145.0, 149.4, 161.7. HRMS (ESI-TOF) $[\mathrm{M}+\mathrm{Na}]^{+} \mathrm{m} / \mathrm{z}:$ Calcd for $\mathrm{C}_{33} \mathrm{H}_{55} \mathrm{BrO}_{4} \mathrm{Si}_{2} \mathrm{Na}$ 673.2714 and 675.2694; Found 673.2744 and 675.2686.

27: colorless oil. $[\alpha]_{\mathrm{D}}{ }^{23}+34\left(c 0.39, \mathrm{CHCl}_{3}\right)$. IR (film) 2933, 2887, 2858, 1747, 1447, 1249, $1225,1153,1088,1062 \mathrm{~cm}^{-1} .{ }^{1} \mathrm{H}$ NMR $\left(400 \mathrm{MHz}, \mathrm{C}_{6} \mathrm{D}_{6}\right) \delta 0.08\left(9 \mathrm{H}, \mathrm{s}, \mathrm{CH}_{3}\right.$ of TMS), $0.12(6 \mathrm{H}$, $\mathrm{s}, \mathrm{CH}_{3}$ of TBS), 0.82-0.99 (2H, m), $0.90(3 \mathrm{H}, \mathrm{s}, \mathrm{H} 19), 0.92(3 \mathrm{H}, \mathrm{s}, \mathrm{H} 18), 1.03(9 \mathrm{H}, \mathrm{s}, t$-Bu of TBS), 1.14-1.52 (9H, m), 1.65-2.00 (6H, m), $2.18(1 \mathrm{H}, \mathrm{dd}, J=17.4,3.2 \mathrm{~Hz}, \mathrm{H} 15 \mathrm{a}), 2.35(1 \mathrm{H}$, dd, $J=17.4,1.4 \mathrm{~Hz}, \mathrm{H} 15 \mathrm{~b}), 4.04$ (1H, brs, H3), 5.21 (1H, brs, H16), 5.97 (1H, dd, $J=9.8,0.9$ $\mathrm{Hz}, \mathrm{H} 23), 6.75(1 \mathrm{H}, \mathrm{dd}, J=9.8,2.5 \mathrm{~Hz}, \mathrm{H} 22), 7.07(1 \mathrm{H}, \mathrm{d}, J=2.5 \mathrm{~Hz}, \mathrm{H} 21) .{ }^{13} \mathrm{C}\left\{{ }^{1} \mathrm{H}\right\} \mathrm{NMR}$ 
$\left(100 \mathrm{MHz}, \mathrm{C}_{6} \mathrm{D}_{6}\right) \delta-4.7,-4.6,2.9,16.7,18.4,20.1,21.9,24.2,26.1,27.0,29.0,30.2,34.6,35.3$, 36.5, 36.6, 38.6, 39.0, 42.7, 53.0, 67.8, 90.7, 115.6, 116.4, 123.9, 143.1, 146.0, 147.9, 160.0. HRMS (ESI-TOF) $[\mathrm{M}+\mathrm{Na}]^{+} \mathrm{m} / \mathrm{z}$ : Calcd for $\mathrm{C}_{33} \mathrm{H}_{54} \mathrm{O}_{4} \mathrm{Si}_{2} \mathrm{Na}$ 593.3453; Found 593.3429.

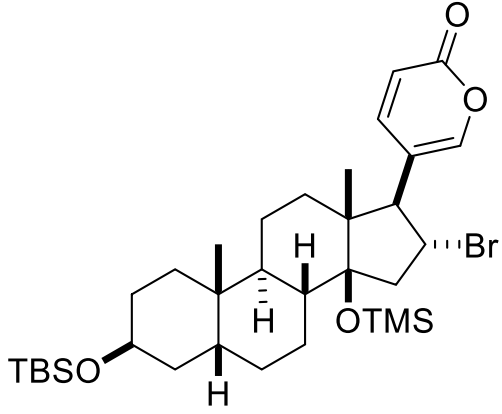

26

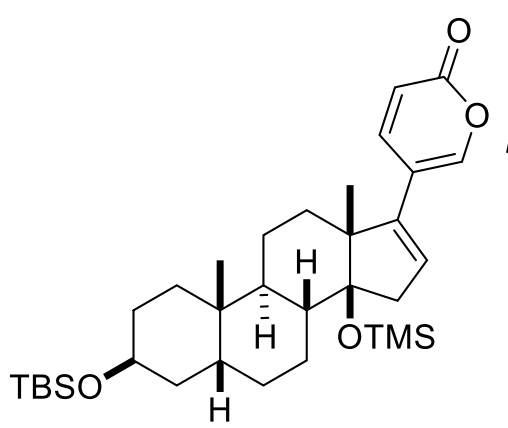

27

$(\mathbf{2 6}: \mathbf{2 7}=1: 1.1)$

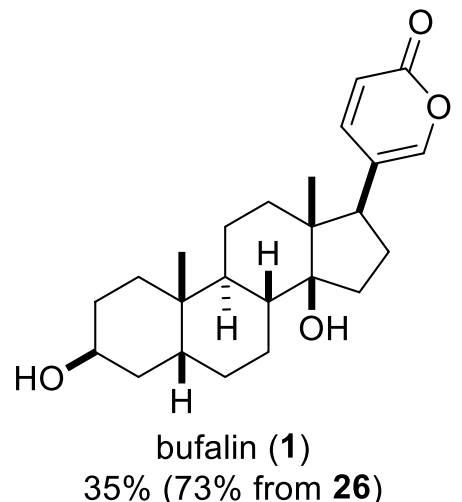

$35 \%(73 \%$ from 26$)$

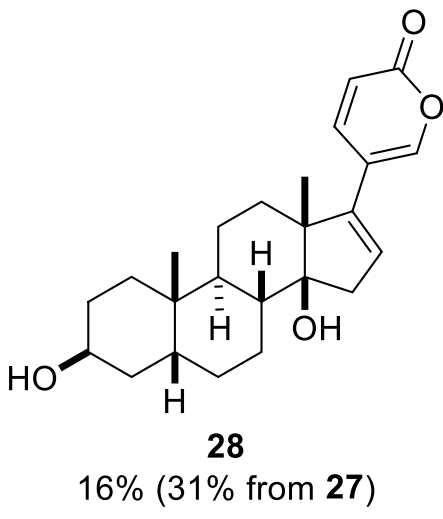

$n-\mathrm{Bu}_{3} \mathrm{SnH}, \mathrm{Et}_{3} \mathrm{~B}, \mathrm{O}_{2}$ hexane, $\mathrm{CH}_{2} \mathrm{Cl}_{2}$, rt;

$3 \mathrm{M} \mathrm{HCl}, \mathrm{MeOH}$

$\mathrm{rt}$

Bufalin (1) [CAS: 465-21-4] and diol 28. Et $_{3} \mathrm{~B}$ (1.00 M hexane solution, $1.15 \mathrm{~mL}, 1.15$ mmol) was added to a solution of a 1:1.1 mixture of bromide 26 and olefin 27 (297 $\mathrm{mg}, 230$ $\mu \mathrm{mol}$ for 26, $258 \mu \mathrm{mol}$ for 27$)$, and $n-\mathrm{Bu}_{3} \mathrm{SnH}(185 \mu \mathrm{L}, 0.687 \mathrm{mmol})$ in hexane $(8.1 \mathrm{~mL})$ and $\mathrm{CH}_{2} \mathrm{Cl}_{2}(8.1 \mathrm{~mL})$ at room temperature. The mixture was bubbled with $\mathrm{O}_{2}$ for $3 \mathrm{~min}$ at room temperature. After the reaction mixture was stirred under $\mathrm{O}_{2}$ atmosphere $(1 \mathrm{~atm})$ at room temperature for $2 \mathrm{~h}, \mathrm{Et}_{3} \mathrm{~B}(1.00 \mathrm{M}$ hexane solution, $1.15 \mathrm{~mL}, 1.15 \mathrm{mmol})$ and $n$ - $\mathrm{Bu}_{3} \mathrm{SnH}$ (185 $\mu \mathrm{mol}, 0.687 \mathrm{mmol}$ ) were successively added to the mixture. After the reaction mixture was stirred under $\mathrm{O}_{2}$ atmosphere ( $1 \mathrm{~atm}$ ) at room temperature for $2 \mathrm{~h}$, a mixture of $3 \mathrm{M}$ aqueous $\mathrm{HCl}$ $(2.5 \mathrm{~mL}, 7.5 \mathrm{mmol})$ and $\mathrm{MeOH}(30 \mathrm{~mL})$ was added to the mixture at room temperature. After the reaction mixture was stirred at room temperature for $20 \mathrm{~h}$, saturated aqueous $\mathrm{NaHCO}_{3}(50$ $\mathrm{mL})$ was added to the mixture. The resultant mixture was extracted with EtOAc $(50 \mathrm{~mL} \times 4)$. The combined organic layers were washed with brine $(30 \mathrm{~mL})$, dried over $\mathrm{Na}_{2} \mathrm{SO}_{4}$, filtered, and concentrated. The residue was purified by flash column chromatography (a column consecutively packed with silica gel $50 \mathrm{~g}$ and $10 \mathrm{wt} \% \mathrm{KF}$ contained silica gel $25 \mathrm{~g}$, hexane/EtOAc $=1 / 0$ to $3 / 2$ to $1 / 1$ to $1 / 3)$ to afford bufalin $(\mathbf{1})(65.2 \mathrm{mg}, 169 \mu \mathrm{mol})$ and diol 28 (30.6 mg, $79.6 \mu \mathrm{mol}$ ) in $35 \%$ and $16 \%$ yields, respectively.

1: colorless oil. $[\alpha]_{\mathrm{D}^{20}}+6.3\left(\right.$ c $\left.0.97, \mathrm{CHCl}_{3}\right)$. IR (film) 3456, 3059, 2933, 1711, 1538, 1450, $1255,1134 \mathrm{~cm}^{-1} .{ }^{1} \mathrm{H}$ NMR $\left(400 \mathrm{MHz}, \mathrm{CDCl}_{3}\right) \delta 0.70(3 \mathrm{H}, \mathrm{s}, \mathrm{H} 18), 0.95$ (3H, s, H19), 1.10-1.65 
(13H, m, H1, H2, H4a, H6a, H7a, H8, H9, H11, H12), 1.66-1.80 (4H, m, H5, H7b, H15a, H16a), 1.86-1.96 (2H, m, H4b, H6b), 2.02-2.11 (1H, m, H15b), 2.15-2.23 (1H, m, H16b), 2.46 (1H, $\mathrm{dd}, J=9.6,6.4 \mathrm{~Hz}, \mathrm{H} 17), 4.14(1 \mathrm{H}, \mathrm{brs}, \mathrm{H} 3), 6.26(1 \mathrm{H}, \mathrm{d}, J=9.8 \mathrm{~Hz}, \mathrm{H} 23), 7.23(1 \mathrm{H}, \mathrm{d}, J=$ $2.8 \mathrm{~Hz}, \mathrm{H} 21), 7.84(1 \mathrm{H}, \mathrm{dd}, J=9.8,2.8 \mathrm{~Hz}, \mathrm{H} 22) .{ }^{13} \mathrm{C}\left\{{ }^{1} \mathrm{H}\right\} \mathrm{NMR}\left(100 \mathrm{MHz}, \mathrm{CDCl}_{3}\right) \delta 16.5$, 21.4 (2C), 23.7, 26.5, 27.9, 28.7, 29.6, 32.7, 33.2 , 35.3, 35.6, 35.9, 40.9, 42.3, 48.3, 51.2, 66.8, 85.4, 115.3, 122.7, 146.8, 148.5, 162.4. HRMS (ESI-TOF) $[\mathrm{M}+\mathrm{Na}]^{+} \mathrm{m} / \mathrm{z}:$ Calcd for $\mathrm{C}_{24} \mathrm{H}_{34} \mathrm{O}_{4} \mathrm{Na}$ 409.2349; Found 409.2343.

28: white solid. m.p. $128-131^{\circ} \mathrm{C}$. $[\alpha]_{\mathrm{D}}{ }^{18}+73\left(c 0.12, \mathrm{CHCl}_{3}\right)$. IR (film) 3449, 3052, 2934, 2879 , $1719,1638,1532,1449,1379,1265,1226,1153,1125,1038 \mathrm{~cm}^{-1} .{ }^{1} \mathrm{H} \mathrm{NMR}\left(400 \mathrm{MHz}, \mathrm{CDCl}_{3}\right)$ $\delta 0.98(3 \mathrm{H}, \mathrm{s}, \mathrm{H} 18), 1.06-1.99(16 \mathrm{H}, \mathrm{m}), 1.14(3 \mathrm{H}, \mathrm{s}, \mathrm{H} 19), 2.26(1 \mathrm{H}, \mathrm{dd}, J=17.6,3.2 \mathrm{~Hz}$, H15a), 2.70 (1H, d, $J=17.6 \mathrm{~Hz}, \mathrm{H} 15 \mathrm{~b}), 4.14$ (1H, brs, H3), 5.77-5.79 (1H, m, H16), 6.33 (1H, $\mathrm{dd}, J=9.6,1.2 \mathrm{~Hz}, \mathrm{H} 23), 7.37-7.40(1 \mathrm{H}, \mathrm{m}, \mathrm{H} 22), 7.447-7.452(1 \mathrm{H}, \mathrm{m}, \mathrm{H} 21) .{ }^{13} \mathrm{C}\left\{{ }^{1} \mathrm{H}\right\} \mathrm{NMR}$ $\left(125 \mathrm{MHz}, \mathrm{CDCl}_{3}\right) \delta 16.2,19.8,21.1,23.9,26.2,27.9,29.6,33.3,35.2,35.93,35.95,38.6,39.8$, 40.7, 52.3, 66.8, 85.7, 116.0, 116.2, 125.0, 144.3, 145.1, 147.5, 161.2. HRMS (ESI-TOF) $[\mathrm{M}+\mathrm{Na}]^{+} \mathrm{m} / \mathrm{z}$ : Calcd for $\mathrm{C}_{24} \mathrm{H}_{32} \mathrm{O}_{4} \mathrm{Na}$ 407.2193; Found 407.2197.

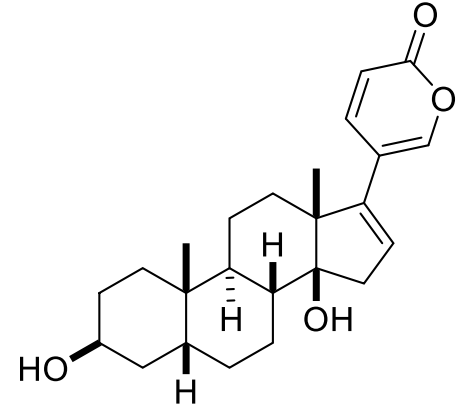

28

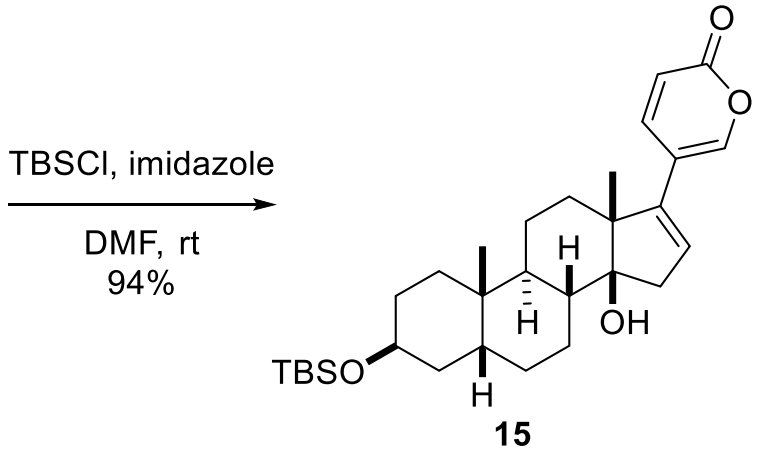

Alcohol 15 from diol 28 . TBSCl $(123 \mathrm{mg}, 816 \mu \mathrm{mol})$ was added to a solution of diol 28 (15.1 $\mathrm{mg}, 39.3 \mu \mathrm{mol})$ and imidazole $(104 \mathrm{mg}, 1.53 \mathrm{mmol})$ in DMF $(0.78 \mathrm{~mL})$ at room temperature. After the reaction mixture was stirred at room temperature for $16 \mathrm{~h}$, saturated aqueous $\mathrm{NaHCO}_{3}$ $(3 \mathrm{~mL})$ was added to the mixture. The resultant mixture was extracted with $\mathrm{Et}_{2} \mathrm{O}(3 \mathrm{~mL} \times 3)$. The combined organic layers were washed with brine $(3 \mathrm{~mL})$, dried over $\mathrm{Na}_{2} \mathrm{SO}_{4}$, filtered, and concentrated. The residue was purified by flash column chromatography on silica gel $(5 \mathrm{~g}$, hexane/EtOAc $=1 / 0$ to $1 / 1)$ to afford alcohol $15(18.4 \mathrm{mg}, 36.9 \mu \mathrm{mol})$ in $94 \%$ yield.

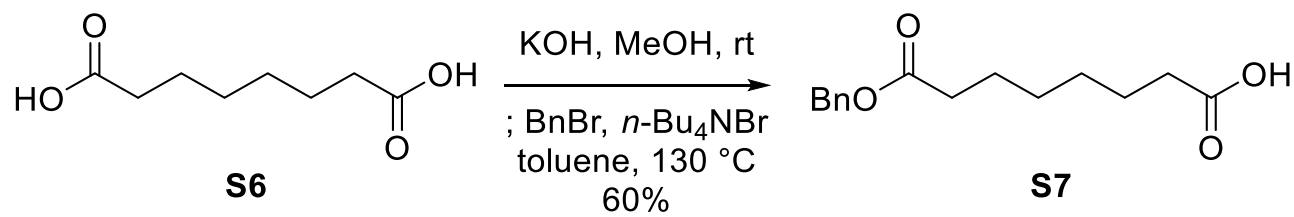

Carboxylic acid S7 [CAS: 15570-39-5]. ${ }^{\mathrm{S} 7} \mathrm{KOH}(79.2 \mathrm{mg}, 1.41 \mathrm{mmol})$ was added to a solution of suberic acid $(\mathbf{S 6}, 258 \mathrm{mg}, 1.48 \mathrm{mmol})$ in $\mathrm{MeOH}(11.4 \mathrm{~mL})$ at room temperature. After being stirred at room temperature for $20 \mathrm{~min}$, the reaction mixture was concentrated and dissolved in toluene $(2.5 \mathrm{~mL}) . \quad n-\mathrm{Bu} 4 \mathrm{NBr}(48.1 \mathrm{mg}, 0.149 \mathrm{mmol})$ and benzyl bromide $(\mathrm{BnBr}$, 
$0.20 \mathrm{~mL}, 1.7 \mathrm{mmol}$ ) were successively added to the solution at room temperature. After the reaction mixture was stirred at $130{ }^{\circ} \mathrm{C}$ for $13 \mathrm{~h}, 1 \mathrm{M}$ aqueous $\mathrm{HCl}(1.7 \mathrm{~mL}, 1.7 \mathrm{mmol})$ was added to the mixture at room temperature. The resultant mixture was extracted with $\mathrm{Et}_{2} \mathrm{O}(10$ $\mathrm{mL} \times 3)$. The combined organic layers were washed with $\mathrm{H}_{2} \mathrm{O}(10 \mathrm{~mL})$ and brine $(10 \mathrm{~mL})$, dried over $\mathrm{Na}_{2} \mathrm{SO}_{4}$, filtered, and concentrated. The residue was purified by flash column chromatography on silica gel $(10 \mathrm{~g}$, hexane/EtOAc $=1 / 0$ to $3 / 1)$ to afford carboxylic acid $\mathbf{S 7}$ (233 mg, $882 \mu \mathrm{mol}$ ) in 60\% yield: colorless oil. IR (film) 3033, 2937, 2862, 1735, 1706, 1458, 1414, 1240, $1171 \mathrm{~cm}^{-1} .{ }^{1} \mathrm{H}$ NMR (500 MHz, CDCl 3$) \delta 1.33-1.36$ (4H, m, H4', H5'), 1.61-1.68 (4H, m, H3', H6'), 2.33 (2H, t, $J=7.5$ Hz, H2'), 2.36 (2H, t, $J=7.5$ Hz, H7'), 5.11 (2H, s, $\left.\mathrm{PhCH}_{2}\right), 7.30-7.38\left(5 \mathrm{H}, \mathrm{m}\right.$, aromatic). ${ }^{13} \mathrm{C}\left\{{ }^{1} \mathrm{H}\right\} \mathrm{NMR}\left(125 \mathrm{MHz}, \mathrm{CDCl}_{3}\right) \delta 24.4,24.6,28.56$, 28.61, 33.9, 34.1, 66.1 128.1, 128.5, 136.0, 173.6, 179.9. HRMS (ESI-TOF) $[\mathrm{M}-\mathrm{H}+2 \mathrm{Na}]^{+} \mathrm{m} / \mathrm{z}$ : Calcd for $\mathrm{C}_{15} \mathrm{H}_{19} \mathrm{O}_{4} \mathrm{Na}_{2} 309.1073$; Found 309.1067.
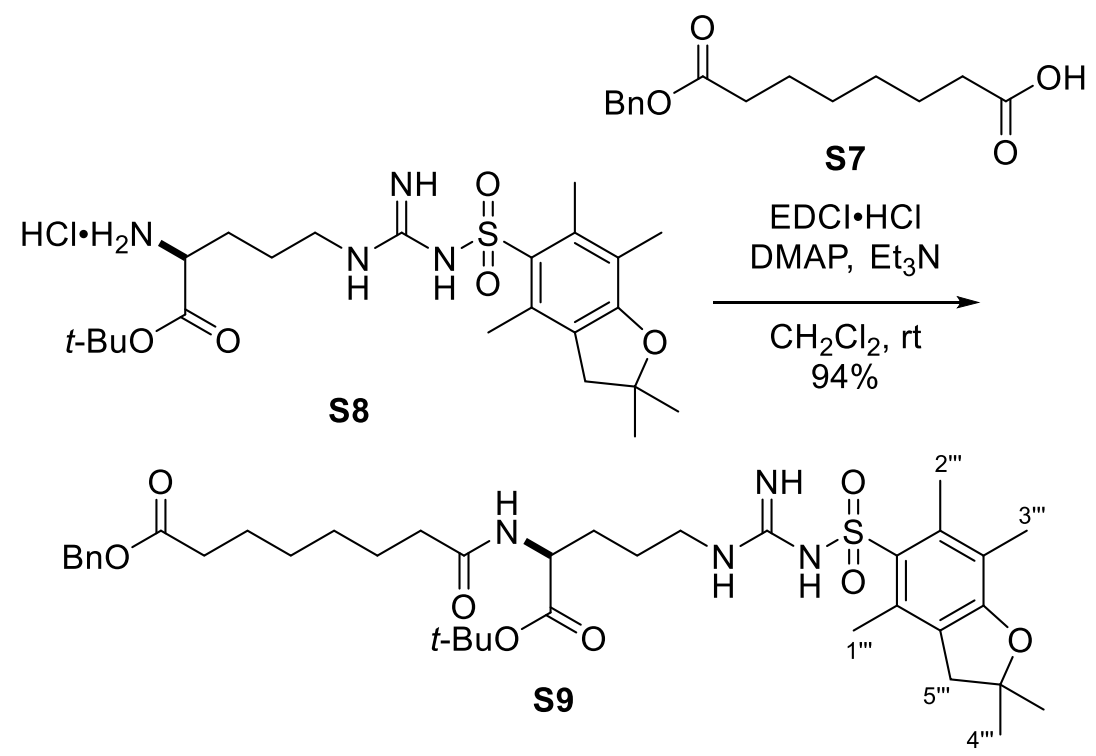

Benzyl ester S9. DMAP (14.9 $\mathrm{mg}, \quad 122 \mu \mathrm{mol}), \quad$ 1-ethyl-3-(3dimethylaminopropyl)carbodiimide hydrochloride (EDCI $\cdot \mathrm{HCl}, 87.6 \mathrm{mg}, 457 \mu \mathrm{mol}$ ), and $\mathrm{Et}_{3} \mathrm{~N}$ $(0.10 \mathrm{~mL}, 0.72 \mathrm{mmol})$ were successively added to a solution of amine hydrochloride $\mathbf{S 8}$ (159 $\mathrm{mg}, 306 \mu \mathrm{mol})$ and carboxylic acid $\mathbf{S 7}(121 \mathrm{mg}, 458 \mu \mathrm{mol})$ in $\mathrm{CH}_{2} \mathrm{Cl}_{2}(1.5 \mathrm{~mL})$ at room temperature. After the reaction mixture was stirred at room temperature for $19 \mathrm{~h}$, saturated aqueous $\mathrm{NaHCO}_{3}(5 \mathrm{~mL})$ was added to the mixture. The resultant mixture was extracted with EtOAc $(5 \mathrm{~mL} \mathrm{x} 4)$. The combined organic layers were washed with brine $(5 \mathrm{~mL})$, dried over $\mathrm{Na}_{2} \mathrm{SO}_{4}$, filtered, and concentrated. The residue was purified by flash column chromatography on silica gel $\left(10 \mathrm{~g}\right.$, hexane/EtOAc $=1 / 0$ to $2 / 1$ then $\left.\mathrm{CHCl}_{3} / \mathrm{MeOH}=20 / 1\right)$ to afford benzyl ester S9 $(211 \mathrm{mg}, 289 \mu \mathrm{mol})$ in $94 \%$ yield: colorless oil. $[\alpha]_{\mathrm{D}}{ }^{30}+5.4(c 0.31$, $\mathrm{CHCl}_{3}$ ). IR (film) 3441, 3337, 2933, 2863, 1734, 1622, 1551, 1455, 1370, 1253, 1157, 1108 $\mathrm{cm}^{-1} .{ }^{1} \mathrm{H}$ NMR $\left(500 \mathrm{MHz}, \mathrm{CDCl}_{3}\right) \delta 1.25-1.31(4 \mathrm{H}, \mathrm{m}), 1.44(9 \mathrm{H}, \mathrm{s}, t-\mathrm{Bu}), 1.45\left(6 \mathrm{H}, \mathrm{s}, \mathrm{H} 4{ }^{\prime \prime}, '\right)$, 1.57-1.65 (7H, m), 1.78-1.84 (1H, m), $2.08\left(3 \mathrm{H}, \mathrm{s}, \mathrm{H} 3{ }^{\prime \prime}\right.$ '), 2.17-2.20 (2H, m), $2.34(2 \mathrm{H}, \mathrm{t}, J=$ $7.5 \mathrm{~Hz}), 2.51$ (3H, s, H1','), 2.57 (3H, s, H2','), 2.94 (2H, s, H5','), 3.21 (1H, brs, H5' 'a), 3.30 (1H, brs, H5' 'b), 4.37-4.41 (1H, m, H2' '), 5.10 (2H, s, PhCH2), 6.29 (1H, brs, NH), 6.33 (1H, 
brs, $\mathrm{NH}), 6.39(1 \mathrm{H}, \mathrm{d}, J=7.8 \mathrm{~Hz}, \mathrm{NH}), 7.31-7.36\left(5 \mathrm{H}, \mathrm{m}\right.$, aromatic). ${ }^{13} \mathrm{C}\left\{{ }^{1} \mathrm{H}\right\} \mathrm{NMR}(100 \mathrm{MHz}$, $\left.\mathrm{CDCl}_{3}\right) \delta 12.4,17.9,19.2,24.7,25.1,25.3,27.9,28.55,28.65,28.7,34.1,36.2,40.6,43.2,66.1$, 82.4, 86.3, 117.4, 124.5, 128.11, 128.15, 128.5, 132.2, 132.9, 135.9, 138.3, 156.1, 158.6, 171.4, 173.6 (2C), two ${ }^{13} \mathrm{C}\left\{{ }^{1} \mathrm{H}\right\}$ peaks were not observed. HRMS (ESI-TOF) $[\mathrm{M}+\mathrm{Na}]^{+} \mathrm{m} / \mathrm{z}$ : Calcd for $\mathrm{C}_{38} \mathrm{H}_{56} \mathrm{~N}_{4} \mathrm{O}_{8} \mathrm{SNa}$ 751.3711; Found 751.3694.<smiles>CO[13C](=O)[O-]</smiles><smiles>[3H]c1c([3H])c(S(=O)(=O)NC(=N)NCCC[C@H](NC(=O)CCCCCCC(=O)O)C(=O)O[Na])c(F)c2c1OC(C)(C)C2</smiles>

Carboxylic acid 29a. A suspension of benzyl ester S9 (53.4 mg, $73.3 \mu \mathrm{mol})$ and $\mathrm{Pd} / \mathrm{C}$ (5 wt\% $\mathrm{Pd}$ on carbon, $10.7 \mathrm{mg}$ ) in $\mathrm{MeOH}(1.5 \mathrm{~mL})$ was stirred under $\mathrm{H}_{2}$ atmosphere $(1 \mathrm{~atm})$ at room temperature for $1 \mathrm{~h}$. The reaction mixture was then filtered through a pad of Celite with EtOAc $(10 \mathrm{~mL})$ and concentrated. The residue was purified by flash column chromatography on silica gel ( $5 \mathrm{~g}, \mathrm{CHCl}_{3} / \mathrm{MeOH}=1 / 0$ to $30 / 1$ to $\left.10 / 1\right)$ to afford carboxylic acid 29a $(41.5 \mathrm{mg}$, $65.0 \mu \mathrm{mol})$ in $89 \%$ yield: colorless oil. $[\alpha]_{\mathrm{D}^{28}}+7.6\left(c 0.91, \mathrm{CHCl}_{3}\right)$. IR (film) $3442,3337,2932$, $2863,1728,1629,1552,1456,1370,1252,1155,1105 \mathrm{~cm}^{-1} .{ }^{1} \mathrm{H}$ NMR (400 MHz, CD 3 OD) $\delta$ 1.34-1.37 (4H, m), $1.44(9 \mathrm{H}, \mathrm{s}, t-\mathrm{Bu}), 1.46(6 \mathrm{H}, \mathrm{s}, \mathrm{H} 4$ ', '), 1.53-1.67 (7H, m), 1.74-1.83 (1H, m, H3' 'a), 2.08 (3H, s, H3','), 2.23 (2H, t, $J=7.3 \mathrm{~Hz}), 2.26-2.30(2 \mathrm{H}, \mathrm{m}), 2.51$ (3H, s, H1','), 2.58 (3H, s, H2', '), 3.00 (2H, s, H5' ') ) 3.12-3.20 (2H, m, H5' '), 4.21 (1H, brs, H2' ), 8.16 (1H, $\mathrm{d}, J=7.8 \mathrm{~Hz}, \mathrm{NH}) .{ }^{13} \mathrm{C}\left\{{ }^{1} \mathrm{H}\right\} \mathrm{NMR}\left(125 \mathrm{MHz}, \mathrm{CD}_{3} \mathrm{OD}\right) \delta 12.5,18.4,19.6,25.91,25.94,26.8$, 27.1, 28.3, 28.7, 29.8, 29.9, 34.9, 36.6, 41.5, 44.0, 54.2, 82.7, 87.7, 118.4, 126.0, 133.5, 134.4, 139.4, 158.1, 159.9, 172.8, 176.3, 177.6. HRMS (ESI-TOF) $[\mathrm{M}+\mathrm{Na}]^{+} \mathrm{m} / \mathrm{z}:$ Calcd for $\mathrm{C}_{31} \mathrm{H}_{50} \mathrm{~N}_{4} \mathrm{O}_{8} \mathrm{SNa}$ 661.3242; Found 661.3260. 
<smiles>CC(=O)OC1CC(O)(C(C)(C)C)C2CCC3(C)C4CCC(OC(=O)CCCCCCC(=O)NC(CCCNC(=N)NS(=O)(=O)c5c(C)c(C)c6c(c5C)CC(C)(C)O6)C(=O)OC(C)(C)C)CC4(C)C3CCC2(C)C1c1ccc(=O)oc1</smiles>

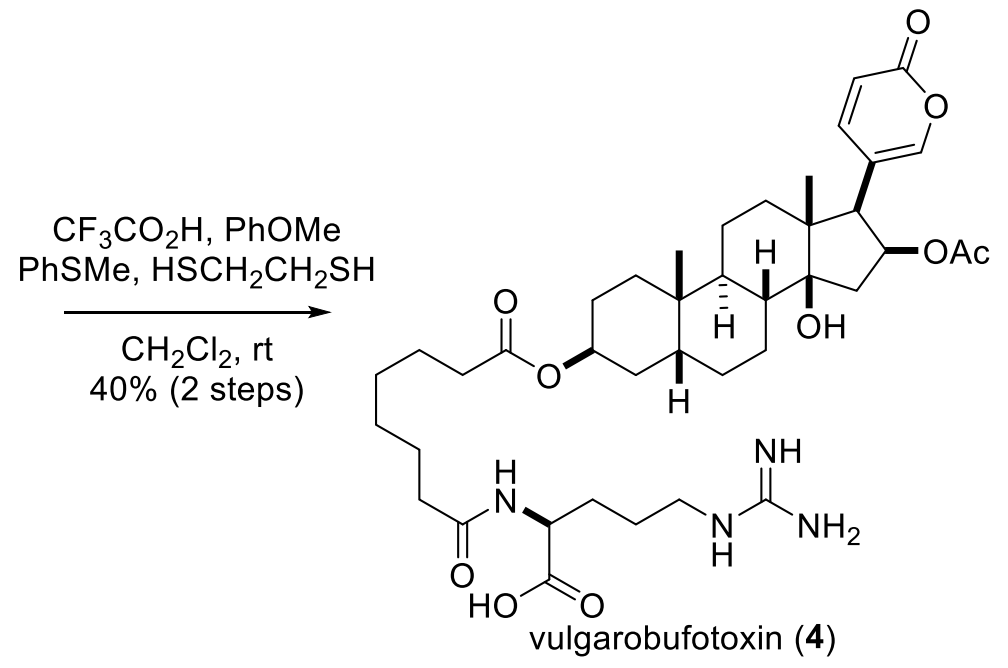

Vulgarobufotoxin (4) [CAS: 464-81-3]. Carboxylic acid 29a (21.7 mg, $34.0 \mu \mathrm{mol})$, DMAP (1.0 mg, $8.2 \mu \mathrm{mol}$ ), and $N, N$-diisopropylcarbodiimide (DIC, $5.0 \mu \mathrm{L}, 32 \mu \mathrm{mol}$ ) were added to a solution of bufotalin $(3)(12.6 \mathrm{mg}, 28.3 \mu \mathrm{mol})$ in $\mathrm{CH}_{2} \mathrm{Cl}_{2}(0.28 \mathrm{~mL})$ at room temperature. After the reaction mixture was stirred at room temperature for $15 \mathrm{~h}$, saturated aqueous $\mathrm{NaHCO}_{3}$ $(3 \mathrm{~mL})$ was added to the mixture. The resultant mixture was extracted with EtOAc $(3 \mathrm{~mL} \times 3)$. The combined organic layers were washed with brine $(3 \mathrm{~mL})$, dried over $\mathrm{Na}_{2} \mathrm{SO}_{4}$, filtered, and concentrated. The residue was purified by flash column chromatography on silica gel $(1 \mathrm{~g}$, hexane/EtOAc $=1 / 0$ to $1 / 1$ to $1 / 2$ then $\mathrm{CHCl}_{3} / \mathrm{MeOH}=20 / 1$ ) to afford the crude steroid 30a $(29.8 \mathrm{mg}$ ), which was used in the next reaction without further purification: colorless oil. HRMS (ESI-TOF) $[\mathrm{M}+\mathrm{Na}]^{+} \mathrm{m} / \mathrm{z}$ : Calcd for $\mathrm{C}_{57} \mathrm{H}_{84} \mathrm{~N}_{4} \mathrm{O}_{13} \mathrm{SNa}$ 1087.5648; Found 1087.5644.

A mixture of $\mathrm{CF}_{3} \mathrm{CO}_{2} \mathrm{H}(720 \mu \mathrm{L}), \mathrm{PhOMe}(16 \mu \mathrm{L}), \mathrm{PhSMe}(40 \mu \mathrm{L})$, and $\mathrm{HSCH}_{2} \mathrm{CH}_{2} \mathrm{SH}(24$ $\mu \mathrm{L})$ was added to a solution of a part of the above crude steroid $\mathbf{3 0 a}(16.6 \mathrm{mg})$ in $\mathrm{CH}_{2} \mathrm{Cl}_{2}(1.5$ $\mathrm{mL}$ ) at room temperature. After the reaction mixture was stirred at room temperature for 4.5 $\mathrm{h}$, toluene $(20 \mathrm{~mL})$ was added to the mixture and concentrated. The residue was purified by reversed-phase HPLC (column: Inertsil ODS-4 10 × $250 \mathrm{~mm}$, eluent A: $\mathrm{MeOH}+0.05 \%$ $\mathrm{CF}_{3} \mathrm{CO}_{2} \mathrm{H}$, eluent $\mathrm{B}: \mathrm{H}_{2} \mathrm{O}+0.05 \% \mathrm{CF}_{3} \mathrm{CO}_{2} \mathrm{H}, \mathrm{A} / \mathrm{B}=60 / 40$, flow rate: $2.0 \mathrm{~mL} / \mathrm{min}$, detection: $\mathrm{UV} 254 \mathrm{~nm}, t_{\mathrm{R}}=21.3 \mathrm{~min}$ ) to afford $\mathrm{CF}_{3} \mathrm{CO}_{2} \mathrm{H}$ salt of vulgarobufotoxin (4) $(5.47 \mathrm{mg}, 6.28$ 
$\mu \mathrm{mol}$ ) in $40 \%$ yield over 2 steps. Vulgarobufotoxin (4) was further purified by flash column chromatography on silica gel $\left(0.5 \mathrm{~g}, \mathrm{CH}_{2} \mathrm{Cl}_{2} / \mathrm{MeOH} / 28 \mathrm{wt} \%\right.$ aqueous $\left.\mathrm{NH}_{3}=28 / 8 / 1\right)$ to compare the NMR data with those prepared by the Pettit's route: ${ }^{\mathrm{S} 8}$ colorless oil. $[\alpha]_{\mathrm{D}}{ }^{28}+0.27$ (c 0.32 , MeOH). IR (film) 3332, 3179, 2931, 2862, 2360, 1711, 1538, 1450, 1379, 1246, 1201, 1135 $\mathrm{cm}^{-1} .{ }^{1} \mathrm{H}$ NMR $\left(500 \mathrm{MHz}, \mathrm{CD}_{3} \mathrm{OD}\right) \delta 0.78(3 \mathrm{H}, \mathrm{s}), 0.98(3 \mathrm{H}, \mathrm{s}), 1.17-1.46(13 \mathrm{H}, \mathrm{m}), 1.55-1.74$ $(13 \mathrm{H}, \mathrm{m}), 1.83-1.98(3 \mathrm{H}, \mathrm{m}), 1.85(3 \mathrm{H}, \mathrm{CH} 3 \mathrm{CO}), 2.01-2.06(1 \mathrm{H}, \mathrm{m}), 2.25(2 \mathrm{H}, \mathrm{t}, J=7.7 \mathrm{~Hz})$, $2.33(2 \mathrm{H}, \mathrm{t}, J=7.5 \mathrm{~Hz}), 2.71(1 \mathrm{H}, \mathrm{dd}, J=15.5,8.9 \mathrm{~Hz}, \mathrm{H} 15 \mathrm{a}), 2.97(1 \mathrm{H}, \mathrm{d}, J=8.9 \mathrm{~Hz}, \mathrm{H} 17)$, 3.16-3.26 (2H, m, H5' '), $4.32(1 \mathrm{H}, \mathrm{t}, J=5.8 \mathrm{~Hz}, \mathrm{H} 2$ ' '), $5.08(1 \mathrm{H}, \mathrm{s}, \mathrm{H} 3), 5.51(1 \mathrm{H}, \mathrm{dd}, J=8.9$, $8.9 \mathrm{~Hz}, \mathrm{H} 16), 6.21(1 \mathrm{H}, \mathrm{d}, J=10.0 \mathrm{~Hz}, \mathrm{H} 23), 7.44(1 \mathrm{H}, \mathrm{s}, \mathrm{H} 21), 8.25(1 \mathrm{H}, \mathrm{d}, J=10.0 \mathrm{~Hz}, \mathrm{H} 22)$. ${ }^{13} \mathrm{C}\left\{{ }^{1} \mathrm{H}\right\}$ NMR $\left(125 \mathrm{MHz}, \mathrm{CD}_{3} \mathrm{OD}\right) \delta 17.1,20.9,22.2,22.3,24.3,26.0,26.1,26.2,26.8,27.6$, $29.9,30.0,30.8,31.5,31.7,35.5,36.2$, 36.7, 37.1, 38.5, 41.1, 41.2, 42.0, 43.0, 50.8, 55.1 (deduced from HMQC), 58.2, 72.1, 75.7, 85.0, 113.2, 119.4, 152.1, 152.8, 158.6, 164.4, 171.9, 175.1, 175.6, 178.5 (deduced from HMBC). HRMS (ESI-TOF) $[\mathrm{M}+\mathrm{Na}]^{+} \mathrm{m} / \mathrm{z}$ : Calcd for $\mathrm{C}_{40} \mathrm{H}_{60} \mathrm{~N}_{4} \mathrm{O}_{10} \mathrm{Na} 779.4202$; Found 779.4214.
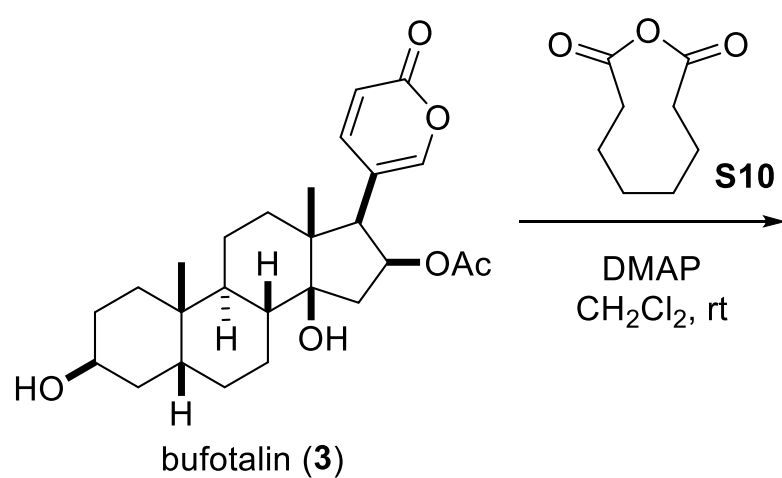

$N$-methylmorpholine MS 3A

THF, $-10{ }^{\circ} \mathrm{C}$;<smiles>N=C(N)NCCC[C@H](N)C(=O)O</smiles>

$1 \mathrm{M} \mathrm{HCl}, \mathrm{THF}, \mathrm{MeOH}$ $-10{ }^{\circ} \mathrm{C}$ to $\mathrm{rt}$ $58 \%$ (2 steps)

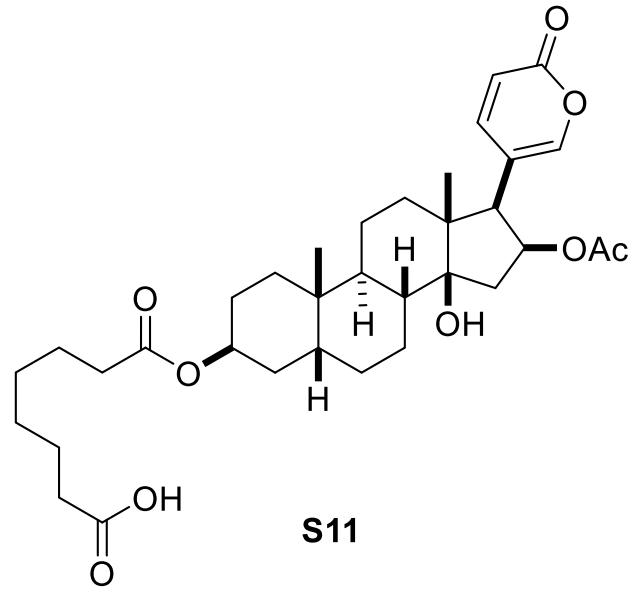

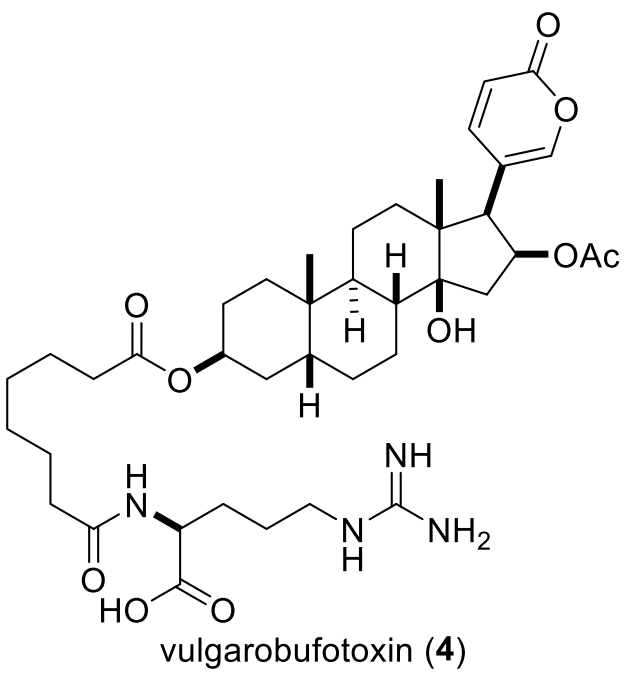

Vulgarobufotoxin (4) prepared by the Pettit's route. ${ }^{\mathrm{S} 8}$ A solution of suberic acid (S6, 1.04 $\mathrm{g}, 5.97 \mathrm{mmol})$ in $\mathrm{Ac}_{2} \mathrm{O}(2.1 \mathrm{~mL})$ was stirred at $150{ }^{\circ} \mathrm{C}$ for $2 \mathrm{~h}$. The reaction mixture was concentrated and azeotroped with toluene $(2 \mathrm{~mL} \times 3)$ to afford the crude acid anhydride $\mathbf{S 1 0}$ 
[CAS: 10521-06-9] ${ }^{\mathrm{S} 9}$ (995 mg) as white solid, which was used in the next reaction without further purification.

The above crude acid anhydride $\mathbf{S 1 0}(3.6 \mathrm{mg})$ and DMAP $(0.7 \mathrm{mg}, 6 \mu \mathrm{mol})$ were added to a solution of bufotalin $(3)(5.2 \mathrm{mg}, 12 \mu \mathrm{mol})$ in $\mathrm{CH}_{2} \mathrm{Cl}_{2}(0.10 \mathrm{~mL})$ at room temperature. After the reaction mixture was stirred at room temperature for $11 \mathrm{~h}$, saturated aqueous $\mathrm{NH}_{4} \mathrm{Cl}(3 \mathrm{~mL})$ was added to the mixture. The resultant mixture was extracted with EtOAc $(3 \mathrm{~mL} \mathrm{x} 4)$. The combined organic layers were washed with brine $(3 \mathrm{~mL})$, dried over $\mathrm{Na}_{2} \mathrm{SO}_{4}$, filtered, and concentrated. The residue was purified by PTLC $\left(\mathrm{CHCl}_{3} / \mathrm{MeOH}=20 / 1\right)$ to afford the crude steroid S11 (7.1 mg), which was used in the next reaction without further purification: colorless oil. HRMS (ESI-TOF) [M+Na] ${ }^{+} \mathrm{m} / \mathrm{z}$ : Calcd for $\mathrm{C}_{34} \mathrm{H}_{48} \mathrm{O}_{9} \mathrm{Na}$ 623.3191; Found 623.3193.

$N$-methyl morpholine $(6.0 \mu \mathrm{L}, 55 \mu \mathrm{mol})$ was added to a suspension of the above crude steroid S11 (7.1 mg) and activated molecular sieves 3A (MS 3A, $60.5 \mathrm{mg})$ in THF $(0.50 \mathrm{~mL})$ at $-10^{\circ} \mathrm{C}$. After the reaction mixture was stirred at $-10{ }^{\circ} \mathrm{C}$ for $10 \mathrm{~min}$, trichloroethyl chloroformate $(7.0$ $\mu \mathrm{L}, 52 \mu \mathrm{mol})$ was added to the mixture. After the reaction mixture was stirred at $-10{ }^{\circ} \mathrm{C}$ for $30 \mathrm{~min}$, a solution of L-arginine $(8.7 \mathrm{mg}, 50 \mu \mathrm{mol})$ in $1 \mathrm{M}$ aqueous $\mathrm{HCl}(15 \mu \mathrm{L}, 15 \mu \mathrm{mol})$ and $\mathrm{MeOH}(150 \mu \mathrm{L})$ was added to the mixture. After being stirred at room temperature for $2 \mathrm{~h}$, the reaction mixture was concentrated. The residue was purified by flash column chromatography on silica gel $\left(0.5 \mathrm{~g}, \mathrm{CH}_{2} \mathrm{Cl}_{2} / \mathrm{MeOH} / 28 \mathrm{wt} \%\right.$ aqueous $\left.\mathrm{NH}_{3}=28 / 8 / 1\right)$ to afford vulgarobufotoxin $(4)(5.2 \mathrm{mg}, 6.9 \mu \mathrm{mol})$ in $58 \%$ yield over 2 steps: colorless oil. $[\alpha]_{\mathrm{D}}{ }^{28}-1.2(c$ $0.26, \mathrm{MeOH})$. IR (film) 3386, 2932, 2861, 1712, 1537, 1378, 1246, 1201, $1134 \mathrm{~cm}^{-1} .{ }^{1} \mathrm{H}$ NMR (500 MHz, CD 3 OD) $\delta 0.78$ (3H, s, H18), 0.98 (3H, s, H19), 1.22-1.46 (13H, m), 1.55-1.74 (13H, $\mathrm{m}), 1.84-1.94(3 \mathrm{H}, \mathrm{m}), 1.85(3 \mathrm{H}, \mathrm{s}, \mathrm{CH} \mathrm{CO}), 2.01-2.06(1 \mathrm{H}, \mathrm{m}), 2.25(2 \mathrm{H}, \mathrm{t}, J=7.7 \mathrm{~Hz}), 2.33$ $(2 \mathrm{H}, \mathrm{t}, J=7.5 \mathrm{~Hz}), 2.71(1 \mathrm{H}, \mathrm{dd}, J=15.5,8.9 \mathrm{~Hz}, \mathrm{H} 15 \mathrm{a}), 2.97(1 \mathrm{H}, \mathrm{d}, J=8.9 \mathrm{~Hz}, \mathrm{H} 17), 3.17-$ 3.23 (2H, m, H5' '), 4.28-4.32 (1H, m, H2' ), 4.61 (1H, brs, OH), 5.08 (1H, brs, H3), $5.51(1 \mathrm{H}$, $\mathrm{dd}, J=8.9,8.9 \mathrm{~Hz}, \mathrm{H} 16), 6.21(1 \mathrm{H}, \mathrm{d}, J=10.1 \mathrm{~Hz}, \mathrm{H} 23), 7.44(1 \mathrm{H}, \mathrm{s}, \mathrm{H} 21), 8.11(1 \mathrm{H}, \mathrm{s}, \mathrm{NH})$, $8.25(1 \mathrm{H}, \mathrm{d}, J=10.1 \mathrm{~Hz}, \mathrm{H} 22) .{ }^{13} \mathrm{C}\left\{{ }^{1} \mathrm{H}\right\} \mathrm{NMR}\left(100 \mathrm{MHz}, \mathrm{CD}_{3} \mathrm{OD}\right) \delta 17.1,20.9,22.2,22.3$, 24.3, 25.9, 26.1 , 26.3, 26.8, 27.6, 29.9, 30.0, 30.5, 31.5, 31.7, 35.5, 36.2, 36.7, 36.9, 38.5, 41.1, 41.2, 41.9, 43.0, 50.8, 53.9, 58.2, 72.1, 75.7, 85.0, 113.2, 119.4, 152.1, 152.8, 158.6, 164.4, 171.9, 175.1, 175.9, 176.6. HRMS (ESI-TOF) $[\mathrm{M}+\mathrm{Na}]^{+} \mathrm{m} / \mathrm{z}$ : Calcd for $\mathrm{C}_{40} \mathrm{H}_{60} \mathrm{~N}_{4} \mathrm{O}_{10} \mathrm{Na}$ 779.4204; Found 779.4224. 


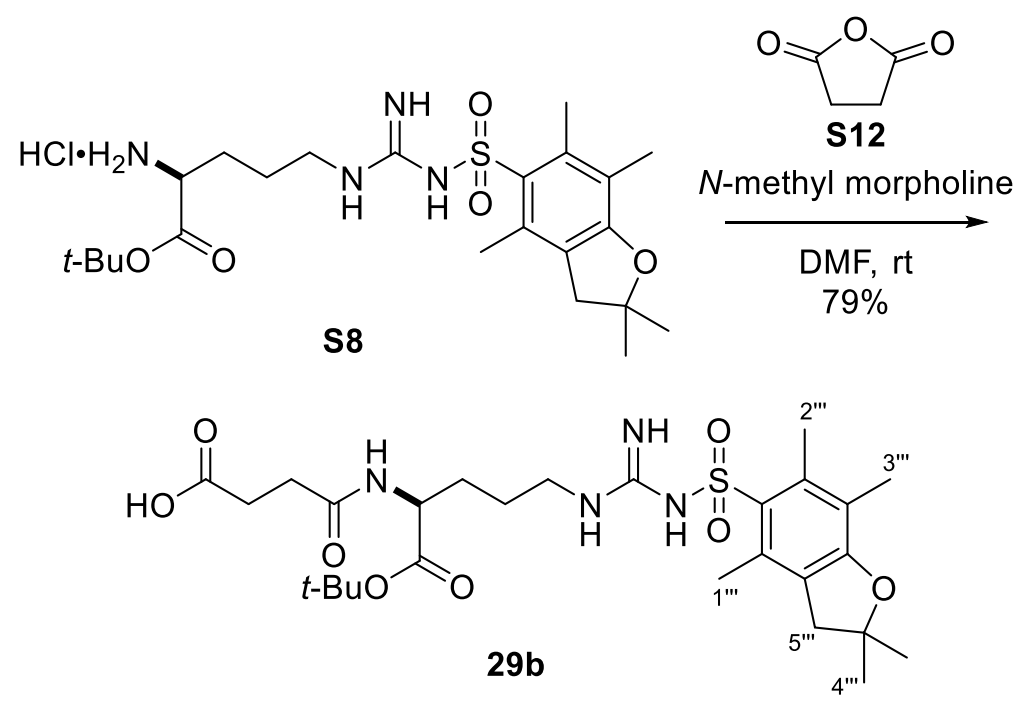

Carboxylic acid 29b. Acid anhydride S12 (10.4 mg, $104 \mu \mathrm{mol})$ and $N$-methyl morpholine $(23 \mu \mathrm{L}, 0.21 \mathrm{mmol})$ were successively added to a solution of amine hydrochloride $\mathbf{S 8}$ (54.2 $\mathrm{mg}$, $104 \mu \mathrm{mol})$ in DMF $(0.20 \mathrm{~mL})$ at room temperature. After the reaction mixture was stirred at room temperature for $4 \mathrm{~h}$, saturated aqueous $\mathrm{NH}_{4} \mathrm{Cl}(3 \mathrm{~mL})$ was added to the mixture. The resultant mixture was extracted with EtOAc $(3 \mathrm{~mL} \mathrm{x} 4)$. The combined organic layers were washed with brine $(3 \mathrm{~mL})$, dried over $\mathrm{Na}_{2} \mathrm{SO}_{4}$, filtered, and concentrated. The residue was purified by flash column chromatography on silica gel $\left(5 \mathrm{~g}, \mathrm{CHCl}_{3} / \mathrm{MeOH}=1 / 0\right.$ to $30 / 1$ to $\left.10 / 1\right)$ to afford carboxylic acid $29 \mathrm{~b}(47.8 \mathrm{mg}, 82.0 \mu \mathrm{mol})$ in $79 \%$ yield: colorless oil. $[\alpha]_{\mathrm{D}}{ }^{18}+16(c$ 1.2, $\mathrm{CHCl}_{3}$ ). IR (film) 3442, 3336, 2973, 2929, 2871, 1731, 1656, 1551, 1454, 1371, 1253, $1157,1105 \mathrm{~cm}^{-1} .{ }^{1} \mathrm{H}$ NMR $\left(500 \mathrm{MHz}, \mathrm{CDCl}_{3}\right) \delta 1.43(9 \mathrm{H}, \mathrm{s}, t-\mathrm{Bu}), 1.45\left(6 \mathrm{H}, \mathrm{s}, \mathrm{H} 4{ }^{\prime \prime \prime}\right), 1.54-$ 1.58 (2H, m, H4' '), 1.64-1.71 (1H, m, H3'’a), 1.82-1.88 (1H, m, H3' 'b), 2.08 (3H, s, H3','), 2.43-2.64 (3H, m), 2.48 (3H, s, H1 ', '), $2.54\left(3 \mathrm{H}, \mathrm{s}, \mathrm{H} 2{ }^{\prime \prime}{ }^{\prime}\right), 2.76-2.81(1 \mathrm{H}, \mathrm{m}), 2.94(2 \mathrm{H}, \mathrm{s}$, H5','), 3.18 (2H, brs, H5'), 4.44 (1H, brs, H2') 6.37 (2H, brs, NH), 6.88 (1H, brs, NHCO). ${ }^{13} \mathrm{C}\left\{{ }^{1} \mathrm{H}\right\} \mathrm{NMR}\left(125 \mathrm{MHz}, \mathrm{CDCl}_{3}\right) \delta 12.4,17.8,19.2,24.8,27.9,28.5,29.4,29.6,30.5,40.6$, 43.1, 52.6, 82.4, 86.4, 117.5, 124.6, 132.2 (2C), 138.3, 156.3, 158.7, 171.2, 172.7, 176.0 . HRMS (ESI-TOF) $[\mathrm{M}+\mathrm{Na}]^{+} \mathrm{m} / \mathrm{z}$ : Calcd for $\mathrm{C}_{27} \mathrm{H}_{42} \mathrm{~N}_{4} \mathrm{O}_{8} \mathrm{SNa}$ 605.2616; Found 605.2594. 


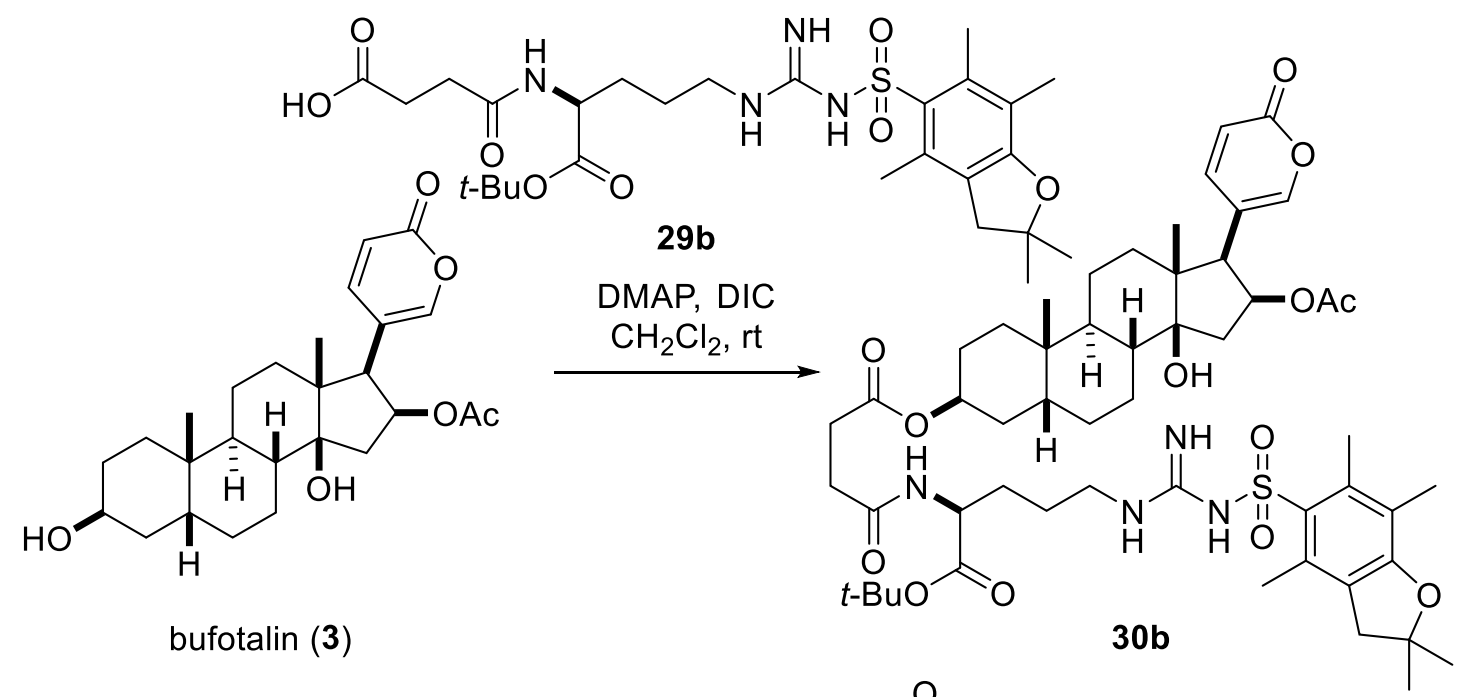

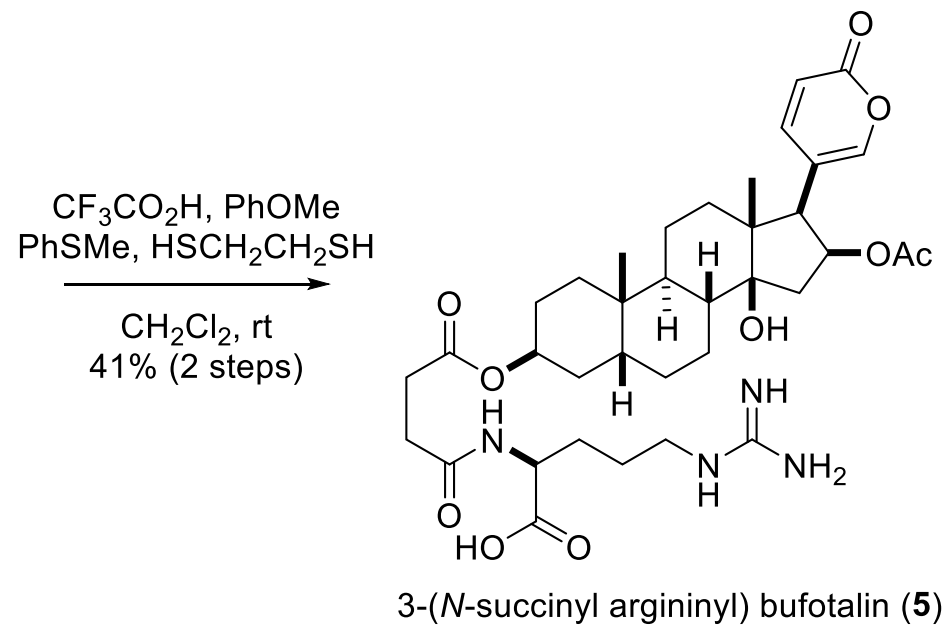

3-( $N$-succinyl argininyl) bufotalin (5) [CAS: 146996-65-8]. Carboxylic acid 29b $(8.92 \mathrm{mg}$, $15.3 \mu \mathrm{mol})$, DIC $(3.0 \mu \mathrm{L}, 19 \mu \mathrm{mol})$, and DMAP $(0.54 \mathrm{mg}, 4.4 \mu \mathrm{mol})$ were added to a solution of bufotalin (3) $(5.61 \mathrm{mg}, 12.6 \mu \mathrm{mol})$ in $\mathrm{CH}_{2} \mathrm{Cl}_{2}(0.25 \mathrm{~mL})$ at room temperature. After the reaction mixture was stirred at room temperature for $12 \mathrm{~h}$, saturated aqueous $\mathrm{NaHCO}_{3}(3 \mathrm{~mL})$ was added to the mixture. The resultant mixture was extracted with EtOAc $(3 \mathrm{~mL} \times 3)$. The combined organic layers were washed with brine $(3 \mathrm{~mL})$, dried over $\mathrm{Na}_{2} \mathrm{SO}_{4}$, filtered, and concentrated. The residue was purified by flash column chromatography on silica gel ( $0.5 \mathrm{~g}$, $\mathrm{CHCl}_{3} / \mathrm{MeOH}=1 / 0$ to 50/1) to afford the crude steroid 30b $(13.5 \mathrm{mg})$, which was used in the next reaction without further purification: colorless oil. HRMS (ESI-TOF) $[\mathrm{M}+\mathrm{Na}]^{+} \mathrm{m} / \mathrm{z}$ : Calcd for $\mathrm{C}_{53} \mathrm{H}_{76} \mathrm{~N}_{3} \mathrm{O}_{13} \mathrm{SNa}$ 1031.5022; Found 1031.5021.

A mixture of $\mathrm{CF}_{3} \mathrm{CO}_{2} \mathrm{H}(450 \mu \mathrm{L}), \mathrm{PhOMe}(10 \mu \mathrm{L}), \mathrm{PhSMe}(25 \mu \mathrm{L})$, and $\mathrm{HSCH}_{2} \mathrm{CH}_{2} \mathrm{SH}(15$ $\mu \mathrm{L}$ ) was added to a solution of the above crude steroid $30 \mathrm{~b}(13.5 \mathrm{mg})$ in $\mathrm{CH}_{2} \mathrm{Cl}_{2}(1.5 \mathrm{~mL})$ at room temperature. After the reaction mixture was stirred at room temperature for $4 \mathrm{~h}$, toluene $(20 \mathrm{~mL})$ was added to the mixture and concentrated. The residue was purified by reversedphase HPLC (column: Inertsil ODS-3 10 x $250 \mathrm{~mm}$, eluent A: $\mathrm{MeOH}+0.05 \% \mathrm{CF}_{3} \mathrm{CO}_{2} \mathrm{H}$, eluent $\mathrm{B}: \mathrm{H}_{2} \mathrm{O}+0.05 \% \mathrm{CF}_{3} \mathrm{CO}_{2} \mathrm{H}, \mathrm{A} / \mathrm{B}=40 / 60$, flow rate: $2.0 \mathrm{~mL} / \mathrm{min}$, detection: $\mathrm{UV} 254 \mathrm{~nm}, t_{\mathrm{R}}=$ $32.4 \mathrm{~min})$ to afford $\mathrm{CF}_{3} \mathrm{CO}_{2} \mathrm{H}$ salt of 3-( $N$-succinyl argininyl) bufotalin (5) $(4.21 \mathrm{mg}, 5.17 \mu \mathrm{mol})$ in $41 \%$ yield over 2 steps. 3 -( $N$-succinyl argininyl) bufotalin (5) was further purified by flash 
column chromatography on silica gel (first time: $0.5 \mathrm{~g}$, EtOAc/EtOH$/ \mathrm{H}_{2} \mathrm{O}=3 / 7 / 1$; second time: $\left.2.5 \mathrm{~g}, \mathrm{EtOAc} / \mathrm{EtOH} / \mathrm{H}_{2} \mathrm{O}=3 / 7 / 1\right)$ to compare the NMR data with those in the literature: $\mathrm{S}^{10}$ colorless oil. $[\alpha]_{\mathrm{D}}{ }^{25}-9.2$ (c 0.12, MeOH). IR (film) 3355, 2933, 2869, 1714, 1651, 1594, 1380, 1248, 1025, $854 \mathrm{~cm}^{-1} .{ }^{1} \mathrm{H}$ NMR (500 MHz, DMSO-d6) $\delta 0.65$ (3H, s, H18), 0.88 (3H, s, H19), 1.07-1.19 (2H, m), 1.23-1.78 (19H, m), 1.78 (3H, s, CH $3 \mathrm{CO}), 1.90-1.96(1 \mathrm{H}, \mathrm{m}), 2.38-2.46(4 \mathrm{H}$, m, H2', H3'), 2.67 (1H, dd, $J=16.9,8.6 \mathrm{~Hz}, \mathrm{H} 15 \mathrm{a}), 2.88$ (1H, d, $J=8.6 \mathrm{~Hz}, \mathrm{H} 17), 3.03$ (2H, brs, H5' '), $3.93(1 \mathrm{H}$, brs, H2' '), $4.41(1 \mathrm{H}, \mathrm{s}, \mathrm{OH}), 4.94(1 \mathrm{H}, \mathrm{s}, \mathrm{H} 3), 5.38(1 \mathrm{H}, \mathrm{dd}, J=8.6,8.6$ Hz, H16), $6.19(1 \mathrm{H}, \mathrm{d}, J=10.0 \mathrm{~Hz}, \mathrm{H} 23), 7.44-7.71(2 \mathrm{H}, \mathrm{m}, \mathrm{NH}), 7.51(1 \mathrm{H}, \mathrm{s}, \mathrm{H} 21), 7.62(1 \mathrm{H}$, brs, $\mathrm{NH}), 8.18(1 \mathrm{H}, \mathrm{d}, J=10.0 \mathrm{~Hz}, \mathrm{H} 22) .{ }^{13} \mathrm{C}\left\{{ }^{1} \mathrm{H}\right\} \mathrm{NMR}\left(125 \mathrm{MHz}, \mathrm{DMSO}-d_{6}\right) \delta 16.6,20.66$, 20.71, 20.8, 23.5, 24.3, 25.3, 26.1, 29.7, 29.8, 29.9, 30.2, 30.3, 34.6, 34.7, 36.6, 39.2 (deduced from HMQC), 40.0 (deduced from HMQC), 40.4, 41.3, 49.0, 53.6, 55.9, 70.0, 73.8, 82.6, 111.7, $117.3,150.1,151.4,157.3,161.2,169.5,169.7,171.8,175.9$. HRMS (ESI-TOF) $[\mathrm{M}+\mathrm{H}]^{+} \mathrm{m} / \mathrm{z}$ : Calcd for $\mathrm{C}_{36} \mathrm{H}_{53} \mathrm{~N}_{4} \mathrm{O}_{10}$ 701.3756; Found 701.3729. 
2. Comparison of ${ }^{1} \mathrm{H}$ and ${ }^{13} \mathrm{C}\left\{{ }^{1} \mathrm{H}\right\}$ NMR data of reported and synthetic $\mathbf{1}^{-5}$

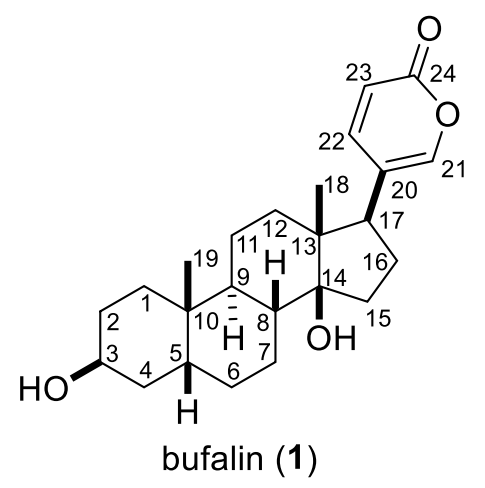

Table S1. ${ }^{1} \mathrm{H}$ NMR data of reported ${ }^{\mathrm{S} 11}$ and synthetic bufalin (1) in $\mathrm{CDCl}_{3}$

\begin{tabular}{|c|c|c|c|}
\hline \multirow{2}{*}{ No. } & reported $1(500 \mathrm{MHz})$ & synthetic $1(400 \mathrm{MHz})^{a}$ & \multirow[t]{2}{*}{$\Delta\left(\delta_{\text {reported }}-\delta_{\text {synthetic }}\right)$} \\
\hline & ${ }^{1} \mathrm{H}[\delta$, multi, $J(\mathrm{~Hz})]$ & ${ }^{1} \mathrm{H}[\delta$, multi, $J(\mathrm{~Hz})]$ & \\
\hline $1 \mathrm{a}$ & $1.42(\mathrm{~m})^{b}$ & $1.24(\mathrm{~m})$ & 0.18 \\
\hline $1 b$ & $1.49(\mathrm{~m})$ & $1.49(\mathrm{~m})$ & 0.00 \\
\hline $2 \mathrm{a}$ & $1.52(\mathrm{~m})$ & $1.52(\mathrm{~m})$ & 0.00 \\
\hline $2 b$ & $1.58(\mathrm{~m})$ & $1.59(\mathrm{~m})$ & -0.01 \\
\hline 3 & 4.14 (brs) & 4.14 (brs) & 0.00 \\
\hline $4 a$ & $1.35(\mathrm{~m})$ & $1.36(\mathrm{~m})$ & -0.01 \\
\hline $4 b$ & $1.88(\mathrm{~m})$ & $1.92(\mathrm{~m})$ & -0.04 \\
\hline 5 & $1.75(\mathrm{~m})$ & $1.76(\mathrm{~m})$ & -0.01 \\
\hline $6 a$ & $1.27(\mathrm{~m})$ & $1.27(\mathrm{~m})$ & 0.00 \\
\hline $6 b$ & $1.88(\mathrm{~m})$ & $1.91(\mathrm{~m})$ & -0.03 \\
\hline $7 \mathrm{a}$ & $1.18-1.72(\mathrm{~m})$ & $1.28(\mathrm{~m})$ & - \\
\hline $7 b$ & $1.18-1.72(\mathrm{~m})$ & $1.72(\mathrm{~m})$ & - \\
\hline 8 & $1.51(\mathrm{~m})$ & $1.53(\mathrm{~m})$ & -0.02 \\
\hline 9 & $1.61(\mathrm{~m})$ & $1.61(\mathrm{~m})$ & 0.00 \\
\hline $11 \mathrm{a}$ & $1.18-1.72(\mathrm{~m})$ & $1.17(\mathrm{~m})$ & - \\
\hline $11 b$ & $1.18-1.72(\mathrm{~m})$ & $1.42(\mathrm{~m})$ & - \\
\hline $12 \mathrm{a}$ & $1.39(\mathrm{~m})$ & $1.36(\mathrm{~m})$ & 0.03 \\
\hline $12 b$ & $1.49(\mathrm{~m})$ & $1.50(\mathrm{~m})$ & -0.01 \\
\hline $15 \mathrm{a}$ & $1.69(\mathrm{~m})$ & $1.69(\mathrm{~m})$ & 0.00 \\
\hline $15 b$ & $2.06(\mathrm{~m})$ & $2.04(\mathrm{~m})$ & 0.02 \\
\hline $16 \mathrm{a}$ & $1.72(\mathrm{~m})$ & $1.74(\mathrm{~m})$ & -0.02 \\
\hline $16 b$ & $2.18(\mathrm{~m})$ & $2.19(\mathrm{~m})$ & -0.01 \\
\hline 17 & $2.46(\mathrm{~m})$ & $2.46(\mathrm{dd}, 9.6,6.4)$ & 0.00 \\
\hline 18 & $0.70(\mathrm{~s})$ & $0.70(\mathrm{~s})$ & 0.00 \\
\hline 19 & $0.95(\mathrm{~s})$ & $0.95(\mathrm{~s})$ & 0.00 \\
\hline
\end{tabular}




\begin{tabular}{llll}
\hline 21 & $7.24(\mathrm{~d}, 2.6)$ & $7.23(\mathrm{~d}, 2.8)$ & 0.01 \\
22 & $7.85(\mathrm{dd}, 9.7,2.6)$ & $7.84(\mathrm{dd}, 9.8,2.8)$ & 0.01 \\
23 & $6.26(\mathrm{~d}, 9.7)$ & $6.26(\mathrm{~d}, 9.8)$ & 0.00 \\
\hline
\end{tabular}

${ }^{a}$ Chemical shifts were determined by using ${ }^{1} \mathrm{H}-{ }^{13} \mathrm{C}$ HMQC spectra. ${ }^{b}$ The $\delta$ value of this peak could be misreported.

Table S2. ${ }^{13} \mathrm{C}\left\{{ }^{1} \mathrm{H}\right\}$ NMR data of reported ${ }^{\mathrm{S} 11}$ and synthetic bufalin (1) in $\mathrm{CDCl}_{3}$

\begin{tabular}{|c|c|c|c|}
\hline \multirow{2}{*}{ No. } & reported $\mathbf{1}(125 \mathrm{MHz})$ & synthetic $\mathbf{1}(100 \mathrm{MHz})$ & \multirow[t]{2}{*}{$\Delta\left(\delta_{\text {reported }}-\delta_{\text {synthetic }}\right)$} \\
\hline & ${ }^{13} \mathrm{C}\left\{{ }^{1} \mathrm{H}\right\}(\delta)$ & ${ }^{13} \mathrm{C}\left\{{ }^{1} \mathrm{H}\right\}(\delta)$ & \\
\hline 1 & 29.62 & 29.59 & 0.03 \\
\hline 2 & 27.86 & 27.87 & -0.01 \\
\hline 3 & 66.85 & 66.81 & 0.04 \\
\hline 4 & 33.26 & 33.24 & 0.02 \\
\hline 5 & 35.96 & 35.94 & 0.02 \\
\hline 6 & 26.51 & 26.48 & 0.03 \\
\hline 7 & 21.40 & 21.37 & 0.03 \\
\hline 8 & 42.33 & 42.31 & 0.02 \\
\hline 9 & 35.37 & 35.35 & 0.02 \\
\hline 10 & 35.67 & 35.63 & 0.04 \\
\hline 11 & 21.40 & 21.37 & 0.03 \\
\hline 12 & 40.91 & 40.88 & 0.03 \\
\hline 13 & 48.36 & 48.32 & 0.04 \\
\hline 14 & 85.39 & 85.36 & 0.03 \\
\hline 15 & 32.71 & 32.70 & 0.01 \\
\hline 16 & 28.73 & 28.71 & 0.02 \\
\hline 17 & 51.21 & 51.18 & 0.03 \\
\hline 18 & 16.53 & 16.52 & 0.01 \\
\hline 19 & 23.73 & 23.72 & 0.01 \\
\hline 20 & 122.78 & 122.68 & 0.10 \\
\hline 21 & 148.52 & 148.46 & 0.06 \\
\hline 22 & 146.90 & 146.79 & 0.11 \\
\hline 23 & 115.27 & 115.28 & -0.01 \\
\hline 24 & 162.49 & 162.40 & 0.09 \\
\hline
\end{tabular}




\section{Supporting Information}

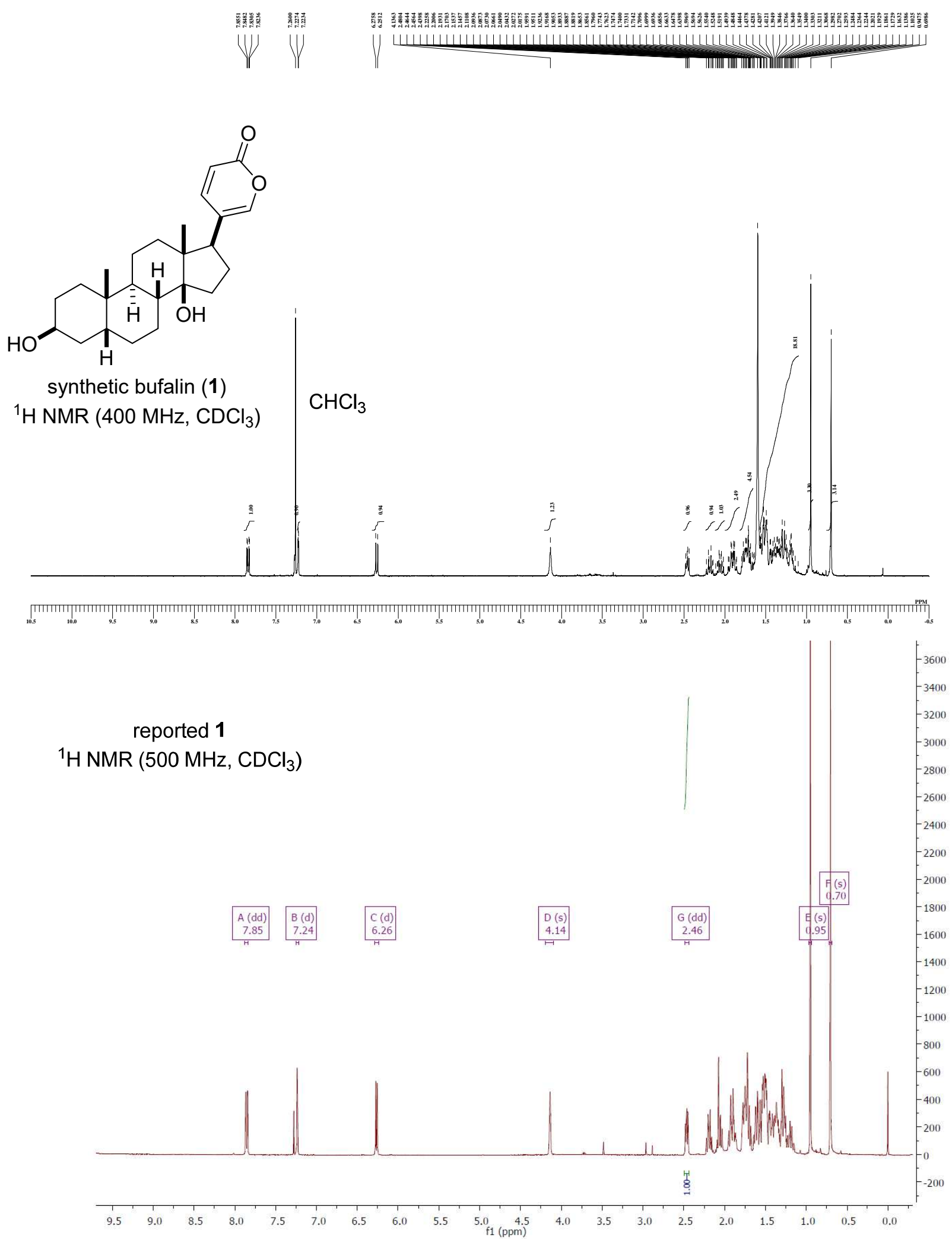

Figure S2. ${ }^{1} \mathrm{H}$ NMR spectra of synthetic $1(400 \mathrm{MHz})$ and reported $1(500 \mathrm{MHz})$ 


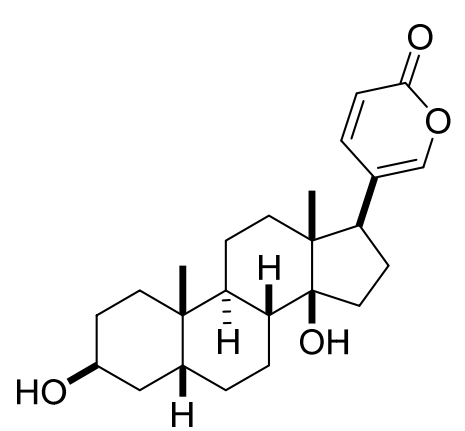

synthetic bufalin (1)

${ }^{13} \mathrm{C}\left\{{ }^{1} \mathrm{H}\right\}$ NMR $\left(100 \mathrm{MHz}, \mathrm{CDCl}_{3}\right)$

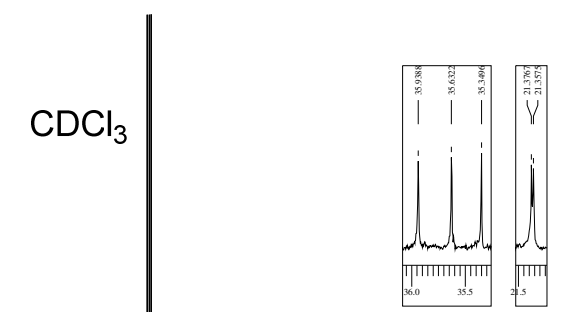

reported 1

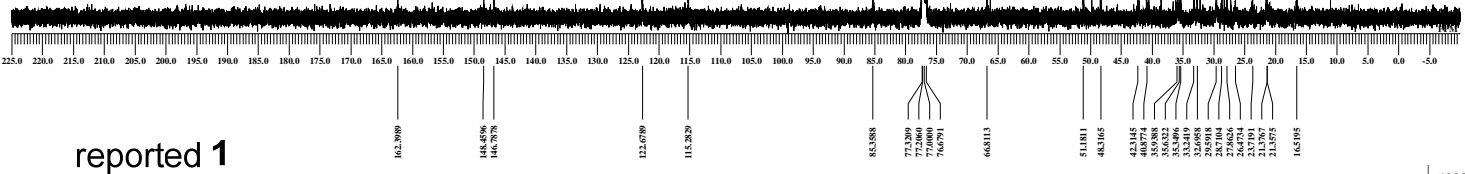

${ }^{13} \mathrm{C}\left\{{ }^{1} \mathrm{H}\right\}$ NMR $\left(125 \mathrm{MHz}, \mathrm{CDCl}_{3}\right)$

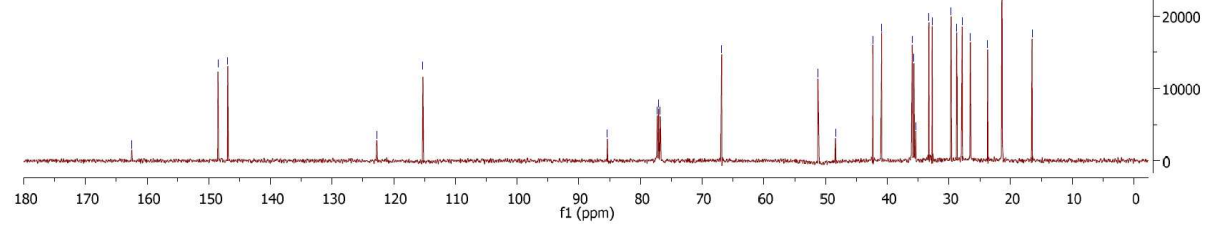

Figure S3. ${ }^{13} \mathrm{C}\left\{{ }^{1} \mathrm{H}\right\}$ NMR spectra of synthetic $1(100 \mathrm{MHz})$ and reported $1(125 \mathrm{MHz})$ 


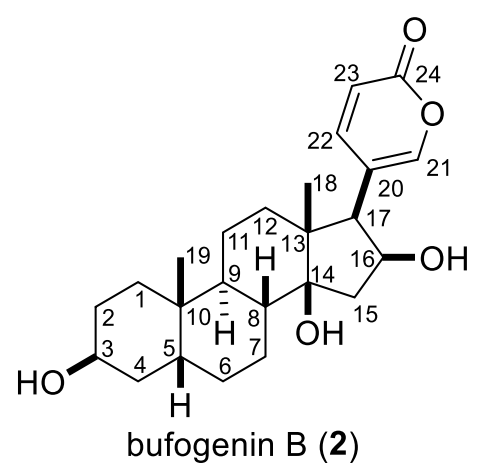

Table S3. ${ }^{1} \mathrm{H}$ NMR data of reported ${ }^{\mathrm{S} 12}$ and synthetic bufogenin B (2) in DMSO- $d_{6}$

\begin{tabular}{rllc}
\hline \multirow{2}{*}{ No. } & \multicolumn{1}{c}{ reported 2 $^{a}$} & synthetic 2 $(500 \mathrm{MHz})^{b}$ & \multirow{2}{*}{$\Delta\left(\delta_{\text {reported }}-\delta_{\text {synthetic }}\right)$} \\
\cline { 2 - 3 } 3 & ${ }^{1} \mathrm{H}[\delta$, multi, $J(\mathrm{~Hz})]$ & ${ }^{1} \mathrm{H}[\delta$, multi, $J(\mathrm{~Hz})]$ & \\
\hline 16 & $3.89(\mathrm{brs})$ & $3.89(\mathrm{brs})$ & 0.00 \\
17 & $4.43(\mathrm{~m})$ & $4.43(\mathrm{~m})$ & 0.00 \\
18 & $2.63(\mathrm{~d}, 8.1)$ & $2.63(\mathrm{~d}, 8.6)$ & 0.00 \\
19 & $0.64(\mathrm{~s})$ & $0.64(\mathrm{~s})$ & 0.00 \\
21 & $7.47(\mathrm{~d}, 1.5)$ & $0.85(\mathrm{~s})$ & 0.00 \\
22 & $8.08(\mathrm{dd}, 9.7,1.5)$ & $7.47(\mathrm{~d}, 2.3)$ & 0.00 \\
23 & $6.12(\mathrm{~d}, 9.7)$ & $8.08(\mathrm{dd}, 9.8,2.3)$ & 0.00 \\
\hline
\end{tabular}

${ }^{a}$ Internal standard was not indicated. ${ }^{b}$ Chemical shifts were determined by using ${ }^{1} \mathrm{H}-{ }^{13} \mathrm{C}$ HMQC spectra. 


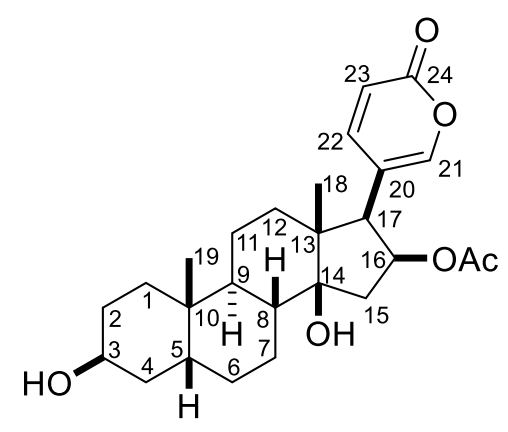

bufotalin (3)

Table S4. ${ }^{1} \mathrm{H}$ NMR data of reported ${ }^{\mathrm{S} 13}$ and synthetic bufotalin (3) in $\mathrm{CDCl}_{3}$

\begin{tabular}{|c|c|c|c|}
\hline \multirow{2}{*}{ No. } & reported $\mathbf{3}(500 \mathrm{MHz})^{a}$ & synthetic $3(500 \mathrm{MHz})^{b}$ & \multirow[t]{2}{*}{$\Delta\left(\delta_{\text {reported }}-\delta_{\text {synthetic }}\right)$} \\
\hline & ${ }^{1} \mathrm{H}[\delta]$ & ${ }^{1} \mathrm{H}[\delta$, multi, $J(\mathrm{~Hz})]$ & \\
\hline $1 \mathrm{a}$ & - & $1.25(\mathrm{~m})$ & - \\
\hline $1 b$ & 1.49 & $1.49(\mathrm{~m})$ & 0.00 \\
\hline $2 \mathrm{a}$ & 1.51 & $1.52(\mathrm{~m})$ & -0.01 \\
\hline $2 b$ & 1.56 & $1.58(\mathrm{~m})$ & -0.02 \\
\hline 3 & 4.14 & 4.14 (brs) & 0.00 \\
\hline $4 a$ & 1.37 & $1.37(\mathrm{~m})$ & 0.00 \\
\hline $4 \mathrm{~b}$ & 1.90 & $1.89(\mathrm{~m})$ & 0.01 \\
\hline 5 & 1.77 & 1.77 (brd, 12.6) & 0.00 \\
\hline $6 a$ & 1.29 & $1.29(\mathrm{~m})$ & 0.00 \\
\hline $6 b$ & 1.90 & $1.88(\mathrm{~m})$ & 0.02 \\
\hline $7 a$ & 1.21 & $1.20(\mathrm{~m})$ & 0.01 \\
\hline $7 b$ & 1.77 & $1.76(\mathrm{~m})$ & 0.01 \\
\hline 8 & 1.54 & $1.54(\mathrm{~m})$ & 0.00 \\
\hline 9 & 1.55 & $1.55(\mathrm{~m})$ & 0.00 \\
\hline $11 \mathrm{a}$ & 1.21 & $1.20(\mathrm{~m})$ & 0.01 \\
\hline $11 b$ & 1.44 & $1.44(\mathrm{~m})$ & 0.00 \\
\hline $12 \mathrm{a}$ & 1.28 & $1.29(\mathrm{~m})$ & -0.01 \\
\hline $12 b$ & 1.56 & $1.56(\mathrm{~m})$ & 0.00 \\
\hline $15 \mathrm{a}$ & 1.84 & $1.83(\mathrm{~d}, 15.8)$ & 0.01 \\
\hline $15 b$ & 2.64 & $2.64(\mathrm{dd}, 15.8,8.9)$ & 0.00 \\
\hline 16 & 5.54 & $5.53(\mathrm{dd}, 8.9,8.9)$ & 0.01 \\
\hline 17 & 2.89 & $2.86(\mathrm{~d}, 8.9)$ & 0.03 \\
\hline 18 & 0.78 & $0.78(\mathrm{~s})$ & 0.00 \\
\hline 19 & 0.98 & $0.94(\mathrm{~s})$ & 0.04 \\
\hline 21 & 7.25 & $7.24(\mathrm{~s})$ & 0.01 \\
\hline 22 & 8.03 & $8.03(\mathrm{~d}, 9.8)$ & 0.00 \\
\hline
\end{tabular}




\begin{tabular}{clll}
\hline 23 & 6.19 & $6.19(\mathrm{~d}, 9.8)$ & 0.00 \\
$\mathrm{Ac}$ & 1.87 & $1.86(\mathrm{~s})$ & 0.01 \\
\hline${ }^{\text {Internal standard }}$ & &
\end{tabular}

${ }^{a}$ Internal standard was not indicated. ${ }^{b}$ Chemical shifts were determined by using ${ }^{1} \mathrm{H}-{ }^{13} \mathrm{C}$ HMQC spectra.

Table S5. ${ }^{13} \mathrm{C}\left\{{ }^{1} \mathrm{H}\right\}$ NMR data of reported ${ }^{\mathrm{S} 13}$ and synthetic bufotalin (3) in $\mathrm{CDCl}_{3}$

\begin{tabular}{|c|c|c|c|}
\hline \multirow{2}{*}{ No. } & reported $\mathbf{3}(125 \mathrm{MHz})^{a}$ & synthetic $\mathbf{3}(125 \mathrm{MHz})$ & \multirow[t]{2}{*}{$\Delta\left(\delta_{\text {reported }}-\delta_{\text {synthetic }}\right)$} \\
\hline & ${ }^{13} \mathrm{C}\left\{{ }^{1} \mathrm{H}\right\}(\delta)$ & ${ }^{13} \mathrm{C}\left\{{ }^{1} \mathrm{H}\right\}(\delta)$ & \\
\hline 1 & 29.56 & 29.54 & 0.02 \\
\hline 2 & 27.91 & 27.88 & 0.03 \\
\hline 3 & 66.73 & 66.71 & 0.02 \\
\hline 4 & 33.27 & 33.23 & 0.04 \\
\hline 5 & 35.88 & 35.85 & 0.03 \\
\hline 6 & 26.37 & 26.34 & 0.03 \\
\hline 7 & 21.14 & 21.11 & 0.03 \\
\hline 8 & 42.32 & 42.27 & 0.05 \\
\hline 9 & 35.55 & 35.52 & 0.03 \\
\hline 10 & 35.29 & 35.26 & 0.03 \\
\hline 11 & 21.06 & 21.04 & 0.02 \\
\hline 12 & 40.42 & 40.38 & 0.04 \\
\hline 13 & 49.39 & 49.36 & 0.03 \\
\hline 14 & 84.41 & 84.39 & 0.02 \\
\hline 15 & 40.83 & 40.79 & 0.04 \\
\hline 16 & 73.57 & 73.54 & 0.03 \\
\hline 17 & 57.18 & 57.13 & 0.05 \\
\hline 18 & 16.44 & 16.44 & 0.00 \\
\hline 19 & 23.71 & 23.70 & 0.01 \\
\hline 20 & 116.83 & 116.84 & -0.01 \\
\hline 21 & 150.97 & 150.94 & 0.03 \\
\hline 22 & 149.13 & 149.13 & 0.00 \\
\hline 23 & 113.12 & 113.09 & 0.03 \\
\hline 24 & 161.93 & 161.95 & -0.02 \\
\hline $\mathrm{CO}$ of $\mathrm{Ac}$ & 170.02 & 170.02 & 0.00 \\
\hline $\mathrm{CH}_{3}$ of $\mathrm{Ac}$ & 20.94 & 20.94 & 0.00 \\
\hline
\end{tabular}

${ }^{a}$ Internal standard was not indicated. 


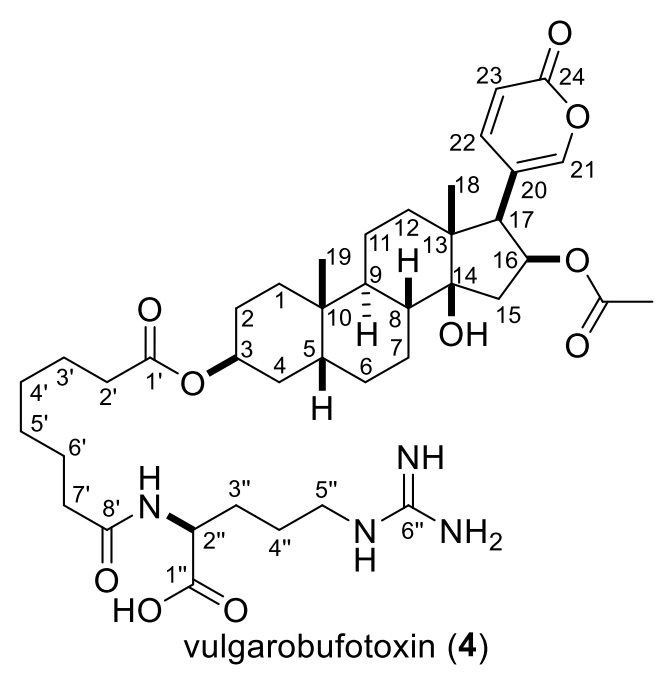

Table S6. ${ }^{1} \mathrm{H}$ NMR data of vulgarobufotoxin (4) prepared by our route and Pettit's route ${ }^{\mathrm{S} 8}$ in $\mathrm{CD}_{3} \mathrm{OD}$

\begin{tabular}{rllc}
\hline \multirow{2}{*}{ No. } & \multicolumn{1}{c}{$\begin{array}{c}\text { synthetic } 4(\mathrm{a})^{a} \\
(\text { our route, 500 MHz) }\end{array}$} & $\begin{array}{c}\text { synthetic } \mathbf{4}(\mathrm{b})^{a} \\
(\text { Pettit's route, 500 MHz) }\end{array}$ & $\begin{array}{c}\Delta\left(\delta_{\text {synthetic (a) }}\right. \\
\delta_{\text {synthetic (b) })}\end{array}$ \\
\cline { 2 - 3 } 3 & ${ }^{1} \mathrm{H}[\delta$, multi, $J(\mathrm{~Hz})]$ & ${ }^{1} \mathrm{H}[\delta$, multi, $J(\mathrm{~Hz})]$ & \\
\hline 16 & $5.08(\mathrm{~s})$ & $5.08(\mathrm{brs})$ & 0.00 \\
17 & $5.51(\mathrm{dd}, 8.9,8.9)$ & $5.51(\mathrm{dd}, 8.9,8.9)$ & 0.00 \\
18 & $2.97(\mathrm{~d}, 8.9)$ & $2.97(\mathrm{~d}, 8.9)$ & 0.00 \\
19 & $0.78(\mathrm{~s})$ & $0.78(\mathrm{~s})$ & 0.00 \\
21 & $7.94(\mathrm{~s})$ & $0.98(\mathrm{~s})$ & 0.00 \\
22 & $8.25(\mathrm{~d}, 10.0)$ & $7.44(\mathrm{~s})$ & 0.00 \\
23 & $6.21(\mathrm{~d}, 10.0)$ & $8.25(\mathrm{~d}, 10.1)$ & 0.00 \\
$2{ }^{2}$, & $4.32(\mathrm{t}, 5.8)$ & $6.21(\mathrm{~d}, 10.1)$ & 0.00 \\
Ac & $1.85(\mathrm{~s})$ & $4.30(\mathrm{~m})$ & 0.02 \\
\hline
\end{tabular}

${ }^{a}$ Chemical shifts were determined by using ${ }^{1} \mathrm{H}_{-}{ }^{13} \mathrm{C}$ HMQC spectra. 
Table S7. ${ }^{13} \mathrm{C}\left\{{ }^{1} \mathrm{H}\right\}$ NMR data of vulgarobufotoxin (4) prepared by our route and Pettit's route $\mathrm{S} 8$ in $\mathrm{CD}_{3} \mathrm{OD}$

\begin{tabular}{|c|c|c|}
\hline $\begin{array}{c}\text { synthetic } 4(\mathrm{a}) \\
\text { (our route, } 100 \mathrm{MHz} \text { ) }\end{array}$ & $\begin{array}{c}\text { synthetic } 4(\mathrm{~b}) \\
\text { (Pettit's route, } 125 \mathrm{MHz})\end{array}$ & $\Delta\left(\delta_{\text {synthetic (a) }}-\delta_{\text {synthetic (b) })}\right.$ \\
\hline${ }^{13} \mathrm{C}\left\{{ }^{1} \mathrm{H}\right\}(\delta)$ & ${ }^{13} \mathrm{C}\left\{{ }^{1} \mathrm{H}\right\}(\delta)$ & \\
\hline 17.12 & 17.13 & -0.01 \\
\hline 20.92 & 20.93 & -0.01 \\
\hline 22.21 & 22.21 & 0.00 \\
\hline 22.28 & 22.29 & -0.01 \\
\hline 24.31 & 24.31 & 0.00 \\
\hline 25.95 & 25.94 & 0.01 \\
\hline 26.12 & 26.12 & 0.00 \\
\hline 26.22 & 26.30 & -0.08 \\
\hline 26.84 & 26.82 & 0.02 \\
\hline 27.60 & 27.60 & 0.00 \\
\hline 29.90 & 29.90 & 0.00 \\
\hline 30.01 & 29.97 & 0.04 \\
\hline 30.76 & 30.47 & 0.29 \\
\hline 31.53 & 31.53 & 0.00 \\
\hline 31.69 & 31.69 & 0.00 \\
\hline 35.51 & 35.50 & 0.01 \\
\hline 36.25 & 36.25 & 0.00 \\
\hline 36.69 & 36.67 & 0.02 \\
\hline 37.12 & 36.92 & 0.20 \\
\hline 38.53 & 38.52 & 0.01 \\
\hline 41.12 & 41.11 & 0.01 \\
\hline 41.19 & 41.18 & 0.01 \\
\hline 42.02 & 41.93 & 0.09 \\
\hline 43.02 & 43.01 & 0.01 \\
\hline 50.76 & 50.75 & 0.01 \\
\hline 55.12 & 53.92 & 1.20 \\
\hline 58.20 & 58.18 & 0.02 \\
\hline 72.10 & 72.09 & 0.01 \\
\hline 75.71 & 75.70 & 0.01 \\
\hline 84.99 & 85.00 & -0.01 \\
\hline 113.15 & 113.16 & -0.01 \\
\hline 119.41 & 119.41 & 0.00 \\
\hline
\end{tabular}


Supporting Information

\begin{tabular}{lcc}
\hline 152.12 & 152.11 & 0.01 \\
152.81 & 152.80 & 0.01 \\
158.60 & 158.60 & 0.00 \\
164.44 & 164.42 & 0.02 \\
171.93 & 171.92 & 0.01 \\
175.06 & 175.06 & 0.00 \\
175.60 & 175.94 & -0.34 \\
178.50 & 176.61 & 1.89 \\
\hline
\end{tabular}




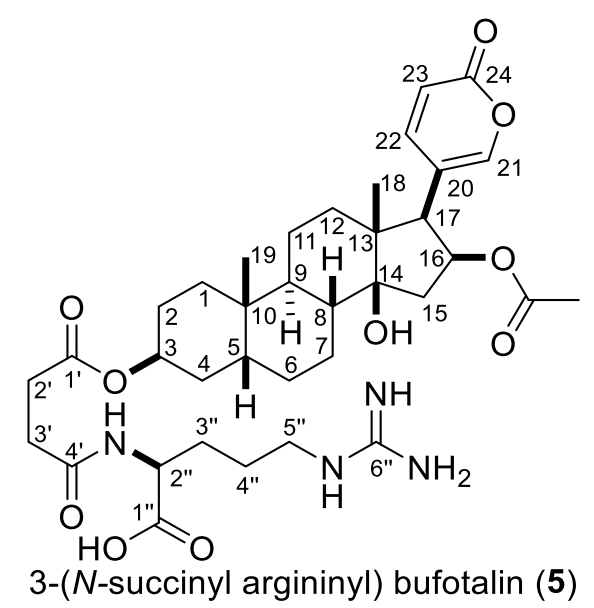

Table S8. ${ }^{1} \mathrm{H}$ NMR data of reported ${ }^{\mathrm{S} 10}$ and synthetic $3-(N$-succinyl argininyl) bufotalin (5) in DMSO- $d 6$

\begin{tabular}{|c|c|c|c|}
\hline \multirow{2}{*}{ No. } & reported $5(600 \mathrm{MHz})^{a, b}$ & synthetic $5(500 \mathrm{MHz})^{c}$ & \multirow[t]{2}{*}{$\Delta\left(\delta_{\text {reported }}-\delta_{\text {synthetic }}\right)$} \\
\hline & ${ }^{1} \mathrm{H}[\delta$, multi, $J(\mathrm{~Hz})]$ & ${ }^{1} \mathrm{H}[\delta$, multi, $J(\mathrm{~Hz})]$ & \\
\hline 3 & 4.95 (brs) & $4.94(\mathrm{~s})$ & 0.01 \\
\hline $15 \mathrm{a}$ & $2.67(\mathrm{~m})$ & $2.67(\mathrm{dd}, 16.9,8.6)$ & 0.00 \\
\hline 16 & $5.38(\mathrm{dd}, 8.4,8.4)$ & $5.38(\mathrm{dd}, 8.6,8.6)$ & 0.00 \\
\hline 17 & $2.88(\mathrm{~d}, 9.0)$ & $2.88(\mathrm{~d}, 8.6)$ & 0.00 \\
\hline 18 & $0.66(\mathrm{~s})$ & $0.65(\mathrm{~s})$ & 0.01 \\
\hline 19 & $0.89(\mathrm{~s})$ & $0.88(\mathrm{~s})$ & 0.01 \\
\hline 21 & $7.51(\mathrm{~s})$ & $7.51(\mathrm{~s})$ & 0.00 \\
\hline 22 & $8.18(\mathrm{~d}, 9.6)$ & $8.18(\mathrm{~d}, 10.0)$ & 0.00 \\
\hline 23 & $6.19(\mathrm{~d}, 9.6)$ & $6.19(\mathrm{~d}, 10.0)$ & 0.00 \\
\hline $2^{\prime \prime}$ & $3.92(\mathrm{~d}, 7.2)$ & 3.93 (brs) & -0.01 \\
\hline $5^{\prime \prime}$ & 3.03 (brs) & 3.03 (brs) & 0.00 \\
\hline $\mathrm{OH}$ & $4.38(\mathrm{~s})$ & $4.41(\mathrm{~s})$ & -0.03 \\
\hline
\end{tabular}

${ }^{a}$ Internal standard was not indicated. ${ }^{b}$ The ${ }^{1} \mathrm{H}$ NMR spectrum was provided by Professors HuiMin Gao and Zhi-Min Wang. ${ }^{c}$ Chemical shifts were assigned by using ${ }^{1} \mathrm{H}_{-}{ }^{13} \mathrm{C}$ HMQC spectra. 
Table S9. ${ }^{13} \mathrm{C}\left\{{ }^{1} \mathrm{H}\right\}$ NMR data of reported ${ }^{\mathrm{S} 10}$ and synthetic 3 -( $N$-succinyl argininyl) bufotalin (5) in DMSO-d6

\begin{tabular}{|c|c|c|}
\hline reported $\mathbf{5}(150 \mathrm{MHz})^{a, b}$ & synthetic $\mathbf{5}(125 \mathrm{MHz})$ & $\Delta\left(\delta_{\text {reported }}-\delta_{\text {synthetic }}\right)$ \\
\hline${ }^{13} \mathrm{C}\left\{{ }^{1} \mathrm{H}\right\}(\delta)$ & ${ }^{13} \mathrm{C}\left\{{ }^{1} \mathrm{H}\right\}(\delta)$ & \\
\hline 30.20 & 29.71 & 0.49 \\
\hline- & 29.80 & - \\
\hline 30.32 & 29.85 & 0.47 \\
\hline 30.67 & 30.17 & 0.50 \\
\hline- & 30.28 & - \\
\hline 35.02 & 34.55 & 0.47 \\
\hline 35.13 & 34.69 & 0.44 \\
\hline 37.02 & 36.58 & 0.44 \\
\hline- & 39.30 & - \\
\hline- & 40.00 & - \\
\hline 40.85 & 40.39 & 0.46 \\
\hline 41.76 & 41.30 & 0.46 \\
\hline 49.43 & 48.98 & 0.45 \\
\hline 54.13 & 53.61 & 0.52 \\
\hline 56.40 & 55.92 & 0.48 \\
\hline 70.41 & 69.96 & 0.45 \\
\hline 74.24 & 73.81 & 0.43 \\
\hline 83.04 & 82.59 & 0.45 \\
\hline 112.12 & 111.68 & 0.44 \\
\hline 117.72 & 117.29 & 0.43 \\
\hline 150.57 & 150.14 & 0.43 \\
\hline 151.89 & 151.44 & 0.45 \\
\hline 157.77 & 157.31 & 0.46 \\
\hline 161.59 & 161.18 & 0.41 \\
\hline 169.88 & 169.47 & 0.41 \\
\hline 170.03 & 169.70 & 0.33 \\
\hline 172.17 & 171.75 & 0.42 \\
\hline 176.24 & 175.91 & 0.33 \\
\hline
\end{tabular}

${ }^{a}$ Internal standard was not indicated. ${ }^{b}$ The ${ }^{13} \mathrm{C}\left\{{ }^{1} \mathrm{H}\right\}$ NMR spectrum was provided by Professors Hui-Min Gao and Zhi-Min Wang. 
3. X-ray structures and crystallographic data of 6 and 18
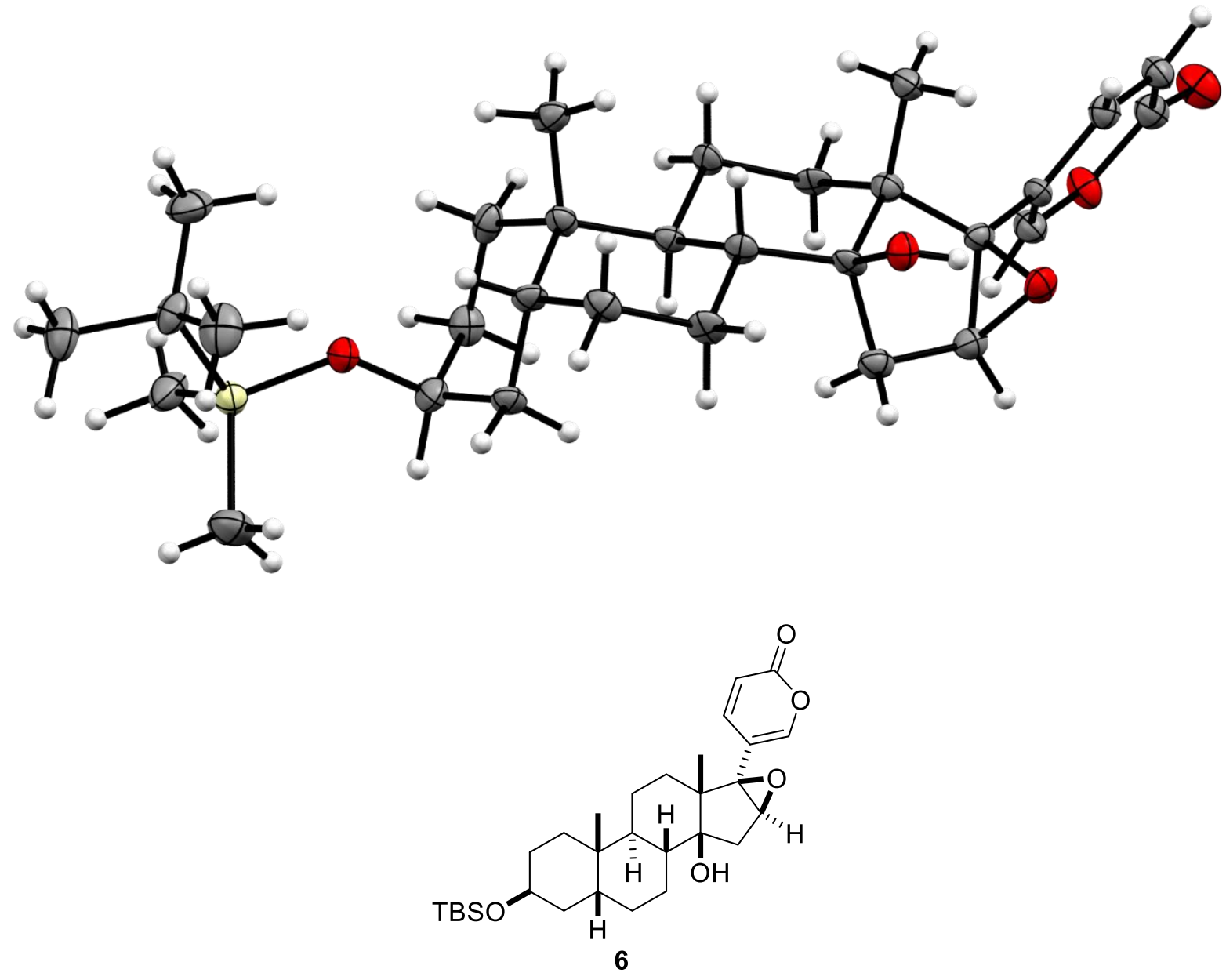

Figure S4. X-ray crystallographic structure of 6 (CCDC 2031223) with thermal ellipsoids at the $50 \%$ probability level (ORTEP) 

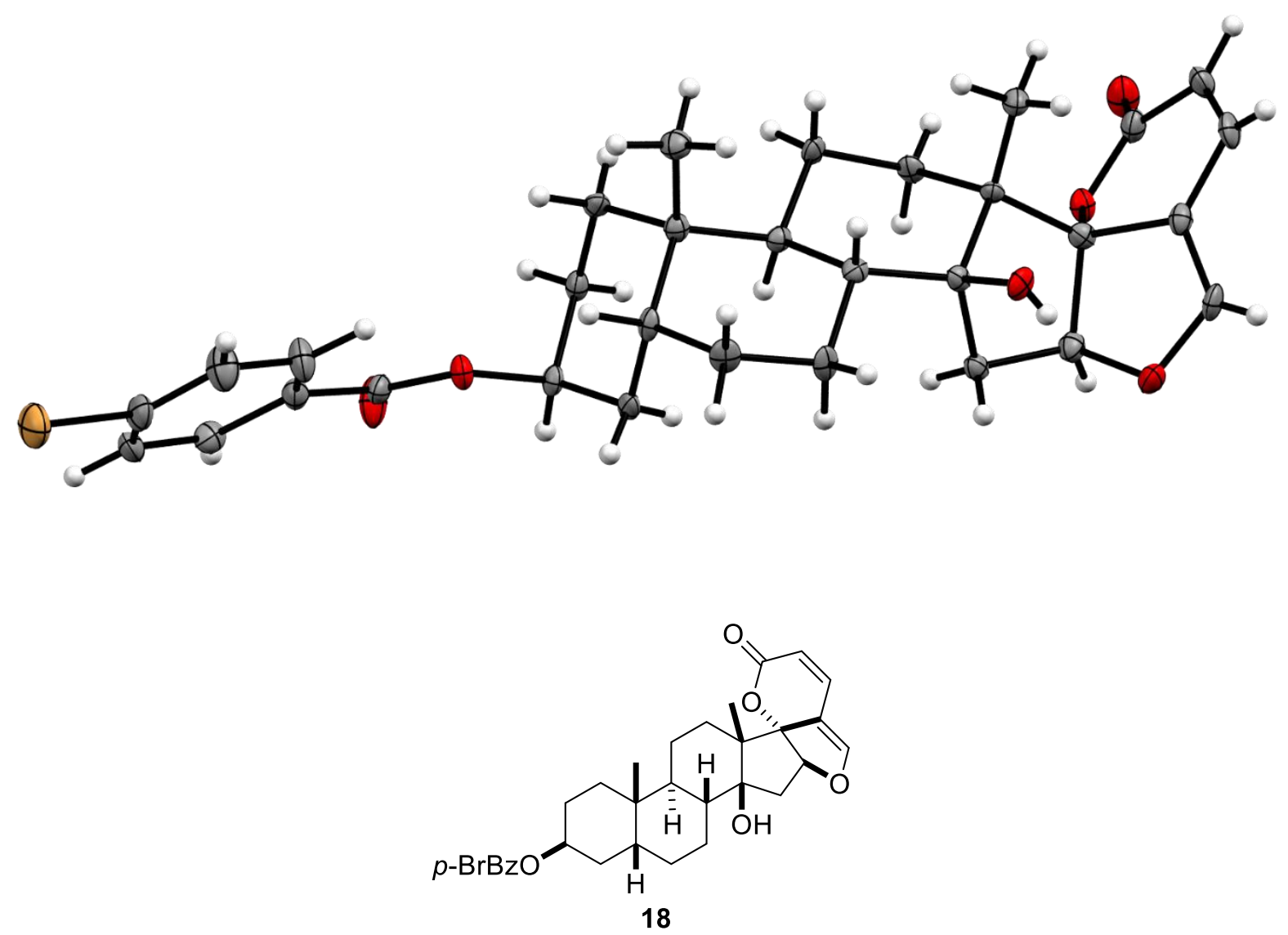

Figure S5. X-ray crystallographic structure of 18 (CCDC 2031224) with thermal ellipsoids at the $50 \%$ probability level (ORTEP) 
Table S10. Crystal data and structure refinement for $\mathbf{6}$ and $\mathbf{1 8}$

\begin{tabular}{|c|c|c|}
\hline Compound & 6 & 18 \\
\hline CCDC number & 2031223 & 2031224 \\
\hline Molecular formula & $\mathrm{C}_{30} \mathrm{H}_{46} \mathrm{O}_{5} \mathrm{Si}$ & $\mathrm{C}_{31} \mathrm{H}_{35} \mathrm{O}_{6} \mathrm{Br}$ \\
\hline Formula weight & 514.78 & 583.52 \\
\hline Temperature (K) & $93(2)$ & $296(1)$ \\
\hline Wavelength $(\AA)$ & 0.71075 & 0.71073 \\
\hline Crystal color, habit & colorless, prism & colorless, plate \\
\hline Crystal size $\left(\mathrm{mm}^{3}\right)$ & $0.230 \times 0.100 \times 0.050$ & $0.200 \times 0.200 \times 0.050$ \\
\hline Crystal system & orthorhombic & orthorhombic \\
\hline Space group & $\mathrm{P} 2{ }_{1}{ }_{1} 2_{1}(\# 19)$ & $\mathrm{P} 2{ }_{1}{ }_{1} 2_{1}(\# 19)$ \\
\hline Unit cell dimensions & & \\
\hline $\mathrm{a}(\AA)$ & $7.152(3)$ & $7.7359(3)$ \\
\hline $\mathrm{b}(\AA)$ & $12.733(5)$ & $12.9937(4)$ \\
\hline $\mathrm{c}(\AA)$ & $31.445(12)$ & $26.7557(14)$ \\
\hline$\alpha\left(^{\circ}\right)$ & 90.0000 & 90.0000 \\
\hline$\beta\left(^{\circ}\right)$ & 90.0000 & 90.0000 \\
\hline$\gamma\left(\left(^{\circ}\right)\right.$ & 90.0000 & 90.0000 \\
\hline Volume $\left(\AA^{3}\right)$ & $2864(2)$ & $2689.43(19)$ \\
\hline$Z$ & 4 & 4 \\
\hline Density (calculated) $\left(\mathrm{g} / \mathrm{cm}^{3}\right)$ & 1.194 & 1.441 \\
\hline$\mu(\mathrm{MoK} \alpha)\left(\mathrm{cm}^{-1}\right)$ & 1.180 & 15.769 \\
\hline $\mathrm{F}(000)$ & 1120.00 & 1216.00 \\
\hline Index ranges & $-9<=\mathrm{h}<9$ & $-8<=\mathrm{h}<10$ \\
\hline & $-16<=\mathrm{k}<13$ & $-17<=\mathrm{k}<16$ \\
\hline & $-29<=1<40$ & $-31<=1<35$ \\
\hline Reflections collected & 22448 & 18355 \\
\hline Independent reflections & 6545 & 6093 \\
\hline $\mathrm{R}$ (int) & 0.1066 & 0.0354 \\
\hline Completeness to theta & $27.483^{\circ}, 100 \%$ & $29.094^{\circ}, 100 \%$ \\
\hline Max. and min. transmission & $0.994,0.773$ & $1.000,0.841$ \\
\hline Refinement method & \multicolumn{2}{|c|}{ Full-matrix least-squares on $\mathrm{F}^{2}$} \\
\hline No. Observations (All reflections) & 6545 & 6093 \\
\hline No. Variables & 463 & 446 \\
\hline Reflection/Parameter Ratio & 14.14 & 13.66 \\
\hline Goodness-of-fit on $\mathrm{F}^{2}$ & 1.063 & 1.025 \\
\hline Residuals: R1 $(I>2.00 \sigma(I))$ & 0.0758 & 0.0416 \\
\hline Residuals: R (All reflections) & 0.1104 & 0.0544 \\
\hline Residuals: wR2 (All reflections) & 0.1470 & 0.1031 \\
\hline $\begin{array}{l}\text { Max. and min. peak in Final Diff. } \\
\text { Map }\left(\mathrm{e}-/ \AA^{3}\right)\end{array}$ & $0.25,-0.30$ & $0.89,-0.49$ \\
\hline
\end{tabular}




\section{Biological experiments}

Cell culture. MCF-7 human breast cancer cells were obtained from the American Type Culture Collection (ATCC). The cells were maintained with growth medium [Dulbecco's Modified Eagle's Medium (DMEM) low glucose (D6046, Sigma) supplemented with 10 v/v\% heat-inactivated fetal bovine serum, penicillin $\mathrm{G}$ (100 units $/ \mathrm{mL})$, and streptomycin (100 $\mu \mathrm{g} / \mathrm{mL})]$ under atmosphere of $5 \% \mathrm{CO}_{2}$ at $37{ }^{\circ} \mathrm{C}$. The growth medium was refreshed every 2 or 3 days to reach $70-90 \%$ cell confluence.

Sulforhodamine B assay. Growth inhibitory activities of the tested compounds were evaluated according to the literature. ${ }^{\mathrm{S} 14}$ Cell growth $(\%)$ was defined as follows:

$$
\begin{array}{ll}
\text { cell growth }(\%)=\frac{F L(\text { sample })-F L(\text { day } 0)}{F L(\text { control })-F L(\text { day } 0)} \times 100 & \{F L(\text { sample }) \geq F L(\text { day } 0)\} \\
\text { cell growth }(\%)=\frac{F L(\text { sample })-F L(\text { day } 0)}{F L(\text { day } 0)} \times 100 & \{F L(\text { day } 0)>F L(\text { sample })\}
\end{array}
$$

$F L=$ mean of the fluorescence intensity (Ex. $485 \mathrm{~nm} / \mathrm{Em} .585 \mathrm{~nm})$

day $0=$ time of addition of the tested compound as serial dilutions

control $=$ control wells treated by vehicle $(\mathrm{DMSO})$

Various concentrations of compounds in the growth medium containing 2\% DMSO were prepared by serial dilutions. MCF-7 cells were cultured in $6 \mathrm{~cm}$ cell culture dishes filled with the growth medium and harvested by trypsinization at $37^{\circ} \mathrm{C}$ for $5-10 \mathrm{~min}$. The collected cells were resuspended into the growth medium at $1.25 \times 10^{5}$ cells $/ \mathrm{mL}$. The cell suspension $(100$ $\mu \mathrm{L} /$ well) was seeded into the black polystyrene flat-bottom 96-well plates (sample-plates, 655090, Greiner bio-one). For calculating $F L($ day0), the same cell suspension (100 $\mu \mathrm{L} /$ well) was seeded into an independent 96-well plate (day0-plate). The sample-plate and day0-plate were incubated under atmosphere of $5 \% \mathrm{CO}_{2}$ at $37^{\circ} \mathrm{C}$ for $24 \mathrm{~h}$. Aliquots of the former medium $(100 \mu \mathrm{L})$ containing compounds were added to each well of the sample-plate. The growth medium containing $2 \%$ DMSO $(100 \mu \mathrm{L})$ was added to the wells for calculating $F L$ (control) of the sample-plate and the wells for calculating $F L($ day 0$)$ of the day0-plate. To the cells in day0-plate was added an ice-cold solution of $30 \mathrm{w} / \mathrm{v} \%$ trichloroacetic acid (TCA) in $\mathrm{H}_{2} \mathrm{O}$ (100 $\mu \mathrm{L} /$ well). The day0-plate was incubated at $4{ }^{\circ} \mathrm{C}$ for 60 min, washed with $\mathrm{H}_{2} \mathrm{O}$ (x4), dried, and stored at room temperature. The sample-plate was incubated at $37{ }^{\circ} \mathrm{C}$ under atmosphere of $5 \% \mathrm{CO}_{2}$ for $48 \mathrm{~h}$. To the cells in the sample-plate was added an ice-cold solution of $30 \mathrm{w} / \mathrm{v} \%$ TCA in $\mathrm{H}_{2} \mathrm{O}\left(100 \mu \mathrm{L} /\right.$ well). The sample-plate was incubated at $4{ }^{\circ} \mathrm{C}$ for $60 \mathrm{~min}$, washed with $\mathrm{H}_{2} \mathrm{O}$ ( $\mathrm{x} 4$ ), and dried. To each well of the day0-plate and sample-plate was added a solution of sulforhodamine $\mathrm{B}$ in $\mathrm{H}_{2} \mathrm{O}(570 \mu \mathrm{g} / \mathrm{mL}, 100 \mu \mathrm{L} /$ well $)$. The fixed cells were stained at room temperature for $30 \mathrm{~min}$ in the dark. The cells were washed with $\mathrm{AcOH} / \mathrm{H}_{2} \mathrm{O}(1 / 99, \mathrm{x} 4)$ and dried. To the stained cells was added a solution of 2-amino-2-(hydroxymethyl)-1,3propanediol (Tris) in $\mathrm{H}_{2} \mathrm{O}(10 \mathrm{mM}, 200 \mu \mathrm{L} /$ well $)$. The plates were vortexed at room temperature for $10 \mathrm{~min}$. The fluorescence (Ex. $485 \mathrm{~nm} / \mathrm{Em}$. $585 \mathrm{~nm}$ ) of each well was measured on Spectra Max Gemini EM microplate reader (Molecular Devices). Growth inhibitory activities of the tested compounds were evaluated as $50 \%$ growth inhibitory 
concentration (GI50) values $(\mathrm{nM})$ by means of three replicates. Sigmoidal curve fittings were performed on GraphPad Prism (GraphPad Software, Figure S6). The mean GI50 values and standard deviations of ouabain, 1-5, and $\mathbf{2 8}$ were determined from three independent experiments (Table S11).

Table S11. Growth inhibitory activity of ouabain and bufadienolides

\begin{tabular}{cc} 
compound & $\mathrm{GI}_{50}[\mathrm{nM}]^{a}$ \\
\hline ouabain $\left(\right.$ control) $^{a}$ & $20.5 \pm 1.1$ \\
ouabain $^{\text {S15 }}$ & $19.9 \pm 3.6$ \\
$\mathbf{1}$ & $13.3 \pm 1.8$ \\
$\mathbf{2}$ & $55.0 \pm 5.7$ \\
$\mathbf{3}$ & $28.2 \pm 3.9$ \\
$\mathbf{4}$ & $301 \pm 36$ \\
$\mathbf{5}$ & $1190 \pm 340$ \\
$\mathbf{2 8}$ & $2390 \pm 670$
\end{tabular}

${ }^{a}$ Three independent experiments were performed (mean \pm SD). 

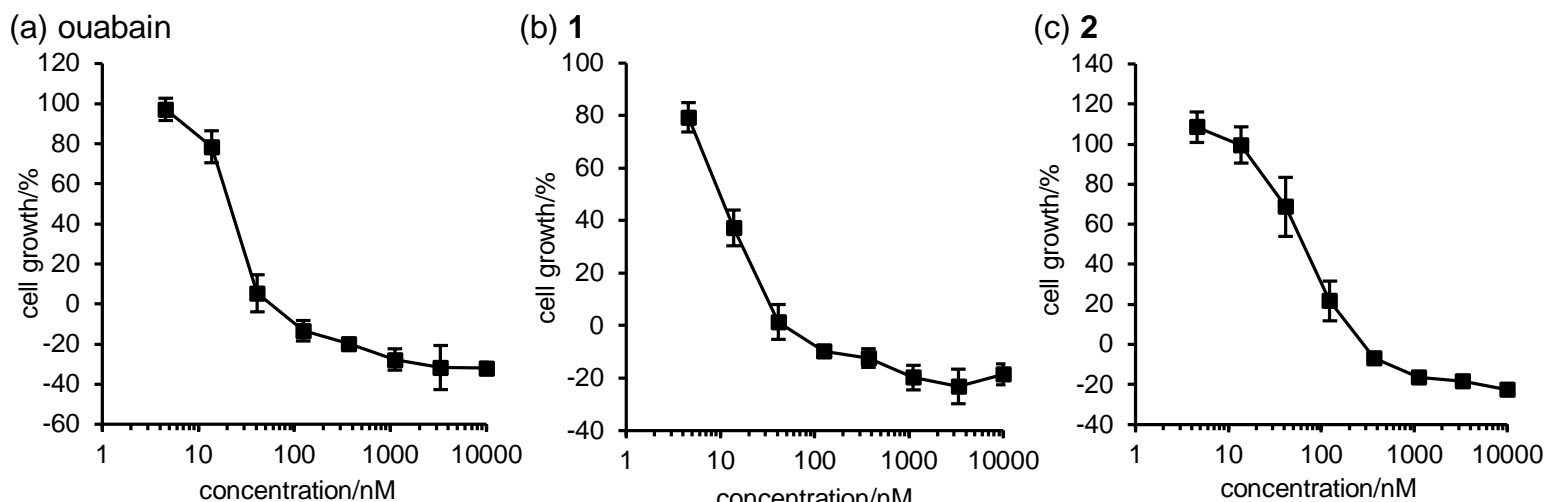

(d) 3

(e) 4

(f) 5
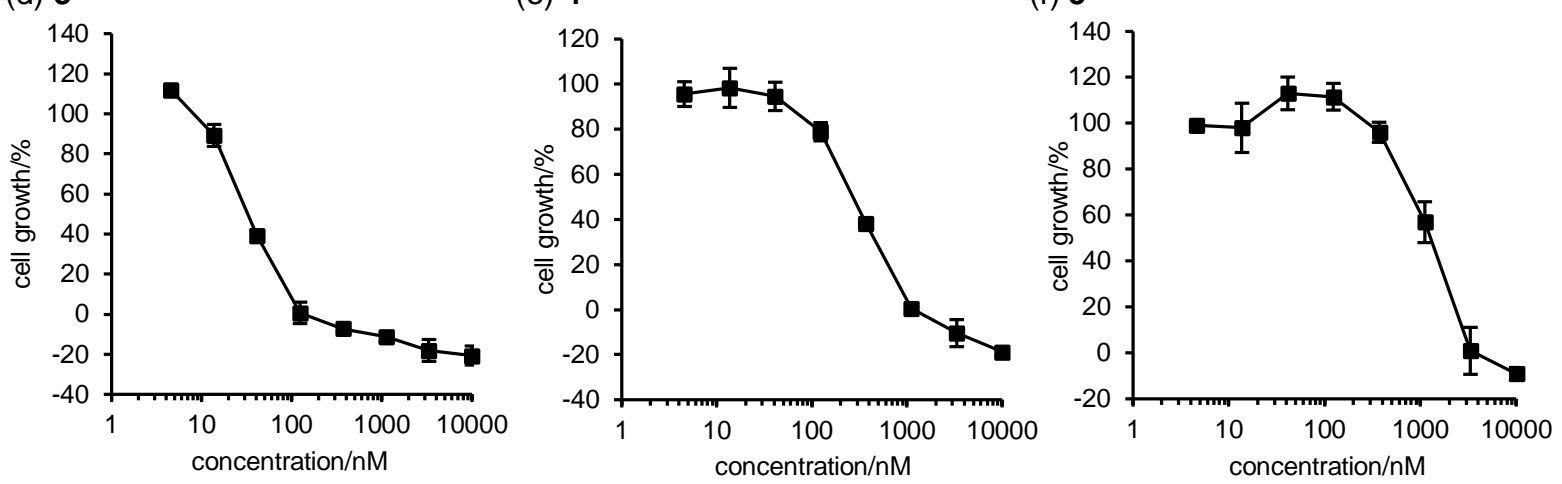

(g) 28

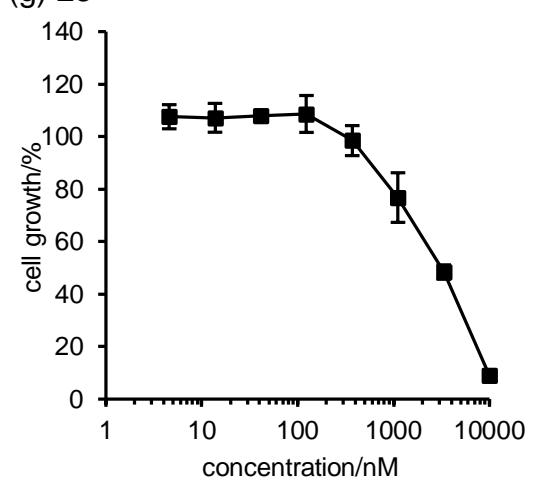

Figure S6. Representative concentration-response curves of ouabain, 1-5, and 28. Ouabain was used as a positive control. The cells were incubated for $48 \mathrm{~h}$ in the presence of compounds, and the cell growth (\%) was evaluated. Each plot is displayed as mean \pm SD of three replicates. 


\section{References}

S1. Tsuji, N.; Suzuki, J.; Shiota, M. Highly Stereoselective Hydrogenation of 3-Oxo-4-ene and -1,4-diene Steroids to $5 \beta$ Compounds with Palladium Catalyst. J. Org. Chem. 1980, 45, 2729-2731.

S 2. Wong, M.-K.; Chung, N.-W.; He, L.; Wang, X.-C.; Yan, Z.; Tang, Y.-C.; Yang, D. Investigation on the Regioselectivities of Intramolecular Oxidation of Unactivated $\mathrm{C}-\mathrm{H}$ Bonds by Dioxiranes Generated in Situ. J. Org. Chem. 2003, 68, 6321-6328.

S3. Göndös, G.; Orr, J. C. Stereoselective and Regioselective Reduction of Steroid Ketones by Potassium Tri(R,S-s-butyl)borohydride. J. Chem. Soc., Chem. Commun. 1982, 1239-1240.

S4. Hilton, P. J.; McKinnon, W.; Gravett, E. C.; Peron, J.-M. R.; Frampton, C. M.; Nicholls, M. G.; Lord, G. Selective Inhibition of the Cellular Sodium Pump by Emicymarin and $14 \beta$ Anhydroxy Bufadienolides. Steroids 2010, 75, 1137-1145.

S5. Afarinkia, K.; Posner, G. H. 5-Bromo-2-pyrone: An Easily Prepared Ambiphilic Diene and a Synthetic Equivalent of 2-Pyrone in Mild, Thermal, Diels-Alder Cycloadditions. Tetrahedron Lett. 1992, 33, 7839-7842.

S6. Pettit, G. R.; Moser, B. R.; Mendonça, R. F.; Knight, J. C.; Hogan, F. The Cephalostatins. 22. Synthesis of Bis-steroidal Pyrazine Pyrones. J. Nat. Prod. 2012, 75, 1063-1069.

S7. Wang, W.; Yamnitz, C. R.; Gokel, G. W. Heterocyclic Amide Hydraphile Synthetic Cation Transporters. Heterocycles 2007, 73, 825-839.

S8. Pettit, G. R.; Kamano, Y.; Drašar, P.; Inoue, M.; Knight, J. C. Synthesis of Bufalitoxin and Bufotoxin. J. Org. Chem. 1987, 52, 3573-3578.

S9. Oger, F.; Lecorgne, A.; Sala, E.; Nardese, V.; Demay, F.; Chevance, S.; Desravines, D. C.; Aleksandrova, N.; Le Guével, R.; Lorenzi, S.; Beccari, A. R.; Barath, P.; Hart, D. J.; Bondon, A.; Carettoni, D.; Simonneaux, G.; Salbert, G. Biological and Biophysical Properties of the Histone Deacetylase Inhibitor Suberoylanilide Hydroxamic Acid Are Affected by the Presence of Short Alkyl Groups on the Phenyl Ring. J. Med. Chem. 2010, 53, 1937-1950.

S10. Wang, Y.-M.; Li, Z.-Y.; Wang, J.-J.; Wu, X.-Y.; Gao, H.-M.; Wang, Z.-M. Bufadienolides and Polyhydroxycholestane Derivatives from Bufo Bufo Gargarizans. J. Asian Nat. Prod. Res. 2015, 17, 364-376.

S11. Córdova, W. H. P.; Leitão, S. G.; Cunha-Filho, G.; Bosch, R. A.; Alonso, I. P.; PeredaMiranda, R.; Gervou, R.; Touza, N. A.; Quintas, L. E. M.; Noël, F. Bufadienolides from Parotoid Gland Secretions of Cuban Toad Peltophryne Fustiger (Bufonidae): Inhibition of Human Kidney $\mathrm{Na}^{+} / \mathrm{K}^{+}$-ATPase Activity. Toxicon 2016, 110, 27-34.

S12. Liu, J.; Zhang, D.; Li, Y.; Chen, W.; Ruan, Z.; Deng, L.; Wang, L.; Tian, H.; Yiu, A.; Fan, C.; Luo, H.; Liu, S.; Wang, Y.; Xiao, G.; Chen, L.; Ye, W. Discovery of Bufadienolides as a Novel Class of ClC-3 Chloride Channel Activators with Antitumor Activities. J. Med. Chem. 2013, 56, 5734-5743.

S13. Banuls, L. M. Y.; Urban, E.; Gelbcke, M.; Dufrasne, F.; Kopp, B.; Kiss, R.; Zehl, M. Structure-Activity Relationship Analysis of Bufadienolide-Induced in Vitro Growth Inhibitory Effects on Mouse and Human Cancer Cells. J. Nat. Prod. 2013, 76, 1078-1084.

S14. Vichai, V.; Kirtikara, K. Sulforhodamine B Colorimetric Assay for Cytotoxicity Screening. Nat. Protoc. 2006, 1, 1112-1116.

S15. Urabe, D.; Nakagawa, Y.; Mukai, K.; Fukushima, K.; Aoki, N.; Itoh, H.; Nagatomo, M.; Inoue, M. Total Synthesis and Biological Evaluation of 19-Hydroxysarmentogenin-3-O- $\alpha$-Lrhamnoside, Trewianin, and Their Aglycons. J. Org. Chem. 2018, 83, 13888-13910. 


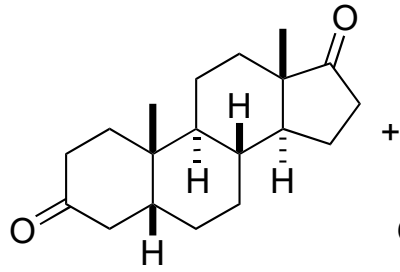

$10 \mathrm{a}$<smiles>CC12CCC3C(CCC4CC(=O)CCC43C)C1CCC2=O</smiles>

$10 \mathrm{~b}$

$10 \mathrm{a}: 10 \mathrm{~b}=9.3: 1$

${ }^{1} \mathrm{H}$ NMR $\left(500 \mathrm{MHz}, \mathrm{CD}_{3} \mathrm{OD}\right)$

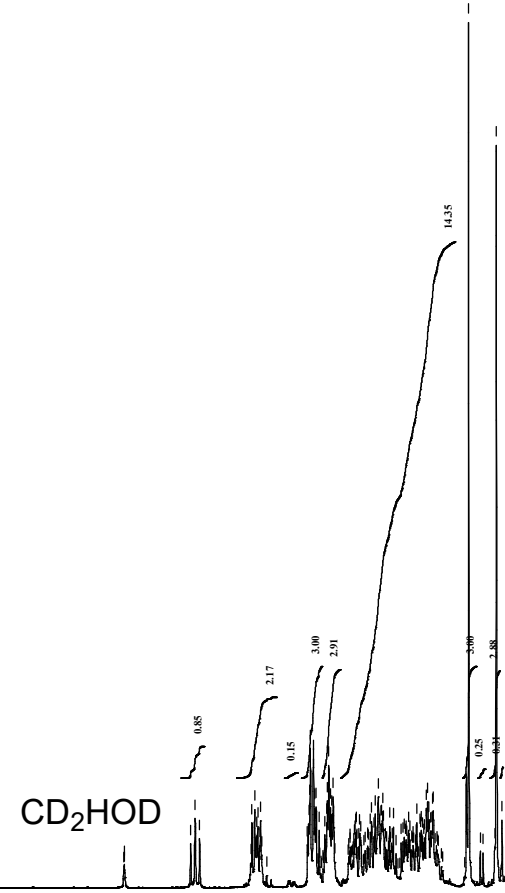

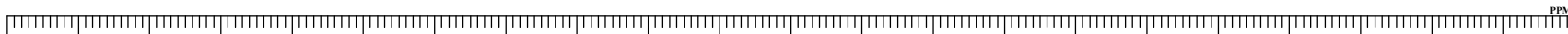<smiles>CC12CCC3C(CCC4CC(=O)CCC43C)C1CCC2=O</smiles>

$10 \mathrm{a}$<smiles>CC12CCC3[C@H](CC[C@@H]4CC(=O)CCC34C)[C@@H]1CCC2=O</smiles>

$10 \mathrm{~b}$

$10 \mathrm{a}: 10 \mathrm{~b}=9.3: 1$

${ }^{13} \mathrm{C}\left\{{ }^{1} \mathrm{H}\right\} \mathrm{NMR}\left(125 \mathrm{MHz}, \mathrm{CD}_{3} \mathrm{OD}\right)$
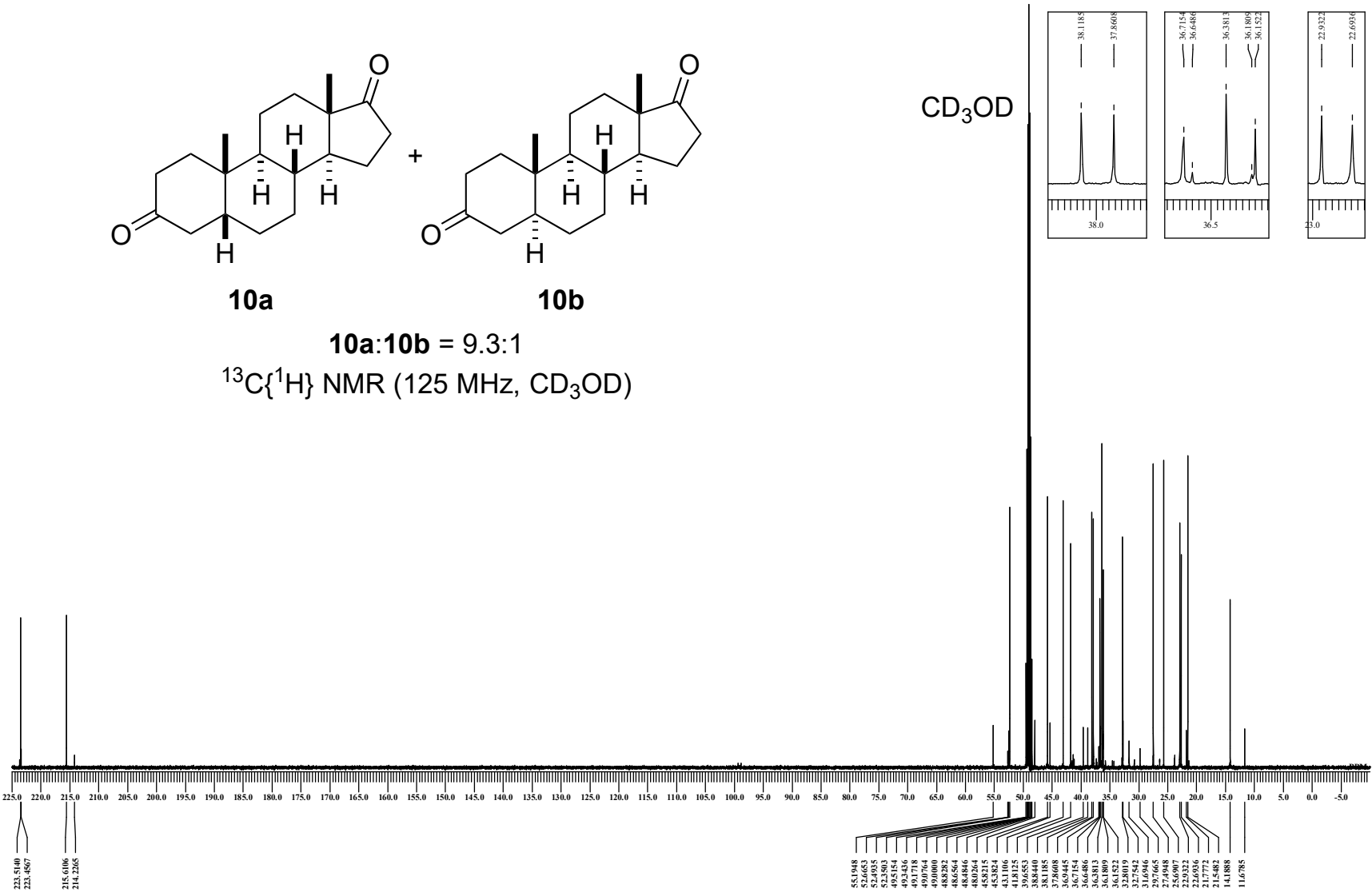
<smiles>CC12CCC3C(CCC4(C)C3CCC3(C)C5CCC(O)CC5CCC34)C1CCC2=O</smiles>

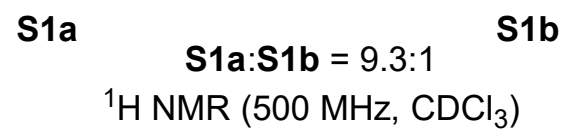

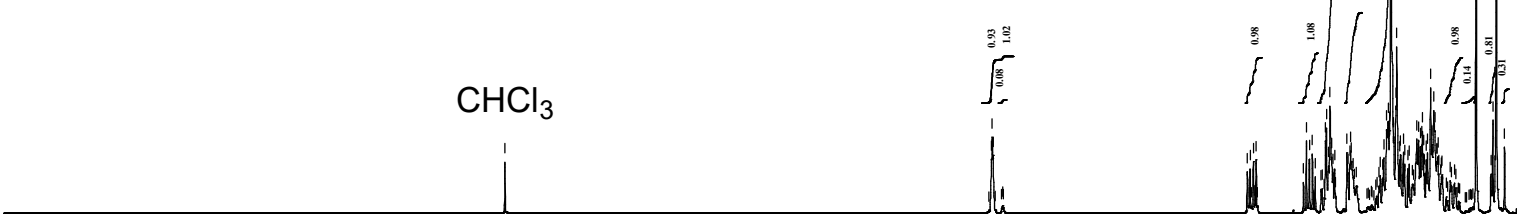

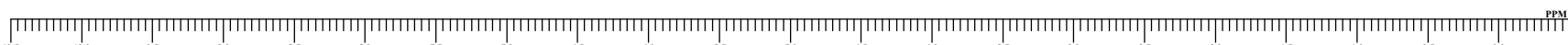

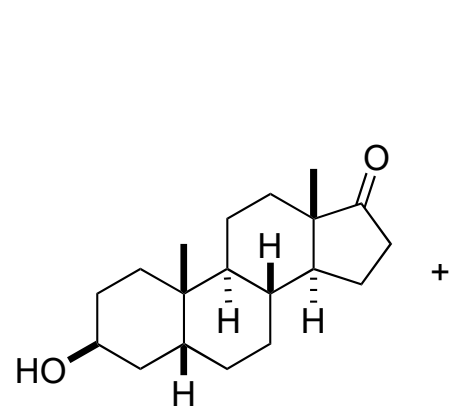

S1a<smiles>CC12CCC3C(=O)CC[C@@H]3C1CC[C@H]1CC(O)CC[C@@H]12</smiles>

S1b

S1a:S1b $=9.3: 1$

${ }^{13} \mathrm{C}\left\{{ }^{1} \mathrm{H}\right\}$ NMR $\left(125 \mathrm{MHz}, \mathrm{CDCl}_{3}\right)$

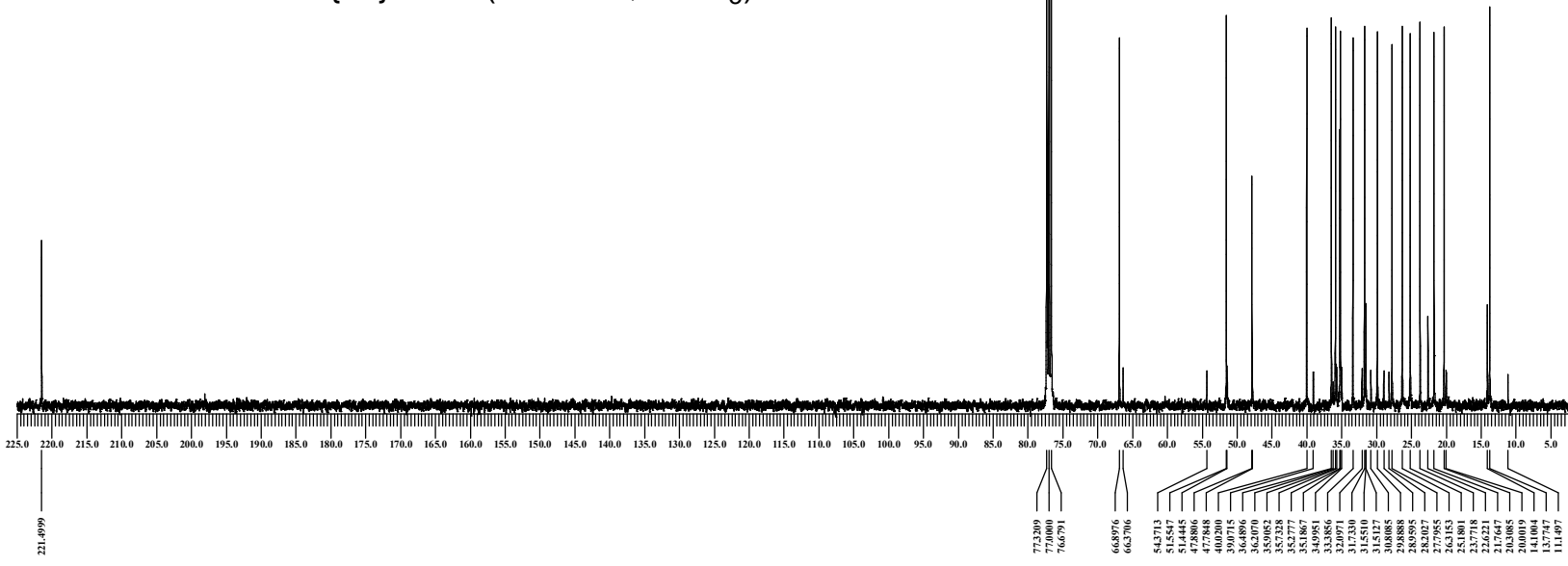




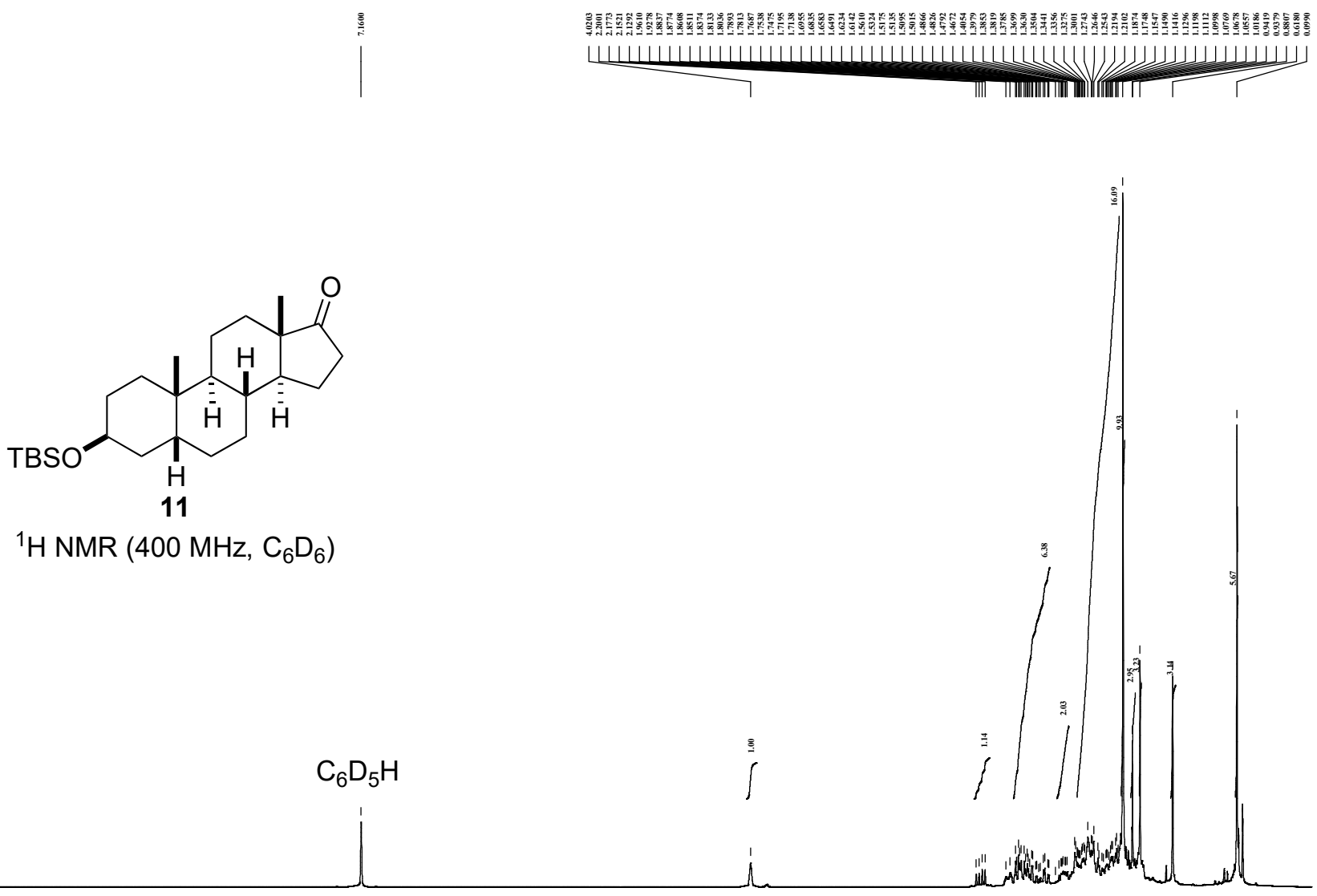

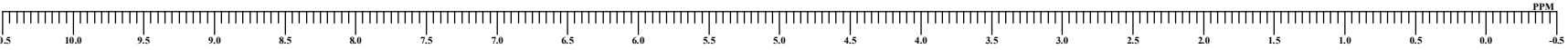

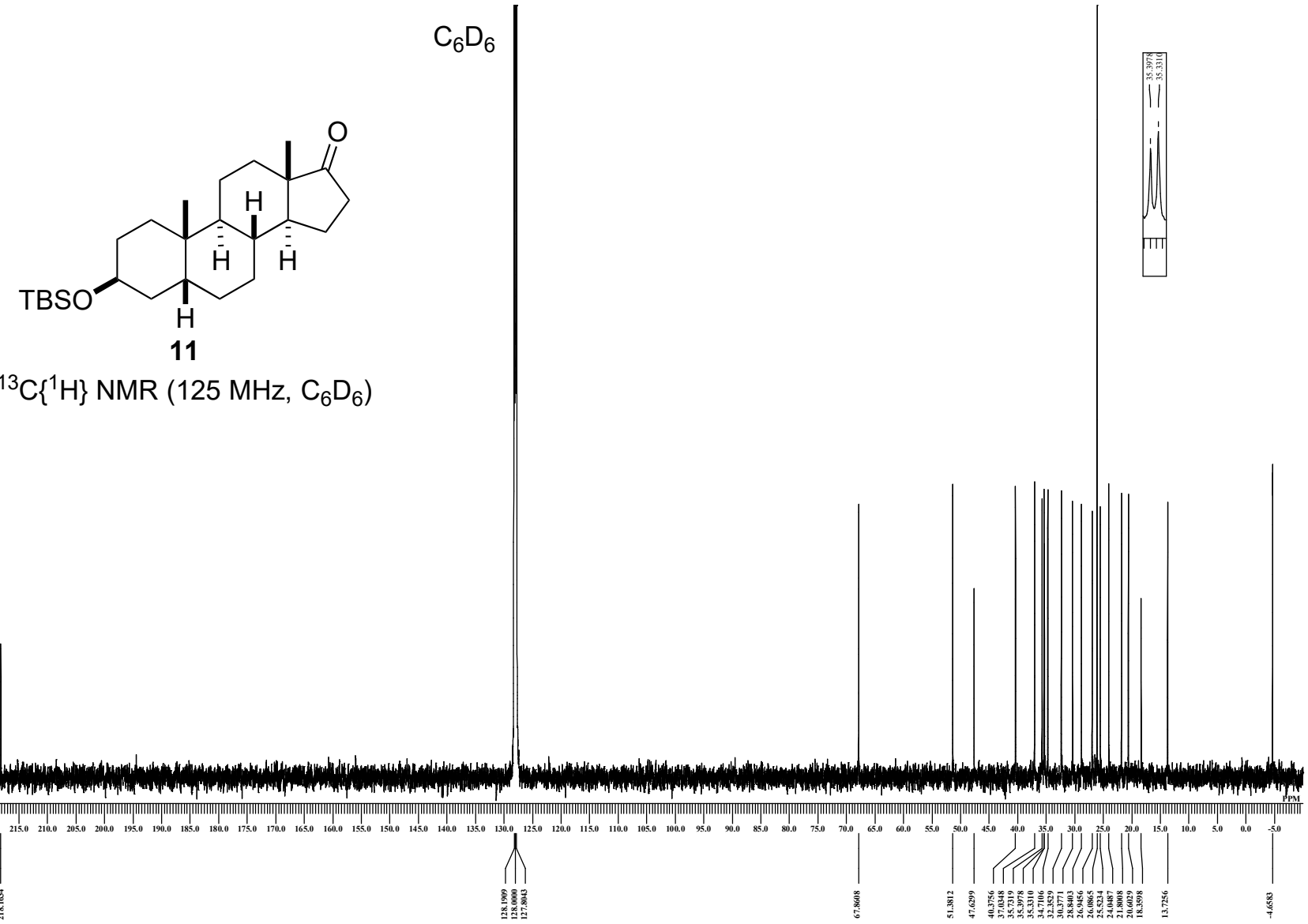




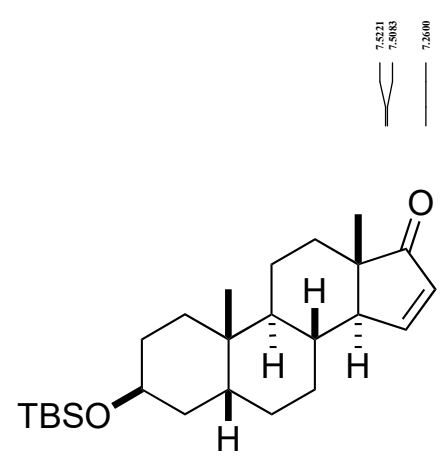

12

${ }^{1} \mathrm{H}$ NMR $\left(400 \mathrm{MHz}, \mathrm{CDCl}_{3}\right)$

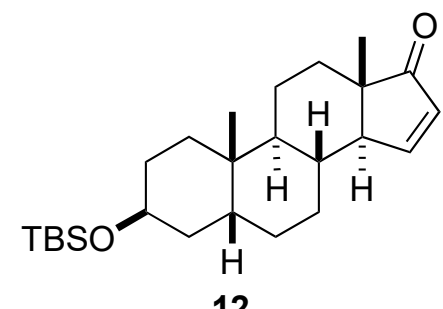

${ }^{13} \mathrm{C}\left\{{ }^{1} \mathrm{H}\right\} \operatorname{NMR}\left(100 \mathrm{MHz}, \mathrm{CDCl}_{3}\right)$ 

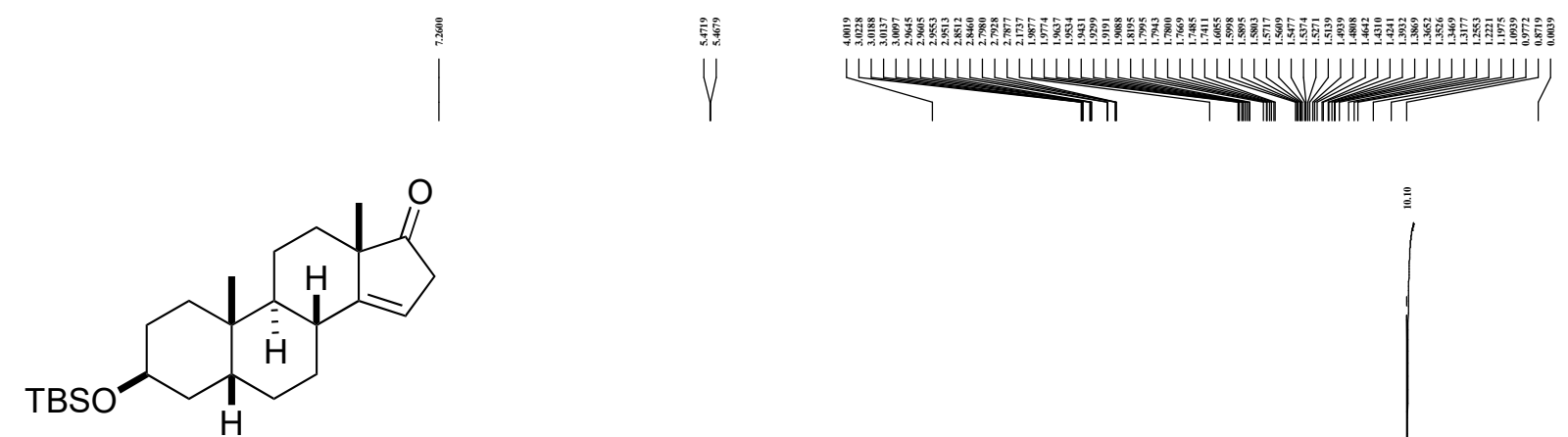

13

${ }^{1} \mathrm{H}$ NMR $\left(400 \mathrm{MHz}, \mathrm{CDCl}_{3}\right)$

$\mathrm{CHCl}_{3}$

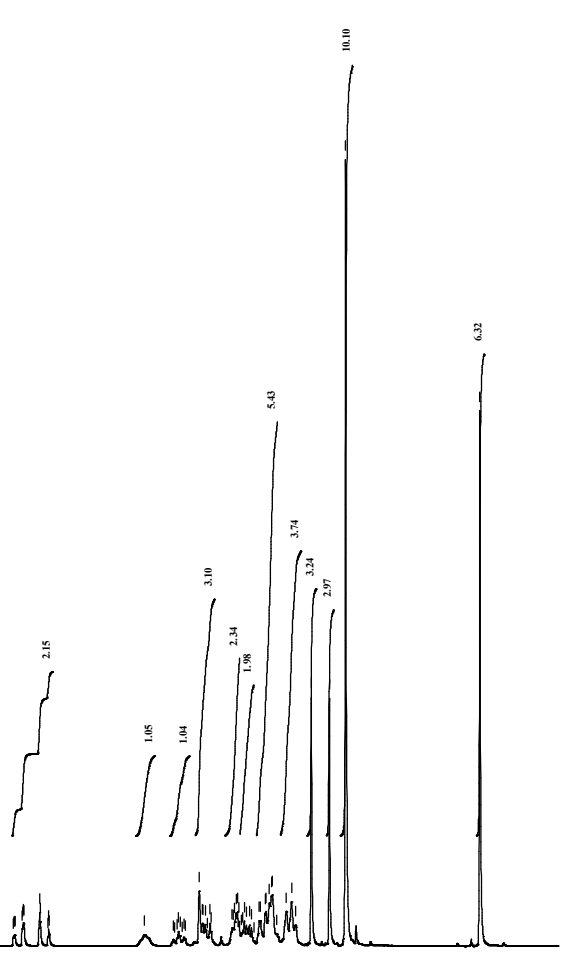

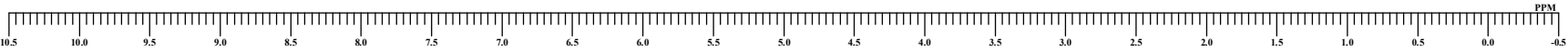

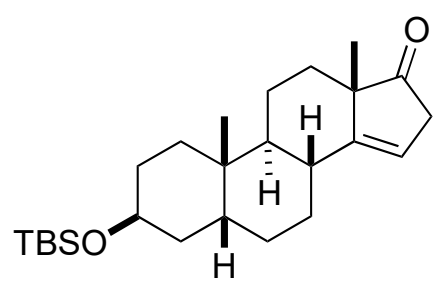

13

${ }^{13} \mathrm{C}\left\{{ }^{1} \mathrm{H}\right\} \operatorname{NMR}\left(100 \mathrm{MHz}, \mathrm{CDCl}_{3}\right)$

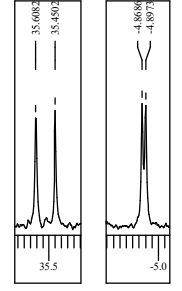

$\mathrm{CDCl}_{3}$
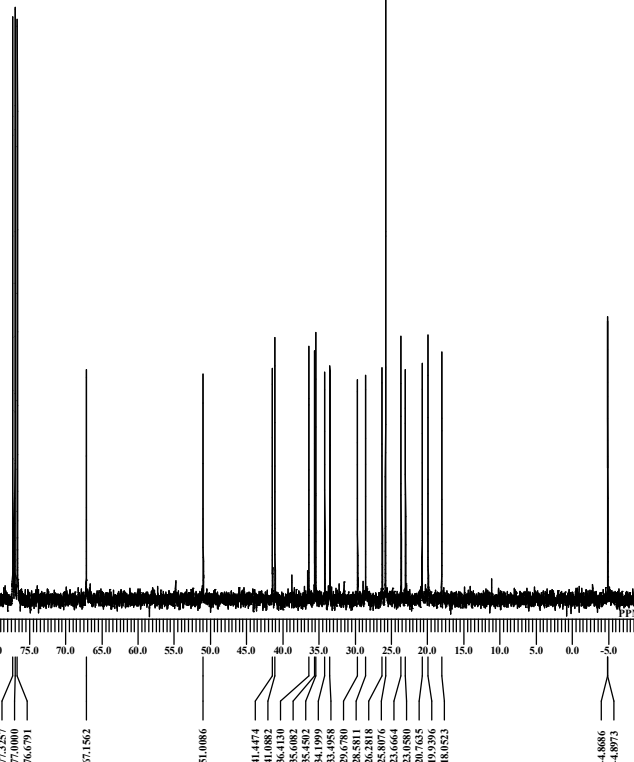


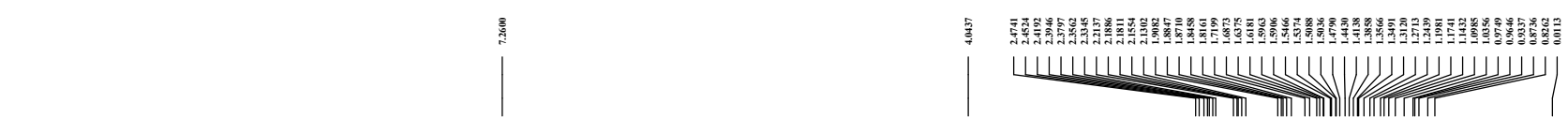

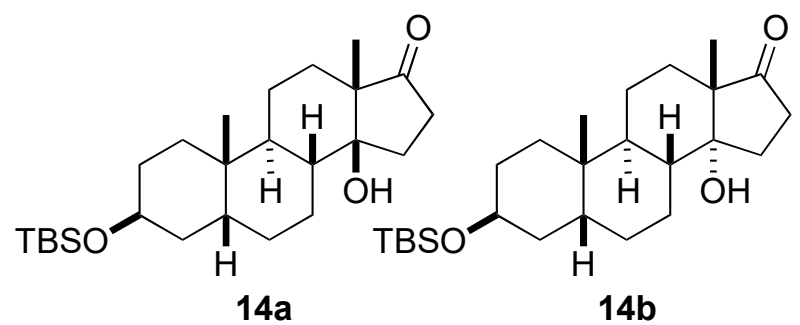

$14 a: 14 b=2.3: 1$

${ }^{1} \mathrm{H}$ NMR $\left(400 \mathrm{MHz}, \mathrm{CDCl}_{3}\right)$

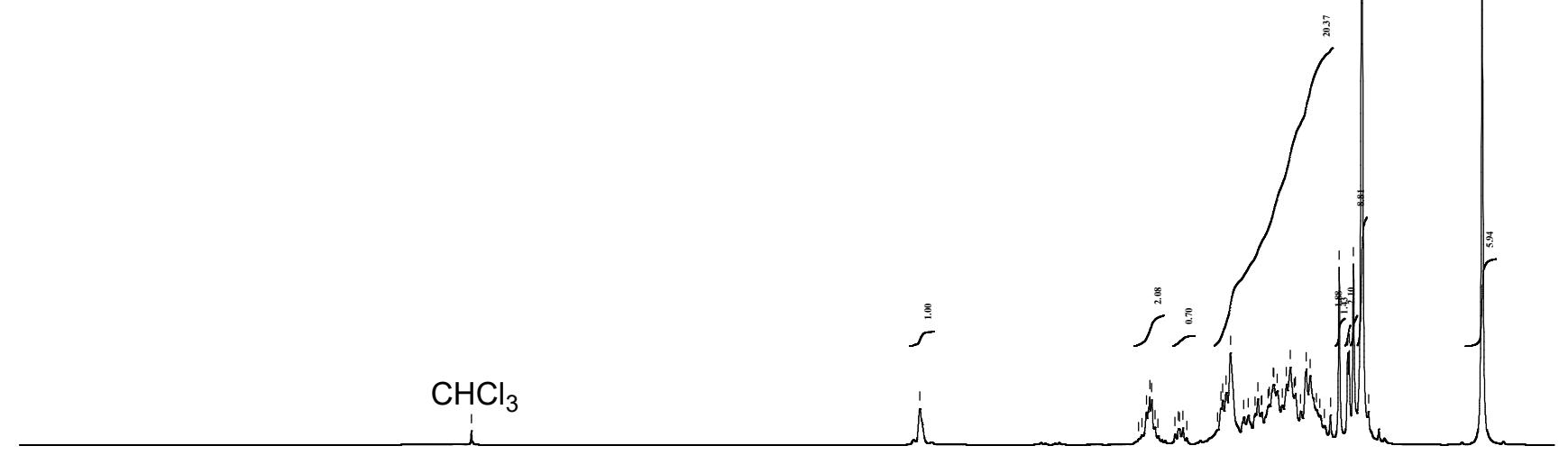

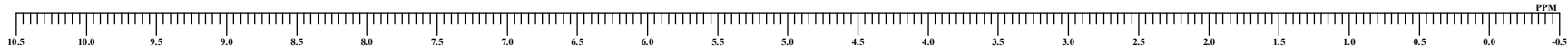

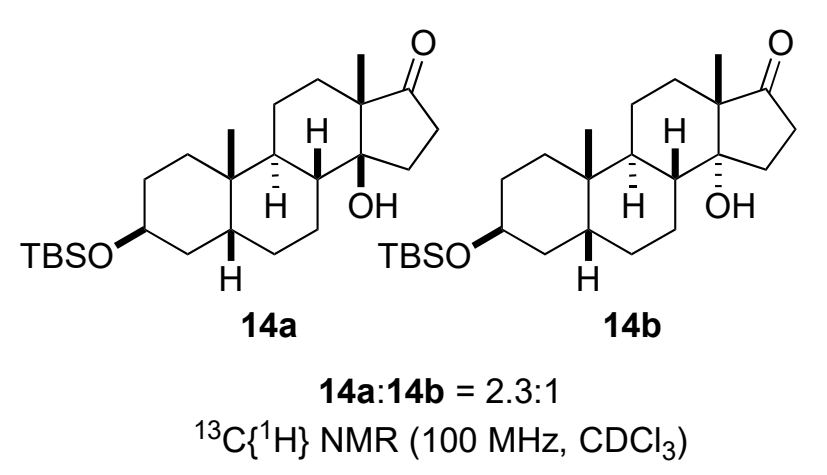
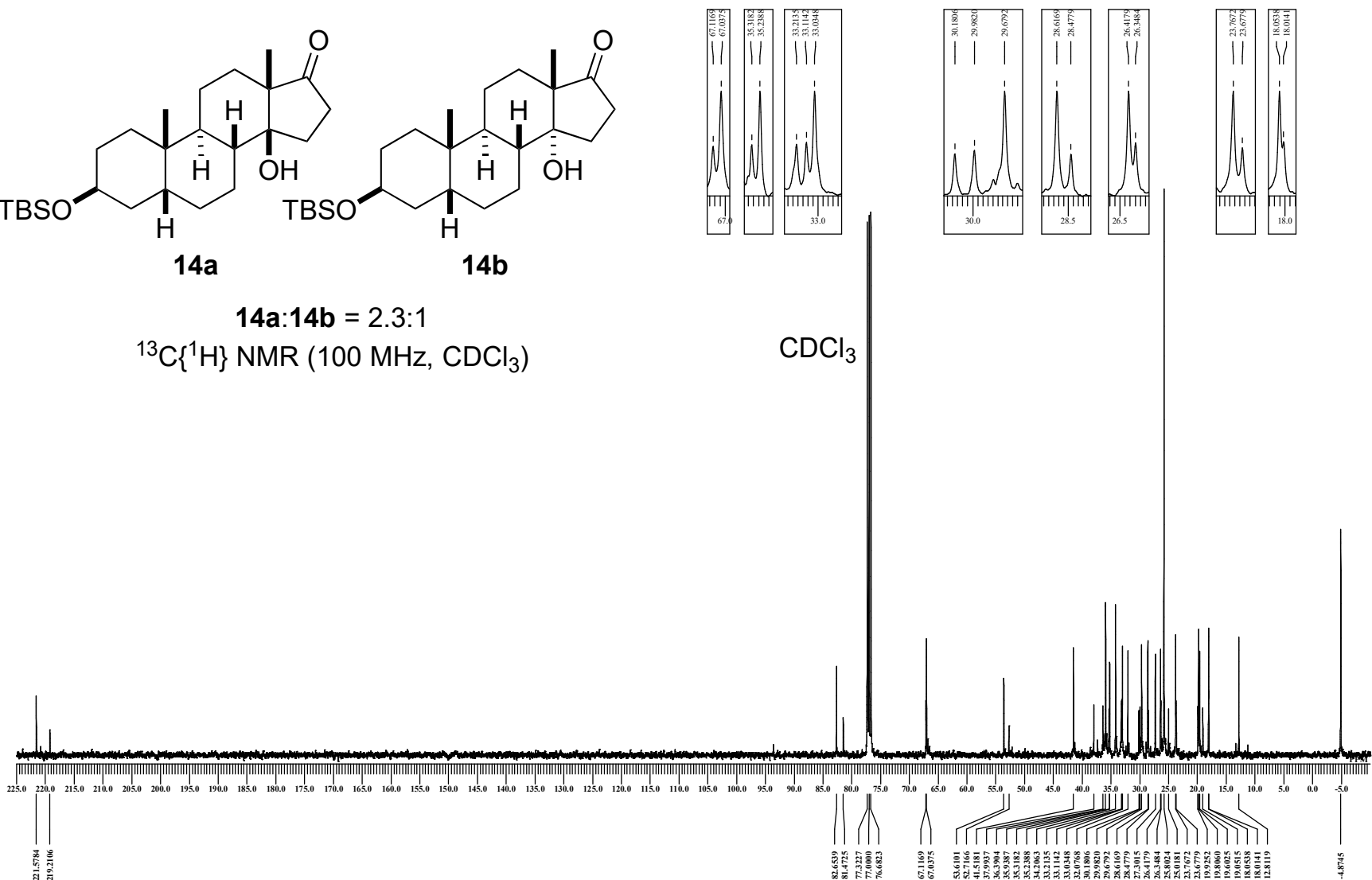

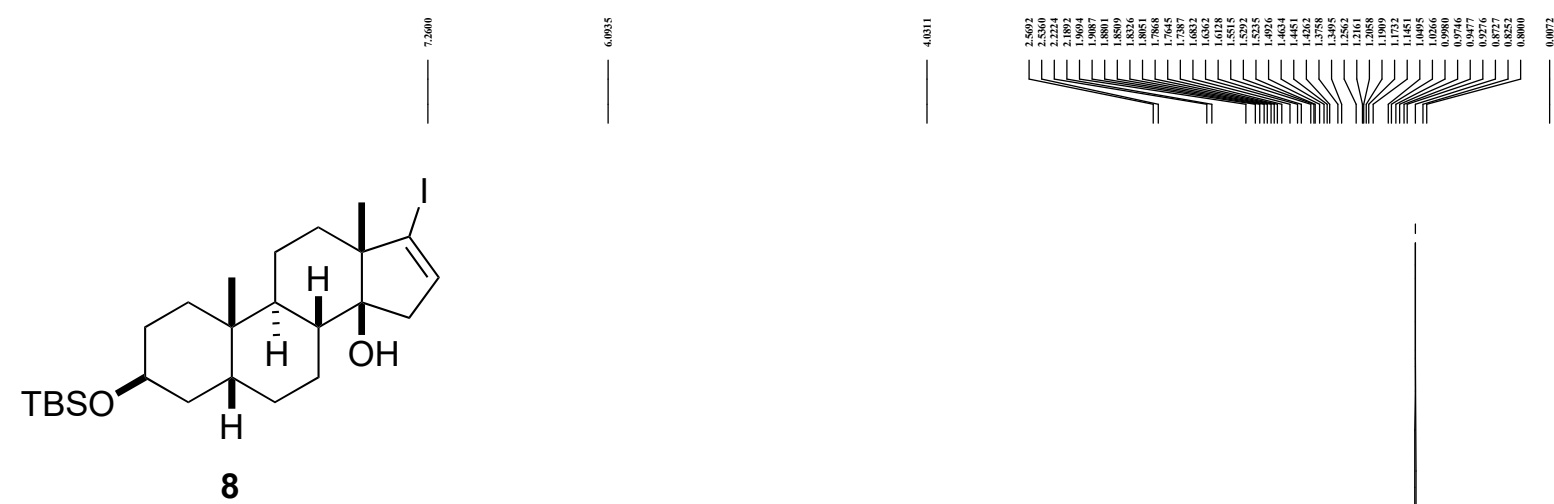

H NMR $\left(500 \mathrm{MHz}, \mathrm{CDCl}_{3}\right)$
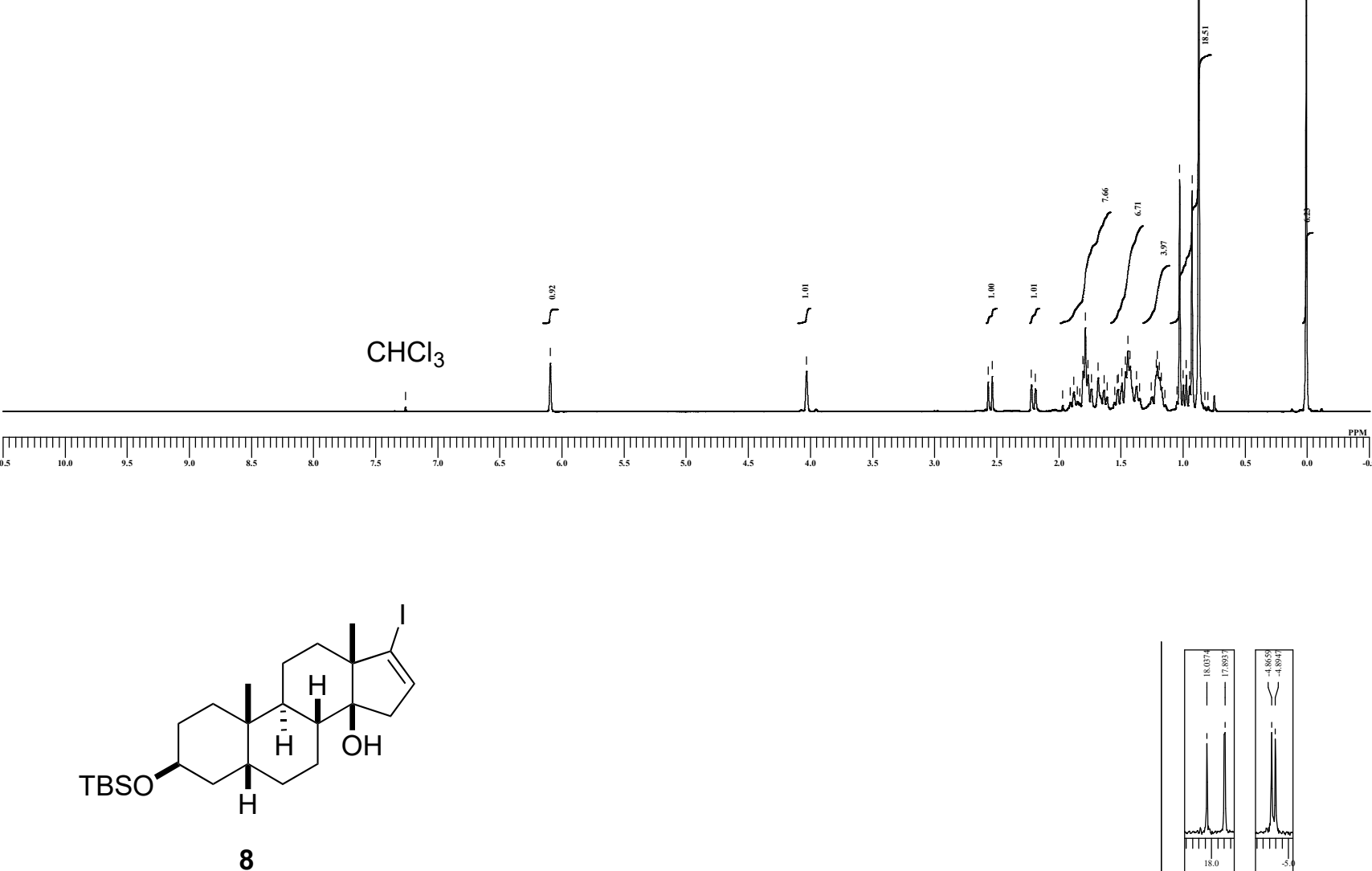

${ }^{13} \mathrm{C}\left\{{ }^{1} \mathrm{H}\right\} \mathrm{NMR}\left(125 \mathrm{MHz}, \mathrm{CDCl}_{3}\right)$

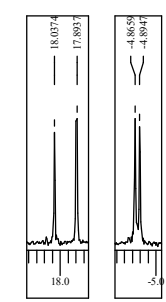




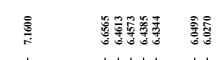 \\ iU}<smiles>C[AsH2]c1ccc(=O)oc1</smiles>

${ }^{1} \mathrm{H}$ NMR $\left(400 \mathrm{MHz}, \mathrm{C}_{6} \mathrm{D}_{6}\right)$

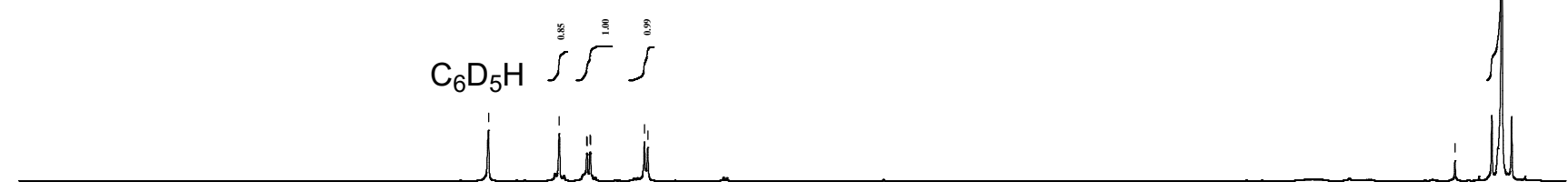<smiles>C[AsH2]c1ccc(=O)oc1</smiles>

$\mathrm{C}_{6} \mathrm{D}_{6}$

${ }^{13} \mathrm{C}\left\{{ }^{1} \mathrm{H}\right\}$ NMR $\left(100 \mathrm{MHz}, \mathrm{C}_{6} \mathrm{D}_{6}\right)$

(1)

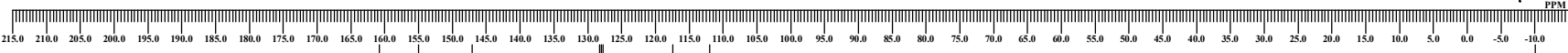

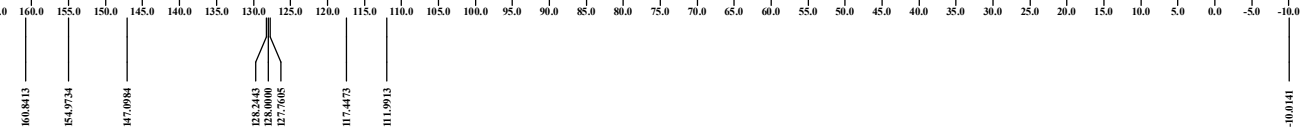



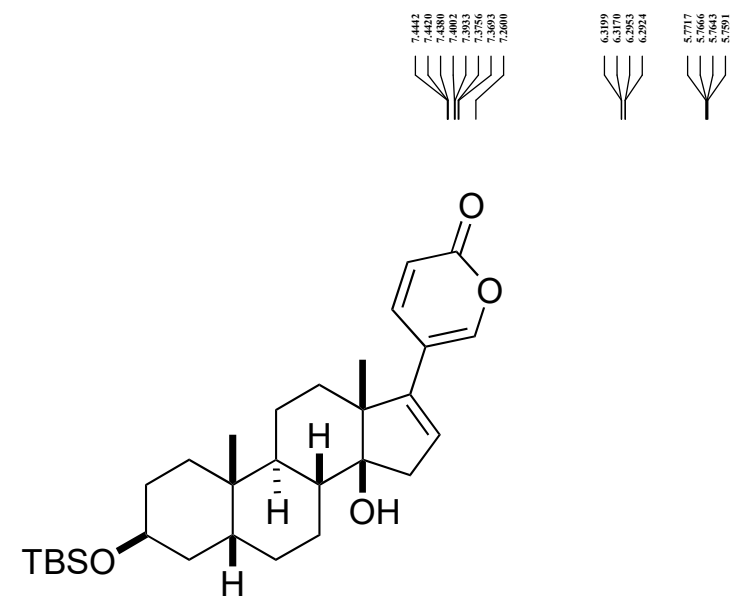

15

${ }^{1} \mathrm{H}$ NMR $\left(400 \mathrm{MHz}, \mathrm{CDCl}_{3}\right)$

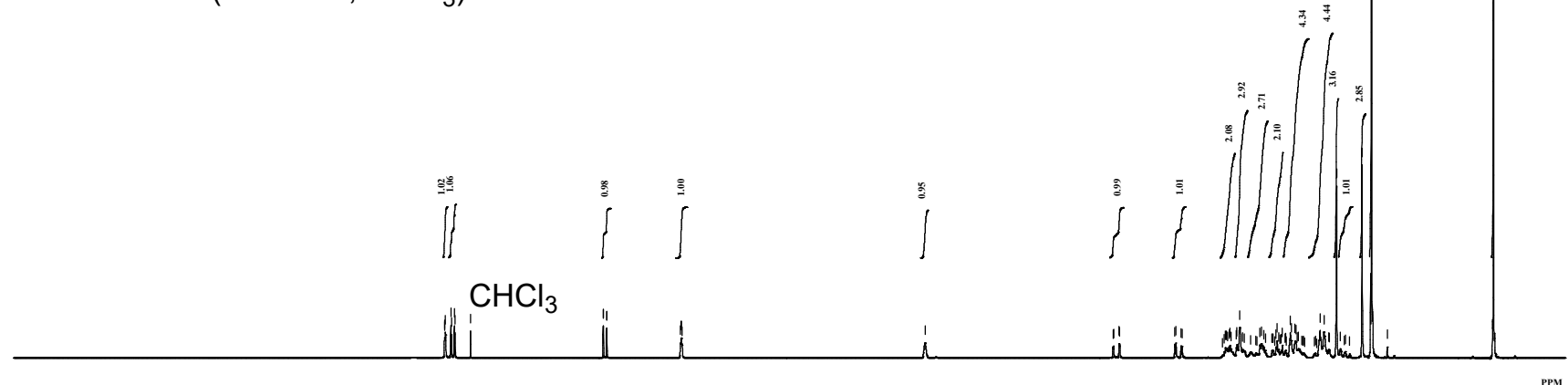

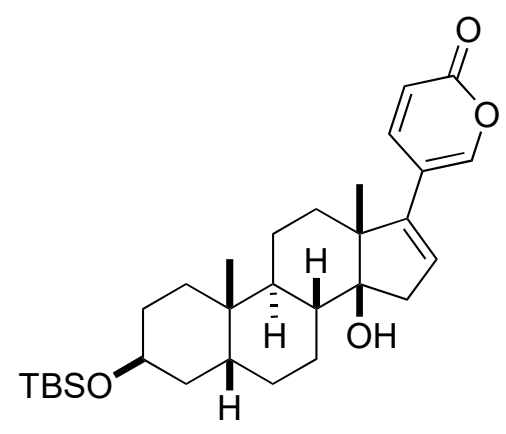

15

${ }^{13} \mathrm{C}\left\{{ }^{1} \mathrm{H}\right\}$ NMR $\left(100 \mathrm{MHz}, \mathrm{CDCl}_{3}\right)$

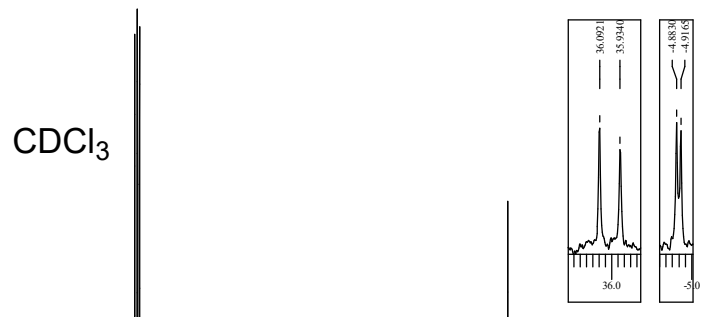

(1) (In

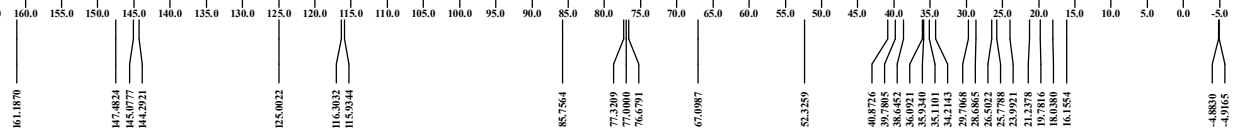




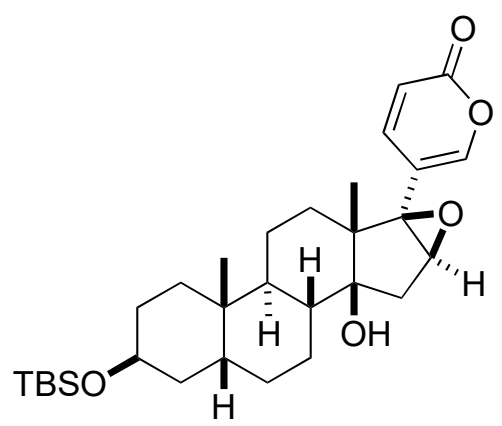

6

${ }^{1} \mathrm{H}$ NMR $\left(400 \mathrm{MHz}, \mathrm{C}_{6} \mathrm{D}_{6}\right)$
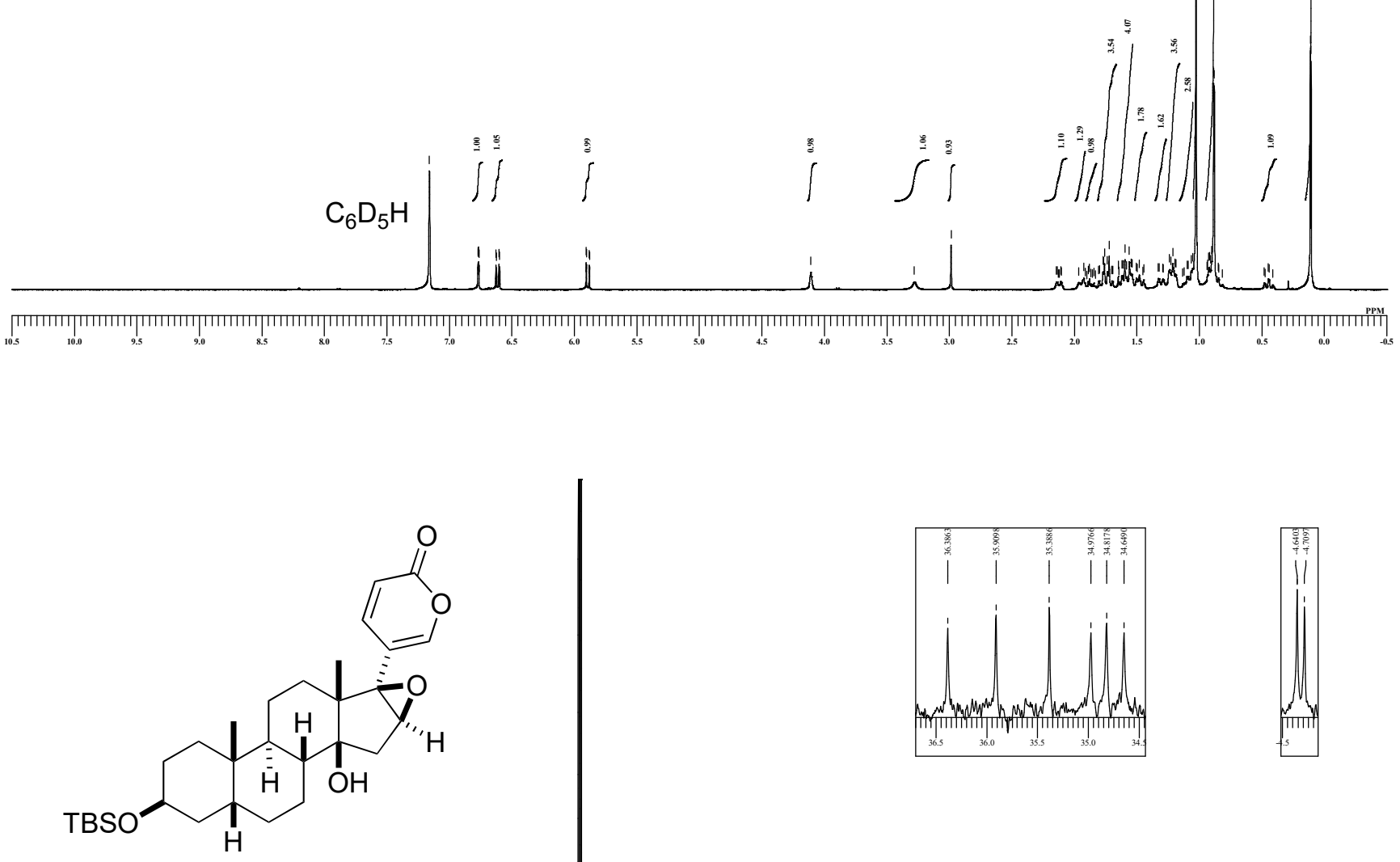

6

${ }^{13} \mathrm{C}\left\{{ }^{1} \mathrm{H}\right\}$ NMR $\left(100 \mathrm{MHz}, \mathrm{C}_{6} \mathrm{D}_{6}\right)$
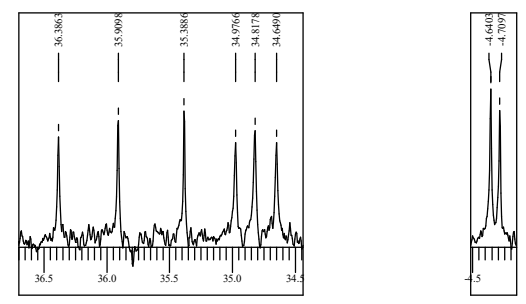

$\mathrm{C}_{6} \mathrm{D}_{6}$

m

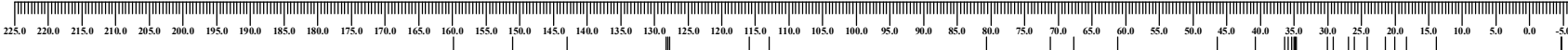

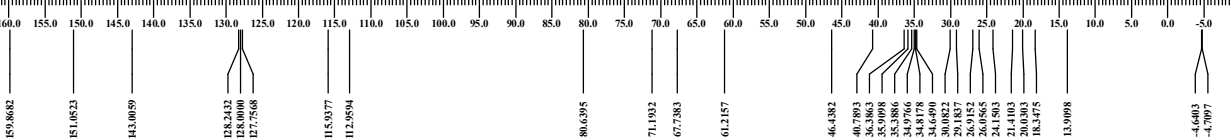



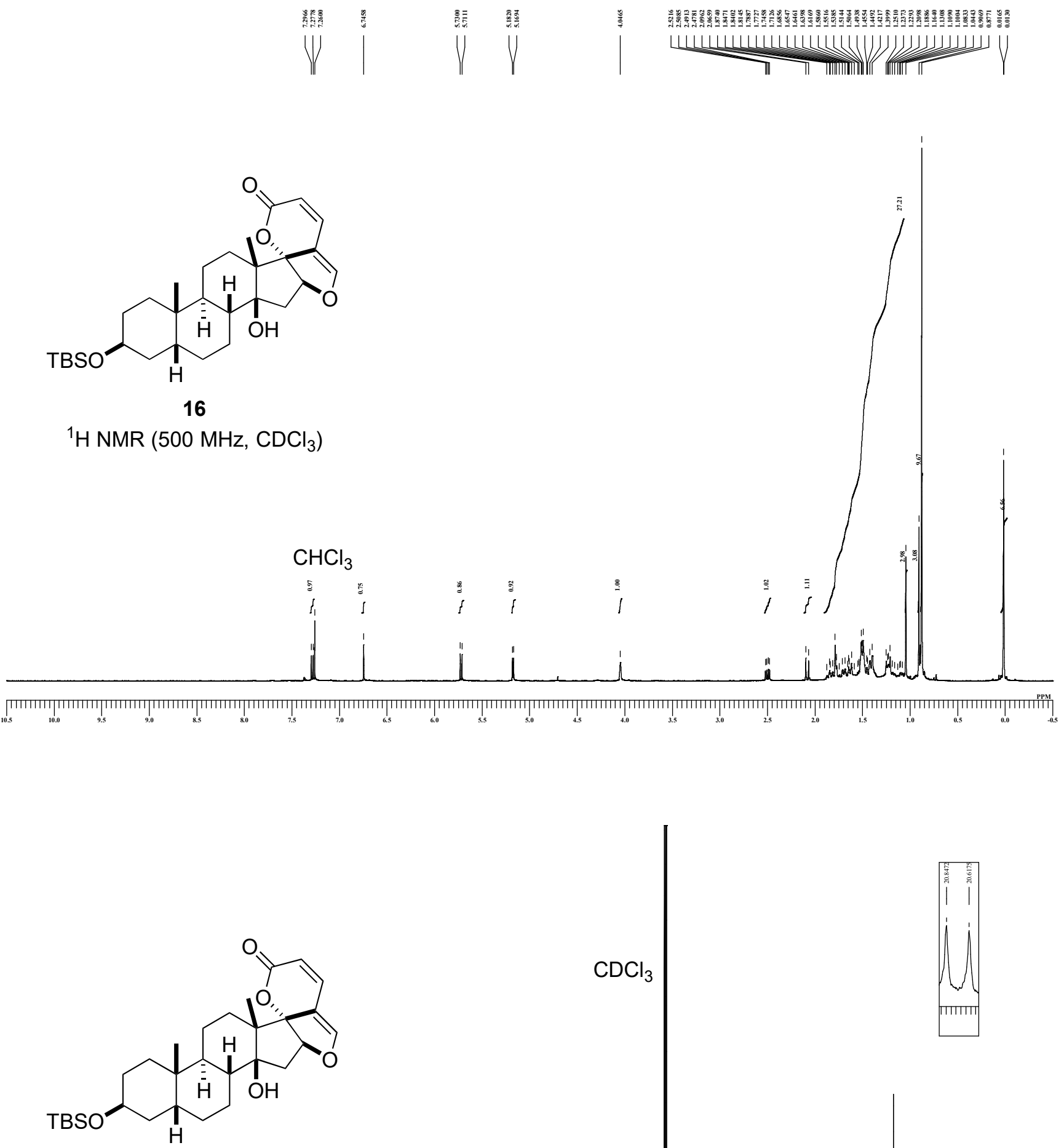

16

${ }^{13} \mathrm{C}\left\{{ }^{1} \mathrm{H}\right\} \operatorname{NMR}\left(125 \mathrm{MHz}, \mathrm{CDCl}_{3}\right)$ 

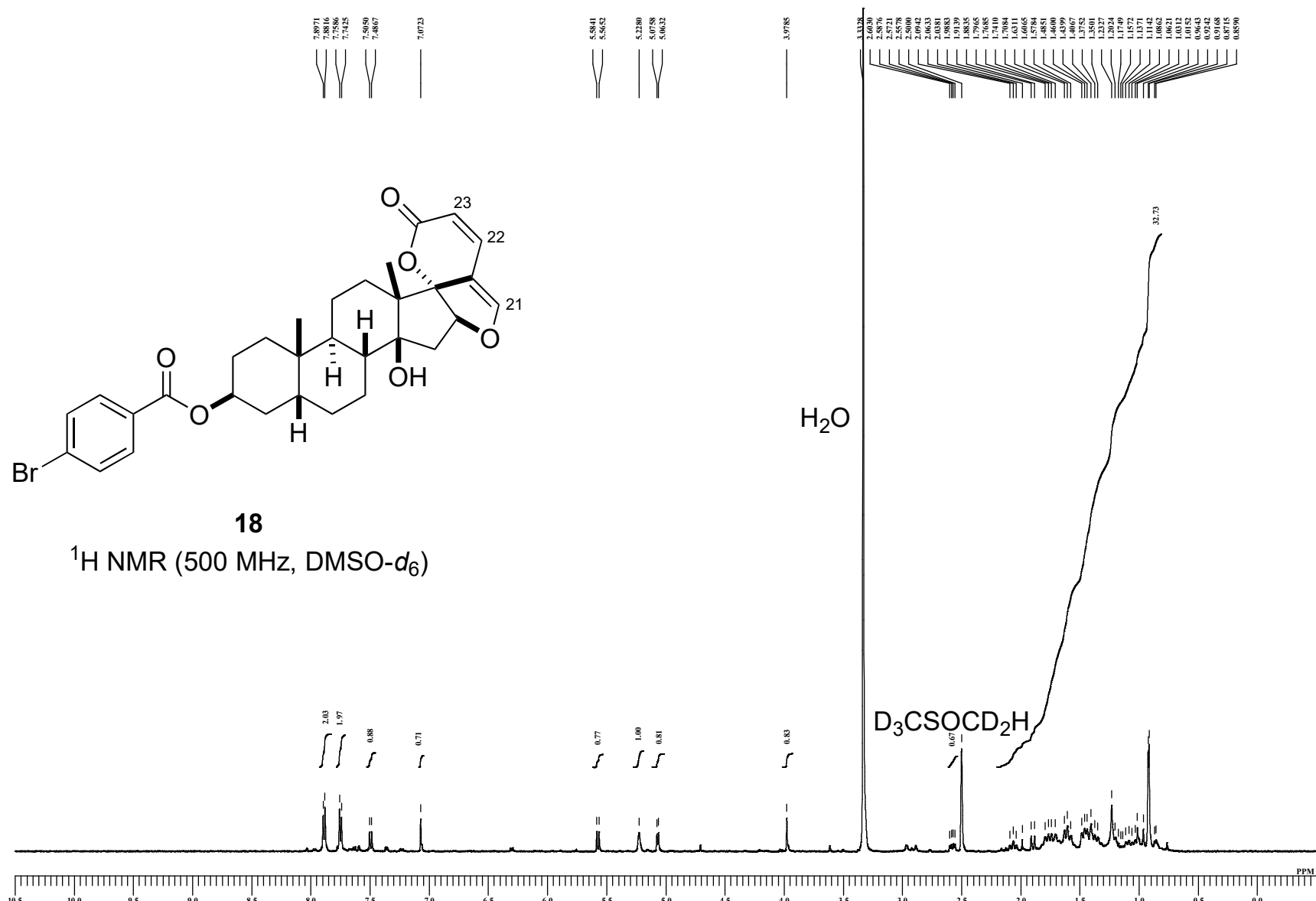

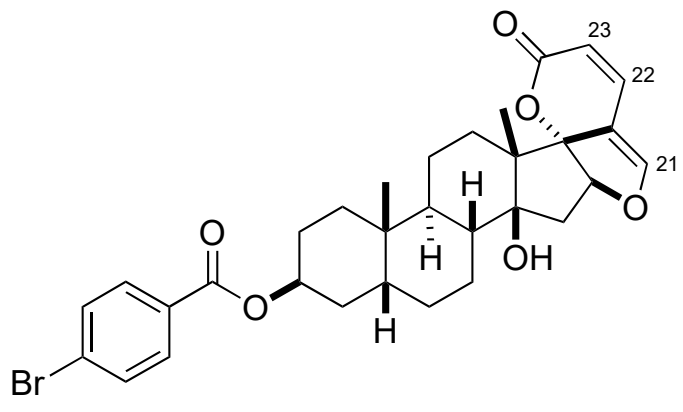

18

${ }^{13} \mathrm{C}\left\{{ }^{1} \mathrm{H}\right\}$ NMR $\left(100 \mathrm{MHz}\right.$, DMSO- $\left.d_{6}\right)$

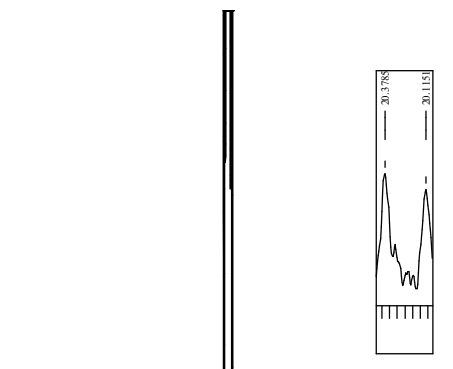

$\mathrm{D}_{3} \mathrm{CSOCD}_{3}$

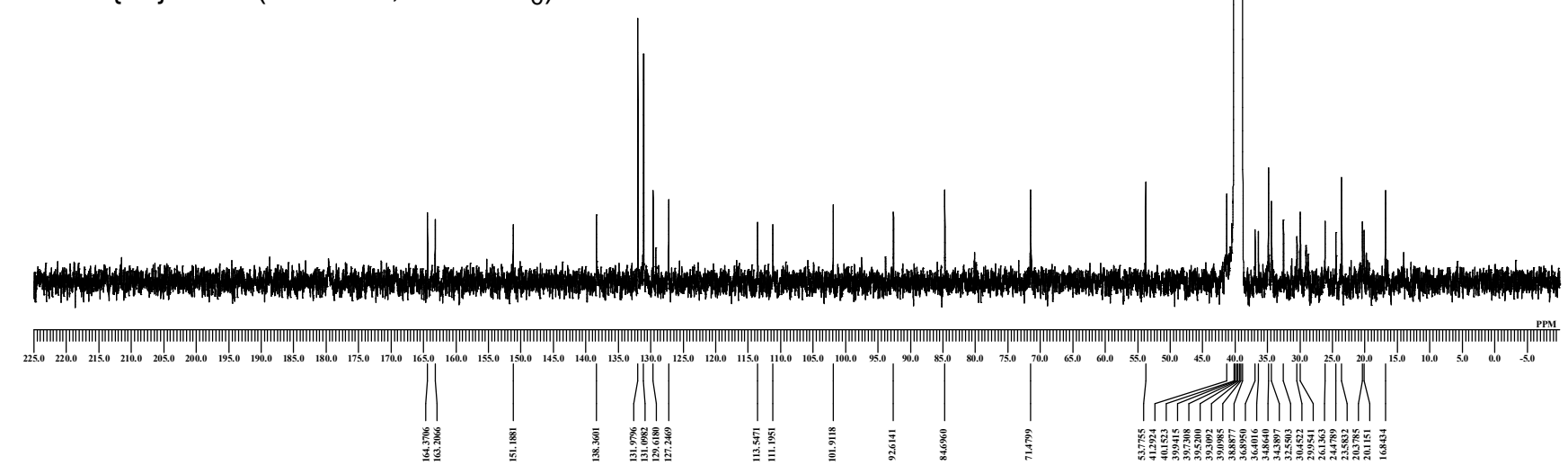




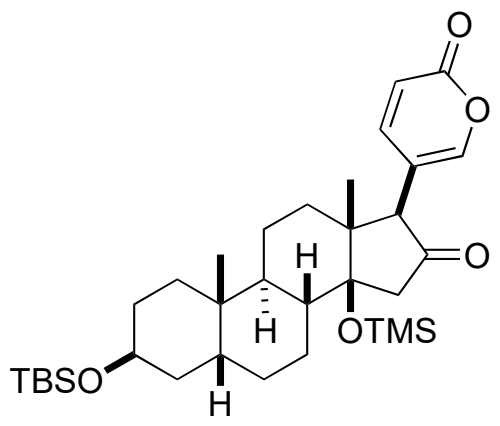

19

${ }^{1} \mathrm{H}$ NMR $\left(400 \mathrm{MHz}, \mathrm{C}_{6} \mathrm{D}_{6}\right)$

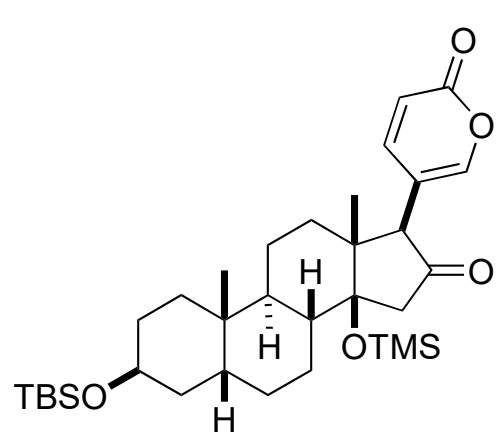

19

${ }^{13} \mathrm{C}\left\{{ }^{1} \mathrm{H}\right\}$ NMR $\left(100 \mathrm{MHz}, \mathrm{C}_{6} \mathrm{D}_{6}\right)$

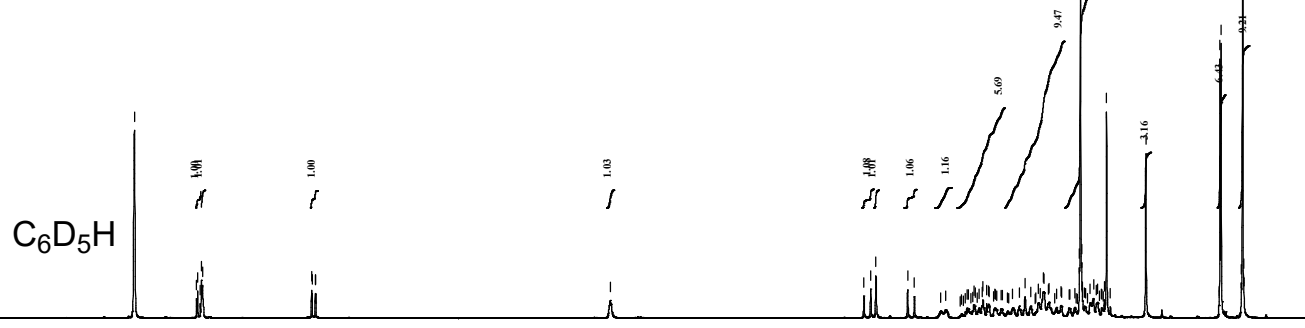

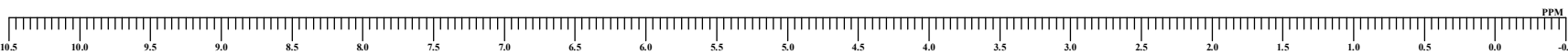

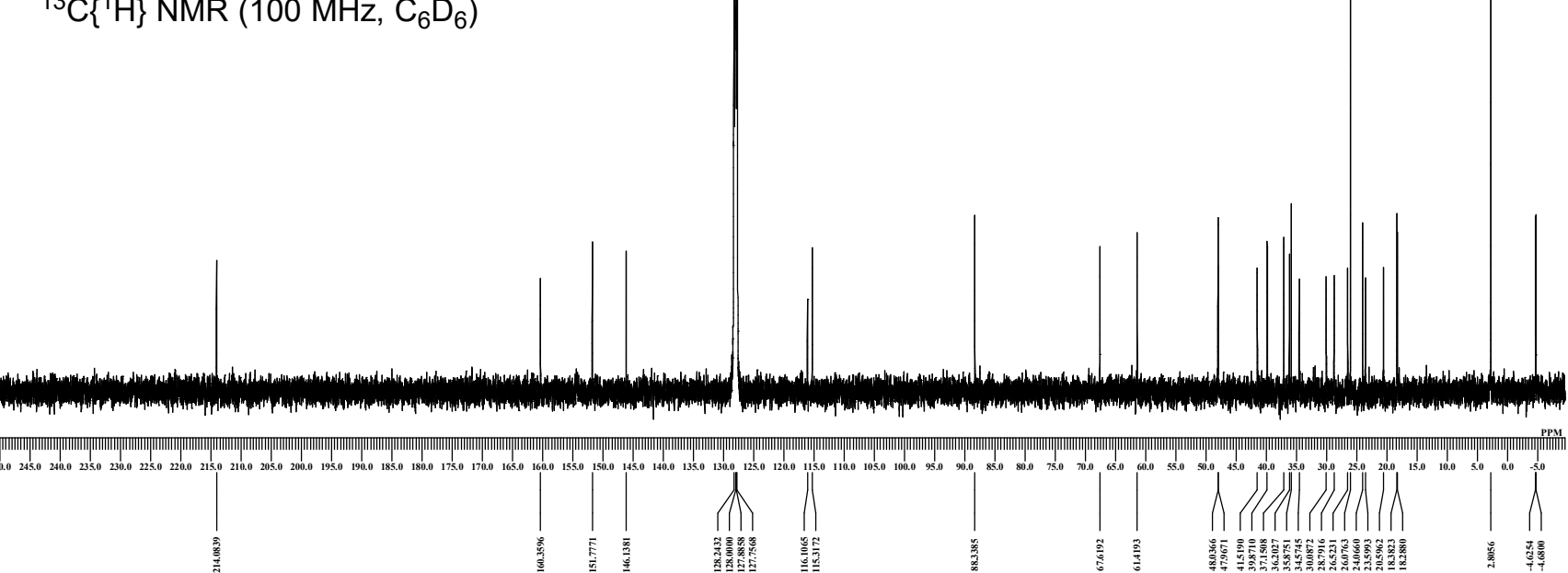



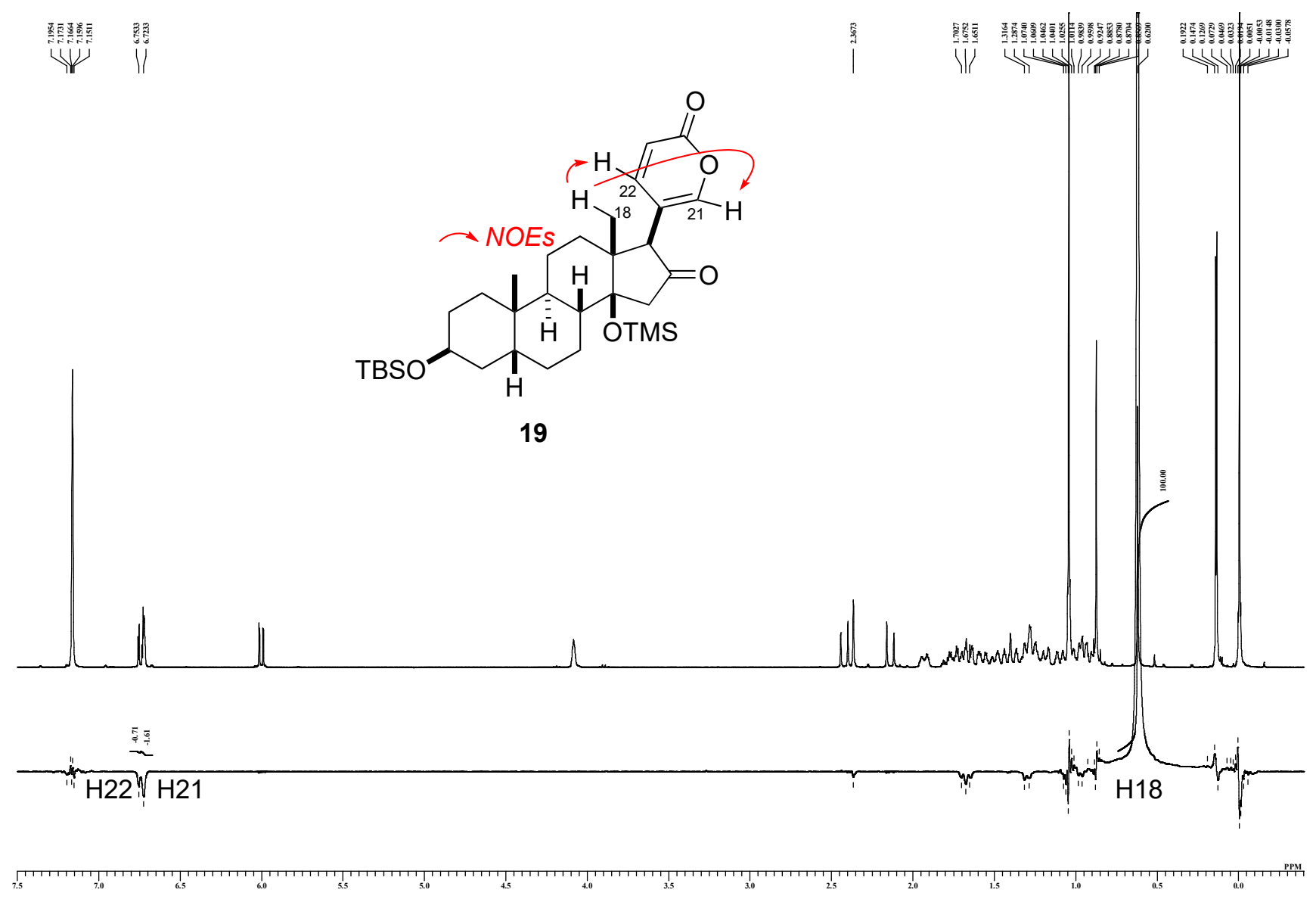

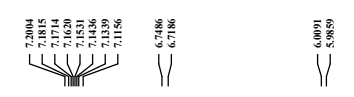

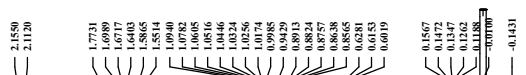

11 MYII

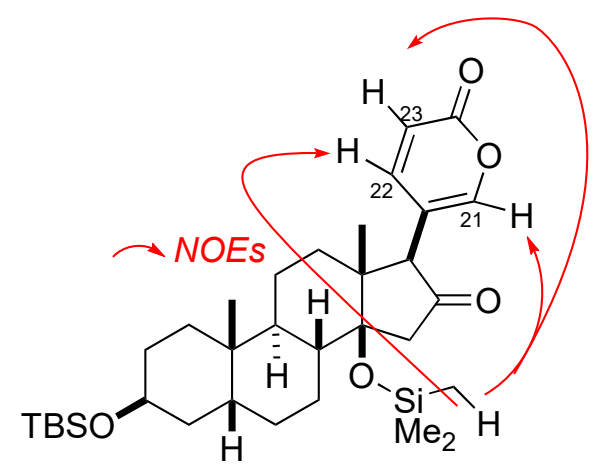

19

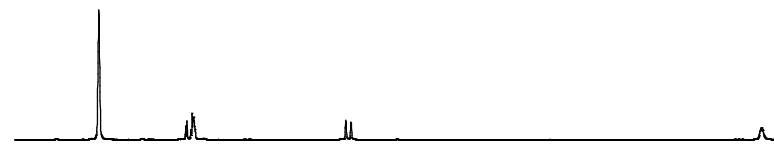

ul

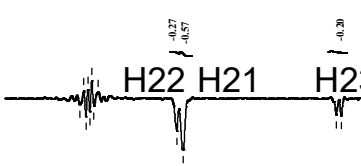

\section{$\stackrel{1}{2}$}




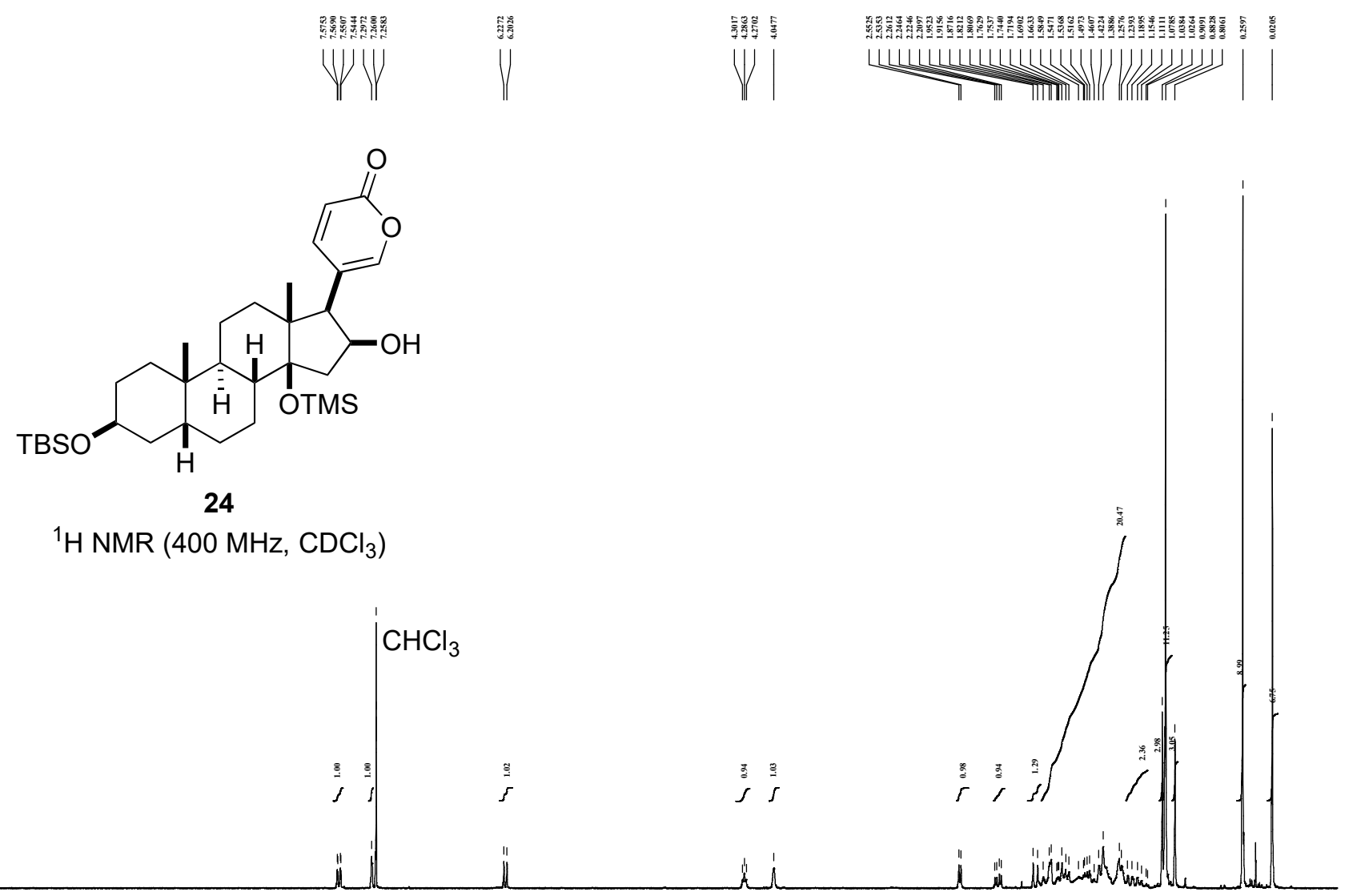

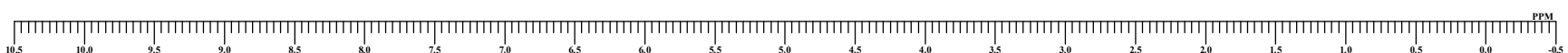
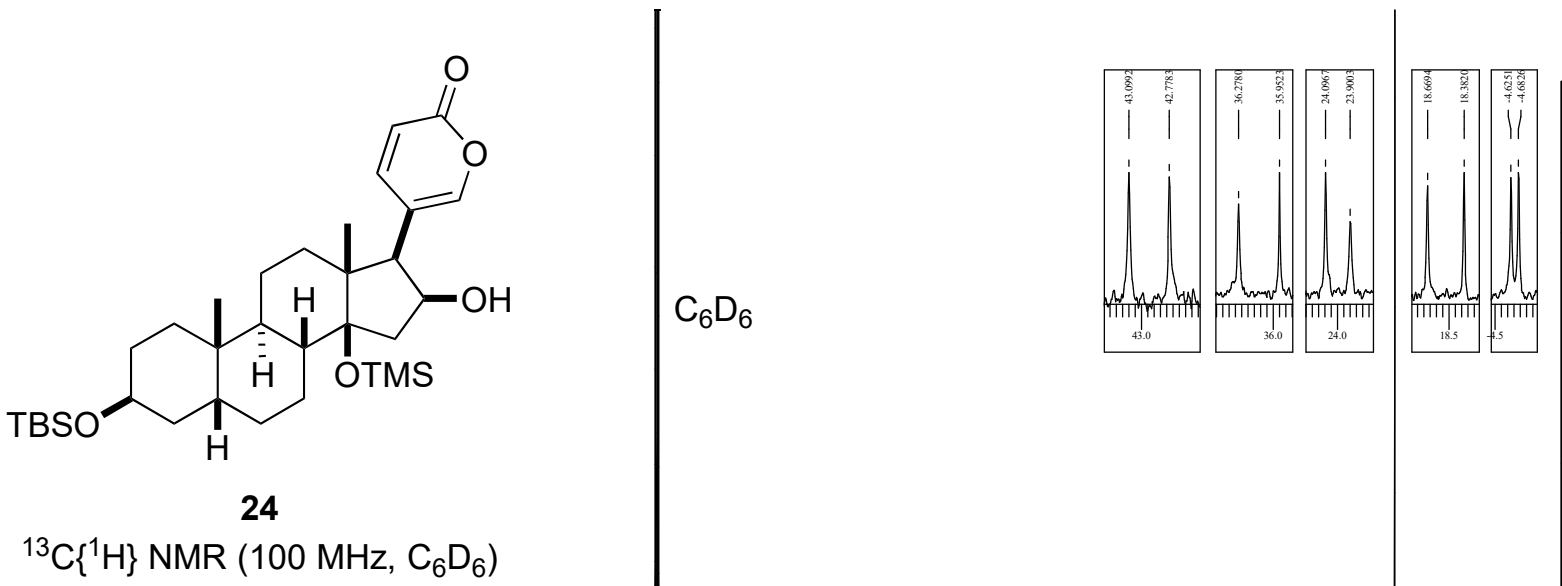

${ }^{13} \mathrm{C}\left\{{ }^{1} \mathrm{H}\right\}$ NMR $\left(100 \mathrm{MHz}, \mathrm{C}_{6} \mathrm{D}_{6}\right)$

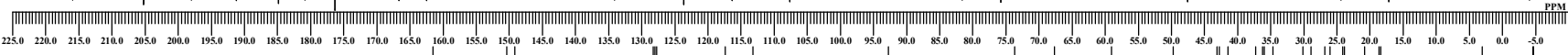

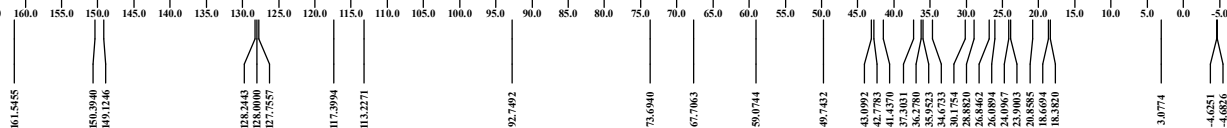




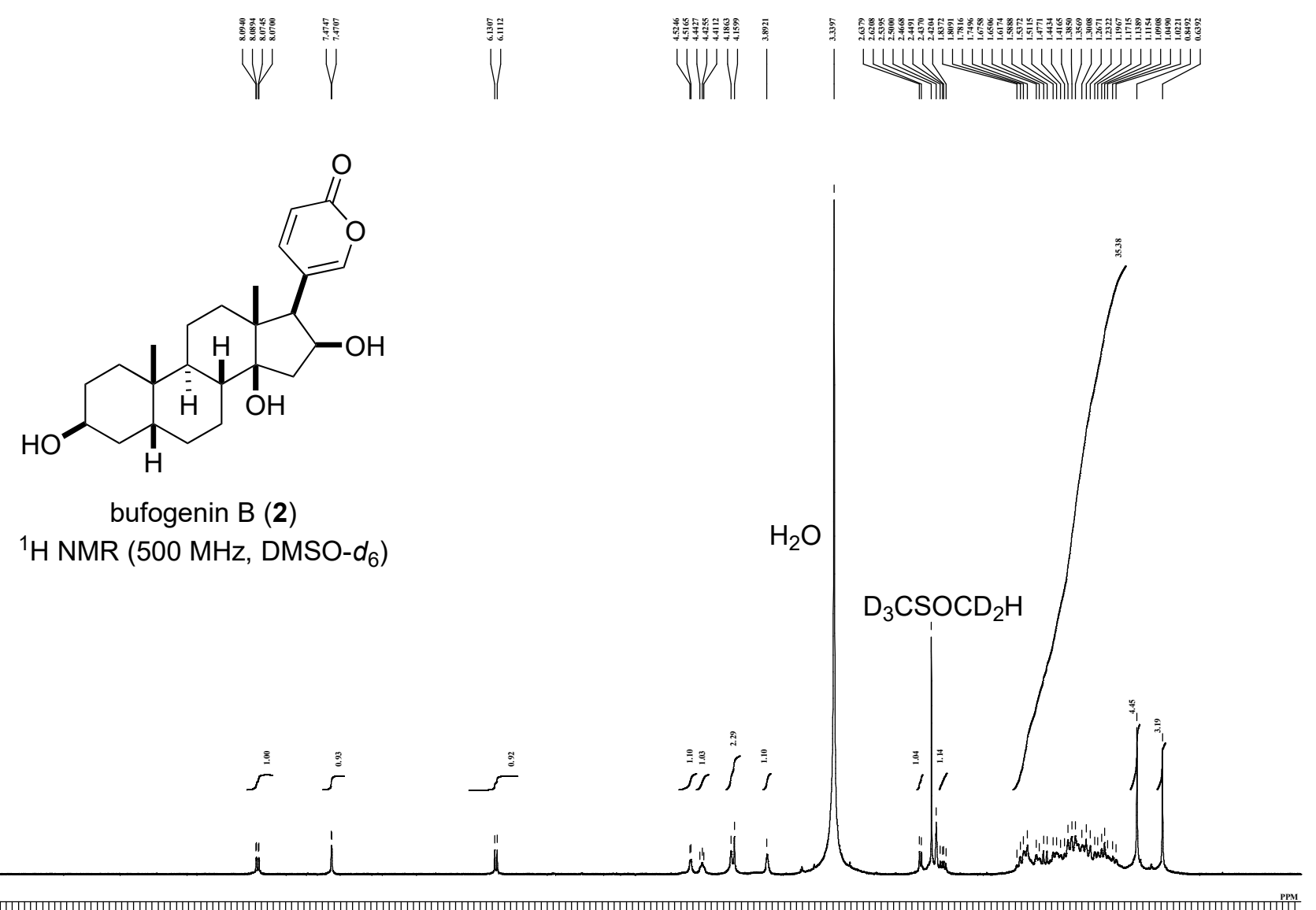

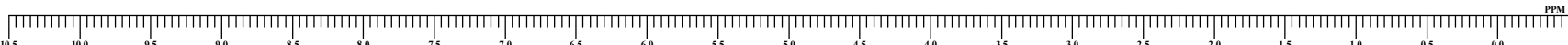
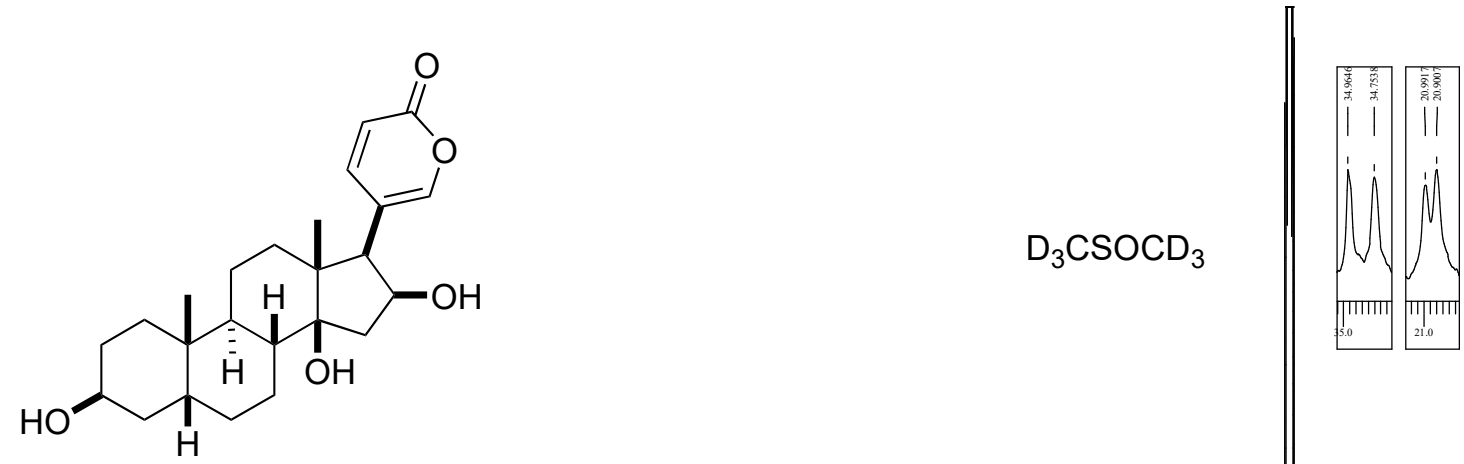

bufogenin $B(2)$

${ }^{13} \mathrm{C}\left\{{ }^{1} \mathrm{H}\right\}$ NMR $\left(100 \mathrm{MHz}\right.$, DMSO- $\left.d_{6}\right)$ 


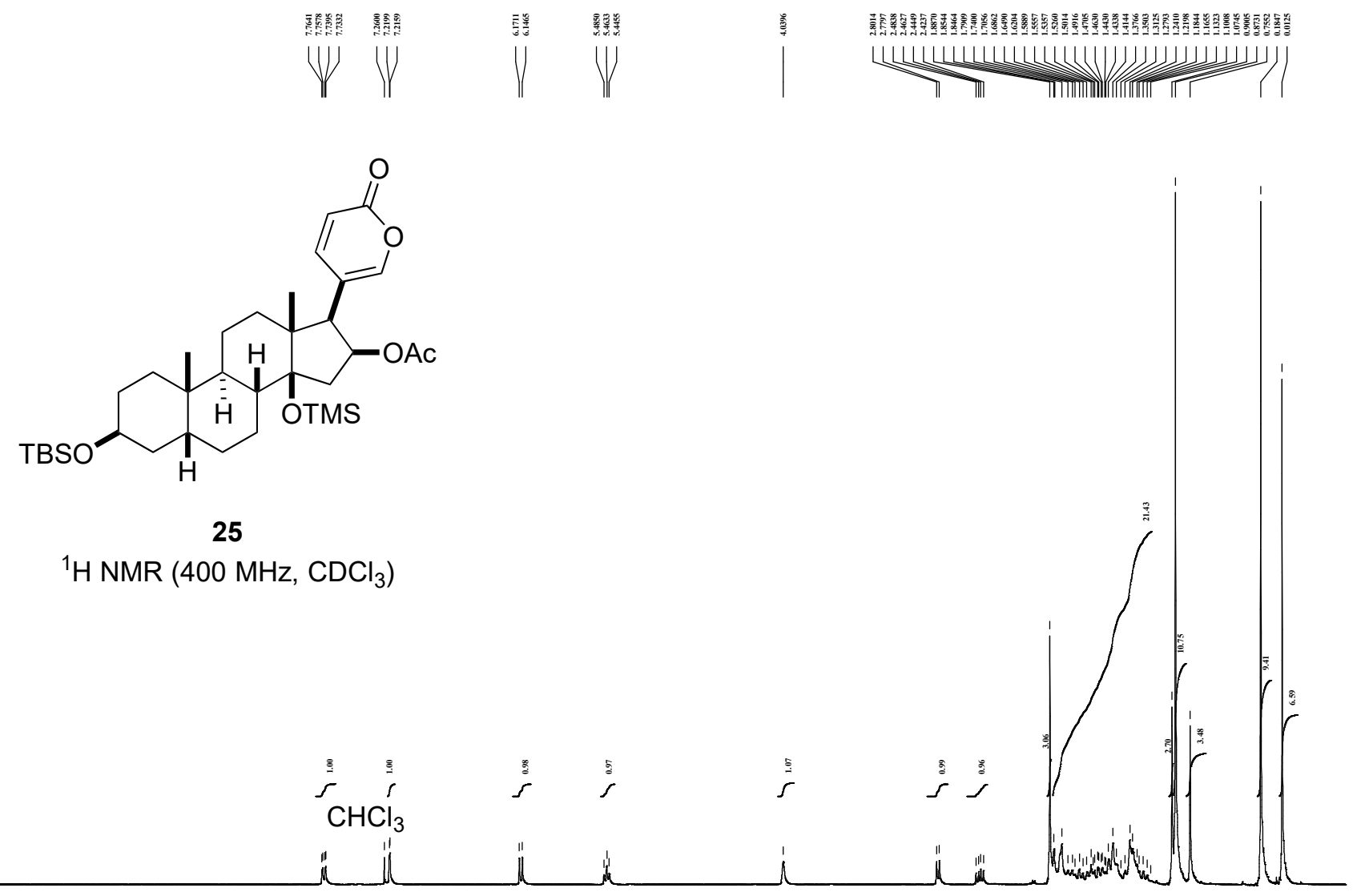

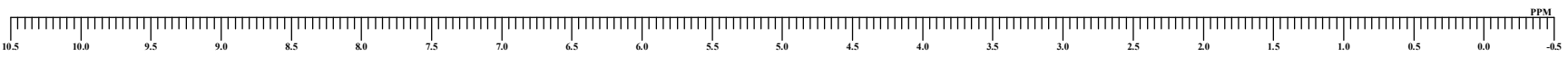
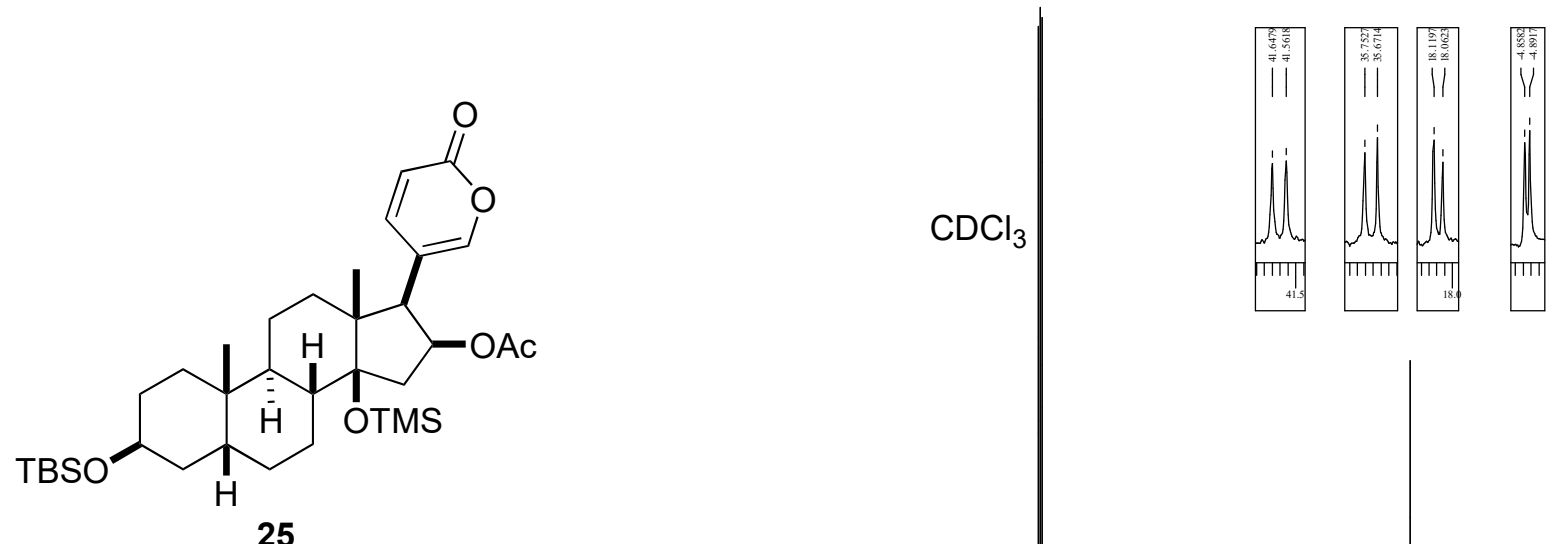

${ }^{13} \mathrm{C}\left\{{ }^{1} \mathrm{H}\right\}$ NMR $\left(125 \mathrm{MHz}, \mathrm{CDCl}_{3}\right)$

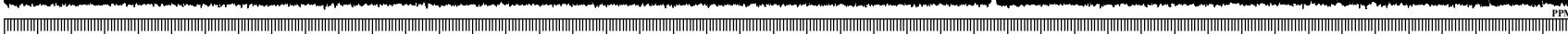

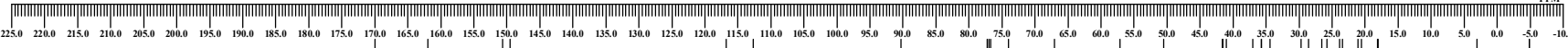




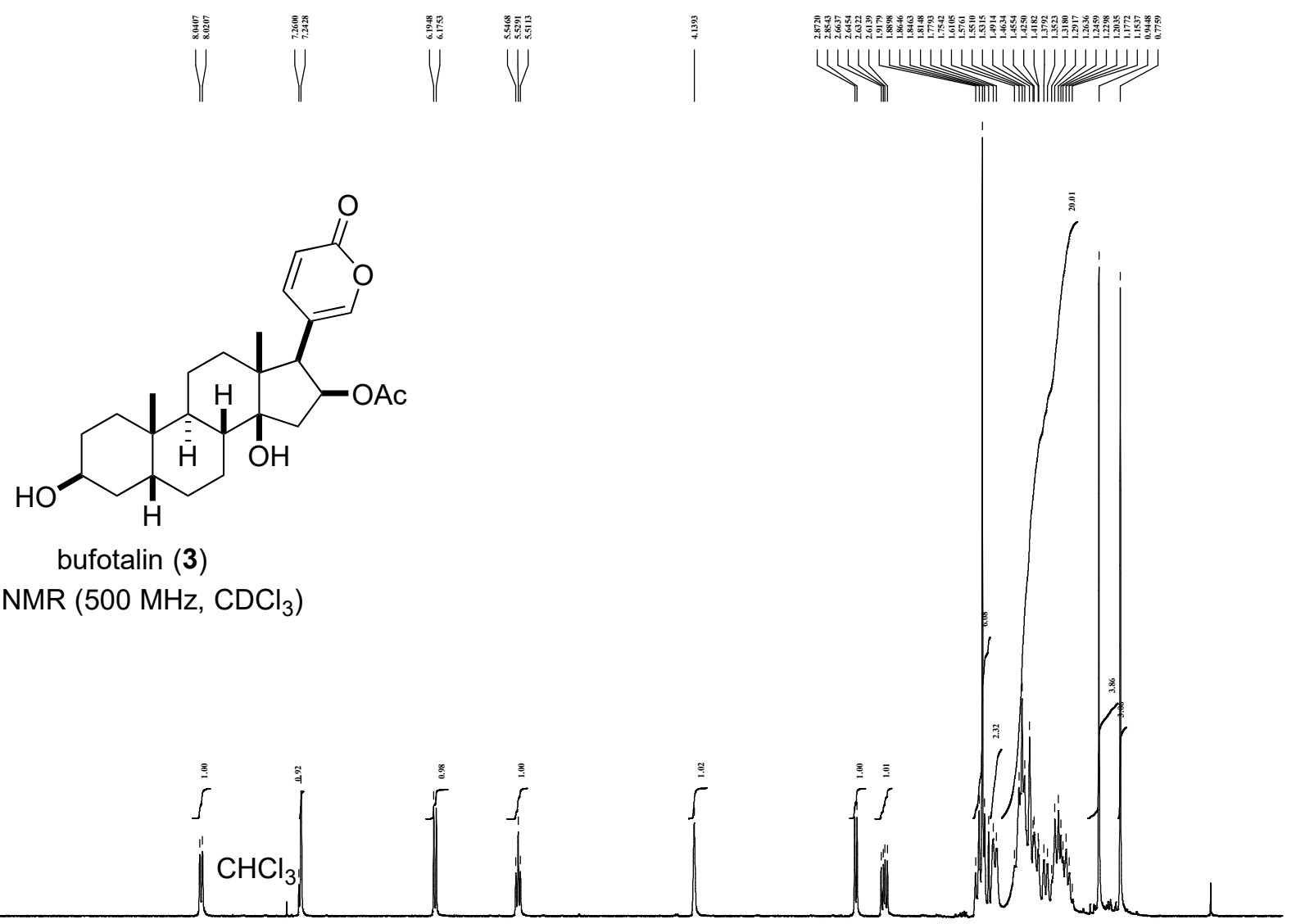

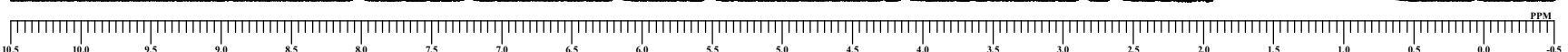

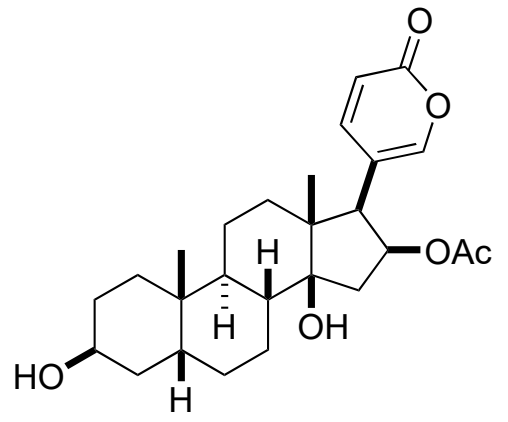

bufotalin (3)

${ }^{13} \mathrm{C}\left\{{ }^{1} \mathrm{H}\right\}$ NMR $\left(125 \mathrm{MHz}, \mathrm{CDCl}_{3}\right)$

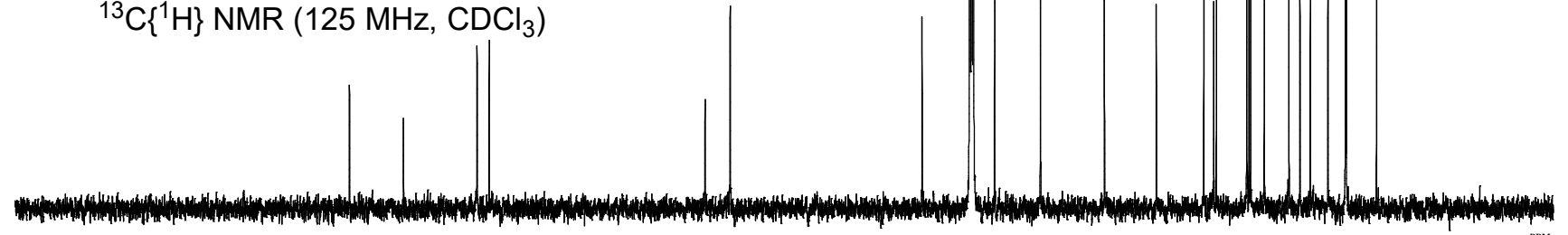

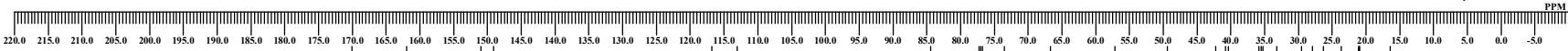

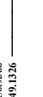




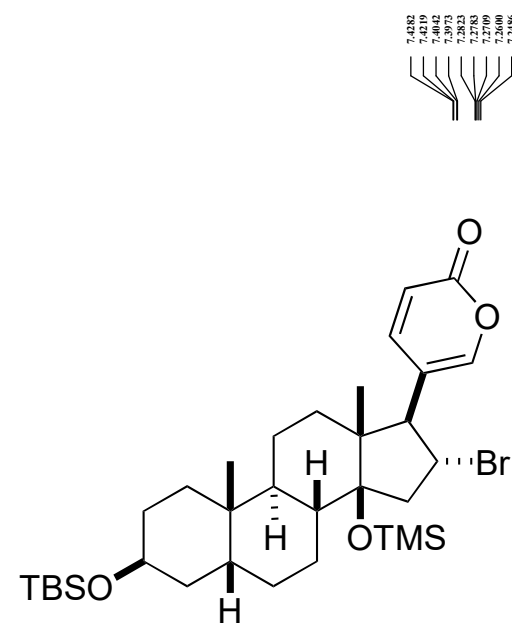

26

${ }^{1} \mathrm{H}$ NMR $\left(400 \mathrm{MHz}, \mathrm{CDCl}_{3}\right)$
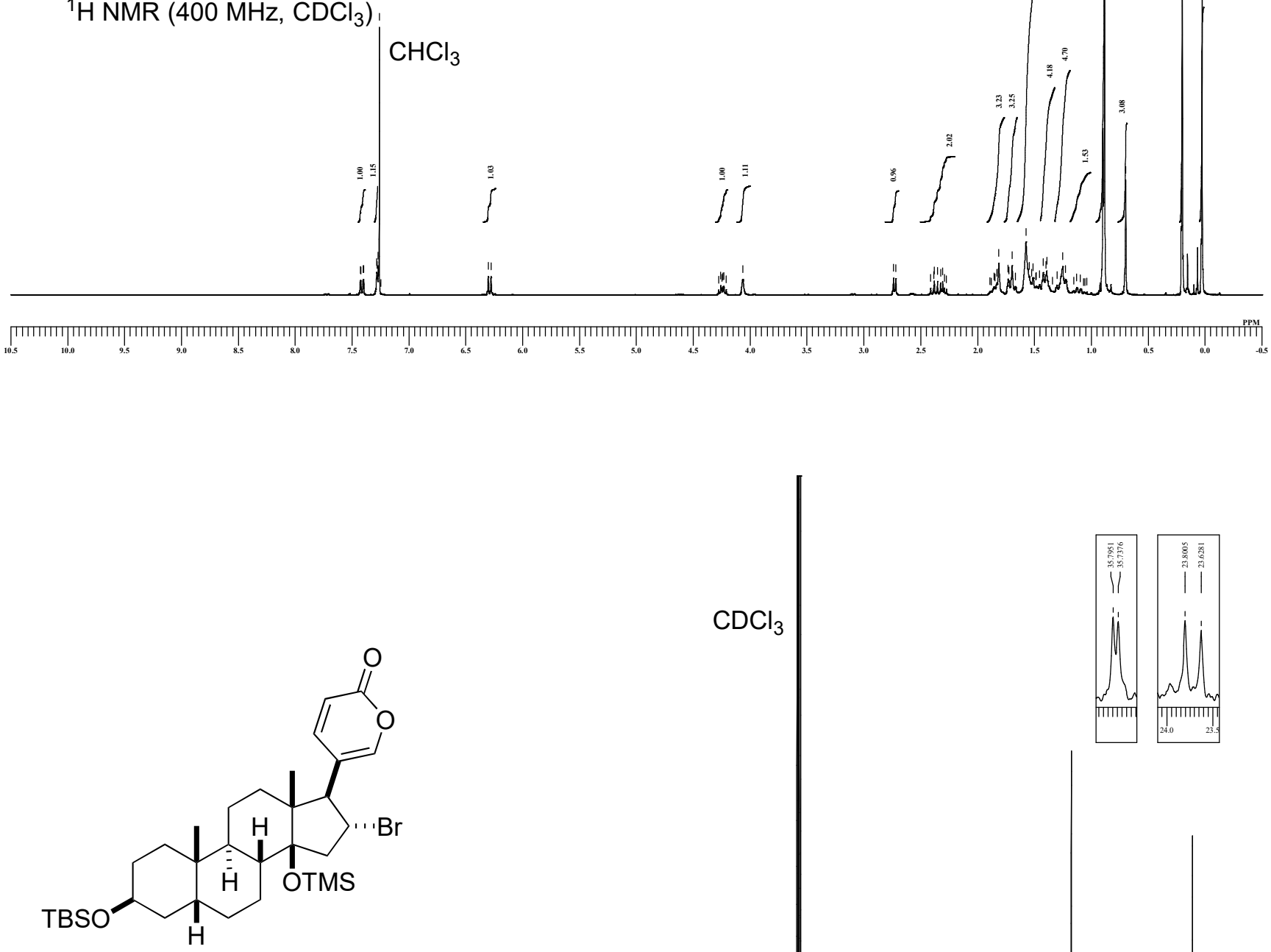

26

${ }^{13} \mathrm{C}\left\{{ }^{1} \mathrm{H}\right\} \operatorname{NMR}\left(100 \mathrm{MHz}, \mathrm{CDCl}_{3}\right)$

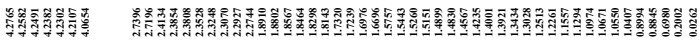

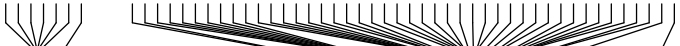

( 

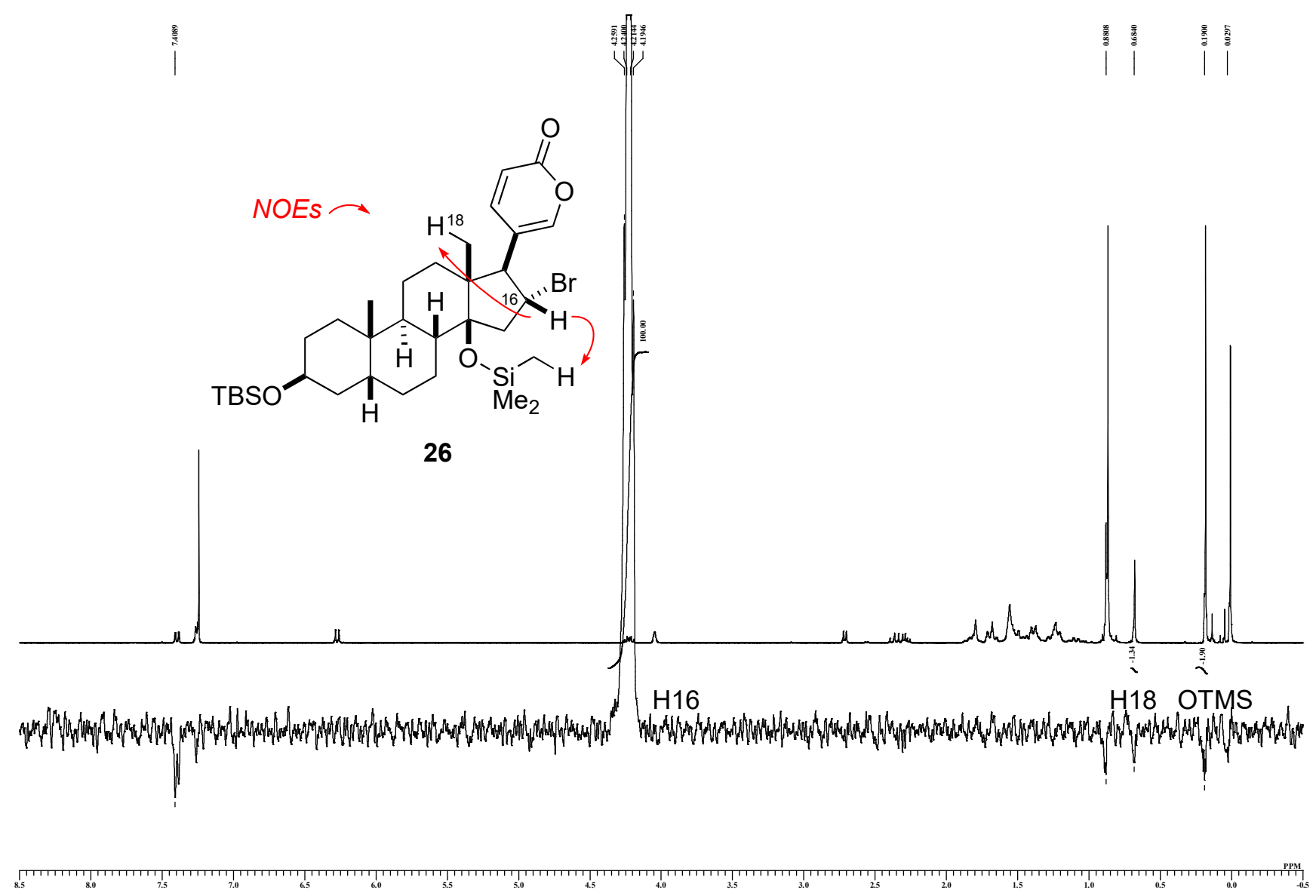


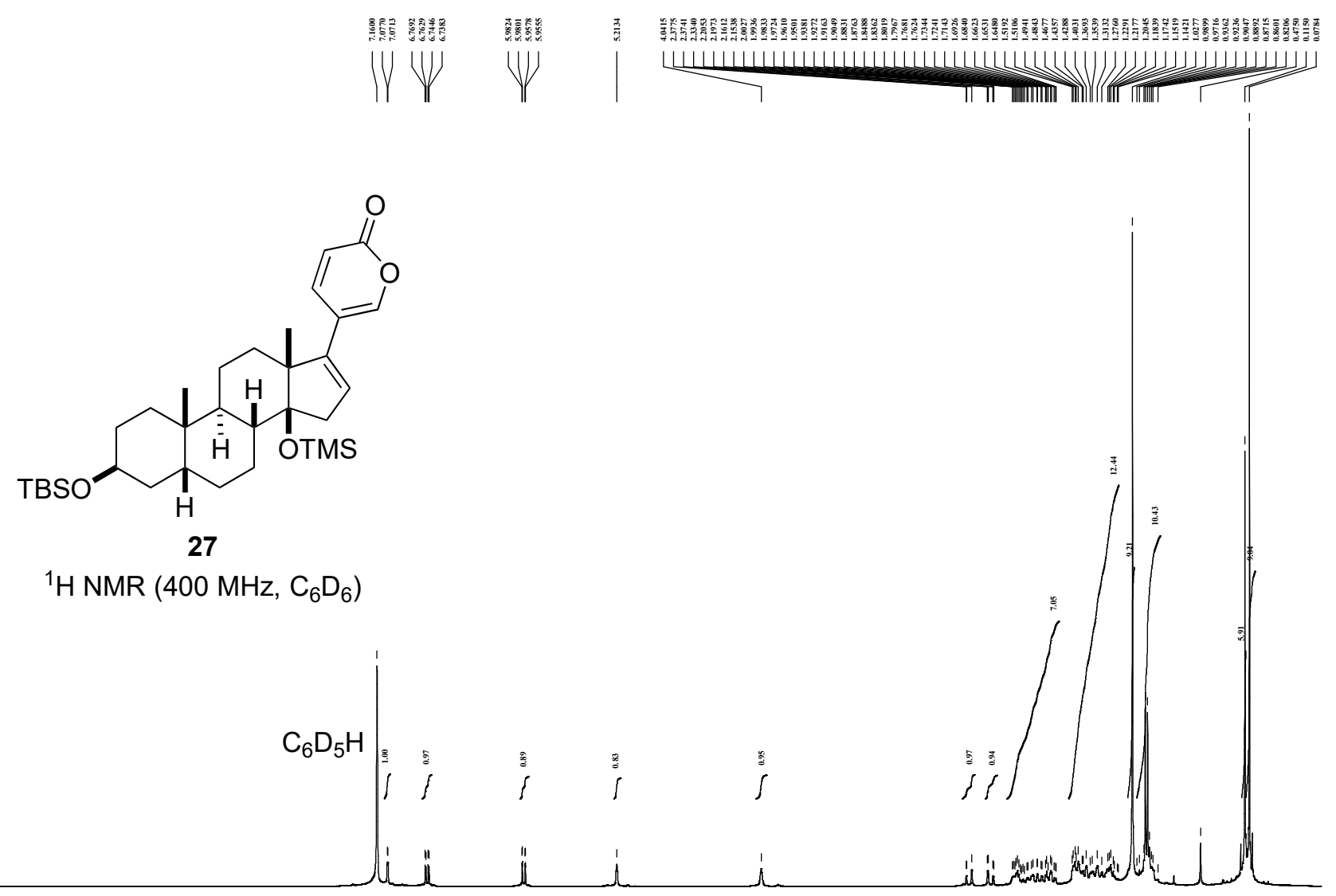

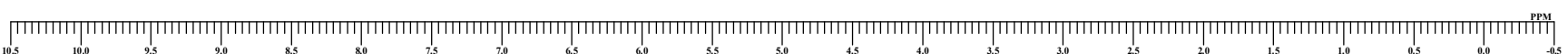

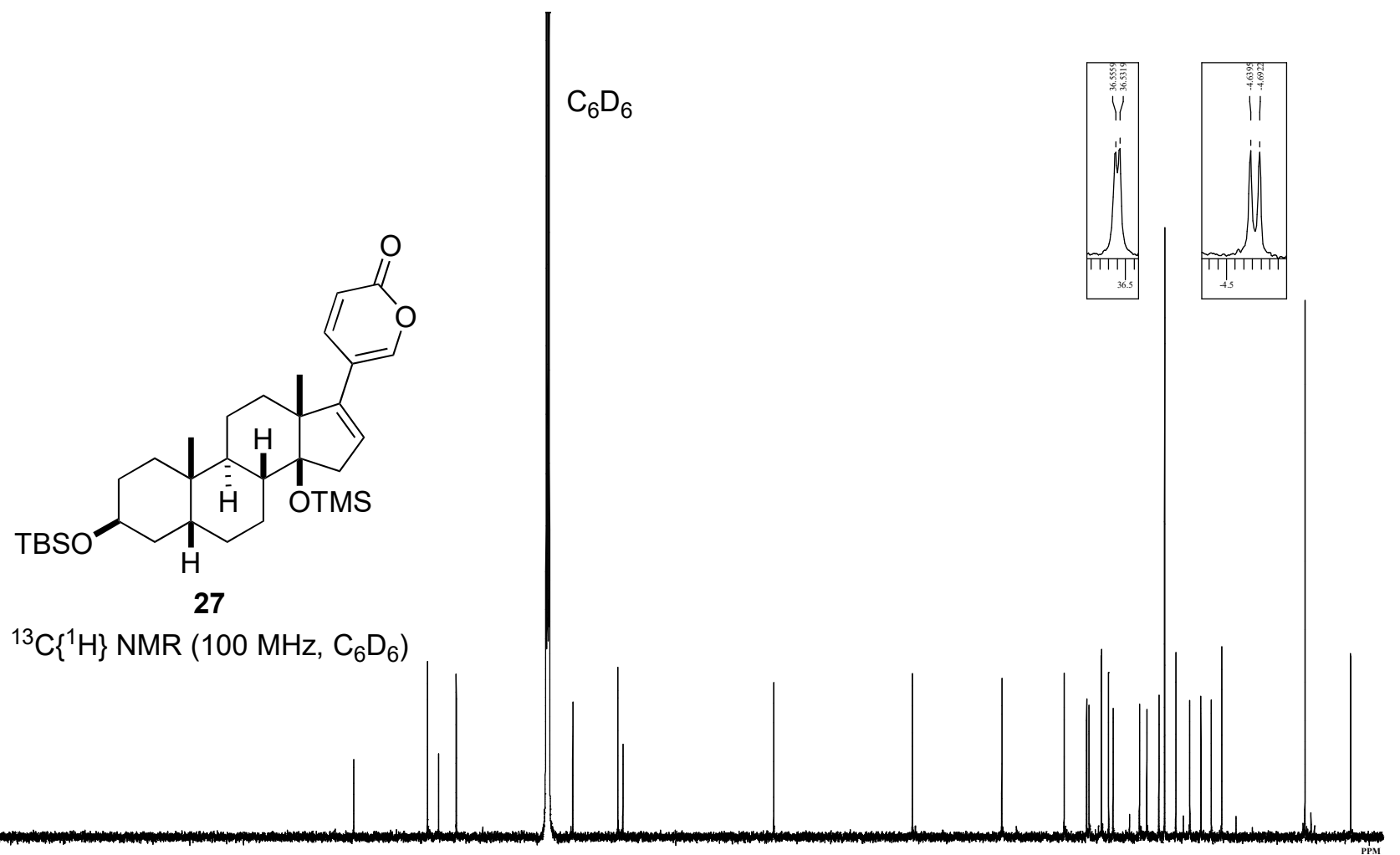

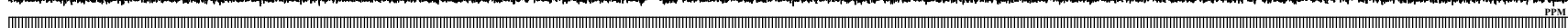

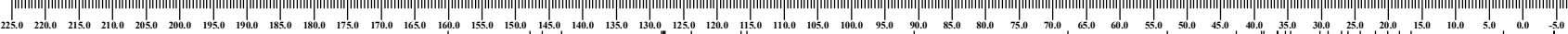

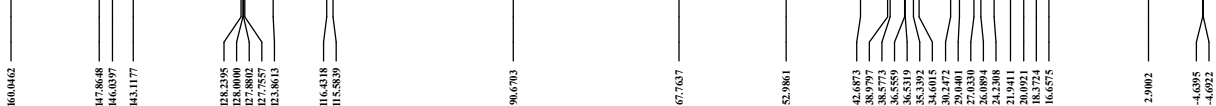




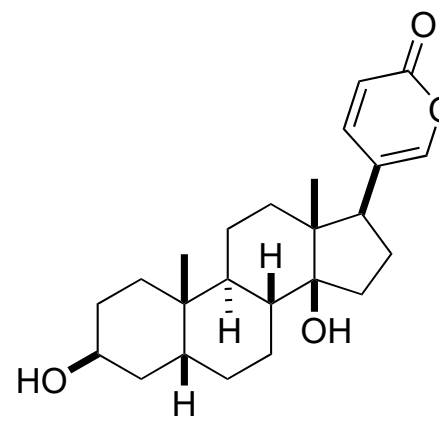

bufalin (1)

${ }^{1} \mathrm{H}$ NMR $\left(400 \mathrm{MHz}, \mathrm{CDCl}_{3}\right)$
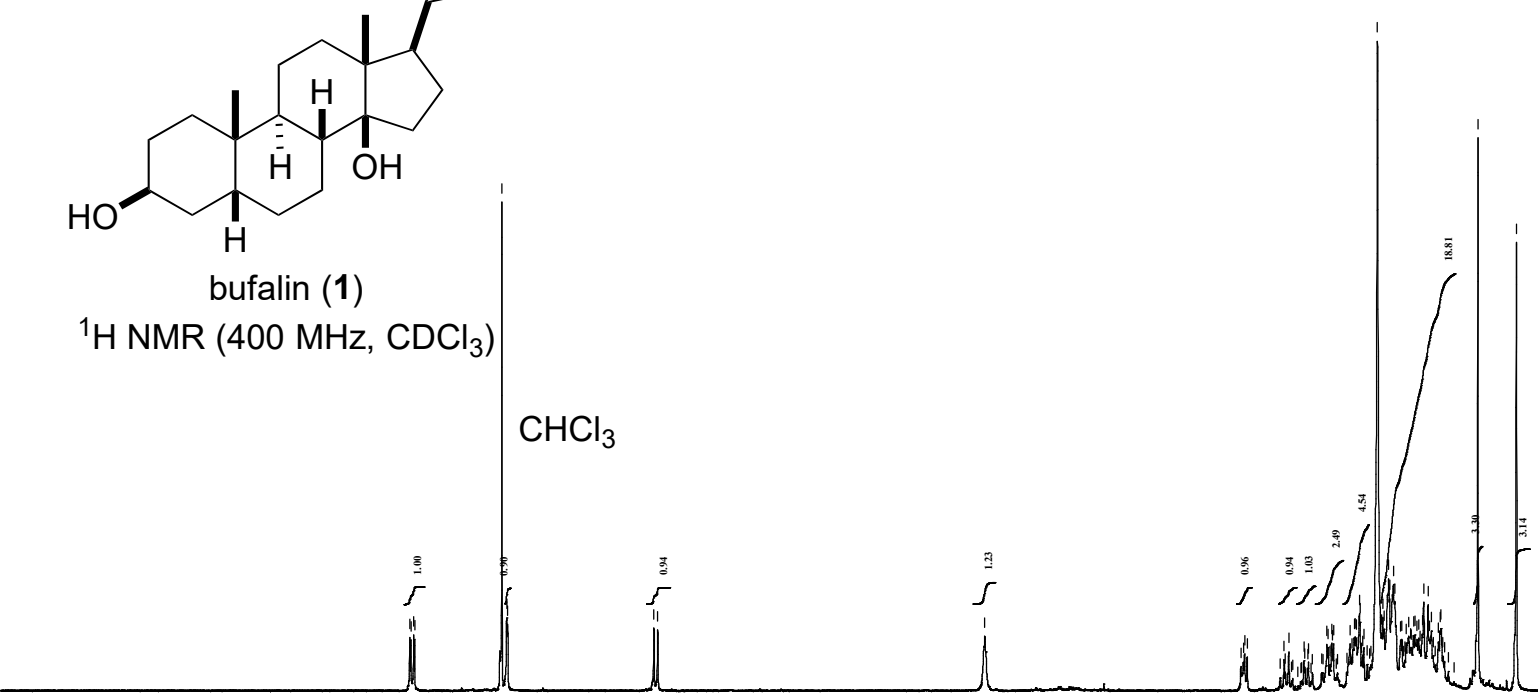

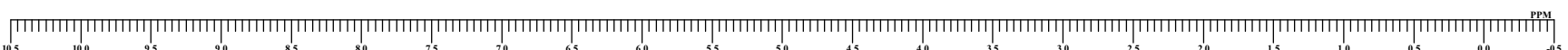

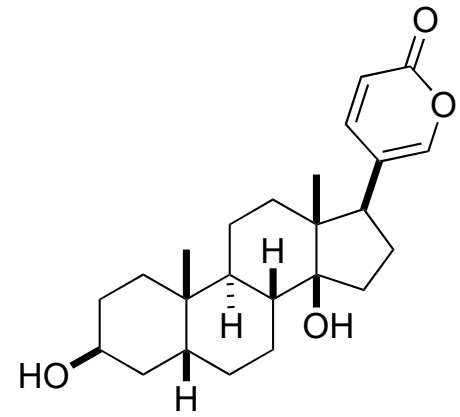

bufalin (1)

${ }^{13} \mathrm{C}\left\{{ }^{1} \mathrm{H}\right\} \mathrm{NMR}\left(100 \mathrm{MHz}, \mathrm{CDCl}_{3}\right)$
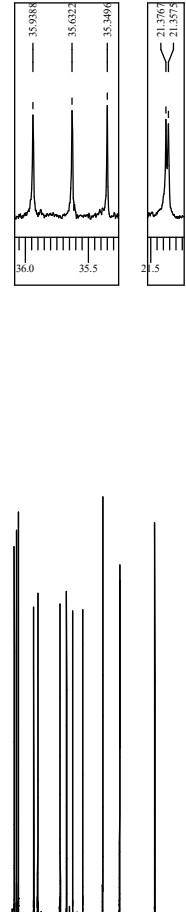

-

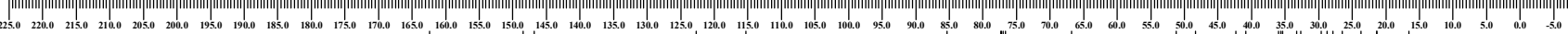



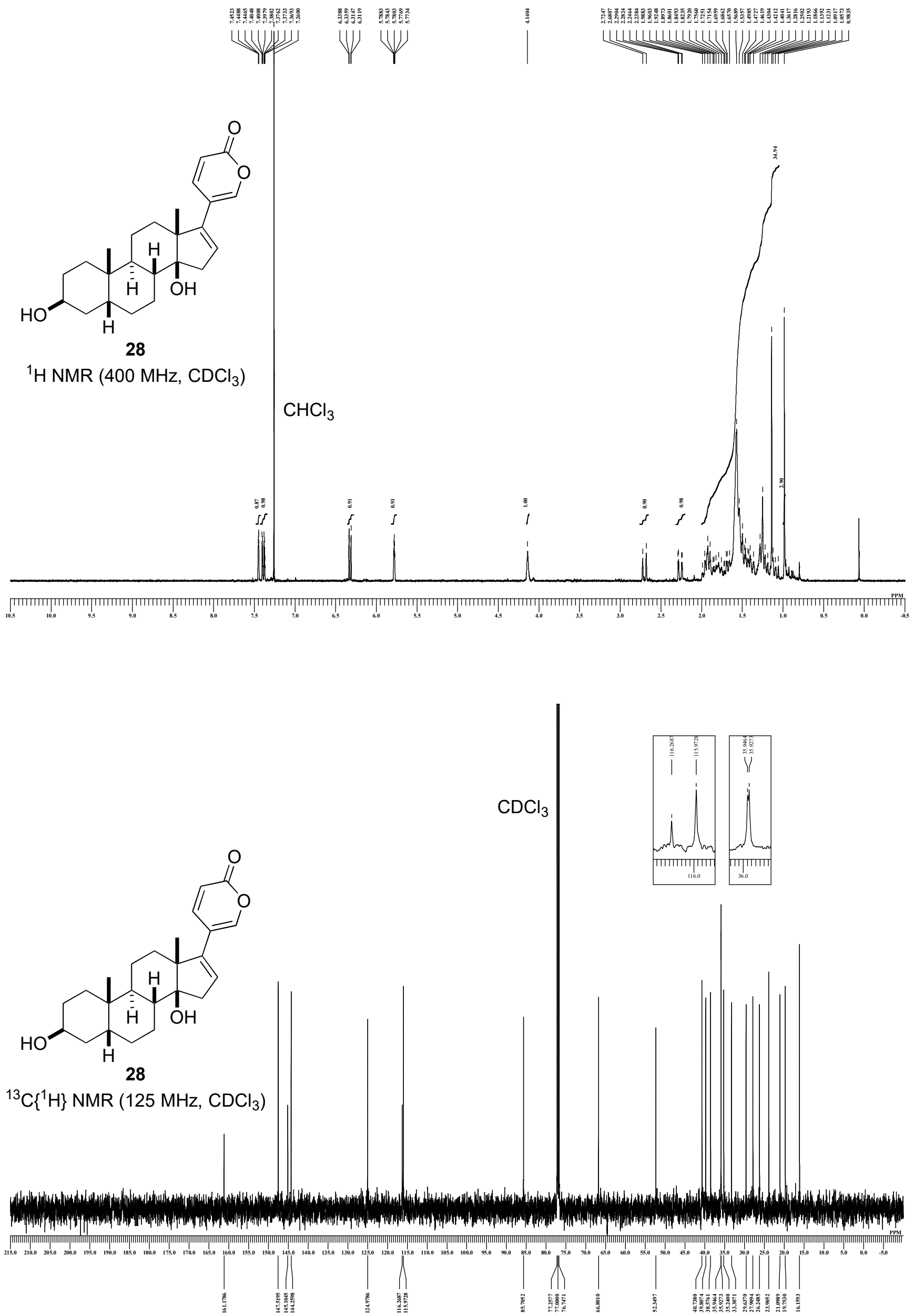
<smiles>O=C(O)CCCCCCC(=O)OBr</smiles>

S7

${ }^{1} \mathrm{H}$ NMR (500 MHz, $\mathrm{CDCl}_{3}$ )

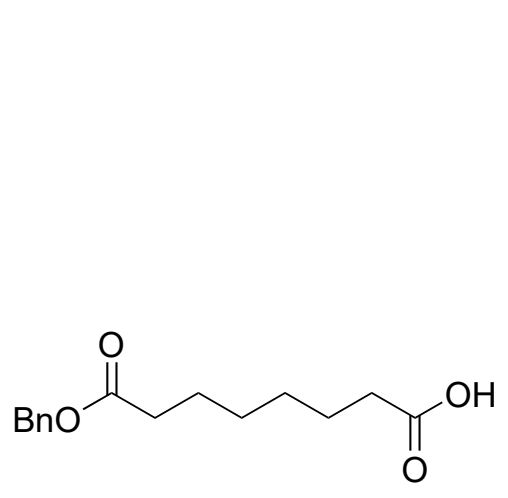
$\mathrm{CHCl}_{3}$

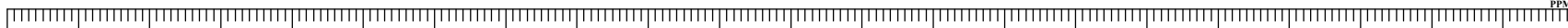

S7

${ }^{13} \mathrm{C}\left\{{ }^{1} \mathrm{H}\right\} \operatorname{NMR}\left(125 \mathrm{MHz}, \mathrm{CDCl}_{3}\right)$
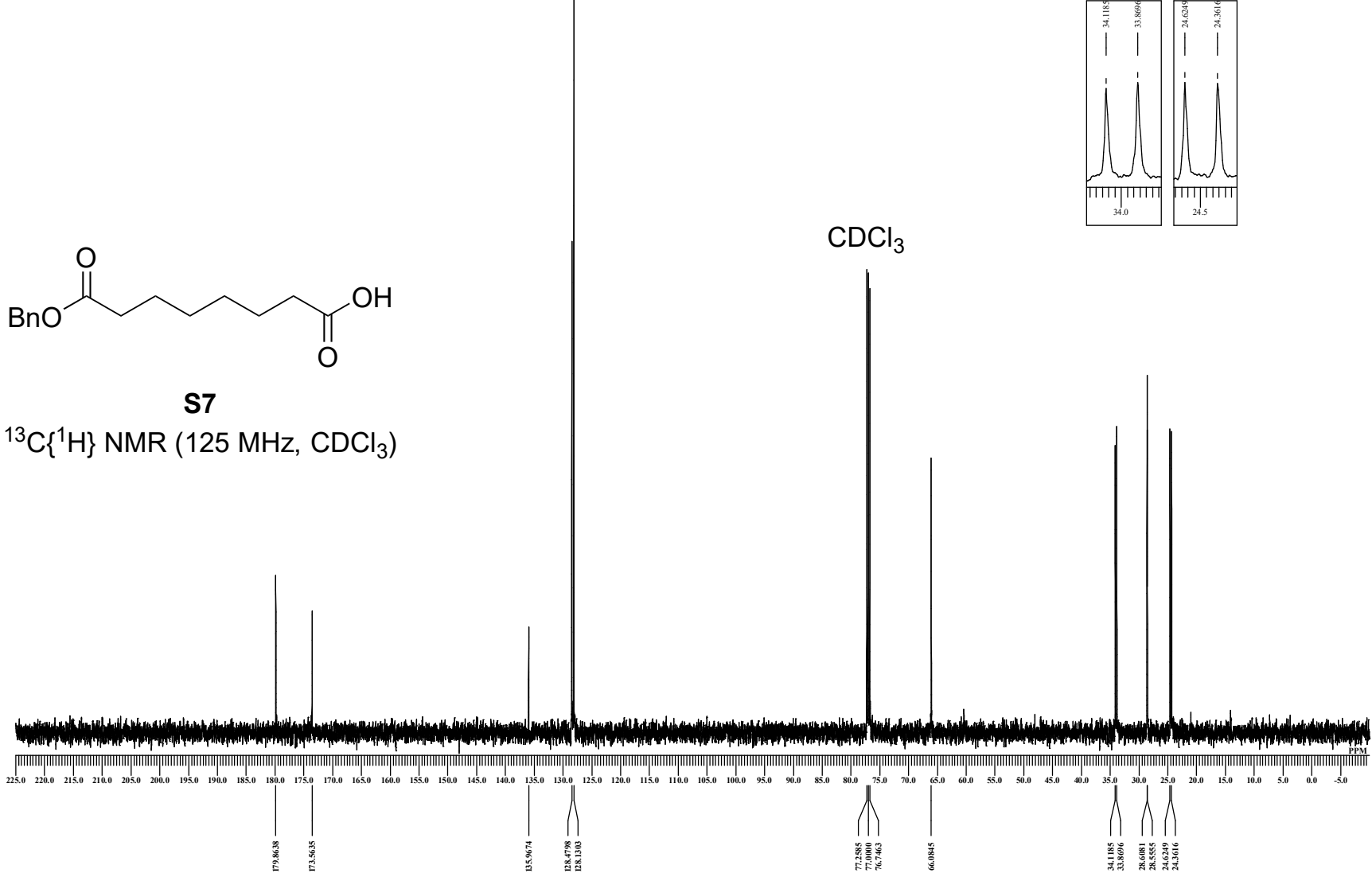


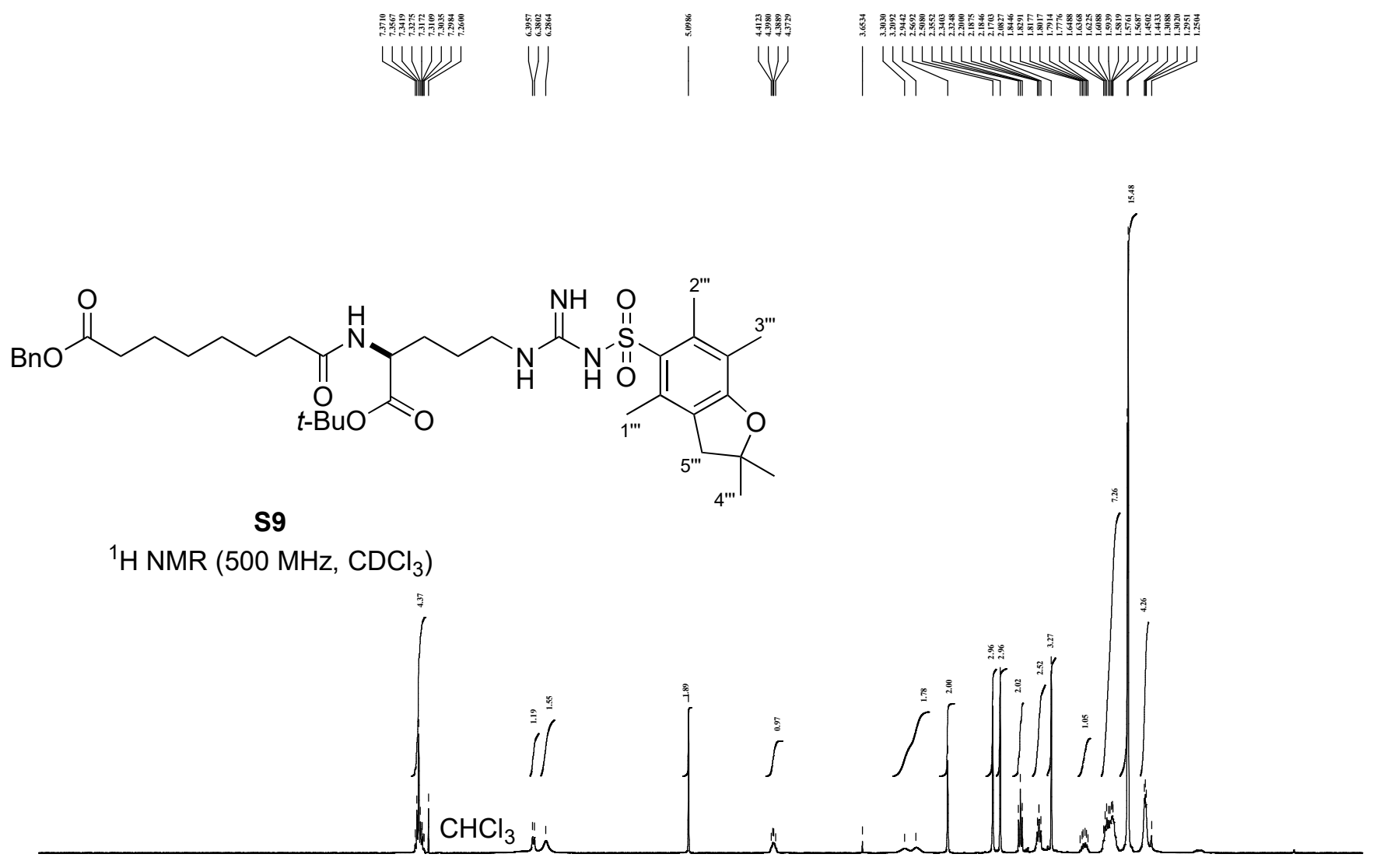

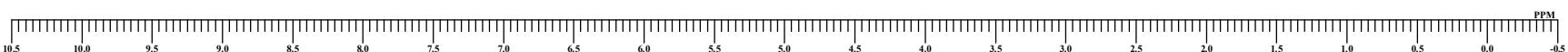

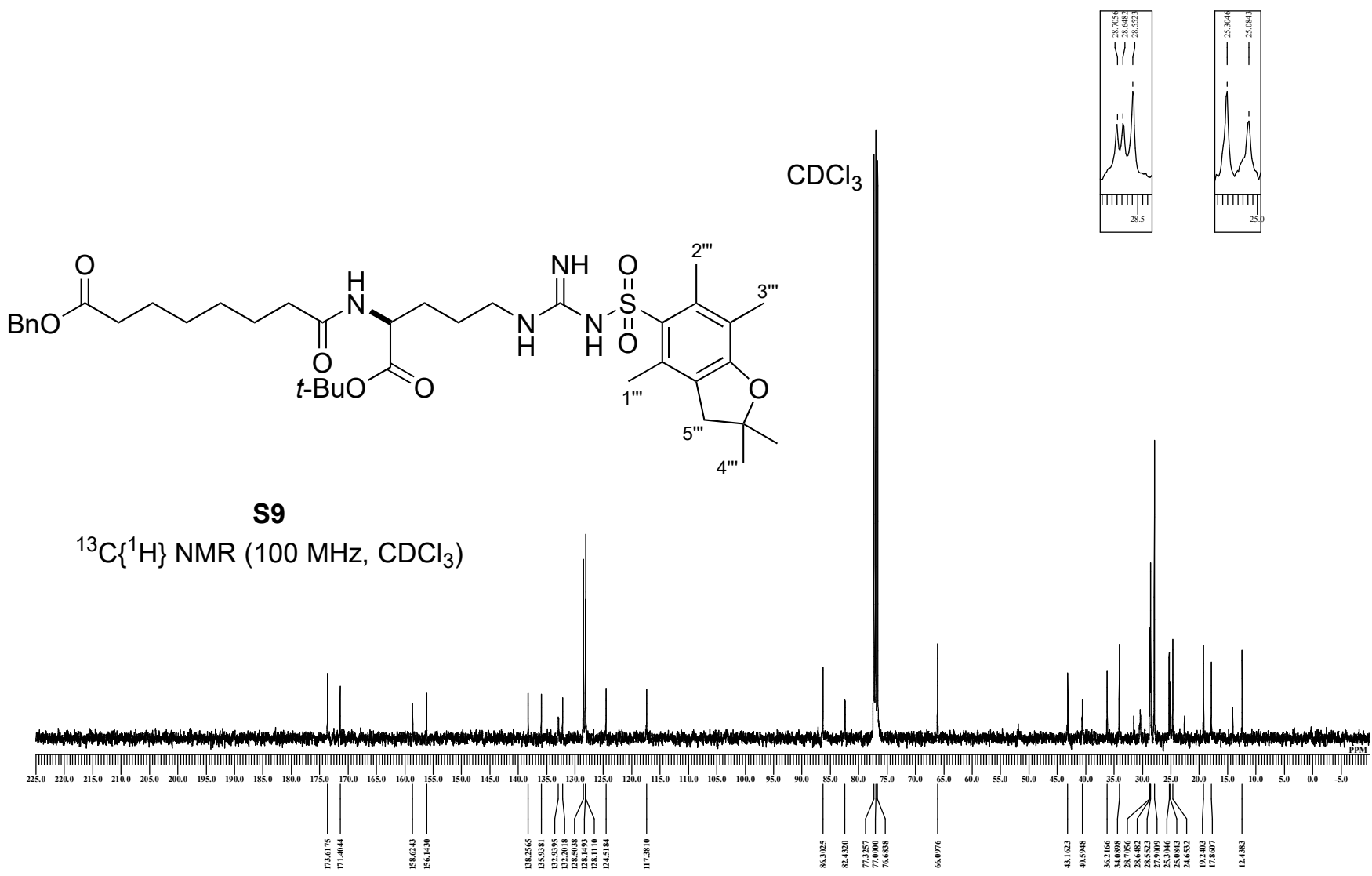




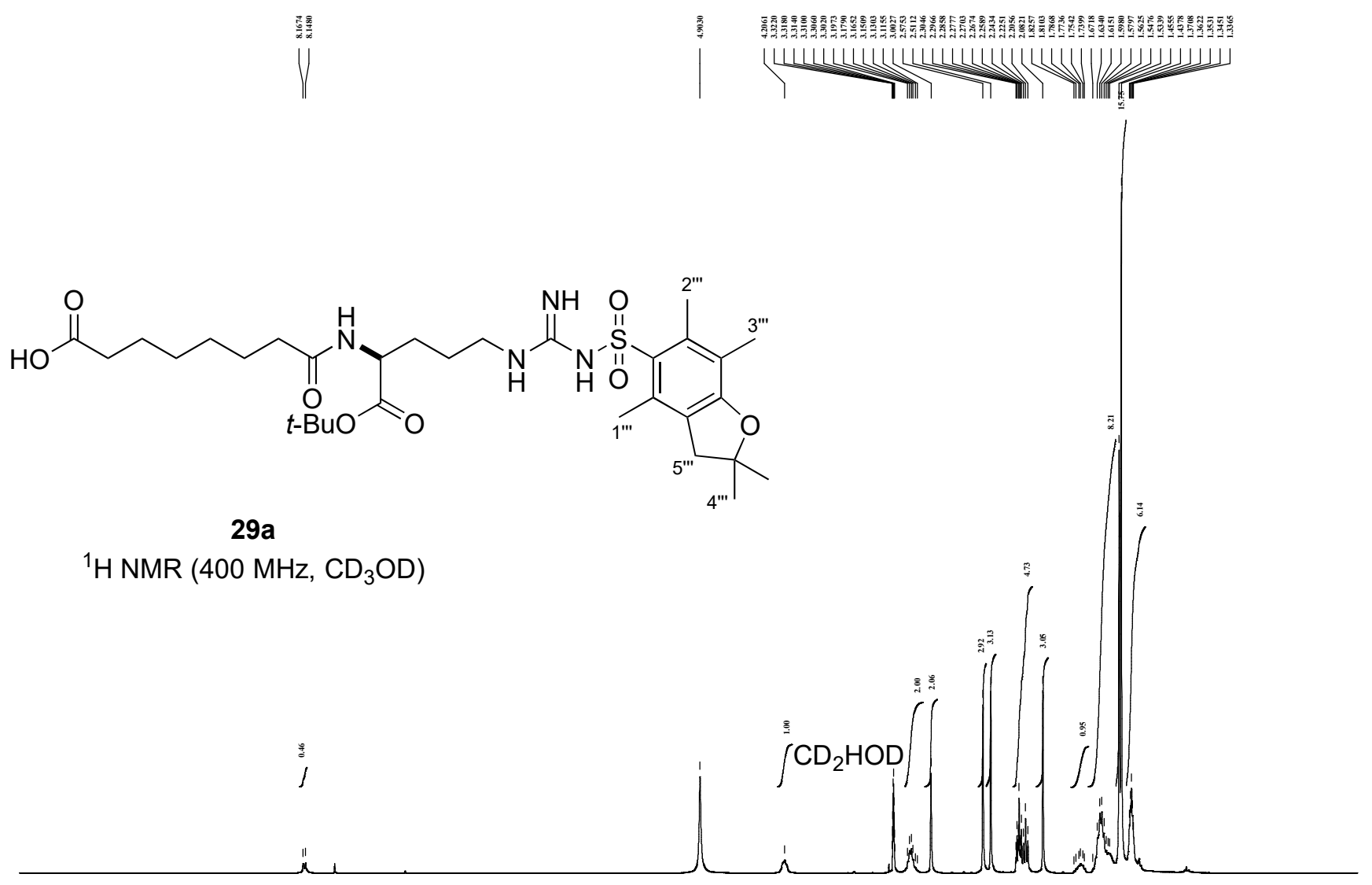

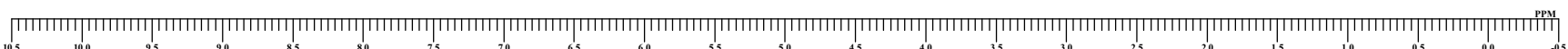

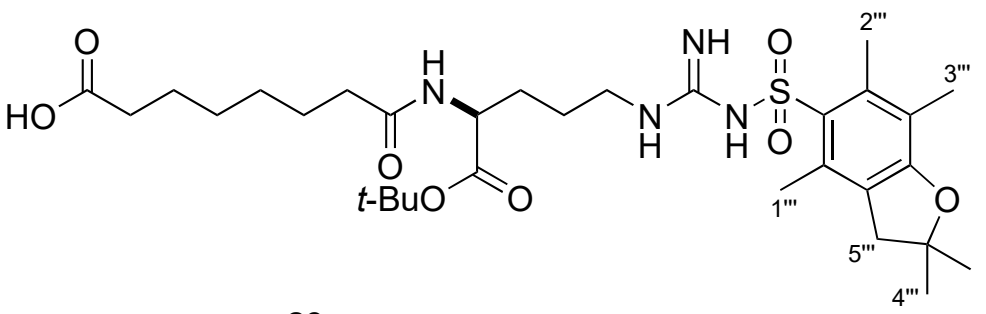

29a

${ }^{13} \mathrm{C}\left\{{ }^{1} \mathrm{H}\right\}$ NMR $\left(125 \mathrm{MHz}, \mathrm{CD}_{3} \mathrm{OD}\right)$

m | $\left.||\right|_{0}$ 


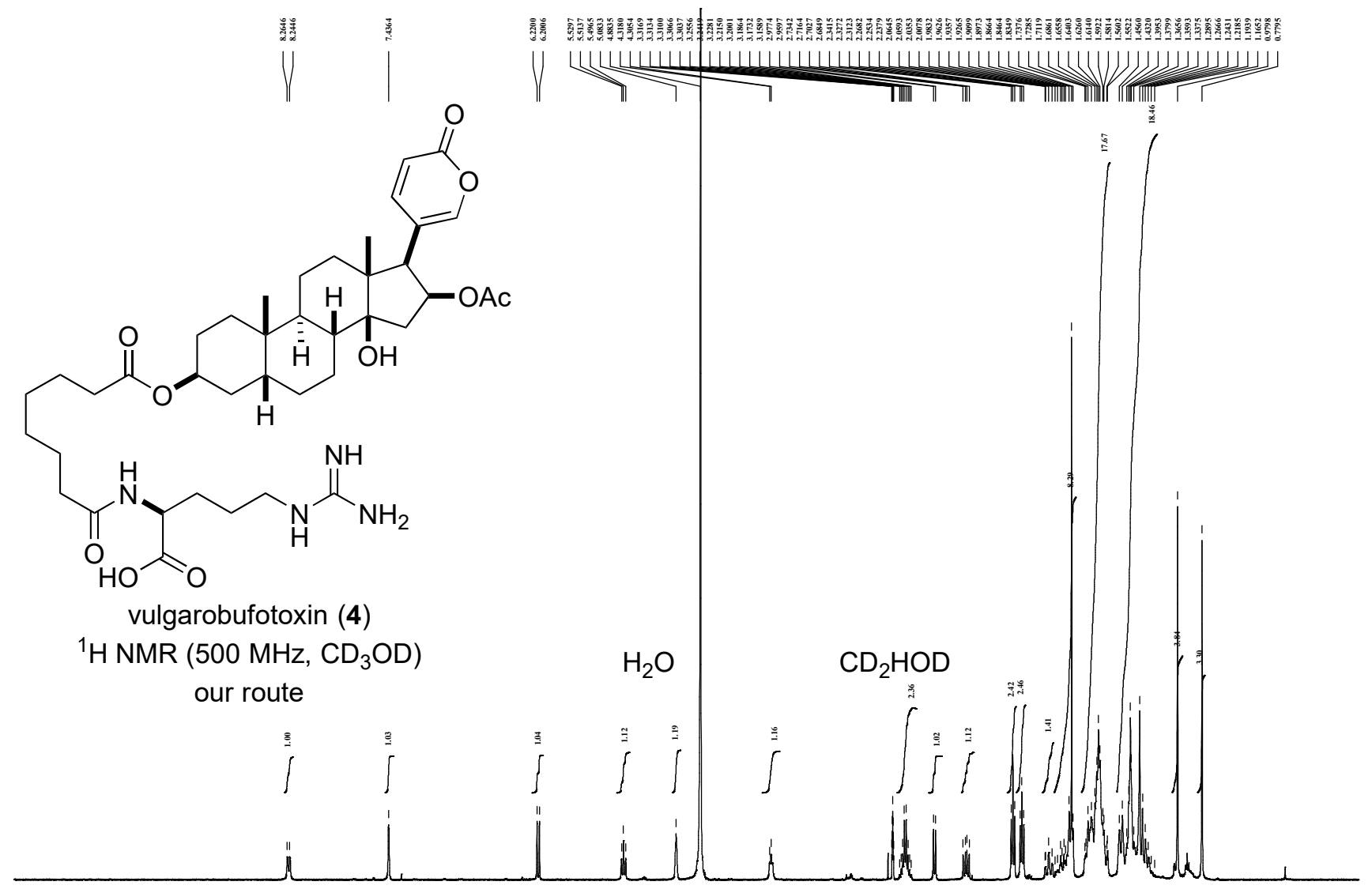

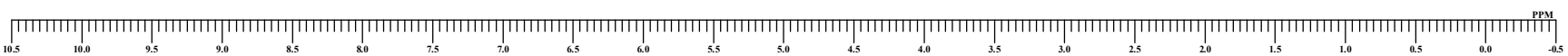

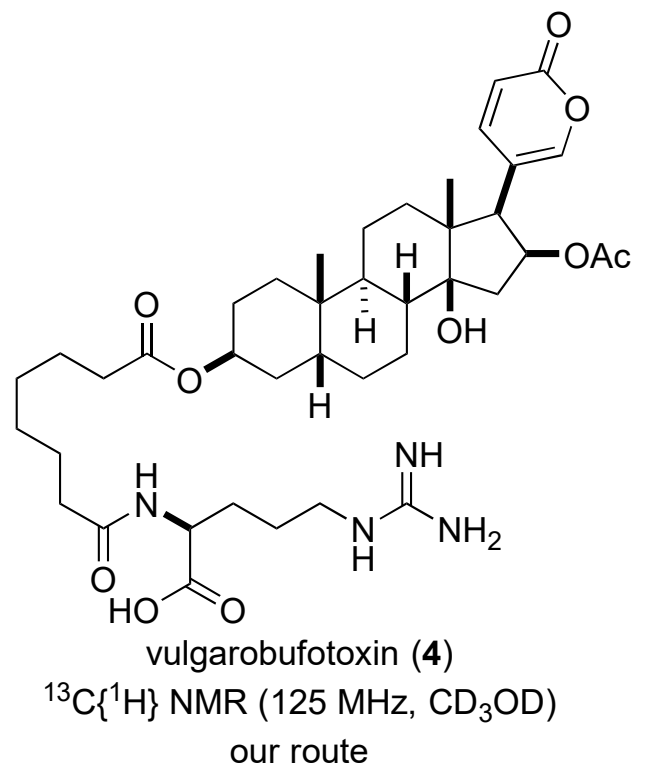

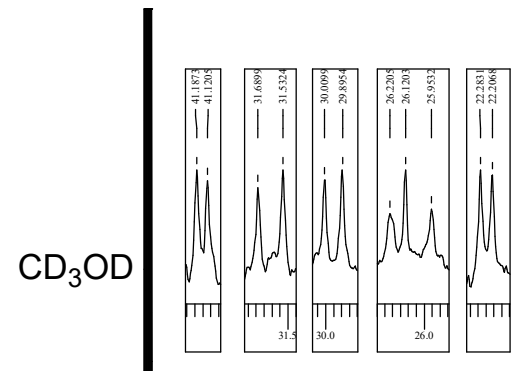

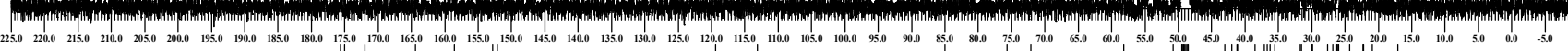




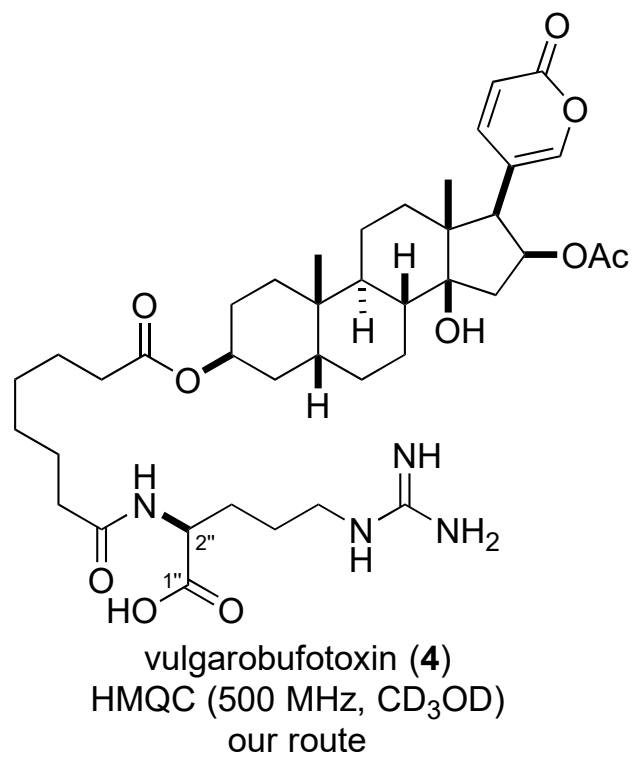

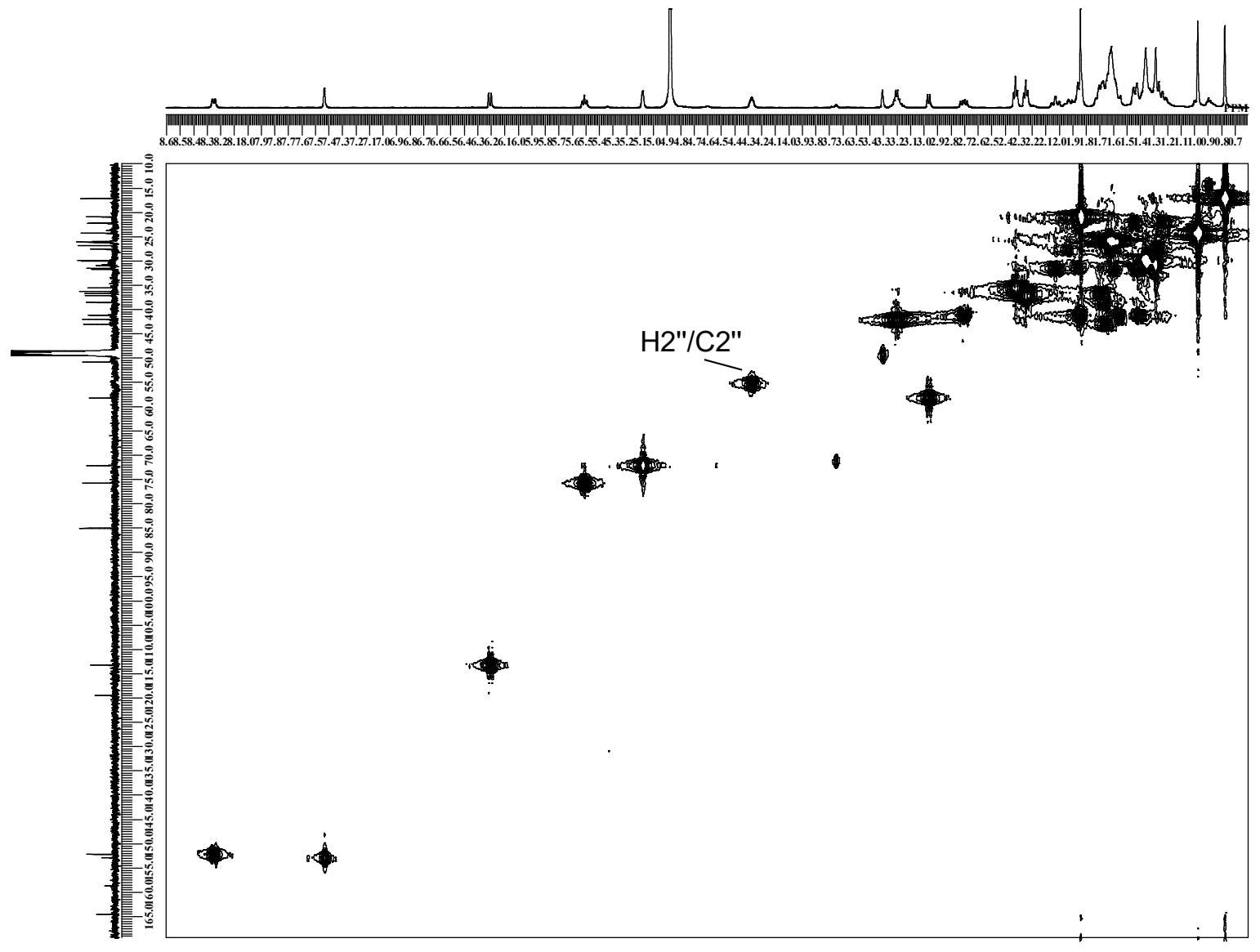


$\mathrm{HMBC}\left(500 \mathrm{MHz}, \mathrm{CD}_{3} \mathrm{OD}\right)$
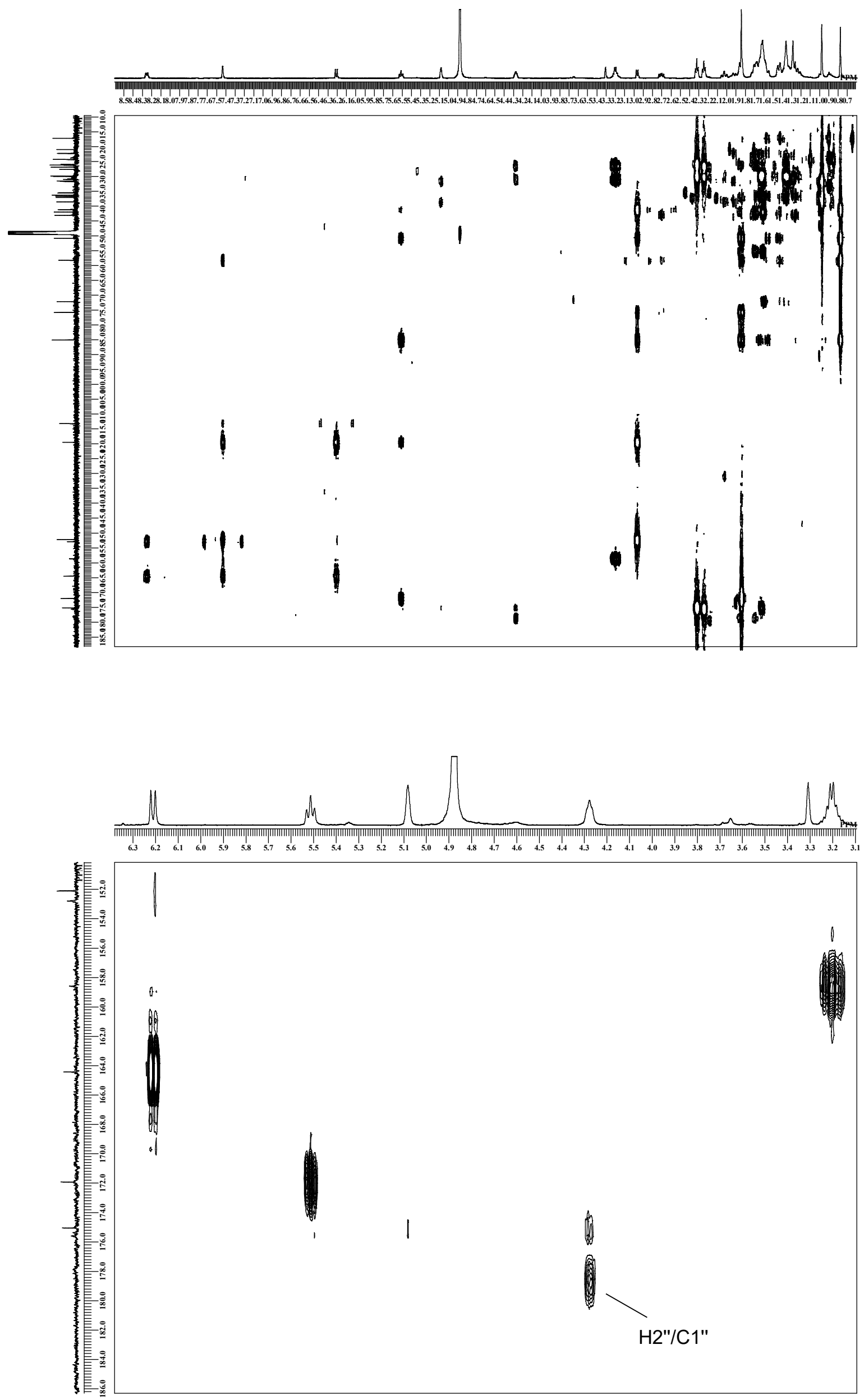

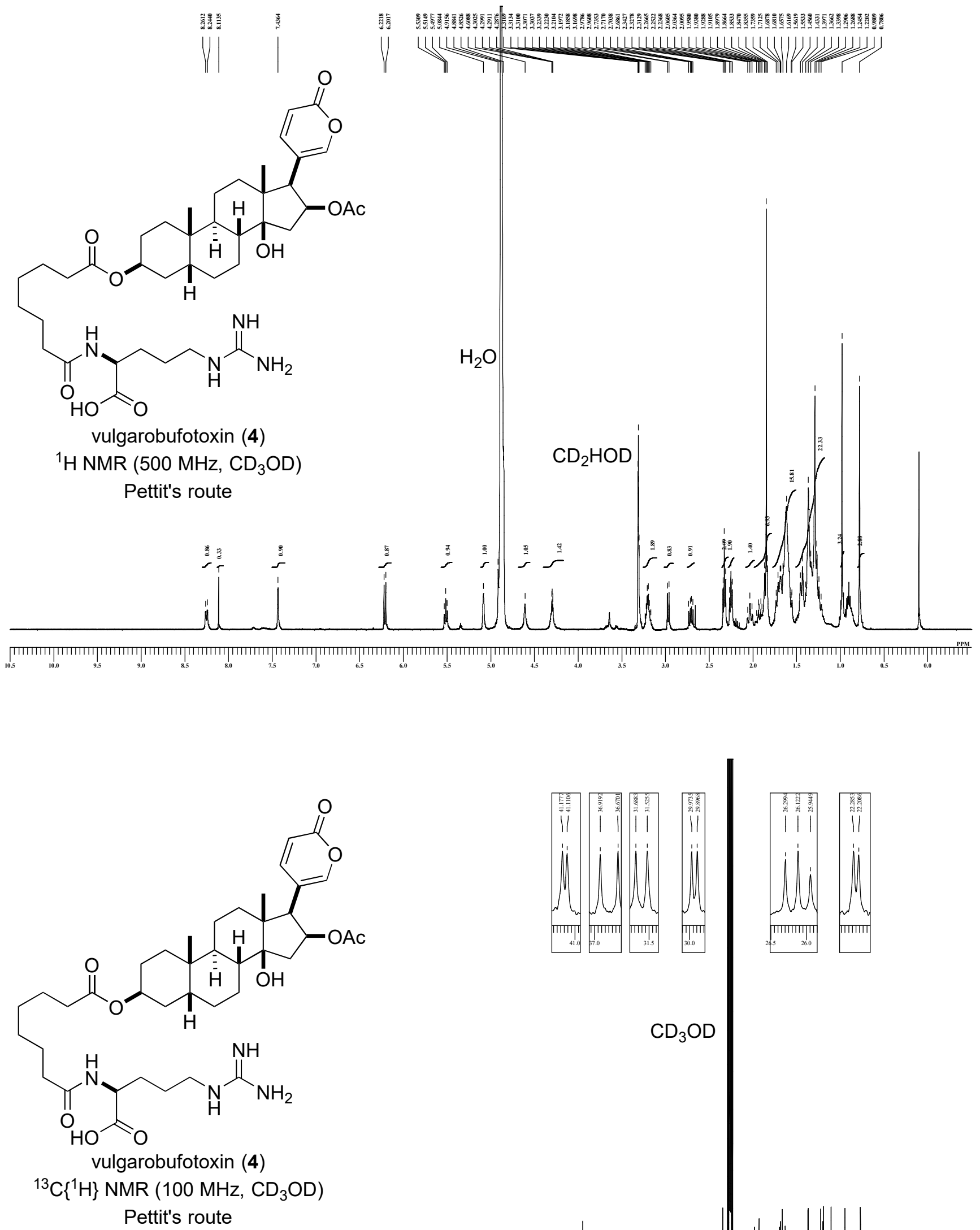

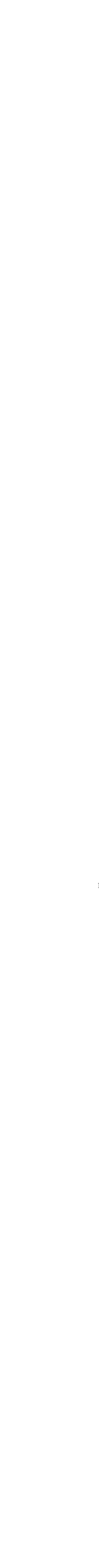

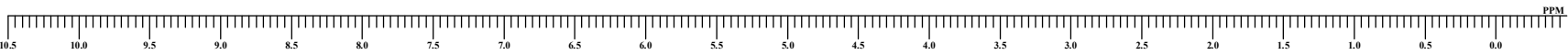

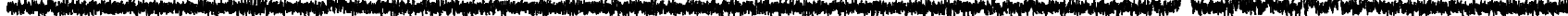
m

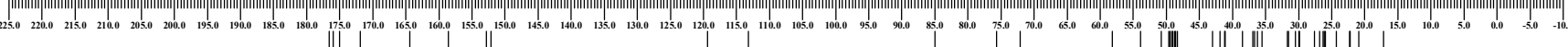

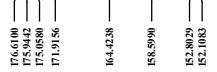



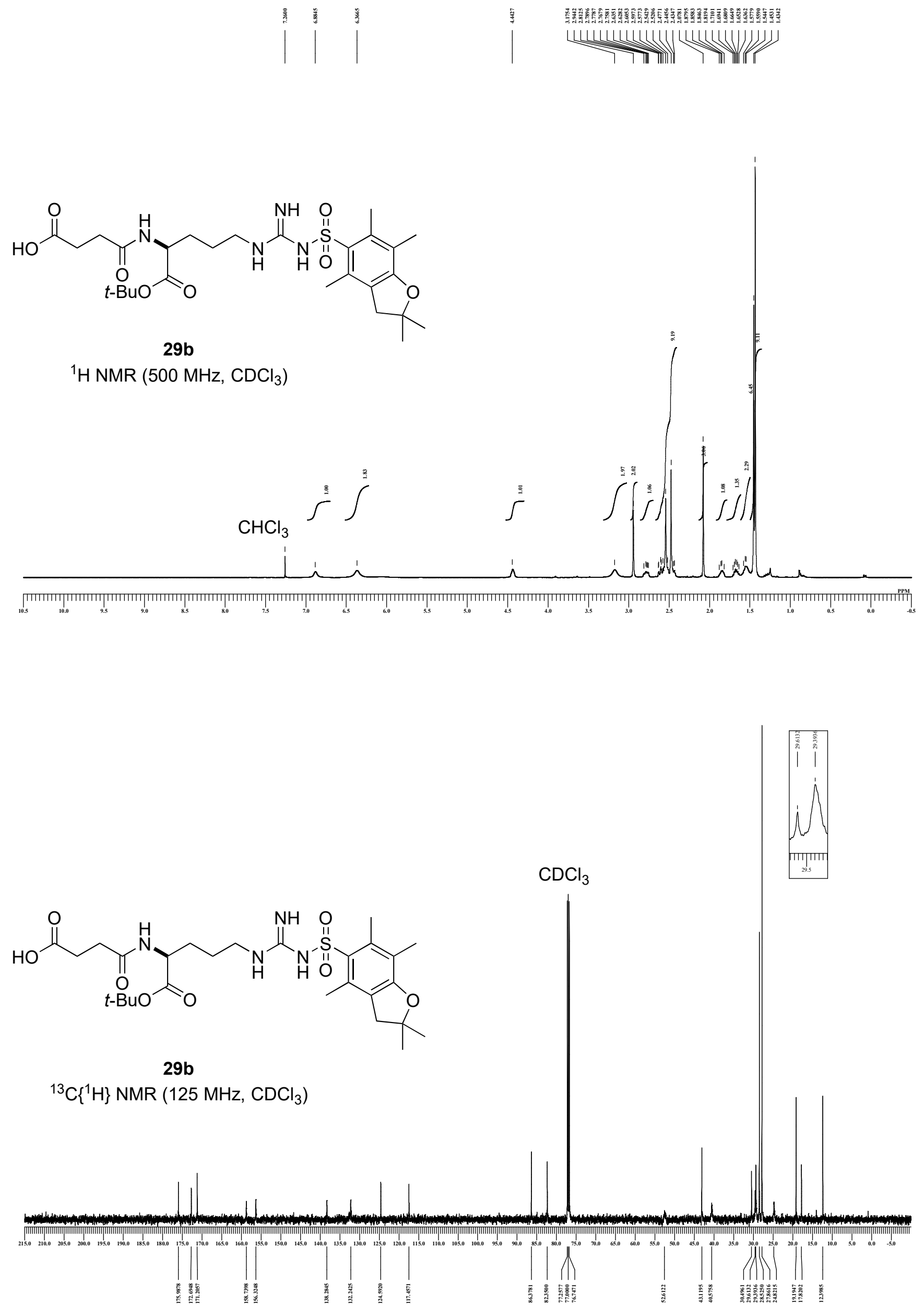

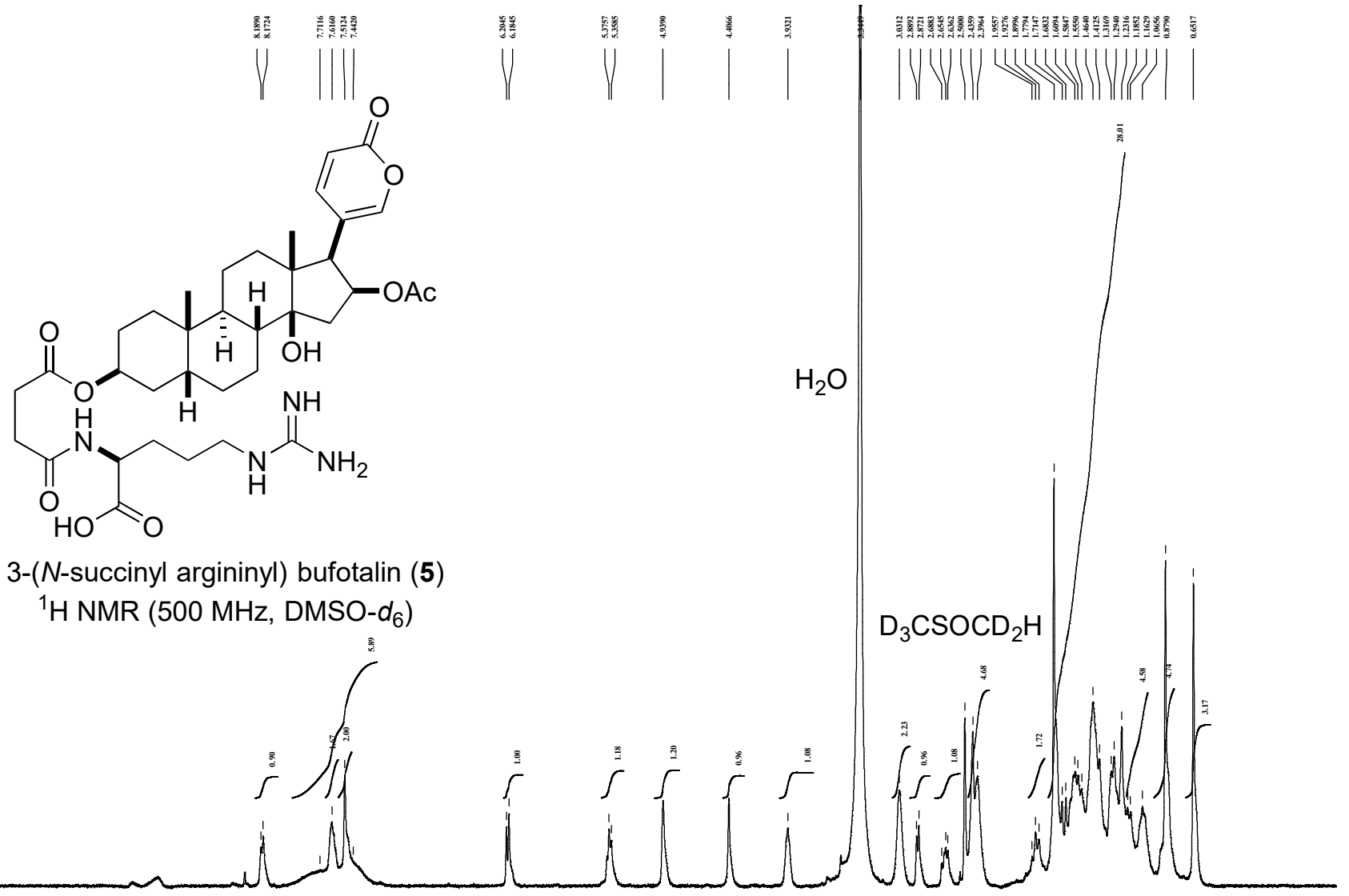

3-(N-succinyl argininyl) bufotalin (5)

${ }^{1} \mathrm{H}$ NMR $\left(500 \mathrm{MHz}\right.$, DMSO- $\left.d_{6}\right)$

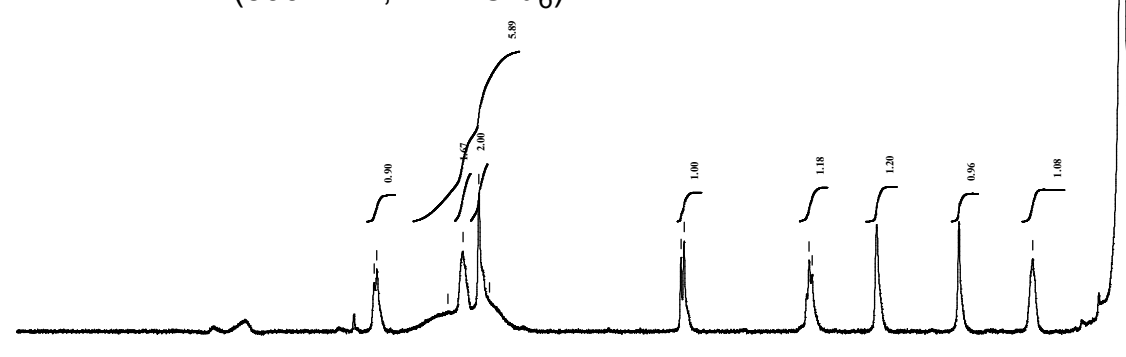

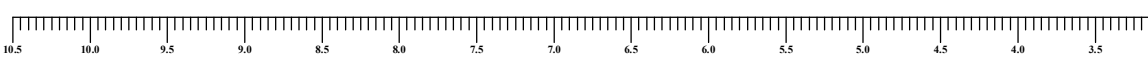
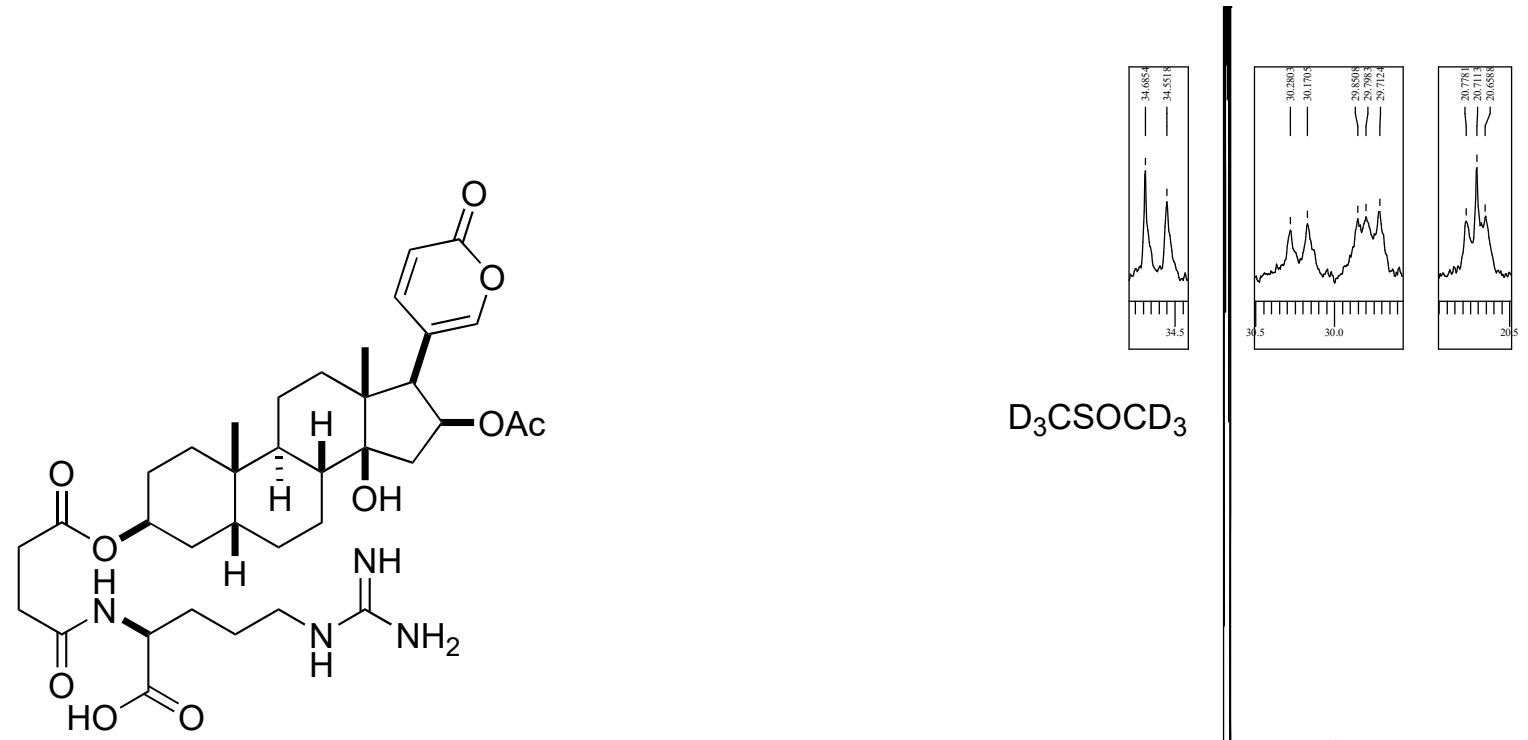

3-(N-succinyl argininyl) bufotalin (5)

${ }^{13} \mathrm{C}\left\{{ }^{1} \mathrm{H}\right\}$ NMR $\left(125 \mathrm{MHz}\right.$, DMSO- $\left.d_{6}\right)$

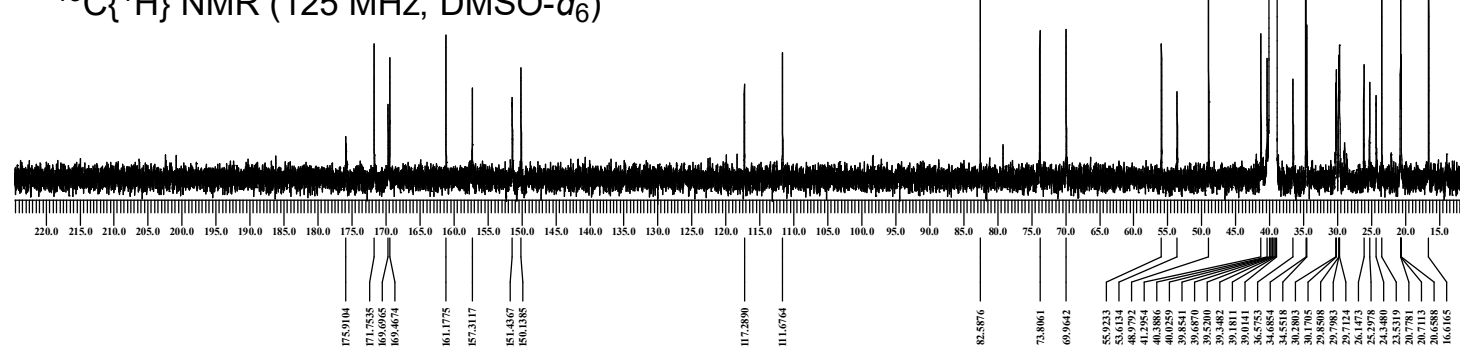

